\title{
The Star Formation in Radio Survey: 3-33 GHz Imaging of Nearby Galaxy Nuclei and Extranuclear Star-forming Regions
}

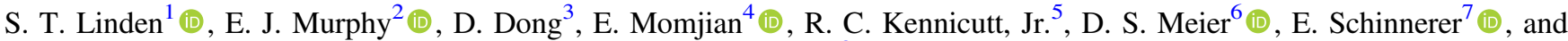 \\ J. L. Turner ${ }^{8}$ (i) \\ ${ }^{1}$ Department of Astronomy, University of Virginia, 530 McCormick Road, Charlottesville, VA 22904, USA \\ ${ }^{2}$ National Radio Astronomy Observatory, 520 Edgemont Road, Charlottesville, VA 22903, USA \\ ${ }^{3}$ California Institute of Technology, MC 100-22, Pasadena, CA 91125, USA \\ ${ }^{4}$ National Radio Astronomy Observatory, P.O. Box O, 1003 Lopezville Road, Socorro, NM 87801, USA \\ ${ }^{5}$ Institute of Astronomy, University of Cambridge, Madingley Road, Cambridge CB3 OHA, UK \\ ${ }^{6}$ New Mexico Institute of Mining and Technology, 801 Leroy Place, Socorro, NM 87801, USA \\ ${ }^{7}$ Max Planck Institut für Astronomie, Königstuhl 17, Heidelberg D-69117, Germany \\ ${ }^{8}$ Department of Physics and Astronomy, UCLA, Los Angeles, CA 90095, USA \\ Received 2020 February 10; revised 2020 March 20; accepted 2020 April 15; published 2020 May 28
}

\begin{abstract}
We present 3,15, and $33 \mathrm{GHz}$ imaging toward galaxy nuclei and extranuclear star-forming regions using the Karl G. Jansky Very Large Array as part of the Star Formation in Radio Survey. With 3-33 GHz radio spectra, we measured the spectral indices and corresponding thermal (free-free) emission fractions for a sample of 335 discrete regions having significant detections in at least two radio bands. After removing 14 likely background galaxies, we find that the median thermal fraction at $33 \mathrm{GHz}$ is $92 \% \pm 0.8 \%$ with a median absolute deviation of $11 \%$, when a two-component power-law model is adopted to fit the radio spectrum. Limiting the sample to 238 sources that are confidently identified as star-forming regions and not affected by potential AGN contamination (i.e., having galactocentric radii $r_{\mathrm{G}} \geqslant 250 \mathrm{pc}$ ) results in a median thermal fraction of $93 \% \pm 0.8 \%$ with a median absolute deviation of $10 \%$. We further measure the thermal fraction at $33 \mathrm{GHz}$ for 163 regions identified at 7 " resolution to be $94 \% \pm 0.8 \%$ with a median absolute deviation of $8 \%$. Together, these results confirm that free-free emission dominates the radio spectra of star-forming regions on scales up to $\sim 500 \mathrm{pc}$ in normal star-forming galaxies. We additionally find a factor of $\sim 1.6$ increase in the scatter of the measured spectral index and thermal fraction distributions as a function of decreasing galactocentric radius. This trend is likely reflective of the continuous star formation activity occurring in the galaxy centers, resulting in a larger contribution of diffuse nonthermal emission relative to star-forming regions in the disk.
\end{abstract}

Unified Astronomy Thesaurus concepts: Star formation (1569); H II regions (694); Radio continuum emission (1340); Star forming regions (1565); Radio interferometry (1346); Radio astronomy (1338); Galaxies (573); Galaxy evolution (594); Spiral galaxies (1560); Extragalactic radio sources (508)

\section{Introduction}

The radio spectra of star-forming galaxies, typically characterized as a power law $\left(S_{\nu} \propto \nu^{\alpha}\right)$, encode information about the thermal and nonthermal energetic processes that power them. Both thermal (bremsstrahlung) emission and nonthermal (synchrotron) emission are associated with massive $\left(\geqslant 8 M_{\odot}\right)$ star formation, underlying the basis for the wellknown correlation between far-infrared (FIR: $42-122 \mu \mathrm{m}$ ) and radio (de Jong et al. 1985; Helou et al. 1985; Condon 1992; Bell 2003). FIR emission arises from the absorption and reradiation of UV and optical photons that heat dust grains surrounding massive star-forming regions. The $\mathrm{O}$ and $\mathrm{B}$ stars in such regions, with lifetimes of $\leqslant 10 \mathrm{Myr}$, produce ionizing (Lyman continuum) radiation whose strength is directly proportional to the amount of free-free emission. These same massive stars end their lives as core-collapse supernovae, whose remnants accelerate cosmic-ray (CR) electrons/positrons that produce the diffuse nonthermal synchrotron emission observed in star-forming galaxies (Condon 1992; Koyama et al. 1995; Murphy et al. 2006; Lacki \& Thompson 2010; Lacki et al. 2010).

However, the connection between the nonthermal synchrotron emission and the current star formation rate (SFR) of a galaxy is far less direct relative to thermal free-free emission.
Variations in the generation and propagation of CRs through the interstellar medium (ISM) can affect the observed lowfrequency emission surrounding star-forming regions. Despite the complexity in interpreting this nonthermal emission from galaxies, several empirical (Koyama et al. 1995; Bell 2003; Murphy et al. 2006; Heesen et al. 2014; Tabatabaei et al. 2017) and theoretical (Condon 1992; Murphy et al. 2011) calibrations for the SFR exist in the literature. These studies demonstrate that at frequencies low enough (typically $\sim 1 \mathrm{GHz}$ ) the emission is dominated by the nonthermal, steep-spectrum component $\left(\alpha^{\mathrm{NT}} \sim-0.8\right)$, and at frequencies high enough $(\sim 30 \mathrm{GHz})$ the emission becomes dominated by thermal emission $\left(\alpha^{\mathrm{T}} \sim-0.1\right)$. Hence, radio observations can serve as an excellent, extinction-free diagnostic for the current SFR within nearby galaxies.

This was the motivation for initiating the Star Formation in Radio Survey (SFRS), which began as a $33 \mathrm{GHz}$ imaging campaign with the Green Bank Telescope (GBT) to study 103 galaxy nuclei and extranuclear star-forming complexes at a matched resolution of $25^{\prime \prime}$. In the initial investigation, Murphy et al. (2012) used the Westerbork Synthesis Radio Telescope (WSRT) in combination with the GBT to construct $1.7-33 \mathrm{GHz}$ radio spectra for 53 galaxy nuclei and extranuclear star-forming regions on $\sim$ kiloparsec scales. They found evidence that the measured thermal fraction at $33 \mathrm{GHz}$ varied significantly for 
star-forming regions observed at different physical resolution due to the range in galaxy distance. Photometric apertures larger than $\sim 1 \mathrm{kpc}$ were observed to have thermal fractions as low as $40 \%-50 \%$, whereas regions measured with apertures $\leqslant 1 \mathrm{kpc}$ appeared to be heavily dominated by free-free emission, with thermal fractions as high as $\sim 90 \%$. However, without high-resolution maps of both free-free and nonthermal emission from individual $\mathrm{H}$ II regions within these larger complexes, it is difficult to determine the physical nature of these trends within nearby galaxies.

This study served as the foundation for extending the SFRS into a multifrequency Karl G. Jansky Very Large Array (VLA) campaign to image hundreds of star-forming regions in 50 galaxies taken from the Spitzer Infrared Nearby Galaxies Survey (SINGS; Kennicutt et al. 2003) and the Key Insights on Nearby Galaxies: a Far-Infrared Survey (KINGFISH; Kennicutt et al. 2011). The results from our $33 \mathrm{GHz}$ observations, along with corresponding $\mathrm{H} \alpha$ and Spitzer/MIPS $24 \mu \mathrm{m}$ photometry, were recently presented in Murphy et al. (2018a, hereafter M18a) and explored the $\mathrm{H} \alpha$-to-33 GHz and $24 \mu \mathrm{m}$-to$33 \mathrm{GHz}$ flux density ratios of star-forming regions as a function of galactocentric radius and physical resolution. An outlier of these distributions, NGC $4725 \mathrm{~B}$, was later followed up with higher-frequency observations ( $Q$ band: $\sim 44 \mathrm{GHz}$ ) in order to confirm this region as the second known source of extragalactic anomalous microwave emission (AME; Murphy et al. 2018b). Building on this analysis, we have also obtained 3 and $15 \mathrm{GHz}$ imaging for the SFRS, allowing us to map the full radio spectrum of each star-forming region at a matched resolution of $\sim 2^{\prime \prime}$. In this paper, we focus our presentation on the results associated with the radio spectral indices and corresponding free-free emission fractions for the entire sample.

The paper is organized as follows: In Section 2 we describe our sample selection, data reduction, and imaging procedure for the 3-33 GHz VLA data. In Section 3 we describe the ancillary data products included in this study, as well as the analysis procedures used. Our results are presented in Section 4 and discussed in Section 5. Our main conclusions are summarized in Section 6. In the Appendix we additionally provide ancillary photometry from the Galaxy Evolution Explorer (GALEX), Spitzer, and ground-based $\mathrm{H} \alpha$ observations at a matched resolution of $7^{\prime \prime}$ that are not used in the present analysis. Throughout the paper we report the median absolute deviations rather than standard deviations, as this statistic is more resilient against outliers in a data set.

\section{Sample and Data Analysis}

In this section we describe the sample selection and present the VLA observations along with the data reduction and imaging procedures.

\subsection{Sample Selection}

The SFRS sample includes targeted observations from 56 nearby galaxies $\left(d_{L}<30 \mathrm{Mpc}\right)$ in the SINGS and KINGFISH legacy programs (Table 1). All nuclear and extranuclear starforming regions were chosen to have mid-infrared spectral mappings carried out by the IRS instrument on board Spitzer and Herschel/PACS FIR spectral mappings, for a combination of the principal atomic ISM cooling lines ([O I] $63 \mu \mathrm{m}$, [O III] $88 \mu \mathrm{m},[\mathrm{N}$ II] 122, $205 \mu \mathrm{m}$, and [C II] $158 \mu \mathrm{m})$. NGC 5194 and
NGC 2403 are exceptions; these galaxies were part of the SINGS sample but are not formally included in KINGFISH. They were observed with Herschel as part of the Very Nearby Galaxy Survey (VNGS; PI: C. Wilson). Similarly, there are additional KINGFISH galaxies that were not part of SINGS but have existing Spitzer data: NGC 5457 (M101), IC 342, NGC 3077, and NGC 2146.

The SINGS and KINGFISH galaxies are fully representative of the integrated properties and ISM conditions found in the local universe, spanning the full range in morphological types, as well as a factor of 100 in IR luminosity $\left(L_{\mathrm{IR}}: 8-1000 \mu \mathrm{m}\right)$, global IR/optical flux ratio, and the SFR. Similarly, the spectroscopically targeted star-forming regions included in the SFRS cover the full range of physical conditions found in nearby galaxies, including the extinction-corrected production rate of ionizing photons $Q\left(H_{0}\right)$, metallicity, visual extinction, radiation field intensity, and ionizing stellar temperature.

The full sample over the entire sky consists of 118 star-forming complexes (56 nuclei and 62 extranuclear regions), 112 of which (50 nuclei and 62 extranuclear regions; see Tables 2 and 3, respectively) are observable with the VLA (i.e., having $\delta>-35^{\circ}$ ). The coordinates given in both tables list the pointing center for the VLA observations (see Section 2.2), which correspond to the centers of the Spitzer mid-infrared and Herschel FIR spectral line maps. Morphologies, adopted distances, optically defined nuclear types, diameters $\left(D_{25}\right)$, inclinations $(i)$, and position angles (P.A.) for each source are given in Table 1 and described in detail in M18a.

\subsection{VLA Observations and Data Reduction}

The observational setup and reduction procedure for the $\mathrm{Ka}$ band (29-37 GHz) data (11B-032,13A-129) is described in detail in M18a. Observations in the $S$ band (2-4 GHz) were taken during the 2013 VLA B-configuration cycle (13B-215) and utilized the 8-bit sampler. Observations in the $\mathrm{Ku}$ band $(12-18 \mathrm{GHz})$ were taken in 2014 November in the $\mathrm{C}$ configuration (13B-215) using the 3-bit samplers. Both sets of observations utilized the full available bandwidth of the respective receivers. Given the large range in brightness among our targeted regions, we varied the time spent on source by estimating the expected $3-15 \mathrm{GHz}$ flux density using the Spitzer/MIPS $24 \mu \mathrm{m}$ maps. The median integration time for regions in our sample was $\sim 10$ minutes at both frequencies. The choice of array configurations was made to match the angular resolution (i.e., FWHM of the synthesized beam $\sim 2^{\prime \prime}$ ) of the observations at each band. This allows us to probe the same spatial scales across the full $3-33 \mathrm{GHz}$ frequency range and ensures that any differences in the measured spectral index of individual star-forming regions are due to physical variation in the region being measured, and not due to resolving out more emission at higher frequencies.

The standard VLA flux density calibrators 3C 48, 3C 286, and 3C 147 were used, and the data reduction procedures presented in M18a are repeated for our present analysis and briefly described here. To reduce the VLA data, we used the Common Astronomy Software Applications (CASA; McMullin et al. 2007) versions 4.6.0 and 4.7.0 and followed standard calibration and flagging procedures, including the utilization of the VLA calibration pipeline. We further inspected the visibilities and calibration tables for evidence of bad antennas, frequency ranges, and time ranges, flagging correspondingly. We also flagged any instances of radio frequency interference 
Table 1

Galaxy Properties

\begin{tabular}{|c|c|c|c|c|c|c|}
\hline Galaxy & Type $^{\mathrm{a}}$ & $\begin{array}{l}\text { Dist. }^{\mathrm{b}} \\
\text { (Mpc) }\end{array}$ & Nuc. Type & $\begin{array}{c}D_{25}{ }^{\mathrm{a}} \\
(\operatorname{arcmin})\end{array}$ & $\begin{array}{c}i \\
(\mathrm{deg})\end{array}$ & $\begin{array}{l}\text { P.A. }^{a} \\
(\text { deg })\end{array}$ \\
\hline NGC 0337 & SBd & 19.3 & SF & $2.9 \times 1.8$ & 52 & 130 \\
\hline NGC 0628 & SAc & 7.2 & $\cdots$ & $10.5 \times 9.5$ & 25 & 25 \\
\hline NGC 0855 & $\mathrm{E}$ & 9.73 & SF & $2.6 \times 1.0$ & 70 & $67^{\mathrm{d}}$ \\
\hline NGC 0925 & SABd & 9.12 & SF & $10.5 \times 5.9$ & 57 & 102 \\
\hline NGC 1097 & $\mathrm{SBb}$ & 14.2 & AGN & $9.3 \times 6.3$ & 48 & 130 \\
\hline NGC 1266 & SB0 & 30.6 & $\mathrm{AGN}$ & $1.5 \times 1.0$ & 49 & $108^{d}$ \\
\hline NGC 1377 & S0 & 24.6 & $\ldots$ & $1.8 \times 0.9$ & 61 & 92 \\
\hline IC 0342 & SABcd & 3.28 & $\mathrm{SF}\left({ }^{*}\right)$ & $21.4 \times 20.0$ & 21 & $153^{\mathrm{d}}$ \\
\hline NGC 1482 & SA0 & 22.6 & SF & $2.5 \times 1.4$ & 57 & 103 \\
\hline NGC 2146 & Sbab & 17.2 & $\mathrm{SF}\left(^{*}\right)$ & $6.0 \times 3.4$ & 56 & 57 \\
\hline NGC 2403 & SABcd & 3.22 & $\mathrm{SF}\left({ }^{*}\right)$ & $21.9 \times 12.3$ & 57 & 128 \\
\hline Holmberg II & $\mathrm{Im}$ & 3.05 & $\cdots$ & $7.9 \times 6.3$ & 37 & 16 \\
\hline NGC 2798 & $\mathrm{SBa}$ & 25.8 & $\mathrm{SF} / \mathrm{AGN}$ & $2.6 \times 1.0$ & 70 & 160 \\
\hline NGC 2841 & $\mathrm{SAb}$ & 14.1 & $\mathrm{AGN}$ & $8.1 \times 3.5$ & 66 & 147 \\
\hline NGC 2976 & SAc & 3.55 & SF & $5.9 \times 2.7$ & 64 & 143 \\
\hline NGC 3049 & SBab & 19.2 & SF & $2.2 \times 1.4$ & 51 & 25 \\
\hline NGC 3077 & I0pec & 3.83 & $\mathrm{SF}\left(^{*}\right)$ & $5.4 \times 4.5$ & 34 & 45 \\
\hline NGC 3190 & SAap & 19.3 & $\mathrm{AGN}\left({ }^{*}\right)$ & $4.4 \times 1.5$ & 73 & 125 \\
\hline NGC 3184 & SABcd & 11.7 & SF & $7.4 \times 6.9$ & 21 & 135 \\
\hline NGC 3198 & $\mathrm{SBc}$ & 14.1 & SF & $8.5 \times 3.3$ & 68 & 35 \\
\hline IC 2574 & $\mathrm{SABm}$ & 3.79 & $\mathrm{SF}\left(^{*}\right)$ & $13.2 \times 5.4$ & 67 & 50 \\
\hline NGC 3265 & $\mathrm{E}$ & 19.6 & $\mathrm{SF}$ & $1.3 \times 1.0$ & 39 & 73 \\
\hline NGC 3351 & $\mathrm{SBb}$ & 9.33 & SF & $7.4 \times 5.0$ & 48 & 13 \\
\hline NGC 3521 & $\mathrm{SABbc}$ & 11.2 & $\mathrm{SF} / \mathrm{AGN}\left(^{*}\right)$ & $11.0 \times 5.1$ & 63 & 163 \\
\hline NGC 3621 & SAd & 6.55 & AGN & $12.3 \times 7.1$ & 55 & 159 \\
\hline NGC 3627 & $\mathrm{SABb}$ & 9.38 & AGN & $9.1 \times 4.2$ & 64 & 173 \\
\hline NGC 3773 & SA0 & 12.4 & SF & $1.2 \times 1.0$ & 33 & 165 \\
\hline NGC 3938 & SAc & 17.9 & $\mathrm{SF}\left(^{*}\right)$ & $5.4 \times 4.9$ & 25 & $29^{\mathrm{d}}$ \\
\hline NGC 4254 & SAc & 14.4 & $\mathrm{SF} / \mathrm{AGN}$ & $5.4 \times 4.7$ & 30 & $24^{\mathrm{d}}$ \\
\hline NGC 4321 & SABbc & 14.3 & $\mathrm{AGN}$ & $7.4 \times 6.3$ & 32 & 30 \\
\hline NGC 4536 & SABbc & 14.5 & SF/AGN & $7.6 \times 3.2$ & 66 & 130 \\
\hline NGC 4559 & SABcd & 6.98 & SF & $10.7 \times 4.4$ & 67 & 150 \\
\hline NGC 4569 & SABab & 9.86 & AGN & $9.5 \times 4.4$ & 64 & 23 \\
\hline NGC 4579 & $\mathrm{SABb}$ & 16.4 & AGN & $5.9 \times 4.7$ & 37 & 95 \\
\hline NGC 4594 & SAa & 9.08 & AGN & $8.7 \times 3.5$ & 69 & 90 \\
\hline NGC 4625 & SABmp & 9.3 & SF & $2.2 \times 1.9$ & 31 & $28^{\mathrm{d}}$ \\
\hline NGC 4631 & SBd & 7.62 & $\mathrm{SF}\left(^{*}\right)$ & $15.5 \times 2.7$ & 83 & 86 \\
\hline NGC 4725 & SABab & 11.9 & $\mathrm{AGN}$ & $10.7 \times 7.6$ & 45 & 35 \\
\hline NGC 4736 & SAab & 4.66 & $\mathrm{AGN}\left({ }^{*}\right)$ & $11.2 \times 9.1$ & 35 & 105 \\
\hline NGC 4826 & SAab & 5.27 & AGN & $10.0 \times 5.4$ & 59 & 115 \\
\hline NGC 5055 & SAbc & 7.94 & AGN & $12.6 \times 7.2$ & 56 & 105 \\
\hline NGC 5194 & SABbcp & 7.62 & AGN & $11.2 \times 6.9$ & 53 & 163 \\
\hline NGC 5398 & SBdm & 7.66 & $\ldots$ & $2.8 \times 1.7$ & 53 & 172 \\
\hline NGC 5457 & SABcd & 6.7 & $\mathrm{SF}\left(^{*}\right)$ & $28.8 \times 26.0$ & 26 & $29^{\mathrm{d}}$ \\
\hline NGC 5474 & SAcd & 6.8 & $\mathrm{SF}\left(^{*}\right)$ & $4.8 \times 4.3$ & 27 & $98^{\mathrm{d}}$ \\
\hline NGC 5713 & SABbcp & 21.4 & SF & $2.8 \times 2.5$ & 27 & 10 \\
\hline NGC 5866 & So & 15.3 & AGN & $4.7 \times 1.9$ & 69 & 128 \\
\hline NGC 6946 & SABcd & 6.8 & $\mathrm{SF}$ & $11.5 \times 9.8$ & 32 & $53^{\mathrm{d}}$ \\
\hline NGC 7331 & $\mathrm{SAb}$ & 14.5 & AGN & $10.5 \times 3.7$ & 72 & 171 \\
\hline NGC 7793 & SAd & 3.91 & SF & $9.3 \times 6.3$ & 48 & 98 \\
\hline
\end{tabular}

Notes.

${ }^{a}$ Morphological types, diameters, and position angles were taken from the Third Reference Catalog of Bright Galaxies (RC3; de Vaucouleurs et al. 1991).

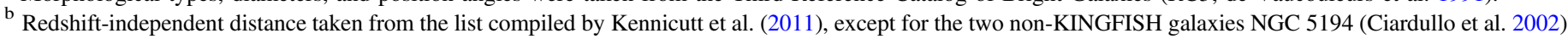
and NGC 2403 (Freedman et al. 2001).


Lonsdale Persson \& Helou (1987).

${ }^{\mathrm{d}}$ Position angle taken from Jarrett et al. (2003).

(RFI). Importantly, the fractional bandwidth of our observations lost to RFI flagging is negligible relative to the full bandwidth of the receivers. After flagging, we reran the pipeline, and we repeated this process until all poorly calibrated data were removed. For all delay and bandpass tables applied on the fly, we used the default nearest-neighbor interpolation. For complex gain and flux density scale tables, we used a linear interpolation. 
Table 2

Nuclear Source Positions and Imaging Characteristics

\begin{tabular}{|c|c|c|c|c|c|c|c|c|}
\hline \multirow[b]{2}{*}{ Galaxy } & \multirow[b]{2}{*}{$\begin{array}{l}\text { R.A. } \\
\text { (J2000) }\end{array}$} & \multirow[b]{2}{*}{$\begin{array}{l}\text { Decl. } \\
\text { (J2000) }\end{array}$} & \multicolumn{3}{|c|}{$3 \mathrm{GHz}$} & \multicolumn{3}{|c|}{$15 \mathrm{GHz}$} \\
\hline & & & $\begin{array}{l}\text { Synthesized } \\
\text { Beam }\end{array}$ & $\begin{array}{c}\sigma \\
\left(\mu \mathrm{Jy} \mathrm{bm}^{-1}\right)\end{array}$ & $\begin{array}{l}\sigma_{T_{\mathrm{b}}} \\
(\mathrm{mK})\end{array}$ & $\begin{array}{l}\text { Synthesized } \\
\text { Beam }\end{array}$ & $\begin{array}{c}\sigma \\
\left(\mu \mathrm{Jy} \mathrm{bm}^{-1}\right)\end{array}$ & $\begin{array}{c}\sigma_{T_{\mathrm{b}}} \\
(\mathrm{mK})\end{array}$ \\
\hline NGC 0337 & 005950.3 & -073444 & $2 ! " 43 \times 1 " .74$ & 18.7 & 597.32 & $2 ! \prime 00 \times 1 "$ "13 & 9.1 & 21.71 \\
\hline NGC 0628 & 013641.7 & +154659 & 1 ".96 × 1 !"78 & 14.0 & 543.15 & 1 " $55 \times 1$ " 19 & 9.6 & 28.14 \\
\hline NGC 0855 & 021403.7 & +275238 & 1 ". $80 \times 1$ × 62 & 13.2 & 613.61 & 1 ". $50 \times 1 " .25$ & 8.6 & 24.93 \\
\hline NGC 0925 & 022717.0 & +333443 & $1 " ! 80 \times 1 " .59$ & 13.0 & 611.30 & $1 " .47 \times 1 "$ × 23 & 9.1 & 27.04 \\
\hline NGC 1097 & 024619.1 & -301628 & $5 ! 76 \times 1 "$ " 80 & 44.9 & 586.45 & $3 ! " 81 \times 0 "$ " 99 & 16.9 & 24.11 \\
\hline NGC 1266 & 031600.8 & -022538 & $2 ! " 20 \times 1 ! " 78$ & 14.0 & 485.33 & $1 "$ " $80 \times 1 "$ " 17 & 11.3 & 28.86 \\
\hline NGC 1377 & 033638.9 & -205406 & 3 ". $56 \times 1$ ×. 71 & 17.4 & 385.40 & 2 !" $61 \times 1$ ". 09 & 11.6 & 21.93 \\
\hline IC 0342 & 034648.5 & +680546 & $2 ! \prime 23 \times 1 " .76$ & 41.6 & 1430.61 & $1 "$ " $72 \times 1$ " 13 & 11.4 & 31.44 \\
\hline NGC 1482 & 035439.5 & -203007 & $3 ! \prime 25 \times 1 ! " 67$ & 16.8 & 419.18 & $2 ! " 42 \times 1 ! 33$ & 14.1 & 23.56 \\
\hline NGC 2146 & 061837.7 & +782125 & $2 . " 55 \times 1 " .55$ & 35.5 & 1214.57 & $1 " .93 \times 0 " .94$ & 14.9 & 44.68 \\
\hline NGC 2403 & 073650.0 & +653604 & $2 ! " 21 \times 1 " .53$ & 13.8 & 551.32 & $1 "$ " $88 \times 1 "$ " 15 & 9.8 & 24.41 \\
\hline Holmberg II & $\begin{array}{lll}08 & 19 & 13.3\end{array}$ & +704308 & $2 ! " 59 \times 1 " .67$ & 14.9 & 465.55 & $1 ! " 97 \times 1$ × 15 & 9.4 & 22.41 \\
\hline NGC 2798 & 091722.8 & +415958 & $2 ! " 13 \times 1 ! " 78$ & 14.6 & 520.66 & $1 "$ " $58 \times 1$ " 38 & 11.3 & 27.83 \\
\hline NGC 2841 & 092202.7 & +505836 & $2 ! " 11 \times 1{ }^{\prime \prime} 62$ & 13.3 & 526.88 & 1 ". $59 \times 1$ × 23 & 18.1 & 50.13 \\
\hline NGC 2976 & 094715.3 & +675500 & $2 ! " 83 \times 1 " .66$ & 14.4 & 413.49 & $2 ! \prime 02 \times 1 "$ "13 & 9.7 & 23.08 \\
\hline NGC 3049 & 095449.6 & +091617 & 2 "! $14 \times 1$ " 84 & 14.9 & 510.77 & $2 ! " 07 \times 1$ "! 13 & 12.2 & 27.98 \\
\hline NGC 3077 & 100319.1 & +684402 & $2 ! " 90 \times 1 . " 67$ & 14.4 & 402.59 & 1 ".98 × 1 " 12 & 10.2 & 24.82 \\
\hline NGC 3190 & 101805.6 & +214955 & $2 ! " 12 \times 1 "$ " 81 & 13.5 & 475.29 & $1 "$ " $61 \times 1$ " 26 & 8.5 & 22.63 \\
\hline NGC 3184 & 101816.7 & +412527 & $2 ! " 42 \times 1 "$ " 76 & 14.0 & 444.09 & $1 " .29 \times 1$ " 18 & 8.5 & 30.49 \\
\hline NGC 3198 & 101954.9 & +453259 & $2 ! " 25 \times 1$ "! 66 & 14.4 & 521.10 & 1 ". $34 \times 1$ " 17 & 8.5 & 29.06 \\
\hline IC 2574 & 102848.4 & +682802 & $2 ! " 96 \times 1$ × 64 & 14.2 & 393.53 & $2 ! " 03 \times 1 "$ × 14 & 8.8 & 20.73 \\
\hline NGC 3265 & 103106.7 & +284748 & $2 ! " 23 \times 1 "$ × 78 & 14.9 & 505.30 & 1 " $44 \times 1$ " 25 & 7.8 & 23.42 \\
\hline NGC 3351 & 104357.8 & +114214 & $2 ! \prime 00 \times 1 ! " 75$ & 18.4 & 708.89 & 1 " $95 \times 1$ × 67 & 15.3 & 25.34 \\
\hline NGC 3521 & 110548.9 & -000206 & 3 ". $07 \times 1$ " 95 & 19.9 & 448.93 & 1 "! $88 \times 1$ "! 19 & 16.4 & 39.79 \\
\hline NGC 3621 & 111816.0 & -324842 & $4 ! " 76 \times 1$ "! 53 & 15.7 & 291.11 & $4 ! " 17 \times 1$ " 08 & 8.5 & 10.16 \\
\hline NGC 3627 & 112015.0 & +125930 & $1 ! " 95 \times 1 ! " 77$ & 15.7 & 616.48 & 2 ". $36 \times 1$ × 97 & 14.0 & 16.23 \\
\hline NGC 3773 & 113813.0 & +120645 & $1 "$ "95 × 1 "! 76 & 13.1 & 518.26 & $1 "$ " $53 \times 1$ ×! 14 & 9.8 & 30.21 \\
\hline NGC 3938 & 115249.5 & +440714 & $3 ! \prime 13 \times 1 " ! 73$ & 15.1 & 376.61 & 1 ". $25 \times 1$ " 10 & 7.9 & 31.23 \\
\hline NGC 4254 & 121849.4 & +142459 & 1 ".99 × 1". 81 & 15.3 & 577.12 & 1 !" $53 \times 1$ "! 11 & 9.0 & 28.58 \\
\hline NGC 4321 & 122254.9 & +154921 & $1 "$ " $89 \times 1 "$ × 72 & 15.0 & 622.65 & $1 "$ "53 × 1 " 17 & 9.8 & 29.55 \\
\hline NGC 4536 & 123427.1 & +021117 & $2 ! " 16 \times 1 "$ " 86 & 15.5 & 524.37 & $2 ! " 44 \times 1 "$ " 45 & 10.2 & 15.58 \\
\hline NGC 4559 & 123557.7 & +275736 & 1 ". $75 \times 1$ ". 63 & 13.6 & 646.04 & $1 "$ " $32 \times 1 " .27$ & 9.0 & 29.13 \\
\hline NGC 4569 & 123649.8 & +130946 & $2 ! " 01 \times 1 "$ × 80 & 15.4 & 572.68 & 1 " $68 \times 1$ " 18 & 10.7 & 29.15 \\
\hline NGC 4579 & 123743.6 & +114902 & $2 ! \prime 12 \times 1 " .85$ & 23.4 & 807.73 & $1 " .72 \times 1 " ! 21$ & 50.9 & 132.87 \\
\hline NGC 4594 & 123959.4 & -113723 & $2 ! \prime 76 \times 1 ! " 74$ & 19.7 & 554.75 & $2 ! " 01 \times 1 . \prime 09$ & 13.4 & 32.88 \\
\hline NGC 4625 & 124152.4 & +411624 & $1 "$ " $73 \times 1$ × 56 & 13.5 & 677.51 & 1 ". $54 \times 1$ "! 17 & 9.7 & 29.19 \\
\hline NGC 4631 & 124205.9 & +323222 & $1 ! " 85 \times 1 ! " 76$ & 14.3 & 597.52 & $2 ! " 29 \times 1 "$ " 83 & 12.9 & 16.71 \\
\hline NGC 4725 & 125026.6 & +253006 & $1 " .85 \times 1 "$ × 76 & 13.5 & 561.64 & $1 ! 33 \times 1$ " 26 & 9.0 & 29.20 \\
\hline NGC 4736 & 125053.0 & +410714 & $1 " .72 \times 1 " .55$ & 13.9 & 706.09 & 2 ". $25 \times 2$ ". 06 & 13.4 & 15.67 \\
\hline NGC 4826 & 125643.9 & +214100 & $1 ! " 97 \times 1 ! " 75$ & 14.3 & 559.38 & 1 " $46 \times 1$ "! 33 & 10.1 & 28.20 \\
\hline NGC 5055 & $13 \quad 1549.2$ & +420149 & $1 "$ " $76 \times 1$ 1" 60 & 12.8 & 619.07 & 1 !" $51 \times 1$ "! 17 & 9.7 & 29.58 \\
\hline NGC 5194 & 132952.7 & +471143 & 1 ". $79 \times 1$ ×. 57 & 17.0 & 820.75 & $1 "$ " $54 \times 1$ " 15 & 9.3 & 28.43 \\
\hline NGC 5398 & 140120.2 & -330409 & $6 ! " 62 \times 1 "$ " 76 & 20.6 & 239.46 & $3 ! " 98 \times 1$ " 03 & 8.4 & 11.09 \\
\hline NGC 5457 & 140312.6 & +542057 & $1 ! " 82 \times 1 "$ × 67 & 13.9 & 619.05 & 1 ". $55 \times 1$ "! 10 & 8.6 & 27.29 \\
\hline NGC 5474 & 140501.3 & +533944 & 1 ". $83 \times 1$ × 71 & 14.3 & 616.05 & 1 ". $49 \times 1$ " 07 & 11.9 & 40.40 \\
\hline NGC 5713 & 144011.3 & -001727 & $2 ! " 55 \times 1 " .77$ & 17.6 & 526.76 & $2 ! " 35 \times 1 "$ " 80 & 15.4 & 19.67 \\
\hline NGC 5866 & 150629.5 & +554548 & $1 " \prime 92 \times 1 " \prime 63$ & 13.8 & 595.03 & $1 "$ " $62 \times 1$ "! 20 & 15.4 & 42.92 \\
\hline NGC 6946 & 203452.3 & +600914 & 2 "! $03 \times 1$ × 64 & 16.1 & 655.75 & 2 "! $15 \times 1$ "! 12 & 9.5 & 21.44 \\
\hline NGC 7331 & 223704.1 & +342456 & 1 ". $82 \times 1$ " 67 & 15.1 & 672.97 & $1 " .51 \times 1 " ! 21$ & 10.0 & 29.80 \\
\hline NGC 7793 & 235749.2 & -323524 & $4 ! " 77 \times 1 ! " 62$ & 14.0 & 245.06 & $4 ! " 47 \times 1$ " 08 & 9.4 & 10.50 \\
\hline
\end{tabular}

Note. See Murphy et al. (2018a) for the $33 \mathrm{GHz}$ imaging characteristics.

\subsection{Interferometric Imaging}

Calibrated VLA measurement sets for each source were imaged using the task TCLEAN in CASA version 4.6.0. For some cases, the $\mathrm{K} a$-band images contain data from observations taken during both the $11 \mathrm{~B}$ and $13 \mathrm{~A}$ semesters but are heavily weighted by the $13 \mathrm{~A}$ semester observations, as those include significantly more data. The mode of TCLEAN was set to multifrequency synthesis (MFS; Conway et al. 1990;
Sault \& Wieringa 1994). We chose a pixel scale of 0"' 2 for all three bands and adopted Briggs weighting with ROBUST $=0.5$ and NTERMS $=2$. This allows the cleaning procedure to also model the spectral index variations on the sky. Although this procedure utilizes the large fractional bandwidths of each observation to generate in-band spectral index maps, we do not use them in our analysis given that the signal-to-noise ratio $(\mathrm{S} / \mathrm{N})$ of our sources is typically too low for them to be 
Table 3

Extranuclear Source Positions and Imaging Characteristics

\begin{tabular}{|c|c|c|c|c|c|c|c|c|}
\hline \multirow[b]{2}{*}{ Enuc. ID } & \multirow[b]{2}{*}{$\begin{array}{l}\text { R.A. } \\
\text { (J2000) }\end{array}$} & \multirow[b]{2}{*}{$\begin{array}{l}\text { Decl. } \\
\text { (J2000) }\end{array}$} & \multicolumn{3}{|c|}{$3 \mathrm{GHz}$} & \multicolumn{3}{|c|}{$15 \mathrm{GHz}$} \\
\hline & & & $\begin{array}{l}\text { Synthesized } \\
\text { Beam }\end{array}$ & $\begin{array}{c}\sigma \\
\left(\mu \mathrm{Jy} \mathrm{bm}^{-1}\right)\end{array}$ & $\begin{array}{c}\sigma_{T_{\mathrm{b}}} \\
(\mathrm{mK})\end{array}$ & $\begin{array}{l}\text { Synthesized } \\
\text { Beam }\end{array}$ & $\begin{array}{c}\sigma \\
\left(\mu \mathrm{Jy} \mathrm{bm}^{-1}\right)\end{array}$ & $\begin{array}{c}\sigma_{T_{\mathrm{b}}} \\
(\mathrm{mK})\end{array}$ \\
\hline NGC 0628 Enuc. 1 & 013645.1 & +154751 & $1 ! .96 \times 1 ! .78$ & 14.0 & 542.77 & $1 ! 56 \times 1$ !. 21 & 9.8 & 28.16 \\
\hline NGC 0628 Enuc. 2 & 013637.5 & +154512 & $1 ! .96 \times 1 ! .78$ & 14.0 & 544.62 & $1 ! .57 \times 1 ! .22$ & 9.6 & 27.27 \\
\hline NGC 0628 Enuc. 3 & 013638.8 & +154425 & 1 !" $96 \times 1$ " 78 & 14.0 & 542.12 & $1 ! .57 \times 1$ !" 23 & 9.6 & 26.82 \\
\hline NGC 0628 Enuc. 4 & 013635.5 & +155011 & $1 "$ "96 × ${ }^{\prime \prime} .78$ & 13.8 & 537.98 & $1 "$ " $56 \times 1$ ". 20 & 8.0 & 23.07 \\
\hline NGC 1097 Enuc. 1 & 024623.9 & -301751 & $5 ! .76 \times 1 ! " 80$ & 41.2 & 537.94 & $3 ! " 76 \times 0$ ".95 & 11.8 & 17.84 \\
\hline NGC 1097 Enuc. 2 & 024614.4 & -301505 & $5 ! 76 \times 1$ !" 80 & 38.3 & 500.07 & 3 !" $80 \times 0$ ". 98 & 11.4 & 16.42 \\
\hline NGC 2403 Enuc. 1 & 073645.5 & +653700 & $2 ! .21 \times 1 " .53$ & 13.7 & 548.00 & 1 !" $86 \times 1$." 16 & 9.4 & 23.61 \\
\hline NGC 2403 Enuc. 2 & 073652.7 & +653646 & $2 ! .21 \times 1 ! .53$ & 13.7 & 547.71 & $1 ! .85 \times 1$ !! 15 & 9.5 & 23.96 \\
\hline NGC 2403 Enuc. 3 & 073706.9 & +653639 & $2 . .21 \times 1 " .53$ & 13.7 & 548.41 & 1 !" $84 \times 1$ !" 16 & 9.7 & 24.51 \\
\hline NGC 2403 Enuc. 4 & 073717.9 & +653346 & $2 ! .21 \times 1 . .53$ & 13.7 & 546.14 & $1 ! " 80 \times 1{ }^{\prime \prime} 15$ & 9.5 & 24.86 \\
\hline NGC 2403 Enuc. 5 & 073619.5 & +653704 & $2 ! .21 \times 1 . .53$ & 13.5 & 541.72 & $1 " .79 \times 1$ !" 15 & 9.5 & 24.84 \\
\hline NGC 2403 Enuc. 6 & 073628.5 & +653350 & $2 ! .21 \times 1 "$ ! 53 & 13.5 & 541.23 & $1 ! .77 \times 1{ }^{\prime \prime} 13$ & 9.7 & 26.15 \\
\hline NGC 2976 Enuc. 1 & 094707.8 & +675552 & 2 ". $83 \times 1$ ". 66 & 14.5 & 415.03 & $1 "$.99 × ${ }^{\prime \prime} 13$ & 9.8 & 23.62 \\
\hline NGC 2976 Enuc. 2 & 094724.1 & +675357 & 2 ". $83 \times 1 " .66$ & 14.4 & 412.17 & $1 " .97 \times 1$ ×. 13 & 10.0 & 24.19 \\
\hline NGC 3521 Enuc. 1 & 110546.3 & -000410 & $3 ! .07 \times 1$ "! 95 & 19.4 & 436.89 & $1 ! 92 \times 1 " .22$ & 14.0 & 32.41 \\
\hline NGC 3521 Enuc. 2 & 110549.9 & -000340 & $3 ! .07 \times 1 !$ ! 95 & 19.8 & 445.88 & 2 !" $05 \times 1$ !! 11 & 11.7 & 27.66 \\
\hline NGC 3521 Enuc. 3 & 110547.6 & +000033 & $3 ! .07 \times 1 !$ "95 & 18.9 & 425.62 & $2 ! " 21 \times 1 ! ! 13$ & 11.6 & 25.02 \\
\hline NGC 3627 Enuc. 1 & 112016.2 & +125750 & $1.95 \times 1$ × 77 & 15.4 & 604.26 & $1 " .50 \times 1$ "! 22 & 9.0 & 26.70 \\
\hline NGC 3627 Enuc. 2 & 112016.3 & +125844 & 1 !"95 × 1.77 & 15.7 & 614.25 & $1 " .92 \times 1{ }^{\prime \prime} 41$ & 15.7 & 31.48 \\
\hline NGC 3627 Enuc. 3 & 112016.0 & +125952 & 1 !" $95 \times 1$ ×. 77 & 15.7 & 616.15 & 1 !. $51 \times 1$ !! 17 & 13.4 & 40.92 \\
\hline NGC 3938 Enuc. 1 & 115246.4 & +440701 & $3 ! ! 13 \times 1 !$ ! 73 & 15.0 & 374.91 & $1 ! 27 \times 1 ! ! 12$ & 8.3 & 31.38 \\
\hline NGC 3938 Enuc. 2 & 115300.0 & +440755 & $3 ! \prime 13 \times 1 "$ " 73 & 14.8 & 368.03 & $1 ! 27 \times 1 "$. 12 & 8.3 & 31.40 \\
\hline NGC 4254 Enuc. 1 & 121849.1 & +142359 & $1 ! .99 \times 1 " .81$ & 15.1 & 567.30 & $1 " .55 \times 1 !$. 14 & 8.5 & 26.05 \\
\hline NGC 4254 Enuc. 2 & 121844.6 & +142425 & $1 "$ "99 × 1"! 81 & 15.3 & 575.22 & $1 "$ " $51 \times 1$ !" 16 & 9.6 & 29.55 \\
\hline NGC 4321 Enuc. 1 & 122258.9 & +154935 & $1 ! .89 \times 1 ! .72$ & 14.9 & 620.02 & $1 ! .53 \times 1 ! ! 18$ & 9.8 & 29.30 \\
\hline NGC 4321 Enuc. 2 & 122249.8 & +155029 & $1 ! .89 \times 1 ! .72$ & 14.9 & 618.78 & $1 " .52 \times 1$ !" 18 & 9.9 & 29.98 \\
\hline NGC 4631 Enuc. 1 & 124140.8 & +323151 & $1 ! .87 \times 1$ "! 75 & 14.0 & 577.02 & 1 !" $66 \times 1$ ×. 27 & 9.1 & 23.44 \\
\hline NGC 4631 Enuc. 2 & 124221.3 & +323307 & $1 ! .85 \times 1 ! .76$ & 13.9 & 578.32 & 1 !" $61 \times 1{ }^{\prime \prime} 28$ & 9.0 & 23.48 \\
\hline NGC 4736 Enuc. 1 & 125056.2 & +410720 & $1 " .72 \times 1 " .55$ & 13.9 & 705.86 & 1 !" $54 \times 1$ !! 17 & 12.9 & 38.89 \\
\hline NGC 5055 Enuc. 1 & 131558.0 & +420026 & 1 !" $76 \times 1$ !" 60 & 13.1 & 630.58 & $1 ! 50 \times 1 !$ ! 17 & 9.9 & 30.50 \\
\hline NGC 5194 Enuc. 1 & 132953.1 & +471240 & $1 "$ " $79 \times 1 "$ " 57 & 16.8 & 810.49 & $1 ! .53 \times 1 !$ ! 14 & 9.1 & 28.20 \\
\hline NGC 5194 Enuc. 2 & 132944.1 & +471021 & $1 !$ " $74 \times 1$ ". 52 & 17.0 & 863.15 & $1 " .52 \times 1 !$ ! 14 & 8.9 & 27.62 \\
\hline NGC 5194 Enuc. 3 & 132944.6 & +470955 & $1 "$ " $74 \times 1$ " 52 & 16.9 & 858.25 & $1 "$ " $52 \times 1 "$ " 14 & 9.1 & 28.37 \\
\hline NGC 5194 Enuc. 4 & 132956.2 & +471407 & $1 " .79 \times 1 " .57$ & 16.0 & 768.85 & $1 " .52 \times 1 !$ " 14 & 9.5 & 29.61 \\
\hline NGC 5194 Enuc. 5 & 132959.6 & +471401 & 1 ". $79 \times 1$ ". 57 & 16.1 & 773.77 & $1 " .52 \times 1{ }^{\prime \prime} 14$ & 9.7 & 30.21 \\
\hline NGC 5194 Enuc. 6 & 132939.5 & +470835 & $1 ! 74 \times 1$ ×. 52 & 15.8 & 805.61 & 1 !" $51 \times 1$ "! 12 & 8.7 & 27.81 \\
\hline NGC 5194 Enuc. 7 & 133002.5 & +470952 & $1 ! .74 \times 1 "$ " 52 & 17.8 & 904.22 & $1 ! .52 \times 1 ! ! 11$ & 9.3 & 29.80 \\
\hline NGC 5194 Enuc. 8 & 133001.6 & +471252 & $1 " .79 \times 1 " .57$ & 16.7 & 803.48 & 1 !" $58 \times 1$ !" 18 & 9.7 & 28.04 \\
\hline NGC 5194 Enuc. 9 & 132959.9 & +471112 & $1 ! .79 \times 1 ! .57$ & 17.0 & 819.52 & 1 !" $59 \times 1$ !" 16 & 10.7 & 31.20 \\
\hline NGC 5194 Enuc. 10 & 132956.7 & +471046 & $1 !$ " $74 \times 1$ ". 52 & 18.1 & 921.23 & $1 " .59 \times 1 "$ " 16 & 10.8 & 31.55 \\
\hline NGC 5194 Enuc. 11 & 132949.7 & +471329 & 1 ". $79 \times 1$ ". 57 & 16.3 & 783.23 & $1 ! .57 \times 1 ! .21$ & 12.8 & 36.27 \\
\hline NGC 5457 Enuc. 1 & 140310.2 & +542058 & 1 ". $82 \times 1$ ". 67 & 13.8 & 615.51 & $1 " .55 \times 1 "$ " 10 & 8.4 & 26.65 \\
\hline NGC 5457 Enuc. 2 & 140255.0 & +542227 & $1 ! .81 \times 1 "$ " 63 & 14.1 & 643.39 & $1 "$ " $54 \times 1$ ". 09 & 8.5 & 27.14 \\
\hline NGC 5457 Enuc. 3 & 140341.3 & +541905 & $1 ! .82 \times 1$ !" 67 & 14.1 & 627.94 & $4 ! .68 \times 2 . .20$ & 25.0 & 13.13 \\
\hline NGC 5457 Enuc. 4 & 140353.1 & +542206 & $1 " .82 \times 1 " .73$ & 13.8 & 590.56 & $1 ! .54 \times 1 !$ " 08 & 9.5 & 30.71 \\
\hline NGC 5457 Enuc. 5 & 140301.1 & +541429 & $1 "$ " $81 \times 1 "$ " 63 & 13.8 & 629.72 & $1 ! 49 \times 1 ! 07$ & 10.8 & 36.48 \\
\hline NGC 5457 Enuc. 6 & 140228.1 & +541626 & 1 !" $86 \times 1$ ×. 67 & 13.7 & 596.38 & 1 !. $53 \times 1$ !" 06 & 11.9 & 39.68 \\
\hline NGC 5457 Enuc. 7 & 140429.3 & +542346 & $1 ! .82 \times 1 ! .73$ & 13.7 & 589.02 & 1 !" $50 \times 1{ }^{\prime \prime} 04$ & 13.0 & 44.82 \\
\hline NGC 5713 Enuc. 1 & 144012.1 & -001747 & $2 ! .55 \times 1 ! .77$ & 17.6 & 525.60 & $2 ! " 35 \times 1$ ". 80 & 15.9 & 20.36 \\
\hline NGC 5713 Enuc. 2 & 144010.5 & -001747 & $2 . .55 \times 1 " .77$ & 17.6 & 527.29 & 2 ". $35 \times 1$ ". 80 & 15.9 & 20.36 \\
\hline NGC 6946 Enuc. 1 & 203516.6 & +601057 & $2 ! .03 \times 1 " .64$ & 15.2 & 616.55 & $2 ! .07 \times 1 " .10$ & 8.3 & 19.75 \\
\hline NGC 6946 Enuc. 2 & 203525.1 & +601003 & $2 ! .03 \times 1 ! .64$ & 15.0 & 609.01 & $2 ! 00 \times 1 " .09$ & 9.8 & 24.32 \\
\hline NGC 6946 Enuc. 3 & 203452.2 & +601241 & $2 ! " 03 \times 1 ! .64$ & 15.0 & 608.66 & $2 ! 09 \times 1 " .09$ & 8.7 & 20.65 \\
\hline NGC 6946 Enuc. 4 & 203419.4 & +601009 & $2 ! .03 \times 1 " .64$ & 14.8 & 603.01 & $2 ! .45 \times 1 " .90$ & 10.1 & 11.78 \\
\hline NGC 6946 Enuc. 5 & 203439.0 & +600453 & $2 ! " 06 \times 1$ ". 63 & 15.1 & 604.38 & $1 ! " 91 \times 1 ! ! 11$ & 8.8 & 22.34 \\
\hline NGC 6946 Enuc. 6 & 203506.0 & +601101 & $2 ! .03 \times 1 . " 64$ & 15.7 & 636.59 & $1 ! .95 \times 1 ! ! 11$ & 8.6 & 21.28 \\
\hline NGC 6946 Enuc. 7 & 203511.2 & +600860 & $2 ! .03 \times 1$ ". 64 & 15.7 & 636.59 & $2 ! .14 \times 1 ! ! 13$ & 8.8 & 19.64 \\
\hline NGC 6946 Enuc. 8 & 203432.2 & +601020 & $2 ! .05 \times 1 ! .63$ & 15.8 & 639.98 & $1 ! 99 \times 1 "$ " 11 & 8.7 & 21.20 \\
\hline NGC 6946 Enuc. 9 & 203512.7 & +600853 & 2 ". $03 \times 1$ ". 64 & 15.7 & 636.59 & 2 ". $14 \times 1$ ". 13 & 8.8 & 19.64 \\
\hline NGC 7793 Enuc. 1 & 235748.8 & -323659 & $4 ! " 77 \times 1$ !" 62 & 13.9 & 243.82 & $4 ! .62 \times 1 ! .09$ & 9.6 & 10.26 \\
\hline NGC 7793 Enuc. 2 & 235756.1 & -323540 & $4 ! " 77 \times 1 !$ " 62 & 14.0 & 244.33 & $4 ! " 71 \times 1 !$ ! 11 & 9.8 & 10.09 \\
\hline NGC 7793 Enuc. 3 & 235748.8 & -323453 & $4 ! .77 \times 1$ !" 62 & 14.0 & 245.29 & $4 ! " 47 \times 1$ !! 08 & 8.8 & 9.84 \\
\hline
\end{tabular}

Note. See Murphy et al. (2018a) for the $33 \mathrm{GHz}$ imaging characteristics.

reliable. To help deconvolve extended low-intensity emission, we took advantage of the multiscale CLEAN option (Cornwell 2008; Rau \& Cornwell 2011) in CASA, searching for structures with scales $\sim 1$ and 3 times the FWHM of the synthesized beam. The choice of our final imaging parameters was the result of extensive experimentation to identify values 
that yielded the best combination of brightness temperature sensitivity and reduction of artifacts resulting from strong sidelobes in the naturally weighted beam for these snapshotlike observations.

A primary beam correction was applied using the CASA task IMPBCOR before analyzing the images. The primary-beamcorrected continuum images at $3-33 \mathrm{GHz}$ are shown in Figure 1. The FWHM of the synthesized beam and the corresponding point-source and brightness temperature sensitivities for each image are given in Tables 2 and 3. Finally, in order to accurately compare the flux density measured for each star-forming region across the full $3-33 \mathrm{GHz}$ frequency range, we use the CASA task IMSMOOTH to match all VLA images for each pointing to a common circularized, Gaussian beam corresponding to the lowest angular resolution among all three frequency bands scaled by a factor of $\sqrt{2} \times 0$ "! 2 (i.e., the pixel scale). This scaling factor eliminates cases for the convolution kernels used by IMSMOOTH to have length zero in any axis in units of pixels. We additionally crop all images to a common field of view (FOV) equal to the FOV of our $33 \mathrm{GHz}$ observations (i.e., a primary beam FWHM of 44").

\section{Ancillary Data and Photometry}

In this section we provide a description of ancillary data used, as well as our procedure for extracting consistent aperture photometry across the full suite of multiwavelength data available for galaxies in the SFRS.

\subsection{Ancillary Data}

GALEX far-UV (FUV; $1528 \AA$ ) and near-UV (NUV; $2271 \AA$ ) data were taken from the GALEX Large Galaxy Atlas (Seibert 2007). The calibration uncertainty for these data is $\sim 15 \%$ in both bands. One galaxy, NGC 1377, does not have existing NUV or FUV imaging.

The $\mathrm{H} \alpha$ data used in this analysis are taken from the compilation presented in Leroy et al. (2012), where details about the data quality and preparation (e.g., correction for [N II] emission) can be found, as well as the SINGS archive. All $\mathrm{H} \alpha$ images were then further corrected for foreground stars. The typical resolution of the seeing-limited $\mathrm{H} \alpha$ images is $\sim 1^{\prime \prime}-2^{\prime \prime}$, and the calibration uncertainty among these maps is taken to be $\sim 20 \%$. Two galaxies, IC 342 and NGC 2146, do not have $\mathrm{H} \alpha$ imaging from SINGS.

Archival Spitzer $8 \mu \mathrm{m}$ and $24 \mu \mathrm{m}$ data were largely taken from the SINGS and Local Volume Legacy (LVL) legacy programs and have a calibration uncertainty of $\sim 5 \%$. Details on the associated observation strategies and data reduction steps can be found in Dale et al. (2007, 2009). Two galaxies, IC 342 and NGC 2146, were not a part of SINGS or LVL; their $24 \mu \mathrm{m}$ imaging comes from Engelbracht et al. (2008).

Finally, in order to account for the significant differences between the point-spread functions (PSFs) of the various telescopes used in this analysis, we implement the convolution kernels presented in Aniano et al. (2011) to convolve the instrumental PSF for each image, and we produce a corrected Gaussian beam of $7^{\prime \prime}$ at each wavelength.

\subsection{Region Identification and Aperture Photometry}

To identify and characterize potential star-forming regions in the SFRS, we start by searching within an area that is equal to twice the FWHM of the VLA primary beam at $33 \mathrm{GHz}\left(\sim 160^{\prime \prime}\right)$. Any region visually identified in least one radio band is retained for further investigation. The locations of all radio-identified sources were compared with the $8 \mu \mathrm{m}$ maps of each galaxy to determine whether the source has a corresponding detection in the mid-IR and is thus likely associated with a star-forming region. Sources characterized as potential background galaxies have no obvious $8 \mu \mathrm{m}$ counterpart, and very rarely a $33 \mathrm{GHz}$ counterpart. In total, we visually identified 389 regions for which we perform aperture photometry to determine those that are statistically significant detections. Of the 389 sources visually identified, 377 had a statistically significant detection (i.e., $\mathrm{S} / \mathrm{N}>3$ ) in at least one band, which is given in Table 4. For completeness, the remaining 12 sources are still labeled in the panels of Figure 1 to demonstrate the full identification process we utilized. Figures A1-A15 with the remaining three-panel images for all galaxies in the SFRS are presented in the Appendix.

Using the CASA task IMSTAT, we measured and report the 3-33 GHz flux densities for each region. The sizes of the apertures were hand selected to fully encompass the visible extent of the $3-33 \mathrm{GHz}$ radio flux density of each region, with an additional constraint of having a diameter equal to or larger than the FWHM of the synthesized beam for that pointing. We do not apply aperture corrections to our photometry given that we both convolve all images for a given pointing to a common beam and use the same-sized aperture at all wavelengths. Photometric uncertainties were conservatively estimated by taking the empirically measured noise from empty regions in each non-primary beam corrected image, applying the empirical primary beam correction based on Equation (4) in EVLA Memo 195, and scaling by the ratio of the synthesized beam area to the adopted aperture area. This noise is then added in quadrature with the VLA calibration uncertainty $(\sim 3 \%$; Perley \& Butler 2013). The median size of the apertures used for all 377 sources is $164 \pm 6.3 \mathrm{pc}$ with a median absolute deviation of $97 \mathrm{pc}$. The aperture sizes used for each identified region are given in Table 4.

We additionally carried out photometry on the full VLA, GALEX, $\mathrm{H} \alpha$, and Spitzer/MIPS $24 \mu \mathrm{m}$ data sets after matching their resolution ( $7^{\prime \prime}$ FWHM), cropping each image to a common FOV, and regridding to a common pixel scale $(0.2)$. In total, we identify 180 discrete sources, and critical apertures of $7^{\prime \prime}$ were used in all cases. Unlike our native-resolution photometry, we report sources with upper limits in all radio bands if a statistically significant detection exists in our ancillary GALEX and Spitzer imaging. The median size of the apertures used at $7^{\prime \prime}$ resolution is $259 \pm 7.2 \mathrm{pc}$ with a median absolute deviation of $73 \mathrm{pc}$. The UV and $\mathrm{H} \alpha$ photometry of each region was corrected for Milky Way extinction using Schlegel et al. (1998) assuming $A_{V} / E(B-V)=3.1$ and the modeled extinction curves of Weingartner \& Draine (2001) and Draine (2003). The results from our radio photometry are given in Table 5 . The GALEX, $\mathrm{H} \alpha$, and Spitzer/MIPS $24 \mu \mathrm{m}$ photometry results, which are presented in Table A1, are not used directly in the present analysis but will be utilized for further studies.

Finally, in order to account for and remove any diffuse emission component that is most likely unassociated with the most recent star formation activity in the disks of these galaxies, we measure a local background value within the vicinity of each star-forming region. The local background was measured by placing an annulus a distance of 1.5 times the synthesized beam FWHM away from the center of the source position in both the full-resolution and smoothed maps. The median surface brightness within this annulus was then 


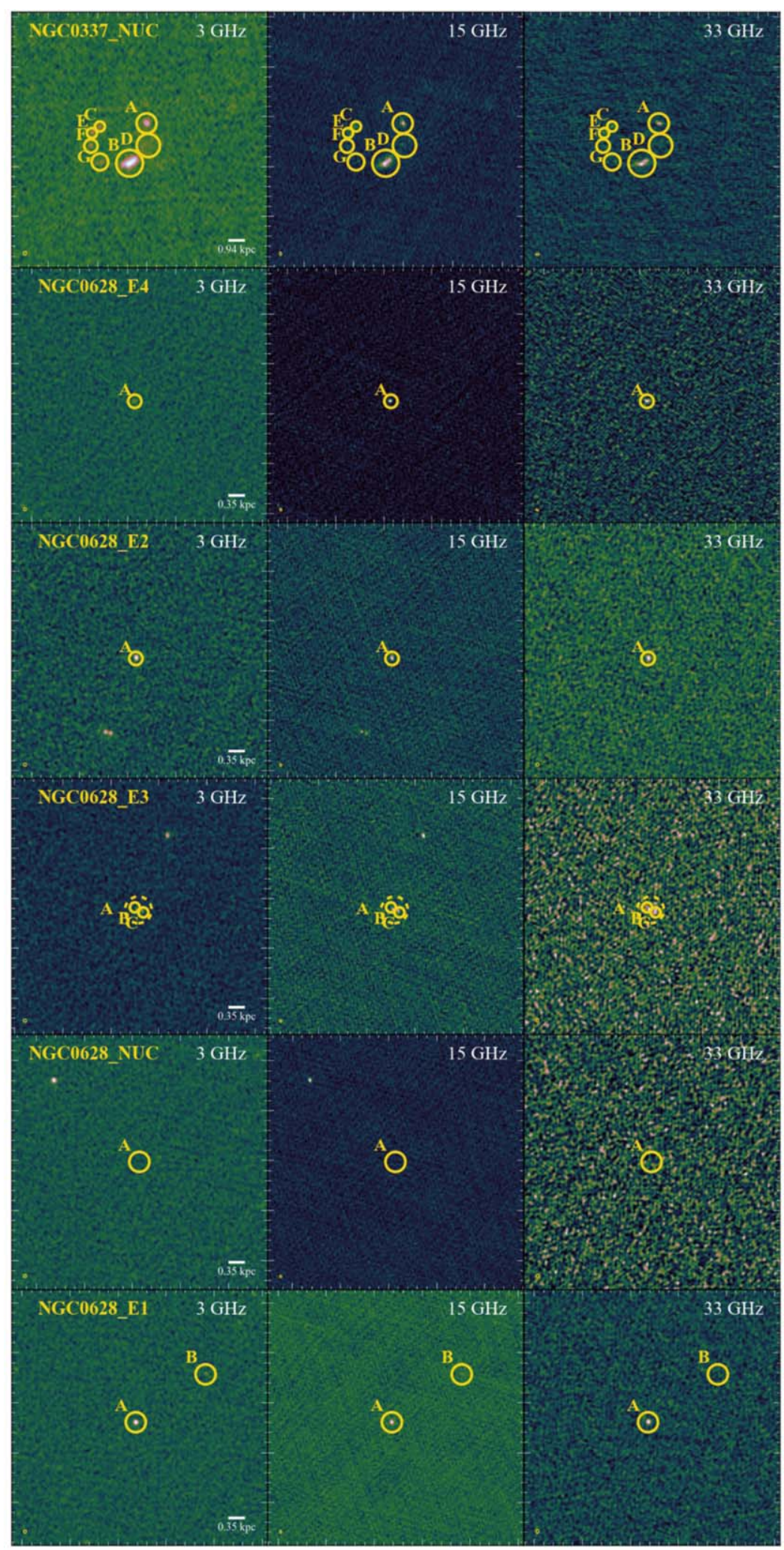

Figure 1. Three-panel images for all 56 galaxies in the SFRS showing the combination of our $3 \mathrm{GHz}$ (left), $15 \mathrm{GHz}$ (middle), and $33 \mathrm{GHz}$ (right) observations. The color scale (Green 2011) is set to one of four power-law stretches: $\left[a\left(p-p_{\min }\right) /\left(p_{\max }-p_{\min }\right)\right]$, where $p$ is the pixel value and $a=1 / 3,0.5,1.0$, and 2.0 . A cube-root stretch of $a=1 / 3$ was used when the brightest pixel in the image had an $\mathrm{S} / \mathrm{N}>500$. A square-root stretch of $a=0.5$ was used when the brightest pixel in the image had $50<\mathrm{S} / \mathrm{N}<500$. A linear stretch was used when the brightest pixel was between $10<\mathrm{S} / \mathrm{N}<50$, and a square stretch was used when the brightest pixel had an $\mathrm{S} / \mathrm{N}<10$. A scale bar of $10^{\prime \prime}$ is also given in the lower right corner of each panel. To distinguish between individual sources identified in the full-resolution and $7^{\prime \prime}$ smoothed maps, we use uppercase and lowercase letters as part of their names for reporting photometry in Tables 4 and 5, respectfully. An extended version of this figure for all remaining sources is available in the Appendix. 
Table 4

Region Photometry and Derived Parameters

\begin{tabular}{|c|c|c|c|c|c|c|c|c|c|}
\hline Source ID & $\begin{array}{c}\text { R.A. } \\
\text { (J2000) }\end{array}$ & $\begin{array}{c}\text { Decl. } \\
\text { (J2000) }\end{array}$ & $\begin{array}{l}S_{3 \mathrm{GHz}} \\
(\mathrm{mJy})\end{array}$ & $\begin{array}{c}S_{15 \mathrm{GHz}} \\
(\mathrm{mJy})\end{array}$ & $\begin{array}{c}S_{33 \mathrm{GHz}} \\
(\mathrm{mJy})\end{array}$ & $\begin{array}{l}d_{\mathrm{ap}} \\
(\mathrm{pc})\end{array}$ & $\begin{array}{c}r_{\mathrm{G}} \\
(\mathrm{kpc})\end{array}$ & $\alpha$ & $f_{\mathrm{T}}^{33 \mathrm{GHz}}$ \\
\hline \multicolumn{10}{|c|}{ Star-forming Regions } \\
\hline NGC 0337 D & 005949.94 & -073447.60 & $1.19 \pm 0.07$ & $0.77 \pm 0.04$ & $0.31 \pm 0.05$ & 655.0 & 0.921 & $-0.335 \pm 0.042$ & $0.841 \pm 0.038$ \\
\hline NGC 0337 A & 005950.01 & -073434.33 & $0.99 \pm 0.06$ & $0.70 \pm 0.03$ & $0.28 \pm 0.05$ & 561.0 & 0.881 & $-0.278 \pm 0.042$ & $0.888 \pm 0.033$ \\
\hline NGC 0337 B & 005950.69 & -073458.26 & $4.94 \pm 0.07$ & $2.66 \pm 0.04$ & $1.80 \pm 0.07$ & 749.0 & 2.041 & $-0.399 \pm 0.012$ & $0.780 \pm 0.012$ \\
\hline NGC $0337 \mathrm{C}$ & 005951.89 & -073436.20 & $0.30 \pm 0.03$ & $0.29 \pm 0.02$ & $0.21 \pm 0.03$ & 281.0 & 3.735 & $-0.076 \pm 0.060$ & $1.000 \pm 0.028$ \\
\hline NGC 0337 G & 005951.89 & -073457.50 & $0.36 \pm 0.05$ & $0.19 \pm 0.03$ & $0.17 \pm 0.05$ & 468.0 & 3.123 & $-0.358 \pm 0.102$ & $0.820 \pm 0.096$ \\
\hline NGC $0337 \mathrm{E}$ & 005952.22 & -073440.10 & $0.28 \pm 0.03$ & $0.27 \pm 0.02$ & $0.30 \pm 0.03$ & 281.0 & 4.048 & $0.022 \pm 0.059$ & $1.000 \pm 0.022$ \\
\hline NGC $0337 \mathrm{~F}$ & 005952.26 & -073447.90 & $0.28 \pm 0.04$ & $0.19 \pm 0.02$ & $0.22 \pm 0.04$ & 374.0 & 3.753 & $-0.159 \pm 0.087$ & $0.968 \pm 0.051$ \\
\hline NGC 0628 Enuc. 4 & 013635.69 & +155007.22 & $0.27 \pm 0.03$ & $0.26 \pm 0.02$ & $0.23 \pm 0.03$ & 140.0 & 7.614 & $-0.043 \pm 0.065$ & $1.000 \pm 0.029$ \\
\hline NGC 0628 Enuc. 2 & 013637.64 & +154507.19 & $0.51 \pm 0.03$ & $0.35 \pm 0.02$ & $0.29 \pm 0.04$ & 140.0 & 4.468 & $-0.233 \pm 0.044$ & $0.921 \pm 0.031$ \\
\hline NGC 0628 Enuc. $3 \mathrm{C}$ & 013638.63 & +154421.40 & $0.28 \pm 0.02$ & $0.21 \pm 0.02$ & $0.23 \pm 0.04$ & 105.0 & 5.798 & $-0.149 \pm 0.057$ & $0.974 \pm 0.033$ \\
\hline NGC 0628 Enuc. $3 \mathrm{~A}^{\mathrm{a}}$ & 013638.84 & +154422.90 & $0.51 \pm 0.06$ & $0.36 \pm 0.04$ & $0.51 \pm 0.10$ & 279.0 & 5.724 & $-0.098 \pm 0.080$ & $1.000 \pm 0.040$ \\
\hline NGC 0628 Enuc. 3 B & 013638.99 & +154424.40 & $0.29 \pm 0.02$ & $0.20 \pm 0.02$ & $0.22 \pm 0.04$ & 105.0 & 5.657 & $-0.195 \pm 0.058$ & $0.946 \pm 0.037$ \\
\hline NGC 0628 Enuc. $1 \mathrm{~B}$ & 13642.340 & +154816.84 & $<0.13$ & $0.41 \pm 0.04$ & $<0.66$ & 209.0 & 2.695 & $\cdots$ & $\cdots$ \\
\hline NGC 0628 Enuc. $1 \mathrm{~A}$ & 013645.25 & +154748.06 & $0.27 \pm 0.04$ & $0.26 \pm 0.04$ & $0.26 \pm 0.07$ & 209.0 & 2.466 & $-0.010 \pm 0.111$ & $1.000 \pm 0.045$ \\
\hline NGC 0855 C & 021403.56 & +275238.30 & $1.81 \pm 0.06$ & $1.06 \pm 0.04$ & $0.74 \pm 0.07$ & 377.0 & 0.072 & $-0.346 \pm 0.027$ & $0.831 \pm 0.025$ \\
\hline NGC $0855 \mathrm{~A}^{\mathrm{a}}$ & 021403.80 & +275237.85 & $2.27 \pm 0.10$ & $1.55 \pm 0.06$ & $0.87 \pm 0.11$ & 613.0 & 0.305 & $-0.279 \pm 0.034$ & $0.888 \pm 0.026$ \\
\hline NGC 0855 B & 021404.36 & +275236.80 & $0.37 \pm 0.03$ & $0.24 \pm 0.02$ & $0.19 \pm 0.03$ & 189.0 & 0.910 & $-0.289 \pm 0.062$ & $0.880 \pm 0.049$ \\
\hline NGC 0925 D & 022715.981 & +333338.58 & $0.25 \pm 0.02$ & $0.24 \pm 0.02$ & $<0.75$ & 133.0 & 5.510 & $-0.018 \pm 0.082$ & $1.000 \pm 0.034$ \\
\hline NGC 0925 A & 022717.00 & +333443.00 & $0.10 \pm 0.03$ & $<0.06$ & $<0.09$ & 177.0 & 0.183 & $\cdots$ & $\cdots$ \\
\hline NGC 0925 B & 022720.62 & +33 3429.80 & $0.22 \pm 0.02$ & $0.17 \pm 0.02$ & $<0.21$ & 133.0 & 2.097 & $-0.174 \pm 0.095$ & $0.959 \pm 0.057$ \\
\hline NGC 1097 Enuc. 2 & 024614.40 & -301504.80 & $1.32 \pm 0.25$ & $<0.16$ & $0.26 \pm 0.08$ & 138.0 & 7.378 & $-0.684 \pm 0.147$ & $0.358 \pm 0.301$ \\
\hline NGC 1097 D & 024618.34 & -301631.00 & $11.86 \pm 0.10$ & $2.97 \pm 0.04$ & $2.23 \pm 0.07$ & 275.0 & 0.813 & $-0.809 \pm 0.009$ & $0.059 \pm 0.024$ \\
\hline NGC 1097 B & 024618.96 & -301629.20 & $7.10 \pm 0.10$ & $3.35 \pm 0.04$ & $3.03 \pm 0.07$ & 275.0 & 0.052 & $-0.409 \pm 0.010$ & $0.769 \pm 0.010$ \\
\hline NGC $1097 \mathrm{~A}^{\mathrm{a}}$ & 024618.96 & -301629.20 & $104.78 \pm 0.35$ & $28.54 \pm 0.15$ & $21.13 \pm 0.25$ & 964.0 & 0.052 & $-0.762 \pm 0.003$ & $0.183 \pm 0.008$ \\
\hline NGC 1097 E & 024619.08 & $\begin{array}{llll}-30 & 16 & 20.20\end{array}$ & $9.88 \pm 0.10$ & $2.96 \pm 0.04$ & $1.90 \pm 0.07$ & 275.0 & 0.831 & $-0.733 \pm 0.010$ & $0.250 \pm 0.022$ \\
\hline NGC 1097 C & 024619.45 & -301633.70 & $12.16 \pm 0.10$ & $3.28 \pm 0.04$ & $2.35 \pm 0.07$ & 275.0 & 0.514 & $-0.774 \pm 0.008$ & $0.152 \pm 0.021$ \\
\hline NGC 1097 Enuc. 1 & 024623.90 & -301750.50 & $<0.69$ & $<0.13$ & $0.31 \pm 0.05$ & 688.0 & 7.301 & $\ldots$ & $\ldots$ \\
\hline NGC $1266 \mathrm{C}$ & 031600.76 & -022536.50 & $30.00 \pm 0.03$ & $8.94 \pm 0.02$ & $5.61 \pm 0.07$ & 445.0 & 0.390 & $-0.749 \pm 0.001$ & $0.214 \pm 0.003$ \\
\hline NGC $1266 \mathrm{~A}^{\mathrm{a}}$ & 031600.76 & $-02 \quad 2539.20$ & $35.62 \pm 0.08$ & $6.89 \pm 0.04$ & $3.99 \pm 0.16$ & 1038.0 & 0.267 & $-1.015 \pm 0.004$ & $0.234 \pm 0.008$ \\
\hline NGC 1266 B & 031600.76 & -022542.50 & $20.53 \pm 0.03$ & $4.63 \pm 0.02$ & $2.43 \pm 0.07$ & 445.0 & 0.964 & $-0.924 \pm 0.003$ & $0.239 \pm 0.006$ \\
\hline NGC 1377 & 033639.09 & -205406.60 & $0.20 \pm 0.02$ & $0.07 \pm 0.02$ & $0.06 \pm 0.02$ & 358.0 & 0.344 & $-0.665 \pm 0.141$ & $0.396 \pm 0.275$ \\
\hline IC 342 B & 034647.80 & +680546.30 & $39.31 \pm 0.12$ & $18.40 \pm 0.03$ & $13.99 \pm 0.08$ & 64.0 & 0.065 & $-0.458 \pm 0.002$ & $0.715 \pm 0.002$ \\
\hline IC $342 \mathrm{~A}^{\mathrm{a}}$ & 034648.45 & +680546.30 & $90.08 \pm 0.30$ & $36.82 \pm 0.08$ & $27.26 \pm 0.20$ & 159.0 & 0.007 & $-0.539 \pm 0.002$ & $0.608 \pm 0.003$ \\
\hline IC $342 \mathrm{C}$ & 034649.14 & +680548.40 & $23.28 \pm 0.12$ & $10.38 \pm 0.03$ & $8.02 \pm 0.08$ & 64.0 & 0.074 & $-0.482 \pm 0.003$ & $0.685 \pm 0.004$ \\
\hline IC $342 \mathrm{D}$ & 034650.918 & +680605.89 & $0.90 \pm 0.12$ & $0.13 \pm 0.04$ & $<0.30$ & 64.0 & 0.404 & $-1.178 \pm 0.184$ & $0.227 \pm 0.368$ \\
\hline NGC $1482 \mathrm{C}$ & 035438.73 & -203007.30 & $46.27 \pm 0.03$ & $12.80 \pm 0.02$ & $9.01 \pm 0.15$ & 438.0 & 0.272 & $-0.795 \pm 0.001$ & $0.097 \pm 0.003$ \\
\hline NGC $1482 A^{a}$ & 035438.97 & -203008.20 & $117.13 \pm 0.09$ & $24.25 \pm 0.06$ & $11.30 \pm 0.40$ & 1205.0 & 0.104 & $-0.978 \pm 0.002$ & $0.236 \pm 0.004$ \\
\hline NGC 1482 B & 035439.22 & -203008.20 & $43.02 \pm 0.03$ & $12.63 \pm 0.02$ & $8.71 \pm 0.14$ & 438.0 & 0.516 & $-0.759 \pm 0.001$ & $0.190 \pm 0.003$ \\
\hline NGC 2146 A & 061831.67 & +782143.84 & $6.15 \pm 0.14$ & $0.44 \pm 0.06$ & $2.29 \pm 0.12$ & 417.0 & 3.862 & $-0.496 \pm 0.024$ & $0.667 \pm 0.031$ \\
\hline NGC 2146 B & 061833.90 & +78 2134.94 & $18.19 \pm 0.08$ & $1.15 \pm 0.03$ & $5.80 \pm 0.06$ & 250.0 & 2.205 & $-0.542 \pm 0.005$ & $0.604 \pm 0.007$ \\
\hline NGC $2146 \mathrm{C}$ & 061835.34 & +782130.75 & $36.86 \pm 0.08$ & $1.68 \pm 0.03$ & $9.97 \pm 0.06$ & 250.0 & 1.322 & $-0.594 \pm 0.002$ & $0.523 \pm 0.004$ \\
\hline NGC 2146 D & 061838.35 & +78 2123.02 & $248.45 \pm 0.22$ & $21.18 \pm 0.08$ & $61.74 \pm 0.15$ & 667.0 & 0.410 & $-0.700 \pm 0.001$ & $0.324 \pm 0.002$ \\
\hline NGC 2146 E & 061843.83 & +78 2111.70 & $13.61 \pm 0.11$ & $0.58 \pm 0.04$ & $3.59 \pm 0.09$ & 334.0 & 3.245 & $-0.619 \pm 0.011$ & $0.480 \pm 0.019$ \\
\hline NGC 2403 Enuc. 5 C & 073619.35 & +653706.40 & $0.93 \pm 0.03$ & $0.75 \pm 0.02$ & $0.74 \pm 0.02$ & 62.0 & 3.520 & $-0.101 \pm 0.016$ & $0.999 \pm 0.008$ \\
\hline NGC 2403 Enuc. $5 \mathrm{~A}^{\mathrm{a}}$ & 073619.84 & +653706.70 & $2.05 \pm 0.06$ & $2.09 \pm 0.04$ & $1.83 \pm 0.05$ & 140.0 & 3.458 & $-0.038 \pm 0.016$ & $1.000 \pm 0.007$ \\
\hline NGC 2403 Enuc. 5 E & 073620.696 & +65 3804.84 & $0.24 \pm 0.03$ & $0.24 \pm 0.03$ & $<0.47$ & 62.0 & 3.428 & $0.011 \pm 0.098$ & $1.000 \pm 0.038$ \\
\hline
\end{tabular}


Table 4

\begin{tabular}{|c|c|c|c|c|c|c|c|c|c|}
\hline Source ID & $\begin{array}{l}\text { R.A. } \\
\text { (J2000) }\end{array}$ & $\begin{array}{l}\text { Decl. } \\
\text { (J2000) }\end{array}$ & $\begin{array}{c}S_{3 \mathrm{GHz}} \\
(\mathrm{mJy})\end{array}$ & $\begin{array}{c}S_{15 \mathrm{GHz}} \\
(\mathrm{mJy})\end{array}$ & $\begin{array}{c}S_{33 \mathrm{GHz}} \\
(\mathrm{mJy})\end{array}$ & $\begin{array}{l}d_{\mathrm{ap}} \\
(\mathrm{pc})\end{array}$ & $\begin{array}{c}r_{\mathrm{G}} \\
(\mathrm{kpc})\end{array}$ & $\alpha$ & $f_{\mathrm{T}}^{33 \mathrm{GHz}}$ \\
\hline NGC 2403 Enuc. 5 B & 073622.99 & +653649.90 & $0.17 \pm 0.03$ & $0.17 \pm 0.02$ & $0.10 \pm 0.03$ & 62.0 & 3.150 & $-0.109 \pm 0.104$ & $0.995 \pm 0.053$ \\
\hline NGC 2403 Enuc. 6 A & 073628.69 & +653349.95 & $0.87 \pm 0.05$ & $0.87 \pm 0.04$ & $0.78 \pm 0.03$ & 125.0 & 5.369 & $-0.048 \pm 0.029$ & $1.000 \pm 0.013$ \\
\hline NGC 2403 Enuc. 6 B & 073629.56 & +653405.30 & $0.12 \pm 0.03$ & $<0.06$ & $0.05 \pm 0.02$ & 62.0 & 4.933 & $-0.363 \pm 0.165$ & $0.815 \pm 0.156$ \\
\hline NGC 2403 Enuc. $1 \mathrm{E}$ & 073638.042 & +653609.31 & $0.24 \pm 0.03$ & $0.29 \pm 0.03$ & $<0.96$ & 62.0 & 1.529 & $0.110 \pm 0.098$ & $1.000 \pm 0.030$ \\
\hline NGC 2403 Enuc. 1 A & 073642.03 & +65 3651.67 & $0.51 \pm 0.03$ & $0.39 \pm 0.02$ & $0.25 \pm 0.03$ & 62.0 & 1.085 & $-0.221 \pm 0.040$ & $0.929 \pm 0.027$ \\
\hline NGC $2403 \mathrm{C}$ & 073642.562 & +653607.23 & $0.13 \pm 0.03$ & $0.14 \pm 0.03$ & $<0.14$ & 62.0 & 0.952 & $0.038 \pm 0.186$ & $1.000 \pm 0.067$ \\
\hline NGC 2403 Enuc. 1 B & 073645.49 & +653701.39 & $1.52 \pm 0.06$ & $1.37 \pm 0.04$ & $1.14 \pm 0.04$ & 125.0 & 1.203 & $-0.106 \pm 0.021$ & $0.997 \pm 0.011$ \\
\hline NGC 2403 Enuc. $1 \mathrm{C}$ & 073645.74 & +653639.90 & $0.47 \pm 0.03$ & $0.10 \pm 0.02$ & $0.10 \pm 0.03$ & 62.0 & 0.753 & $-0.799 \pm 0.093$ & $0.088 \pm 0.249$ \\
\hline NGC 2403 Enuc. 1 D & 073646.71 & +653637.50 & $0.20 \pm 0.03$ & $0.13 \pm 0.02$ & $0.11 \pm 0.03$ & 62.0 & 0.697 & $-0.257 \pm 0.103$ & $0.904 \pm 0.075$ \\
\hline NGC 2403 Enuc. 2 B & 073649.15 & +653651.72 & $0.42 \pm 0.04$ & $0.32 \pm 0.03$ & $0.28 \pm 0.04$ & 94.0 & 1.115 & $-0.167 \pm 0.067$ & $0.963 \pm 0.040$ \\
\hline NGC 2403 Enuc. 2 D & 073652.07 & +653648.40 & $0.64 \pm 0.02$ & $0.55 \pm 0.02$ & $0.56 \pm 0.02$ & 47.0 & 1.258 & $-0.060 \pm 0.018$ & $1.000 \pm 0.008$ \\
\hline NGC 2403 Enuc. $2 \mathrm{~A}^{\mathrm{a}}$ & 073652.46 & +653645.70 & $2.14 \pm 0.06$ & $1.53 \pm 0.04$ & $1.48 \pm 0.04$ & 125.0 & 1.229 & $-0.166 \pm 0.016$ & $0.964 \pm 0.009$ \\
\hline NGC 2403 Enuc. 2 C & 073652.80 & +653645.10 & $0.60 \pm 0.02$ & $0.45 \pm 0.02$ & $0.40 \pm 0.02$ & 47.0 & 1.247 & $-0.170 \pm 0.021$ & $0.961 \pm 0.013$ \\
\hline NGC 2403 B & 073655.91 & +653538.49 & $0.19 \pm 0.03$ & $<0.08$ & $<0.13$ & 62.0 & 0.699 & $\ldots$ & $\ldots$ \\
\hline NGC 2403 E & 073656.727 & +653512.17 & $0.14 \pm 0.03$ & $<0.10$ & $<0.58$ & 62.0 & 1.101 & $\cdots$ & $\ldots$ \\
\hline NGC 2403 Enuc. 3 B & 073706.66 & +653638.40 & $3.81 \pm 0.04$ & $3.15 \pm 0.03$ & $2.89 \pm 0.04$ & 78.0 & 2.763 & $-0.116 \pm 0.006$ & $0.992 \pm 0.003$ \\
\hline NGC 2403 Enuc. 4 B & 073716.55 & +653350.80 & $<0.08$ & $0.12 \pm 0.02$ & $0.07 \pm 0.02$ & 62.0 & 3.305 & $-0.560 \pm 0.358$ & $0.577 \pm 0.544$ \\
\hline NGC 2403 Enuc. 4 C & 073718.14 & +653337.90 & $<0.08$ & $0.11 \pm 0.02$ & $0.08 \pm 0.02$ & 62.0 & 3.556 & $-0.368 \pm 0.359$ & $0.810 \pm 0.345$ \\
\hline NGC 2403 Enuc. 4 A & 073718.30 & +653349.90 & $1.00 \pm 0.06$ & $0.99 \pm 0.04$ & $0.92 \pm 0.03$ & 125.0 & 3.446 & $-0.037 \pm 0.028$ & $1.000 \pm 0.012$ \\
\hline Holmberg II B & 081912.69 & +704303.80 & $0.15 \pm 0.02$ & $0.14 \pm 0.01$ & $0.17 \pm 0.02$ & 44.0 & 0.721 & $0.018 \pm 0.078$ & $1.000 \pm 0.030$ \\
\hline Holmberg II A & 081913.09 & +704308.90 & $0.33 \pm 0.03$ & $0.31 \pm 0.02$ & $0.34 \pm 0.03$ & 59.0 & 0.739 & $-0.003 \pm 0.048$ & $1.000 \pm 0.019$ \\
\hline NGC $2798 \mathrm{~B}$ & 091722.56 & +415951.10 & $1.76 \pm 0.02$ & $0.61 \pm 0.02$ & $0.19 \pm 0.03$ & 375.0 & 2.580 & $-0.693 \pm 0.022$ & $0.338 \pm 0.045$ \\
\hline NGC $2798 \mathrm{~A}$ & 091722.83 & +420001.00 & $11.23 \pm 0.06$ & $2.53 \pm 0.06$ & $0.93 \pm 0.07$ & 1001.0 & 0.217 & $-0.945 \pm 0.013$ & $0.238 \pm 0.027$ \\
\hline NGC $2841 \mathrm{~A}$ & 092202.68 & +505835.74 & $0.64 \pm 0.04$ & $<0.18$ & $<0.08$ & 410.0 & 0.171 & $\ldots$ & $\ldots$ \\
\hline NGC 2976 Enuc. 1 A & 094705.09 & +675551.70 & $1.14 \pm 0.03$ & $0.76 \pm 0.02$ & $0.75 \pm 0.05$ & 86.0 & 1.432 & $-0.219 \pm 0.020$ & $0.930 \pm 0.014$ \\
\hline NGC 2976 Enuc. 1 D & 094707.64 & +675555.30 & $2.22 \pm 0.02$ & $1.80 \pm 0.02$ & $1.56 \pm 0.03$ & 69.0 & 1.208 & $-0.136 \pm 0.008$ & $0.981 \pm 0.004$ \\
\hline NGC 2976 Enuc. 1 C & 094707.85 & +675548.10 & $0.48 \pm 0.02$ & $0.37 \pm 0.01$ & $0.33 \pm 0.02$ & 52.0 & 1.111 & $-0.162 \pm 0.027$ & $0.966 \pm 0.016$ \\
\hline NGC 2976 Enuc. $1 \mathrm{~B}^{\mathrm{a}}$ & 094707.85 & +67 5553.98 & $3.26 \pm 0.06$ & $2.49 \pm 0.05$ & $2.01 \pm 0.08$ & 172.0 & 1.178 & $-0.182 \pm 0.014$ & $0.954 \pm 0.008$ \\
\hline NGC 2976 B & 094713.54 & +675454.90 & $0.17 \pm 0.02$ & $0.16 \pm 0.02$ & $0.17 \pm 0.03$ & 69.0 & 0.432 & $-0.017 \pm 0.090$ & $1.000 \pm 0.037$ \\
\hline NGC 2976 A & 094715.30 & +675500.00 & $<0.07$ & $0.15 \pm 0.02$ & $<0.10$ & 69.0 & 0.000 & $\ldots$ & $\ldots$ \\
\hline NGC 2976 Enuc. 2 A & 094723.94 & +675353.40 & $1.67 \pm 0.04$ & $1.46 \pm 0.03$ & $1.25 \pm 0.06$ & 120.0 & 1.421 & $-0.098 \pm 0.018$ & $1.000 \pm 0.009$ \\
\hline NGC 2976 Enuc. 2 C & 094723.94 & +675405.40 & $0.55 \pm 0.04$ & $0.45 \pm 0.03$ & $0.40 \pm 0.05$ & 103.0 & 1.278 & $-0.131 \pm 0.047$ & $0.984 \pm 0.026$ \\
\hline NGC 2976 C & 094725.079 & +67 5509.45 & $0.25 \pm 0.02$ & $<0.07$ & $<0.48$ & 69.0 & 2.002 & $\ldots$ & $\ldots$ \\
\hline NGC 3049 A & 095449.56 & +09 1616.05 & $1.47 \pm 0.04$ & $0.53 \pm 0.04$ & $0.47 \pm 0.04$ & 559.0 & 0.110 & $-0.546 \pm 0.032$ & $0.598 \pm 0.048$ \\
\hline NGC 3049 B & 095450.05 & +091625.40 & $0.32 \pm 0.03$ & $0.22 \pm 0.02$ & $0.16 \pm 0.03$ & 372.0 & 1.033 & $-0.272 \pm 0.070$ & $0.893 \pm 0.053$ \\
\hline NGC $3049 \mathrm{C}$ & 095451.46 & +091628.40 & $0.21 \pm 0.02$ & $<0.06$ & $<0.10$ & 279.0 & 3.622 & $\ldots$ & $\ldots$ \\
\hline NGC 3077 A & 100319.15 & +684359.90 & $11.35 \pm 0.06$ & $7.21 \pm 0.05$ & $6.19 \pm 0.12$ & 186.0 & 0.045 & $-0.273 \pm 0.005$ & $0.892 \pm 0.004$ \\
\hline NGC $3190 \mathrm{~A}$ & 101804.31 & +214931.79 & $0.56 \pm 0.03$ & $0.61 \pm 0.02$ & $1.17 \pm 0.04$ & 374.0 & 9.635 & $0.315 \pm 0.024$ & $1.000 \pm 0.005$ \\
\hline NGC $3184 \mathrm{~B}$ & 101811.442 & +412449.70 & $0.33 \pm 0.04$ & $0.20 \pm 0.04$ & $<1.56$ & 340.0 & 4.382 & $-0.323 \pm 0.153$ & $0.851 \pm 0.132$ \\
\hline NGC $3184 \mathrm{~A}$ & 101816.91 & +412527.02 & $0.65 \pm 0.04$ & $0.33 \pm 0.03$ & $0.30 \pm 0.03$ & 340.0 & 0.057 & $-0.363 \pm 0.044$ & $0.815 \pm 0.042$ \\
\hline NGC $3198 \mathrm{~B}$ & 101950.990 & +453250.16 & $0.21 \pm 0.04$ & $0.14 \pm 0.03$ & $<0.34$ & 410.0 & 5.622 & $-0.233 \pm 0.193$ & $0.921 \pm 0.134$ \\
\hline NGC $3198 \mathrm{~A}$ & 101955.02 & +453259.24 & $0.49 \pm 0.04$ & $0.28 \pm 0.03$ & $0.19 \pm 0.05$ & 410.0 & 0.180 & $-0.354 \pm 0.074$ & $0.824 \pm 0.068$ \\
\hline NGC $3198 \mathrm{C}$ & 101957.979 & +453228.54 & $0.17 \pm 0.04$ & $0.17 \pm 0.03$ & $<0.39$ & 410.0 & 8.035 & $0.021 \pm 0.201$ & $1.000 \pm 0.076$ \\
\hline IC 2574 D & 102840.72 & +682801.39 & $0.98 \pm 0.03$ & $0.31 \pm 0.02$ & $0.48 \pm 0.08$ & 92.0 & 5.606 & $-0.596 \pm 0.040$ & $0.520 \pm 0.066$ \\
\hline IC $2574 \mathrm{C}$ & 102843.88 & +682826.90 & $0.90 \pm 0.04$ & $0.63 \pm 0.03$ & $0.77 \pm 0.08$ & 129.0 & 6.262 & $-0.164 \pm 0.034$ & $0.965 \pm 0.020$ \\
\hline IC $2574 \mathrm{~A}$ & 102848.41 & +682803.28 & $0.60 \pm 0.03$ & $0.50 \pm 0.02$ & $0.48 \pm 0.03$ & 92.0 & 5.247 & $-0.105 \pm 0.031$ & $0.997 \pm 0.016$ \\
\hline IC 2574 B & 102848.78 & +682835.90 & $0.08 \pm 0.02$ & $0.11 \pm 0.02$ & $<0.13$ & 73.0 & 6.263 & $0.157 \pm 0.195$ & $1.000 \pm 0.053$ \\
\hline
\end{tabular}


Table 4

(Continued)

\begin{tabular}{|c|c|c|c|c|c|c|c|c|c|}
\hline Source ID & $\begin{array}{l}\text { R.A. } \\
\text { (J2000) }\end{array}$ & $\begin{array}{l}\text { Decl. } \\
\text { (J2000) }\end{array}$ & $\begin{array}{l}S_{3 \mathrm{GHz}} \\
(\mathrm{mJy})\end{array}$ & $\begin{array}{c}S_{15 \mathrm{GHz}} \\
(\mathrm{mJy})\end{array}$ & $\begin{array}{c}S_{33 \mathrm{GHz}} \\
(\mathrm{mJy})\end{array}$ & $\begin{array}{l}d_{\mathrm{ap}} \\
(\mathrm{pc})\end{array}$ & $\begin{array}{c}r_{\mathrm{G}} \\
(\mathrm{kpc})\end{array}$ & $\alpha$ & $f_{\mathrm{T}}^{33 \mathrm{GHz}}$ \\
\hline NGC $3265 \mathrm{~A}$ & 103106.77 & +284748.01 & $2.76 \pm 0.05$ & $0.67 \pm 0.03$ & $0.43 \pm 0.04$ & 665.0 & 0.090 & $-0.849 \pm 0.024$ & $0.245 \pm 0.053$ \\
\hline NGC $3351 \mathrm{C}$ & 104355.25 & +114129.90 & $2.20 \pm 0.04$ & $1.04 \pm 0.04$ & $1.21 \pm 0.19$ & 181.0 & 2.844 & $-0.439 \pm 0.024$ & $0.737 \pm 0.028$ \\
\hline NGC 3351 A & 104357.67 & +114207.19 & $7.20 \pm 0.06$ & $2.75 \pm 0.04$ & $1.95 \pm 0.05$ & 271.0 & 0.267 & $-0.572 \pm 0.009$ & $0.558 \pm 0.013$ \\
\hline NGC 3351 B & 104357.77 & +114218.88 & $8.84 \pm 0.06$ & $3.55 \pm 0.04$ & $2.26 \pm 0.05$ & 271.0 & 0.271 & $-0.567 \pm 0.007$ & $0.566 \pm 0.011$ \\
\hline NGC 3521 Enuc. 1 & 110546.36 & -000410.10 & $0.14 \pm 0.04$ & $0.29 \pm 0.04$ & $<0.15$ & 271.0 & 9.918 & $0.469 \pm 0.194$ & $1.000 \pm 0.025$ \\
\hline NGC 3521 Enuc. 3 A & 110547.64 & +000032.80 & $<0.20$ & $0.31 \pm 0.05$ & $<0.18$ & 489.0 & 9.517 & $\ldots$ & $\ldots$ \\
\hline NGC 3521 Enuc. $2 \mathrm{~A}$ & 110549.28 & -000326.70 & $0.66 \pm 0.04$ & $0.35 \pm 0.04$ & $0.28 \pm 0.05$ & 271.0 & 4.474 & $-0.377 \pm 0.058$ & $0.802 \pm 0.056$ \\
\hline NGC 3521 Enuc. $3 \mathrm{C}$ & 110549.421 & -00029.52 & $0.99 \pm 0.07$ & $<0.22$ & $<2.62$ & 489.0 & 6.970 & $\ldots$ & $\ldots$ \\
\hline NGC 3521 Enuc. 2 B & 110551.04 & -000349.20 & $0.30 \pm 0.04$ & $0.36 \pm 0.04$ & $<0.15$ & 271.0 & 5.824 & $0.121 \pm 0.104$ & $1.000 \pm 0.031$ \\
\hline NGC $3621 \mathrm{C}$ & 111814.878 & -324735.40 & $0.38 \pm 0.04$ & $0.32 \pm 0.04$ & $<2.10$ & 191.0 & 2.191 & $-0.109 \pm 0.105$ & $0.995 \pm 0.054$ \\
\hline NGC $3621 \mathrm{~A}$ & 111815.21 & -324839.60 & $1.05 \pm 0.04$ & $0.27 \pm 0.03$ & $<0.16$ & 191.0 & 0.841 & $-0.851 \pm 0.071$ & $0.245 \pm 0.154$ \\
\hline NGC $3621 \mathrm{H}$ & 111816.465 & -324856.84 & $0.11 \pm 0.04$ & $<0.09$ & $<0.17$ & 191.0 & 0.574 & $\ldots$ & $\ldots$ \\
\hline NGC $3621 \mathrm{G}^{\mathrm{a}}$ & 111818.117 & -324939.41 & $2.15 \pm 0.07$ & $1.55 \pm 0.08$ & $<2.71$ & 381.0 & 1.967 & $-0.202 \pm 0.040$ & $0.942 \pm 0.025$ \\
\hline NGC 3621 B & 111818.936 & -324744.04 & $0.93 \pm 0.05$ & $0.56 \pm 0.06$ & $<2.97$ & 254.0 & 3.029 & $-0.321 \pm 0.074$ & $0.853 \pm 0.064$ \\
\hline NGC $3621 \mathrm{~F}$ & 111820.832 & -324853.41 & $0.14 \pm 0.04$ & $<0.12$ & $<1.22$ & 191.0 & 2.760 & $\ldots$ & $\ldots$ \\
\hline NGC $3621 \mathrm{E}$ & 111821.542 & -324908.39 & $0.62 \pm 0.04$ & $0.19 \pm 0.05$ & $<4.80$ & 191.0 & 3.143 & $-0.722 \pm 0.162$ & $0.277 \pm 0.362$ \\
\hline NGC 3627 Enuc. $1 \mathrm{G}$ & 112011.500 & +125755.42 & $0.52 \pm 0.06$ & $<0.33$ & $<2.45$ & 318.0 & 7.592 & $\ldots$ & $\ldots$ \\
\hline NGC 3627 Enuc. $1 \mathrm{~F}$ & 112012.848 & +125756.98 & $0.24 \pm 0.03$ & $<0.15$ & $<0.32$ & 182.0 & 5.975 & $\ldots$ & $\ldots$ \\
\hline NGC 3627 Enuc. $1 \mathrm{E}$ & 112012.989 & +125736.49 & $0.30 \pm 0.03$ & $<0.15$ & $0.40 \pm 0.10$ & 182.0 & 6.672 & $0.124 \pm 0.116$ & $1.000 \pm 0.034$ \\
\hline NGC 3627 B & 112013.34 & +130021.30 & $3.99 \pm 0.07$ & $2.42 \pm 0.10$ & $1.46 \pm 0.37$ & 364.0 & 3.072 & $-0.319 \pm 0.028$ & $0.855 \pm 0.024$ \\
\hline NGC 3627 A & 112015.01 & +125929.53 & $1.95 \pm 0.05$ & $1.10 \pm 0.06$ & $0.69 \pm 0.05$ & 273.0 & 0.023 & $-0.397 \pm 0.026$ & $0.781 \pm 0.027$ \\
\hline NGC 3627 Enuc. 2 D & 112016.26 & +125844.30 & $4.11 \pm 0.03$ & $2.49 \pm 0.04$ & $1.90 \pm 0.02$ & 136.0 & 2.534 & $-0.318 \pm 0.005$ & $0.855 \pm 0.004$ \\
\hline NGC 3627 Enuc. $1 \mathrm{~A}$ & 112016.33 & +125749.39 & $2.42 \pm 0.05$ & $1.44 \pm 0.06$ & $1.26 \pm 0.05$ & 273.0 & 4.706 & $-0.282 \pm 0.017$ & $0.885 \pm 0.013$ \\
\hline NGC 3627 Enuc. $2 \mathrm{~A}^{\mathrm{a}}$ & 112016.53 & +125844.30 & $7.18 \pm 0.09$ & $4.22 \pm 0.12$ & $3.51 \pm 0.08$ & 455.0 & 2.781 & $-0.304 \pm 0.010$ & $0.868 \pm 0.008$ \\
\hline NGC 3627 Enuc. 2 E & 112016.71 & +125843.40 & $4.13 \pm 0.03$ & $2.10 \pm 0.04$ & $1.67 \pm 0.02$ & 136.0 & 2.994 & $-0.385 \pm 0.006$ & $0.793 \pm 0.006$ \\
\hline NGC 3627 Enuc. $2 \mathrm{C}$ & 112017.98 & +125906.20 & $0.43 \pm 0.03$ & $0.43 \pm 0.05$ & $0.24 \pm 0.05$ & 182.0 & 4.390 & $-0.124 \pm 0.072$ & $0.987 \pm 0.038$ \\
\hline NGC 3627 Enuc. 2 B & 112018.70 & +125924.20 & $0.77 \pm 0.03$ & $0.51 \pm 0.06$ & $<0.38$ & 182.0 & 5.526 & $-0.259 \pm 0.081$ & $0.903 \pm 0.060$ \\
\hline NGC 3773 & 113813.02 & +120644.10 & $2.53 \pm 0.04$ & $1.34 \pm 0.04$ & $1.09 \pm 0.05$ & 481.0 & 0.023 & $-0.372 \pm 0.016$ & $0.806 \pm 0.016$ \\
\hline NGC 3938 B & 115246.860 & +440807.38 & $0.28 \pm 0.03$ & $<0.08$ & $<0.59$ & 434.0 & 5.503 & $\ldots$ & $\ldots$ \\
\hline NGC 3938 Enuc. 2 B & 115259.97 & +440800.00 & $0.19 \pm 0.02$ & $0.13 \pm 0.02$ & $0.18 \pm 0.03$ & 347.0 & 11.079 & $-0.069 \pm 0.080$ & $1.000 \pm 0.037$ \\
\hline NGC 3938 Enuc. 2 A & 115300.20 & +440748.07 & $0.34 \pm 0.03$ & $0.28 \pm 0.02$ & $0.23 \pm 0.04$ & 434.0 & 11.048 & $-0.139 \pm 0.061$ & $0.979 \pm 0.034$ \\
\hline NGC 4254 Enuc. 2 D & 121845.82 & +142409.80 & $<0.09$ & $0.10 \pm 0.03$ & $0.17 \pm 0.02$ & 279.0 & 5.332 & $0.670 \pm 0.371$ & $1.000 \pm 0.029$ \\
\hline NGC 4254 Enuc. $2 \mathrm{C}$ & 121846.19 & +142411.90 & $<0.07$ & $<0.06$ & $0.08 \pm 0.02$ & 209.0 & 4.931 & $\ldots$ & $\ldots$ \\
\hline NGC 4254 Enuc. $1 \mathrm{E}$ & 121846.77 & +142351.00 & $0.29 \pm 0.03$ & $0.11 \pm 0.02$ & $<0.14$ & 279.0 & 5.622 & $-0.602 \pm 0.132$ & $0.509 \pm 0.223$ \\
\hline NGC 4254 Enuc. 2 E & 121847.495 & +142441.83 & $0.14 \pm 0.03$ & $<0.09$ & $<0.14$ & 279.0 & 2.617 & $\ldots$ & $\ldots$ \\
\hline NGC 4254 & 121849.54 & +142501.40 & $0.41 \pm 0.06$ & $0.52 \pm 0.04$ & $0.36 \pm 0.05$ & 559.0 & 0.124 & $-0.008 \pm 0.085$ & $1.000 \pm 0.034$ \\
\hline NGC 4254 Enuc. $1 \mathrm{D}$ & 121850.03 & +142417.10 & $0.79 \pm 0.04$ & $0.23 \pm 0.02$ & $0.35 \pm 0.04$ & 349.0 & 3.167 & $-0.487 \pm 0.047$ & $0.678 \pm 0.060$ \\
\hline NGC 4254 Enuc. 1 B & 121850.07 & +142406.60 & $0.21 \pm 0.05$ & $0.12 \pm 0.03$ & $0.41 \pm 0.05$ & 419.0 & 3.924 & $0.321 \pm 0.100$ & $1.000 \pm 0.018$ \\
\hline NGC 4254 Enuc. $1 \mathrm{C}$ & 121850.44 & +142422.50 & $0.21 \pm 0.02$ & $<0.05$ & $0.13 \pm 0.03$ & 209.0 & 2.953 & $-0.204 \pm 0.110$ & $0.941 \pm 0.071$ \\
\hline NGC 4321 Enuc. 2 & 122249.90 & +155028.70 & $0.17 \pm 0.04$ & $<0.08$ & $0.11 \pm 0.03$ & 347.0 & 8.026 & $-0.176 \pm 0.132$ & $0.958 \pm 0.080$ \\
\hline NGC $4321 \mathrm{C}$ & 122254.46 & +154921.30 & $2.57 \pm 0.03$ & $1.09 \pm 0.02$ & $0.59 \pm 0.03$ & 277.0 & 0.500 & $-0.560 \pm 0.014$ & $0.577 \pm 0.021$ \\
\hline NGC $4321 \mathrm{~F}$ & 122254.63 & +154914.70 & $0.93 \pm 0.02$ & $0.45 \pm 0.02$ & $0.31 \pm 0.02$ & 208.0 & 0.514 & $-0.451 \pm 0.024$ & $0.723 \pm 0.028$ \\
\hline NGC $4321 \mathrm{H}$ & 122254.92 & +154909.90 & $0.65 \pm 0.02$ & $0.31 \pm 0.02$ & $0.27 \pm 0.02$ & 208.0 & 0.810 & $-0.401 \pm 0.032$ & $0.778 \pm 0.033$ \\
\hline NGC $4321 A^{a}$ & 122254.92 & +154921.00 & $23.09 \pm 0.12$ & $8.99 \pm 0.09$ & $5.06 \pm 0.11$ & 1040.0 & 0.024 & $-0.603 \pm 0.006$ & $0.508 \pm 0.010$ \\
\hline NGC 4321 B & 122254.94 & +154920.40 & $2.47 \pm 0.02$ & $0.79 \pm 0.02$ & $0.45 \pm 0.02$ & 208.0 & 0.069 & $-0.707 \pm 0.013$ & $0.308 \pm 0.028$ \\
\hline NGC $4321 \mathrm{G}$ & 122254.94 & +154929.40 & $1.77 \pm 0.03$ & $0.63 \pm 0.02$ & $0.38 \pm 0.03$ & 277.0 & 0.605 & $-0.644 \pm 0.022$ & $0.435 \pm 0.041$ \\
\hline NGC $4321 \mathrm{D}$ & 122255.29 & +154915.30 & $3.26 \pm 0.03$ & $1.27 \pm 0.02$ & $0.83 \pm 0.03$ & 277.0 & 0.653 & $-0.578 \pm 0.011$ & $0.549 \pm 0.017$ \\
\hline
\end{tabular}


Table 4

(Continued)

\begin{tabular}{|c|c|c|c|c|c|c|c|c|c|}
\hline Source ID & $\begin{array}{l}\text { R.A. } \\
\text { (J2000) }\end{array}$ & $\begin{array}{l}\text { Decl. } \\
\text { (J2000) }\end{array}$ & $\begin{array}{l}S_{3 \mathrm{GHz}} \\
(\mathrm{mJy})\end{array}$ & $\begin{array}{c}S_{15 \mathrm{GHz}} \\
(\mathrm{mJy})\end{array}$ & $\begin{array}{c}S_{33 \mathrm{GHz}} \\
(\mathrm{mJy})\end{array}$ & $\begin{array}{l}d_{\mathrm{ap}} \\
(\mathrm{pc})\end{array}$ & $\begin{array}{c}r_{\mathrm{G}} \\
(\mathrm{kpc})\end{array}$ & $\alpha$ & $f_{\mathrm{T}}^{33 \mathrm{GHz}}$ \\
\hline NGC $4321 \mathrm{E}$ & 122255.38 & +154923.40 & $3.94 \pm 0.03$ & $1.39 \pm 0.02$ & $0.87 \pm 0.03$ & 277.0 & 0.547 & $-0.641 \pm 0.010$ & $\overline{0.442 \pm 0.018}$ \\
\hline NGC 4321 Enuc. $1 \mathrm{~B}$ & 122258.82 & +154850.60 & $0.28 \pm 0.02$ & $0.17 \pm 0.02$ & $<0.12$ & 208.0 & 5.246 & $-0.321 \pm 0.094$ & $0.853 \pm 0.081$ \\
\hline NGC $4536 \mathrm{C}$ & 123426.94 & +021119.40 & $33.72 \pm 0.03$ & $12.67 \pm 0.02$ & $8.01 \pm 0.03$ & 281.0 & 0.243 & $-0.605 \pm 0.001$ & $0.504 \pm 0.002$ \\
\hline NGC $4536 \mathrm{~A}^{\mathrm{a}}$ & 123427.08 & +021117.30 & $88.63 \pm 0.10$ & $28.11 \pm 0.06$ & $16.57 \pm 0.09$ & 844.0 & 0.030 & $-0.710 \pm 0.001$ & $0.303 \pm 0.003$ \\
\hline NGC 4536 B & 123427.34 & +021115.50 & $27.18 \pm 0.03$ & $9.90 \pm 0.02$ & $6.51 \pm 0.03$ & 281.0 & 0.330 & $-0.617 \pm 0.001$ & $0.483 \pm 0.002$ \\
\hline NGC $4559 \mathrm{E}$ & 123555.427 & +275801.88 & $0.15 \pm 0.04$ & $0.12 \pm 0.04$ & $<0.15$ & 203.0 & 1.703 & $-0.106 \pm 0.245$ & $0.997 \pm 0.125$ \\
\hline NGC $4559 \mathrm{C}$ & 123556.27 & +275740.80 & $0.20 \pm 0.04$ & $0.18 \pm 0.03$ & $0.21 \pm 0.03$ & 203.0 & 1.294 & $0.014 \pm 0.100$ & $1.000 \pm 0.038$ \\
\hline NGC 4559 D & 123557.59 & +275736.90 & $<0.12$ & $0.12 \pm 0.03$ & $<0.07$ & 203.0 & 0.090 & $\ldots$ & $\ldots$ \\
\hline NGC 4559 B & 123558.36 & +275733.90 & $0.09 \pm 0.03$ & $0.16 \pm 0.02$ & $0.13 \pm 0.02$ & 135.0 & 0.599 & $0.088 \pm 0.123$ & $1.000 \pm 0.040$ \\
\hline NGC 4559 A & 123558.45 & +275727.90 & $0.16 \pm 0.02$ & $0.10 \pm 0.02$ & $0.14 \pm 0.01$ & 102.0 & 0.563 & $-0.051 \pm 0.065$ & $1.000 \pm 0.029$ \\
\hline NGC 4569 A & 123649.84 & +130946.59 & $2.89 \pm 0.07$ & $1.44 \pm 0.07$ & $0.88 \pm 0.08$ & 430.0 & 0.051 & $-0.460 \pm 0.026$ & $0.711 \pm 0.031$ \\
\hline NGC 4579 & 123743.52 & +114905.50 & $2.04 \pm 0.13$ & $2.76 \pm 0.22$ & $0.60 \pm 0.18$ & 795.0 & 0.103 & $0.077 \pm 0.060$ & $1.000 \pm 0.020$ \\
\hline NGC 4594 & 123959.43 & -113723.03 & $0.86 \pm 0.09$ & $0.46 \pm 0.06$ & $0.83 \pm 0.08$ & 440.0 & 0.046 & $-0.048 \pm 0.061$ & $1.000 \pm 0.027$ \\
\hline NGC 4631 Enuc. 1 & 124140.47 & +323150.00 & $0.51 \pm 0.05$ & $0.66 \pm 0.04$ & $0.61 \pm 0.04$ & 296.0 & 13.669 & $0.058 \pm 0.050$ & $1.000 \pm 0.017$ \\
\hline NGC $4625 \mathrm{~A}$ & 124152.27 & +411625.50 & $<0.12$ & $0.11 \pm 0.03$ & $<0.06$ & 271.0 & 0.190 & $\ldots$ & $\ldots$ \\
\hline NGC $4631 \mathrm{~A}$ & 124203.59 & +323216.28 & $7.26 \pm 0.03$ & $2.46 \pm 0.04$ & $1.51 \pm 0.04$ & 148.0 & 3.389 & $-0.666 \pm 0.008$ & $0.393 \pm 0.015$ \\
\hline NGC $4631 \mathrm{H}$ & 124204.17 & +323216.60 & $0.98 \pm 0.03$ & $1.31 \pm 0.03$ & $0.85 \pm 0.04$ & 148.0 & 3.276 & $0.031 \pm 0.019$ & $1.000 \pm 0.007$ \\
\hline NGC 4631 B & 124204.38 & +323225.08 & $5.50 \pm 0.04$ & $3.32 \pm 0.04$ & $3.07 \pm 0.04$ & 185.0 & 1.712 & $-0.265 \pm 0.006$ & $0.898 \pm 0.004$ \\
\hline NGC $4631 \mathrm{G}$ & 124205.07 & +323212.40 & $0.91 \pm 0.04$ & $0.73 \pm 0.04$ & $0.62 \pm 0.04$ & 185.0 & 4.464 & $-0.155 \pm 0.029$ & $0.970 \pm 0.017$ \\
\hline NGC 4631 Enuc. 2 B & 124222.22 & +323246.33 & $1.56 \pm 0.07$ & $1.40 \pm 0.05$ & $1.39 \pm 0.06$ & 369.0 & 6.825 & $-0.052 \pm 0.026$ & $1.000 \pm 0.012$ \\
\hline NGC 4631 Enuc. $2 \mathrm{C}$ & 124223.985 & +323208.65 & $<0.11$ & $0.22 \pm 0.03$ & $<0.92$ & 185.0 & 12.785 & $\cdots$ & $\ldots$ \\
\hline NGC 4725 C & 125024.87 & +252922.80 & $0.22 \pm 0.02$ & $0.08 \pm 0.02$ & $<0.12$ & 173.0 & 2.693 & $-0.597 \pm 0.136$ & $0.518 \pm 0.226$ \\
\hline NGC 4725 A & 125026.57 & +253002.72 & $0.07 \pm 0.02$ & $0.17 \pm 0.01$ & $0.30 \pm 0.01$ & 173.0 & 0.034 & $0.641 \pm 0.078$ & $1.000 \pm 0.007$ \\
\hline NGC 4736 K & 125049.204 & +410733.20 & $0.61 \pm 0.05$ & $0.78 \pm 0.08$ & $<0.42$ & 136.0 & 1.102 & $0.154 \pm 0.078$ & $1.000 \pm 0.021$ \\
\hline NGC $4736 \mathrm{~J}$ & 125049.219 & +410723.09 & $1.01 \pm 0.05$ & $0.49 \pm 0.08$ & $<0.36$ & 136.0 & 1.017 & $-0.447 \pm 0.100$ & $0.727 \pm 0.116$ \\
\hline NGC 4736 I & 125049.604 & +410709.41 & $1.27 \pm 0.05$ & $0.65 \pm 0.07$ & $<0.29$ & 136.0 & 0.922 & $-0.416 \pm 0.073$ & $0.762 \pm 0.079$ \\
\hline NGC 4736 G & 125049.935 & +410641.41 & $0.27 \pm 0.05$ & $<0.24$ & $<0.43$ & 136.0 & 1.248 & $\ldots$ & $\ldots$ \\
\hline NGC 4736L & 125050.030 & +410750.00 & $0.56 \pm 0.05$ & $<0.24$ & $<0.48$ & 136.0 & 1.222 & $\cdots$ & $\cdots$ \\
\hline NGC $4736 \mathrm{H}$ & 125050.045 & +410653.23 & $1.28 \pm 0.05$ & $1.08 \pm 0.07$ & $0.35 \pm 0.10$ & 136.0 & 1.002 & $-0.159 \pm 0.045$ & $0.968 \pm 0.026$ \\
\hline NGC 4736 M & 125050.746 & +410738.18 & $0.16 \pm 0.05$ & $<0.21$ & $<0.25$ & 136.0 & 0.872 & $\cdots$ & $\cdots$ \\
\hline NGC $4736 \mathrm{~F}$ & 125051.201 & +410649.50 & $1.35 \pm 0.05$ & $0.89 \pm 0.07$ & $<0.23$ & 136.0 & 0.846 & $-0.262 \pm 0.053$ & $0.900 \pm 0.040$ \\
\hline NGC 4736N & 125051.776 & +410748.36 & $0.26 \pm 0.05$ & $1.07 \pm 0.07$ & $<0.27$ & 136.0 & 0.991 & $0.878 \pm 0.117$ & $1.000 \pm 0.006$ \\
\hline NGC 4736 A & 125053.050 & +410712.50 & $6.19 \pm 0.05$ & $2.13 \pm 0.06$ & $1.23 \pm 0.05$ & 136.0 & 0.020 & $-0.668 \pm 0.013$ & $0.389 \pm 0.025$ \\
\hline NGC 4736 O & 125053.190 & +410746.40 & $0.17 \pm 0.05$ & $0.44 \pm 0.07$ & $0.38 \pm 0.08$ & 136.0 & 0.914 & $0.345 \pm 0.137$ & $1.000 \pm 0.024$ \\
\hline NGC $4736 \mathrm{E}$ & 125054.070 & +410629.06 & $<0.14$ & $0.77 \pm 0.08$ & $<0.41$ & 136.0 & 1.200 & $\cdots$ & $\cdots$ \\
\hline NGC 4736 P & 125054.541 & +410748.36 & $1.06 \pm 0.05$ & $0.30 \pm 0.07$ & $0.61 \pm 0.10$ & 136.0 & 1.069 & $-0.316 \pm 0.064$ & $0.857 \pm 0.054$ \\
\hline NGC 4736 D & 125054.565 & +410636.52 & $0.59 \pm 0.05$ & $1.05 \pm 0.08$ & $0.43 \pm 0.11$ & 136.0 & 1.029 & $0.259 \pm 0.061$ & $1.000 \pm 0.013$ \\
\hline NGC 4736 Enuc. $1 \mathrm{D}$ & 125055.99 & +410726.60 & $0.16 \pm 0.03$ & $0.33 \pm 0.05$ & $0.27 \pm 0.03$ & 90.0 & 0.869 & $0.203 \pm 0.091$ & $1.000 \pm 0.022$ \\
\hline NGC 4736 Enuc. $1 \mathrm{~A}$ & 125056.35 & +410717.00 & $1.55 \pm 0.05$ & $1.06 \pm 0.08$ & $0.88 \pm 0.04$ & 136.0 & 0.860 & $-0.238 \pm 0.022$ & $0.918 \pm 0.015$ \\
\hline NGC 4736 Enuc. $1 \mathrm{~B}$ & 125056.65 & +410704.40 & $2.79 \pm 0.05$ & $1.59 \pm 0.08$ & $1.03 \pm 0.05$ & 136.0 & 0.928 & $-0.399 \pm 0.017$ & $0.780 \pm 0.018$ \\
\hline NGC 4736 Enuc. $1 \mathrm{C}$ & 125056.83 & +410647.52 & $1.37 \pm 0.05$ & $1.13 \pm 0.08$ & $0.64 \pm 0.07$ & 136.0 & 1.133 & $-0.228 \pm 0.036$ & $0.924 \pm 0.025$ \\
\hline NGC 4736 Enuc. $1 \mathrm{E}$ & 125056.84 & +410723.00 & $0.09 \pm 0.02$ & $<0.12$ & $0.14 \pm 0.02$ & 68.0 & 1.033 & $0.205 \pm 0.126$ & $1.000 \pm 0.030$ \\
\hline NGC $4826 \mathrm{C}$ & 125643.11 & +214054.79 & $0.72 \pm 0.02$ & $0.48 \pm 0.02$ & $0.22 \pm 0.02$ & 77.0 & 0.427 & $-0.344 \pm 0.028$ & $0.833 \pm 0.026$ \\
\hline NGC 4826 B & 125643.11 & +214106.79 & $0.72 \pm 0.02$ & $0.53 \pm 0.02$ & $0.25 \pm 0.02$ & 77.0 & 0.295 & $-0.295 \pm 0.027$ & $0.874 \pm 0.021$ \\
\hline NGC 4826 A & 125643.61 & +214100.41 & $12.21 \pm 0.04$ & $4.55 \pm 0.04$ & $2.83 \pm 0.04$ & 153.0 & 0.034 & $-0.611 \pm 0.005$ & $0.494 \pm 0.008$ \\
\hline NGC $4826 \mathrm{~F}^{\mathrm{a}}$ & 125643.73 & +214100.90 & $10.97 \pm 0.12$ & $9.71 \pm 0.12$ & $3.61 \pm 0.10$ & 409.0 & 0.049 & $-0.212 \pm 0.009$ & $0.935 \pm 0.006$ \\
\hline NGC $4826 \mathrm{E}$ & 125643.84 & +214111.40 & $0.43 \pm 0.02$ & $0.46 \pm 0.02$ & $0.14 \pm 0.02$ & 77.0 & 0.558 & $-0.095 \pm 0.039$ & $1.000 \pm 0.020$ \\
\hline
\end{tabular}


Table 4

(Continued)

\begin{tabular}{|c|c|c|c|c|c|c|c|c|c|}
\hline Source ID & $\begin{array}{l}\text { R.A. } \\
\text { (J2000) }\end{array}$ & $\begin{array}{l}\text { Decl. } \\
\text { (J2000) }\end{array}$ & $\begin{array}{l}S_{3 \mathrm{GHz}} \\
(\mathrm{mJy})\end{array}$ & $\begin{array}{c}S_{15 \mathrm{GHz}} \\
(\mathrm{mJy})\end{array}$ & $\begin{array}{c}S_{33 \mathrm{GHz}} \\
(\mathrm{mJy})\end{array}$ & $\begin{array}{l}d_{\mathrm{ap}} \\
(\mathrm{pc})\end{array}$ & $\begin{array}{c}r_{\mathrm{G}} \\
(\mathrm{kpc})\end{array}$ & $\alpha$ & $f_{\mathrm{T}}^{33 \mathrm{GHz}}$ \\
\hline NGC 4826 D & 125644.34 & +214055.99 & $0.57 \pm 0.02$ & $0.33 \pm 0.02$ & $0.31 \pm 0.02$ & 77.0 & 0.250 & $-0.268 \pm 0.029$ & $\overline{0.896 \pm 0.021}$ \\
\hline NGC $5055 \mathrm{C}$ & 131546.766 & +420201.43 & $0.14 \pm 0.03$ & $<0.07$ & $<0.11$ & 154.0 & 1.349 & $\ldots$ & $\ldots$ \\
\hline NGC $5055 \mathrm{D}$ & 131547.919 & +420141.96 & $0.22 \pm 0.03$ & $0.19 \pm 0.02$ & $<0.08$ & 154.0 & 0.722 & $-0.070 \pm 0.104$ & $1.000 \pm 0.049$ \\
\hline NGC $5055 \mathrm{~A}$ & 131549.29 & +420145.82 & $1.99 \pm 0.04$ & $1.01 \pm 0.03$ & $0.33 \pm 0.04$ & 231.0 & 0.054 & $-0.475 \pm 0.022$ & $0.693 \pm 0.027$ \\
\hline NGC $5055 \mathrm{~B}$ & 131550.20 & +420101.90 & $1.20 \pm 0.03$ & $0.29 \pm 0.03$ & $<0.24$ & 154.0 & 2.805 & $-0.875 \pm 0.057$ & $0.243 \pm 0.123$ \\
\hline NGC 5055 Enuc. 1 & 131558.32 & +420027.10 & $0.47 \pm 0.04$ & $0.43 \pm 0.03$ & $0.40 \pm 0.03$ & 231.0 & 5.645 & $-0.060 \pm 0.048$ & $1.000 \pm 0.022$ \\
\hline NGC 5194 Enuc. 6 & 132939.32 & +470838.90 & $0.68 \pm 0.06$ & $0.53 \pm 0.03$ & $0.56 \pm 0.02$ & 222.0 & 12.377 & $-0.066 \pm 0.038$ & $1.000 \pm 0.018$ \\
\hline NGC 5194 Enuc. 3 B & 132943.77 & +471000.65 & $<0.19$ & $0.35 \pm 0.03$ & $0.13 \pm 0.03$ & 222.0 & 7.645 & $-1.260 \pm 0.290$ & $0.224 \pm 0.574$ \\
\hline NGC 5194 Enuc. 3 A & 132945.06 & +470958.43 & $0.81 \pm 0.08$ & $0.55 \pm 0.04$ & $0.58 \pm 0.03$ & 259.0 & 7.051 & $-0.130 \pm 0.045$ & $0.985 \pm 0.024$ \\
\hline NGC 5194 Enuc. 11 G & 132945.117 & +471332.37 & $0.29 \pm 0.04$ & $<0.11$ & $0.30 \pm 0.05$ & 148.0 & 5.358 & $0.017 \pm 0.095$ & $1.000 \pm 0.036$ \\
\hline NGC 5194 Enuc. $11 \mathrm{~A}$ & 132947.07 & +471340.72 & $0.47 \pm 0.04$ & $<0.10$ & $0.22 \pm 0.03$ & 148.0 & 4.942 & $-0.318 \pm 0.063$ & $0.855 \pm 0.054$ \\
\hline NGC 5194 Enuc. 11 B & 132947.52 & +471325.14 & $<0.13$ & $0.16 \pm 0.03$ & $0.18 \pm 0.02$ & 148.0 & 4.365 & $0.203 \pm 0.299$ & $1.000 \pm 0.073$ \\
\hline NGC 5194 Enuc. $11 \mathrm{C}$ & 132949.56 & +471328.04 & $<0.13$ & $0.10 \pm 0.03$ & $0.15 \pm 0.02$ & 148.0 & 4.057 & $0.508 \pm 0.422$ & $1.000 \pm 0.049$ \\
\hline NGC 5194 Enuc. 11 D & 132949.57 & +471400.06 & $0.85 \pm 0.04$ & $0.27 \pm 0.03$ & $0.11 \pm 0.03$ & 148.0 & 5.220 & $-0.761 \pm 0.068$ & $0.185 \pm 0.168$ \\
\hline NGC $5194 \mathrm{H}$ & 132949.96 & +471125.90 & $2.92 \pm 0.09$ & $1.22 \pm 0.06$ & $0.29 \pm 0.08$ & 296.0 & 1.968 & $-0.569 \pm 0.034$ & $0.563 \pm 0.052$ \\
\hline NGC 5194L & 132950.26 & +471146.90 & $1.66 \pm 0.07$ & $0.66 \pm 0.04$ & $0.17 \pm 0.05$ & 222.0 & 1.450 & $-0.614 \pm 0.044$ & $0.490 \pm 0.076$ \\
\hline NGC 5194 M & 132950.32 & +471137.90 & $0.68 \pm 0.03$ & $0.25 \pm 0.02$ & $0.13 \pm 0.02$ & 111.0 & 1.520 & $-0.642 \pm 0.051$ & $0.439 \pm 0.094$ \\
\hline NGC 5194 Enuc. $11 \mathrm{E}$ & 132950.48 & +471345.15 & $0.20 \pm 0.03$ & $0.23 \pm 0.02$ & $0.16 \pm 0.02$ & 111.0 & 4.641 & $-0.118 \pm 0.077$ & $0.991 \pm 0.041$ \\
\hline NGC 5194 A & 132950.69 & +471155.79 & $1.41 \pm 0.05$ & $0.55 \pm 0.03$ & $0.26 \pm 0.03$ & 148.0 & 1.185 & $-0.624 \pm 0.032$ & $0.473 \pm 0.056$ \\
\hline NGC 5194 Enuc. $11 \mathrm{~F}$ & 132950.92 & +471343.64 & $0.24 \pm 0.03$ & $0.14 \pm 0.02$ & $0.14 \pm 0.02$ & 111.0 & 4.592 & $-0.251 \pm 0.073$ & $0.908 \pm 0.053$ \\
\hline NGC $5194 \mathrm{~K}$ & 132951.43 & +471200.40 & $0.86 \pm 0.05$ & $0.66 \pm 0.03$ & $0.22 \pm 0.03$ & 148.0 & 0.876 & $-0.263 \pm 0.039$ & $0.899 \pm 0.029$ \\
\hline NGC 5194 B & 132951.57 & +471208.43 & $1.96 \pm 0.05$ & $0.64 \pm 0.03$ & $0.38 \pm 0.04$ & 148.0 & 1.045 & $-0.695 \pm 0.025$ & $0.335 \pm 0.053$ \\
\hline NGC 5194 G & 132951.79 & +471138.50 & $1.14 \pm 0.05$ & $0.48 \pm 0.03$ & $0.10 \pm 0.03$ & 148.0 & 0.629 & $-0.580 \pm 0.040$ & $0.546 \pm 0.063$ \\
\hline NGC 5194 J & 132952.23 & +471203.40 & $0.59 \pm 0.03$ & $0.14 \pm 0.02$ & $0.07 \pm 0.02$ & 111.0 & 0.777 & $-0.888 \pm 0.079$ & $0.242 \pm 0.170$ \\
\hline NGC 5194 E & 132952.59 & +471152.90 & $12.56 \pm 0.09$ & $4.37 \pm 0.05$ & $1.69 \pm 0.05$ & 296.0 & 0.379 & $-0.705 \pm 0.008$ & $0.313 \pm 0.016$ \\
\hline NGC 5194 O & 132952.61 & +471122.90 & $0.38 \pm 0.07$ & $0.47 \pm 0.04$ & $<0.13$ & 222.0 & 0.813 & $0.131 \pm 0.125$ & $1.000 \pm 0.036$ \\
\hline NGC 5194 I & 132952.76 & +471142.40 & $5.37 \pm 0.03$ & $1.42 \pm 0.02$ & $0.70 \pm 0.02$ & 111.0 & 0.037 & $-0.835 \pm 0.008$ & $0.246 \pm 0.016$ \\
\hline NGC $5194 C^{a}$ & 132952.77 & +471140.90 & $10.73 \pm 0.06$ & $2.82 \pm 0.03$ & $1.21 \pm 0.03$ & 185.0 & 0.082 & $-0.855 \pm 0.007$ & $0.245 \pm 0.014$ \\
\hline NGC 5194 Enuc. $1 \mathrm{~A}$ & 132953.22 & +471240.00 & $0.33 \pm 0.04$ & $0.10 \pm 0.02$ & $0.26 \pm 0.03$ & 148.0 & 2.368 & $-0.102 \pm 0.071$ & $0.999 \pm 0.036$ \\
\hline NGC 5194 Enuc. 4 D & 132953.90 & +471404.90 & $<0.09$ & $<0.05$ & $0.12 \pm 0.02$ & 111.0 & 5.873 & $\ldots$ & $\ldots$ \\
\hline NGC 5194N & 132954.14 & +471127.40 & $1.33 \pm 0.07$ & $0.27 \pm 0.04$ & $<0.14$ & 222.0 & 0.919 & $-0.996 \pm 0.098$ & $0.235 \pm 0.203$ \\
\hline NGC $5194 \mathrm{~F}$ & 132955.12 & +471134.39 & $0.76 \pm 0.06$ & $0.51 \pm 0.03$ & $0.31 \pm 0.04$ & 185.0 & 1.412 & $-0.305 \pm 0.053$ & $0.867 \pm 0.043$ \\
\hline NGC 5194 Enuc. 4 C & 132955.73 & +471348.40 & $0.16 \pm 0.03$ & $0.11 \pm 0.02$ & $0.11 \pm 0.02$ & 111.0 & 5.770 & $-0.149 \pm 0.099$ & $0.974 \pm 0.056$ \\
\hline NGC $5194 \mathrm{P}$ & 132955.85 & +471155.90 & $0.71 \pm 0.05$ & $0.28 \pm 0.03$ & $0.16 \pm 0.04$ & 148.0 & 2.119 & $-0.586 \pm 0.066$ & $0.536 \pm 0.107$ \\
\hline NGC 5194 Enuc. 4 E & 132956.20 & +471408.50 & $<0.09$ & $<0.05$ & $0.05 \pm 0.01$ & 111.0 & 6.690 & $\ldots$ & $\ldots$ \\
\hline NGC 5194 Enuc. 10 & 132956.73 & +471045.70 & $0.71 \pm 0.07$ & $0.35 \pm 0.04$ & $0.35 \pm 0.04$ & 222.0 & 2.829 & $-0.326 \pm 0.063$ & $0.849 \pm 0.055$ \\
\hline NGC 5194 Enuc. 4 B & 132957.62 & +471354.82 & $0.11 \pm 0.03$ & $<0.05$ & $0.07 \pm 0.02$ & 111.0 & 6.717 & $-0.217 \pm 0.156$ & $0.932 \pm 0.104$ \\
\hline NGC 5194 Enuc. 5 B & 132958.92 & +471409.40 & $0.27 \pm 0.04$ & $0.18 \pm 0.03$ & $0.15 \pm 0.03$ & 148.0 & 7.776 & $-0.264 \pm 0.100$ & $0.899 \pm 0.075$ \\
\hline NGC 5194 Enuc. $4 \mathrm{~F}$ & 132959.00 & +471409.10 & $0.21 \pm 0.03$ & $0.15 \pm 0.02$ & $0.09 \pm 0.02$ & 111.0 & 7.798 & $-0.298 \pm 0.095$ & $0.873 \pm 0.077$ \\
\hline NGC 5194 Enuc. 4 G & 132959.59 & +471359.50 & $0.56 \pm 0.04$ & $0.29 \pm 0.03$ & $0.14 \pm 0.03$ & 148.0 & 7.726 & $-0.456 \pm 0.064$ & $0.717 \pm 0.076$ \\
\hline NGC 5194 Enuc. 5 A & 132959.64 & +471359.43 & $0.64 \pm 0.05$ & $0.35 \pm 0.03$ & $0.38 \pm 0.04$ & 185.0 & 7.748 & $-0.252 \pm 0.051$ & $0.908 \pm 0.037$ \\
\hline NGC 5194 Enuc. 9 & 132959.84 & $+4711 \quad 12.60$ & $0.40 \pm 0.06$ & $0.36 \pm 0.03$ & $0.38 \pm 0.04$ & 185.0 & 4.166 & $-0.021 \pm 0.073$ & $1.000 \pm 0.030$ \\
\hline NGC 5194 Enuc. 8 C & 133000.33 & +471319.90 & $0.34 \pm 0.04$ & $0.27 \pm 0.02$ & $<0.13$ & 148.0 & 6.815 & $-0.132 \pm 0.097$ & $0.983 \pm 0.053$ \\
\hline NGC 5194 Enuc. 8 B & 133000.69 & +471306.93 & $0.67 \pm 0.07$ & $0.64 \pm 0.03$ & $0.22 \pm 0.05$ & 222.0 & 6.622 & $-0.128 \pm 0.065$ & $0.985 \pm 0.035$ \\
\hline NGC 5194 Enuc. 7 E & 133001.00 & +470928.90 & $<0.14$ & $0.19 \pm 0.02$ & $0.27 \pm 0.04$ & 148.0 & 6.204 & $0.479 \pm 0.250$ & $1.000 \pm 0.031$ \\
\hline NGC 5194 Enuc. 8 A & 133001.39 & +471251.33 & $0.55 \pm 0.07$ & $0.53 \pm 0.03$ & $0.39 \pm 0.04$ & 222.0 & 6.591 & $-0.119 \pm 0.065$ & $0.990 \pm 0.034$ \\
\hline NGC 5194 Enuc. 7 A & 133002.28 & +470947.77 & $1.20 \pm 0.07$ & $0.40 \pm 0.03$ & $0.34 \pm 0.05$ & 222.0 & 6.301 & $-0.612 \pm 0.047$ & $0.493 \pm 0.081$ \\
\hline
\end{tabular}


Table 4

(Continued)

\begin{tabular}{|c|c|c|c|c|c|c|c|c|c|}
\hline Source ID & $\begin{array}{l}\text { R.A. } \\
\text { (J2000) }\end{array}$ & $\begin{array}{l}\text { Decl. } \\
\text { (J2000) }\end{array}$ & $\begin{array}{l}S_{3 \mathrm{GHz}} \\
(\mathrm{mJy})\end{array}$ & $\begin{array}{c}S_{15 \mathrm{GHz}} \\
(\mathrm{mJy})\end{array}$ & $\begin{array}{c}S_{33 \mathrm{GHz}} \\
(\mathrm{mJy})\end{array}$ & $\begin{array}{l}d_{\mathrm{ap}} \\
(\mathrm{pc})\end{array}$ & $\begin{array}{c}r_{\mathrm{G}} \\
(\mathrm{kpc})\end{array}$ & $\alpha$ & $f_{\mathrm{T}}^{33 \mathrm{GHz}}$ \\
\hline NGC 5194 Enuc. 7 C & 133002.76 & +470956.80 & $0.41 \pm 0.05$ & $0.18 \pm 0.02$ & $0.17 \pm 0.03$ & 148.0 & 6.369 & $-0.428 \pm 0.077$ & $0.748 \pm 0.085$ \\
\hline NGC 5194 Enuc. 7 B & 133003.50 & +470941.14 & $0.32 \pm 0.06$ & $0.16 \pm 0.03$ & $<0.13$ & 185.0 & 6.961 & $-0.420 \pm 0.146$ & $0.758 \pm 0.159$ \\
\hline NGC 5194 Enuc. 7 F & 133005.06 & +471034.90 & $5.47 \pm 0.05$ & $1.81 \pm 0.03$ & $<0.32$ & 148.0 & 7.231 & $-0.688 \pm 0.010$ & $0.349 \pm 0.021$ \\
\hline NGC 5194 Enuc. 8 D & 133007.391 & +471322.05 & $0.29 \pm 0.04$ & $0.20 \pm 0.03$ & $<0.98$ & 148.0 & 10.734 & $-0.238 \pm 0.141$ & $0.918 \pm 0.099$ \\
\hline NGC 5398 & 140120.11 & -330410.66 & $2.40 \pm 0.06$ & $1.71 \pm 0.03$ & $1.68 \pm 0.03$ & 334.0 & 1.417 & $-0.152 \pm 0.013$ & $0.972 \pm 0.007$ \\
\hline NGC 5457 Enuc. 6 A & 140228.23 & +541626.55 & $1.05 \pm 0.04$ & $0.94 \pm 0.04$ & $0.55 \pm 0.04$ & 195.0 & 15.711 & $-0.179 \pm 0.028$ & $0.956 \pm 0.017$ \\
\hline NGC 5457 Enuc. $6 \mathrm{E}$ & 140228.34 & +54 1636.20 & $0.67 \pm 0.04$ & $0.45 \pm 0.03$ & $0.45 \pm 0.03$ & 162.0 & 15.525 & $-0.183 \pm 0.035$ & $0.954 \pm 0.022$ \\
\hline NGC 5457 Enuc. $6 \mathrm{~B}$ & 140229.59 & +54 16 15.85 & $2.34 \pm 0.05$ & $1.57 \pm 0.04$ & $0.76 \pm 0.05$ & 227.0 & 15.552 & $-0.323 \pm 0.019$ & $0.851 \pm 0.016$ \\
\hline NGC 5457 Enuc. 6C & 140230.59 & +541609.84 & $1.01 \pm 0.03$ & $0.74 \pm 0.03$ & $0.57 \pm 0.03$ & 130.0 & 15.416 & $-0.214 \pm 0.022$ & $0.934 \pm 0.015$ \\
\hline NGC 5457 Enuc. 6 D & 140230.64 & +541601.20 & $0.58 \pm 0.03$ & $0.37 \pm 0.03$ & $0.32 \pm 0.04$ & 130.0 & 15.563 & $-0.260 \pm 0.044$ & $0.902 \pm 0.032$ \\
\hline NGC 5457 Enuc. 6F & 140231.08 & +541549.70 & $0.21 \pm 0.02$ & $0.11 \pm 0.02$ & $<0.15$ & 97.0 & 15.678 & $-0.402 \pm 0.137$ & $0.776 \pm 0.142$ \\
\hline NGC 5457 Enuc. 2 B & 140251.05 & +542209.50 & $0.13 \pm 0.02$ & $0.12 \pm 0.01$ & $<0.11$ & 97.0 & 7.282 & $-0.086 \pm 0.127$ & $1.000 \pm 0.062$ \\
\hline NGC 5457 Enuc. $2 \mathrm{C}$ & 140259.329 & +542323.97 & $0.16 \pm 0.02$ & $0.06 \pm 0.02$ & $<0.78$ & 97.0 & 6.670 & $-0.631 \pm 0.213$ & $0.459 \pm 0.384$ \\
\hline NGC 5457 Enuc. 5 B & 140301.10 & +541430.50 & $1.48 \pm 0.03$ & $1.20 \pm 0.02$ & $1.13 \pm 0.02$ & 130.0 & 13.068 & $-0.118 \pm 0.012$ & $0.991 \pm 0.006$ \\
\hline NGC 5457 Enuc. $5 \mathrm{~A}^{\mathrm{a}}$ & 140301.17 & +541426.90 & $2.11 \pm 0.06$ & $2.16 \pm 0.04$ & $1.28 \pm 0.05$ & 260.0 & 13.180 & $-0.114 \pm 0.017$ & $0.993 \pm 0.009$ \\
\hline NGC 5457 Enuc. 5 C & 140301.24 & +541423.60 & $0.88 \pm 0.03$ & $0.62 \pm 0.02$ & $0.68 \pm 0.02$ & 130.0 & 13.282 & $-0.123 \pm 0.020$ & $0.988 \pm 0.010$ \\
\hline NGC 5457 Enuc. 1 & 140310.17 & +542058.10 & $0.10 \pm 0.02$ & $<0.04$ & $0.08 \pm 0.01$ & 97.0 & 0.756 & $-0.106 \pm 0.109$ & $0.997 \pm 0.056$ \\
\hline NGC 5457 & 140312.50 & +542056.40 & $0.87 \pm 0.05$ & $0.24 \pm 0.03$ & $0.37 \pm 0.03$ & 195.0 & 0.036 & $-0.445 \pm 0.041$ & $0.729 \pm 0.047$ \\
\hline NGC 5457 Enuc. $3 \mathrm{E}$ & 140334.039 & +541836.69 & $0.23 \pm 0.03$ & $0.19 \pm 0.05$ & $<1.61$ & 130.0 & 8.447 & $-0.121 \pm 0.197$ & $0.989 \pm 0.105$ \\
\hline NGC 5457 Enuc. 3 D & 140338.63 & +541849.30 & $0.60 \pm 0.03$ & $0.35 \pm 0.04$ & $<0.15$ & 130.0 & 9.430 & $-0.329 \pm 0.072$ & $0.846 \pm 0.063$ \\
\hline NGC 5457 Enuc. 3 A & 140339.85 & +541856.58 & $0.91 \pm 0.04$ & $0.87 \pm 0.04$ & $0.67 \pm 0.05$ & 162.0 & 9.645 & $-0.093 \pm 0.030$ & $1.000 \pm 0.015$ \\
\hline NGC 5457 Enuc. 3 B & 140341.46 & +541904.95 & $11.51 \pm 0.07$ & $9.26 \pm 0.08$ & $7.34 \pm 0.08$ & 292.0 & 9.971 & $-0.170 \pm 0.004$ & $0.962 \pm 0.003$ \\
\hline NGC 5457 Enuc. $3 \mathrm{C}$ & 140342.98 & +541924.77 & $0.97 \pm 0.04$ & $0.56 \pm 0.06$ & $0.41 \pm 0.07$ & 195.0 & 10.132 & $-0.352 \pm 0.052$ & $0.825 \pm 0.048$ \\
\hline NGC 5457 Enuc. $4 \mathrm{~A}$ & 140351.86 & +542152.80 & $0.42 \pm 0.04$ & $0.25 \pm 0.03$ & $0.23 \pm 0.03$ & 162.0 & 12.097 & $-0.271 \pm 0.061$ & $0.893 \pm 0.047$ \\
\hline NGC 5457 Enuc. 4 B & 140353.04 & +542156.31 & $0.34 \pm 0.04$ & $0.28 \pm 0.03$ & $0.26 \pm 0.03$ & 162.0 & 12.463 & $-0.109 \pm 0.062$ & $0.996 \pm 0.032$ \\
\hline NGC 5457 Enuc. $4 \mathrm{C}$ & 140353.17 & +542206.29 & $0.83 \pm 0.04$ & $0.56 \pm 0.03$ & $0.46 \pm 0.03$ & 162.0 & 12.530 & $-0.249 \pm 0.029$ & $0.910 \pm 0.021$ \\
\hline NGC 5457 Enuc. 4 D & 140354.01 & +542211.21 & $1.01 \pm 0.04$ & $0.58 \pm 0.03$ & $0.36 \pm 0.03$ & 162.0 & 12.801 & $-0.385 \pm 0.029$ & $0.794 \pm 0.029$ \\
\hline NGC 5457 Enuc. $7 \mathrm{D}^{\mathrm{a}}$ & 140429.03 & +542348.80 & $5.95 \pm 0.07$ & $4.35 \pm 0.07$ & $3.82 \pm 0.06$ & 325.0 & 23.774 & $-0.187 \pm 0.008$ & $0.951 \pm 0.005$ \\
\hline NGC 5457 Enuc. 7 B & 140429.24 & +542352.98 & $2.14 \pm 0.02$ & $0.75 \pm 0.02$ & $0.73 \pm 0.02$ & 97.0 & 23.859 & $-0.496 \pm 0.010$ & $0.667 \pm 0.013$ \\
\hline NGC 5713 A & 144010.28 & -001720.25 & $1.84 \pm 0.03$ & $0.95 \pm 0.04$ & $0.60 \pm 0.03$ & 415.0 & 2.130 & $-0.445 \pm 0.018$ & $0.730 \pm 0.020$ \\
\hline NGC $5713 \mathrm{~F}$ & 144010.34 & -001729.10 & $0.38 \pm 0.02$ & $0.32 \pm 0.03$ & $0.20 \pm 0.02$ & 311.0 & 2.197 & $-0.212 \pm 0.046$ & $0.935 \pm 0.030$ \\
\hline NGC 5713 B & 144010.74 & -001719.74 & $3.75 \pm 0.02$ & $1.70 \pm 0.03$ & $1.32 \pm 0.02$ & 311.0 & 1.329 & $-0.449 \pm 0.006$ & $0.724 \pm 0.007$ \\
\hline NGC 5713 Enuc. 2 & 144010.78 & -001750.00 & $1.22 \pm 0.05$ & $0.77 \pm 0.05$ & $0.28 \pm 0.04$ & 623.0 & 3.320 & $-0.426 \pm 0.040$ & $0.751 \pm 0.044$ \\
\hline NGC 5713 Enuc. 2 A & 144010.83 & -001736.01 & $1.93 \pm 0.04$ & $1.08 \pm 0.05$ & $0.62 \pm 0.03$ & 519.0 & 1.999 & $-0.431 \pm 0.020$ & $0.745 \pm 0.022$ \\
\hline NGC 5713 C & 144011.05 & -001719.08 & $5.39 \pm 0.02$ & $1.99 \pm 0.03$ & $1.48 \pm 0.02$ & 311.0 & 0.795 & $-0.558 \pm 0.005$ & $0.579 \pm 0.008$ \\
\hline NGC $5713 \mathrm{G}^{\mathrm{a}}$ & 144011.40 & -001720.10 & $11.25 \pm 0.12$ & $6.76 \pm 0.14$ & $2.16 \pm 0.10$ & 1556.0 & 0.174 & $-0.447 \pm 0.012$ & $0.727 \pm 0.014$ \\
\hline NGC 5713 D & 144011.41 & -001719.41 & $7.56 \pm 0.02$ & $3.24 \pm 0.03$ & $2.39 \pm 0.02$ & 311.0 & 0.163 & $-0.493 \pm 0.003$ & $0.671 \pm 0.004$ \\
\hline NGC 5713 Enuc. 1 A & 144012.10 & -001747.20 & $<0.10$ & $0.19 \pm 0.04$ & $<0.06$ & 415.0 & 3.058 & $\ldots$ & $\ldots$ \\
\hline NGC $5713 \mathrm{E}$ & 144012.74 & -001718.60 & $1.38 \pm 0.04$ & $0.86 \pm 0.05$ & $0.58 \pm 0.04$ & 519.0 & 2.158 & $-0.337 \pm 0.026$ & $0.839 \pm 0.024$ \\
\hline NGC 5866 & 150629.50 & +554547.56 & $6.88 \pm 0.05$ & $4.97 \pm 0.03$ & $2.18 \pm 0.03$ & 297.0 & 0.071 & $-0.331 \pm 0.005$ & $0.844 \pm 0.004$ \\
\hline NGC 6946 Enuc. 4 A & 203419.08 & +601009.81 & $0.38 \pm 0.03$ & $0.34 \pm 0.02$ & $0.39 \pm 0.02$ & 132.0 & 9.282 & $0.017 \pm 0.037$ & $1.000 \pm 0.014$ \\
\hline NGC 6946 Enuc. 4 B & 203419.20 & +601003.69 & $0.18 \pm 0.02$ & $0.21 \pm 0.01$ & $0.20 \pm 0.01$ & 99.0 & 9.172 & $0.038 \pm 0.056$ & $1.000 \pm 0.020$ \\
\hline NGC 6946 Enuc. $4 \mathrm{H}$ & 203421.880 & +601047.83 & $0.09 \pm 0.03$ & $0.12 \pm 0.02$ & $0.16 \pm 0.04$ & 132.0 & 9.199 & $0.216 \pm 0.172$ & $1.000 \pm 0.041$ \\
\hline NGC 6946 Enuc. 8 A & 203432.29 & +601018.61 & $2.25 \pm 0.05$ & $0.73 \pm 0.03$ & $0.82 \pm 0.05$ & 198.0 & 6.106 & $-0.550 \pm 0.021$ & $0.591 \pm 0.031$ \\
\hline NGC 6946 Enuc. 5 A & 203437.14 & +600510.18 & $<0.08$ & $<0.04$ & $0.10 \pm 0.02$ & 99.0 & 9.238 & $\cdots$ & $\cdots$ \\
\hline NGC 6946 Enuc. 8 B & 203437.489 & +600936.47 & $<0.11$ & $0.22 \pm 0.02$ & $<0.58$ & 132.0 & 6.106 & $\cdots$ & $\cdots$ \\
\hline NGC 6946 Enuc. 5 B & 203439.31 & +600452.72 & $0.33 \pm 0.04$ & $0.38 \pm 0.02$ & $0.38 \pm 0.02$ & 165.0 & 9.695 & $0.054 \pm 0.059$ & $1.000 \pm 0.020$ \\
\hline
\end{tabular}


Table 4

(Continued)

\begin{tabular}{|c|c|c|c|c|c|c|c|c|c|}
\hline Source ID & $\begin{array}{l}\text { R.A. } \\
\text { (J2000) }\end{array}$ & $\begin{array}{l}\text { Decl. } \\
\text { (J2000) }\end{array}$ & $\begin{array}{l}S_{3 \mathrm{GHz}} \\
(\mathrm{mJy})\end{array}$ & $\begin{array}{c}S_{15 \mathrm{GHz}} \\
(\mathrm{mJy})\end{array}$ & $\begin{array}{c}S_{33 \mathrm{GHz}} \\
(\mathrm{mJy})\end{array}$ & $\begin{array}{l}d_{\mathrm{ap}} \\
(\mathrm{pc})\end{array}$ & $\begin{array}{c}r_{\mathrm{G}} \\
(\mathrm{kpc})\end{array}$ & $\alpha$ & $f_{\mathrm{T}}^{33 \mathrm{GHz}}$ \\
\hline NGC 6946 Enuc. 3 A & 203449.85 & +601240.69 & $0.10 \pm 0.03$ & $0.20 \pm 0.02$ & $0.22 \pm 0.02$ & 132.0 & 7.743 & $0.259 \pm 0.118$ & $1.000 \pm 0.025$ \\
\hline NGC 6946 Enuc. 3 B & 203451.22 & +601242.36 & $0.12 \pm 0.03$ & $0.21 \pm 0.02$ & $0.20 \pm 0.02$ & 132.0 & 7.727 & $0.139 \pm 0.108$ & $1.000 \pm 0.031$ \\
\hline NGC 6946 A & 203452.18 & +600914.60 & $21.08 \pm 0.08$ & $8.50 \pm 0.05$ & $4.42 \pm 0.15$ & 297.0 & 0.041 & $-0.570 \pm 0.004$ & $0.561 \pm 0.007$ \\
\hline NGC 6946 Enuc. 3 C & 203452.32 & +601244.00 & $0.49 \pm 0.05$ & $0.47 \pm 0.03$ & $0.43 \pm 0.03$ & 198.0 & 7.736 & $-0.057 \pm 0.050$ & $1.000 \pm 0.023$ \\
\hline NGC 6946 B & 203452.90 & +600851.80 & $0.45 \pm 0.03$ & $0.39 \pm 0.02$ & $0.27 \pm 0.06$ & 99.0 & 0.857 & $-0.101 \pm 0.046$ & $1.000 \pm 0.023$ \\
\hline NGC 6946 C & 203453.59 & +600918.80 & $0.74 \pm 0.03$ & $0.62 \pm 0.02$ & $0.32 \pm 0.05$ & 99.0 & 0.356 & $-0.134 \pm 0.028$ & $0.982 \pm 0.015$ \\
\hline NGC 6946 Enuc. $6 \mathrm{~J}$ & 203503.14 & +60 1056.40 & $0.11 \pm 0.03$ & $0.07 \pm 0.01$ & $0.11 \pm 0.03$ & 99.0 & 4.357 & $-0.099 \pm 0.144$ & $1.000 \pm 0.072$ \\
\hline NGC 6946 Enuc. 6 I & 203503.75 & +601059.40 & $0.22 \pm 0.03$ & $0.10 \pm 0.01$ & $0.10 \pm 0.03$ & 99.0 & 4.524 & $-0.399 \pm 0.091$ & $0.780 \pm 0.094$ \\
\hline NGC 6946 Enuc. $6 \mathrm{H}$ & 203504.19 & +601054.60 & $0.19 \pm 0.03$ & $0.11 \pm 0.01$ & $0.15 \pm 0.03$ & 99.0 & 4.457 & $-0.154 \pm 0.087$ & $0.971 \pm 0.050$ \\
\hline NGC 6946 Enuc. $6 \mathrm{~F}$ & 203505.20 & +60 1056.40 & $0.24 \pm 0.03$ & $0.06 \pm 0.01$ & $0.12 \pm 0.02$ & 99.0 & 4.659 & $-0.406 \pm 0.089$ & $0.772 \pm 0.094$ \\
\hline NGC 6946 Enuc. 6 E & 203506.68 & $+6011 \quad 12.00$ & $<0.11$ & $0.15 \pm 0.02$ & $0.16 \pm 0.03$ & 132.0 & 5.295 & $0.085 \pm 0.298$ & $1.000 \pm 0.097$ \\
\hline NGC 6946 Enuc. 6 B & 203507.01 & +601045.30 & $0.34 \pm 0.03$ & $0.29 \pm 0.01$ & $0.19 \pm 0.03$ & 99.0 & 4.709 & $-0.151 \pm 0.052$ & $0.973 \pm 0.029$ \\
\hline NGC 6946 Enuc. 6 C & 203507.81 & +601047.10 & $0.11 \pm 0.03$ & $0.06 \pm 0.01$ & $<0.08$ & 99.0 & 4.899 & $-0.407 \pm 0.210$ & $0.772 \pm 0.221$ \\
\hline NGC 6946 Enuc. $9 \mathrm{E}$ & 203509.04 & +60 0922.95 & $0.13 \pm 0.03$ & $0.08 \pm 0.02$ & $<0.12$ & 99.0 & 4.361 & $-0.277 \pm 0.178$ & $0.889 \pm 0.137$ \\
\hline NGC 6946 Enuc. 9 D & 203510.28 & +60 0922.35 & $0.19 \pm 0.04$ & $0.22 \pm 0.02$ & $<0.14$ & 132.0 & 4.689 & $0.081 \pm 0.132$ & $1.000 \pm 0.043$ \\
\hline NGC 6946 Enuc. 9 A & 203511.24 & +600857.42 & $0.86 \pm 0.06$ & $0.74 \pm 0.03$ & $0.61 \pm 0.05$ & 198.0 & 5.111 & $-0.125 \pm 0.039$ & $0.987 \pm 0.021$ \\
\hline NGC 6946 Enuc. 6 L & 203511.31 & +60 1032.99 & $0.72 \pm 0.04$ & $0.24 \pm 0.02$ & $0.36 \pm 0.10$ & 132.0 & 5.374 & $-0.585 \pm 0.059$ & $0.538 \pm 0.095$ \\
\hline NGC 6946 Enuc. $9 \mathrm{C}$ & 203511.49 & +6009 12.45 & $0.12 \pm 0.03$ & $0.20 \pm 0.01$ & $0.11 \pm 0.03$ & 99.0 & 5.054 & $0.101 \pm 0.136$ & $1.000 \pm 0.042$ \\
\hline NGC 6946 Enuc. 9 B & 203511.69 & +600905.85 & $0.16 \pm 0.03$ & $0.17 \pm 0.01$ & $0.13 \pm 0.02$ & 99.0 & 5.154 & $-0.046 \pm 0.100$ & $1.000 \pm 0.044$ \\
\hline NGC 6946 Enuc. 7 A & 203512.96 & +600850.95 & $0.19 \pm 0.05$ & $0.75 \pm 0.02$ & $0.48 \pm 0.04$ & 165.0 & 5.630 & $-0.098 \pm 0.089$ & $1.000 \pm 0.044$ \\
\hline NGC 6946 Enuc. 7 B & 203514.11 & +60 0851.79 & $0.13 \pm 0.03$ & $0.21 \pm 0.01$ & $0.16 \pm 0.03$ & 99.0 & 5.918 & $0.089 \pm 0.114$ & $1.000 \pm 0.037$ \\
\hline NGC 6946 Enuc. 1 A & 203516.79 & +60 1100.23 & $0.69 \pm 0.03$ & $0.64 \pm 0.02$ & $0.66 \pm 0.03$ & 132.0 & 6.989 & $-0.027 \pm 0.027$ & $1.000 \pm 0.012$ \\
\hline NGC 6946 Enuc. 2 C & 203521.36 & +600951.93 & $0.17 \pm 0.03$ & $0.17 \pm 0.03$ & $<0.14$ & 132.0 & 7.548 & $-0.003 \pm 0.157$ & $1.000 \pm 0.063$ \\
\hline NGC 7331 G & 223702.38 & +34 2459.90 & $0.70 \pm 0.07$ & $<0.43$ & $<0.38$ & 562.0 & 4.675 & $\cdots$ & $\cdots$ \\
\hline NGC $7331 \mathrm{~F}$ & 223702.52 & +342517.90 & $0.69 \pm 0.05$ & $0.43 \pm 0.11$ & $<0.34$ & 422.0 & 3.998 & $-0.296 \pm 0.169$ & $0.874 \pm 0.137$ \\
\hline NGC $7331 \mathrm{H}$ & 223703.11 & +342443.40 & $1.33 \pm 0.07$ & $1.18 \pm 0.16$ & $<0.40$ & 633.0 & 3.296 & $-0.073 \pm 0.091$ & $1.000 \pm 0.043$ \\
\hline NGC 7331 I & 223703.83 & +34 2429.30 & $1.23 \pm 0.06$ & $1.47 \pm 0.13$ & $<0.38$ & 492.0 & 2.483 & $0.112 \pm 0.062$ & $1.000 \pm 0.019$ \\
\hline NGC $7331 \mathrm{~A}$ & 223704.10 & +342456.00 & $0.16 \pm 0.03$ & $0.37 \pm 0.07$ & $<0.16$ & 281.0 & 0.000 & $0.516 \pm 0.175$ & $1.000 \pm 0.020$ \\
\hline NGC $7331 \mathrm{E}$ & 223705.00 & +342508.90 & $0.91 \pm 0.07$ & $<0.48$ & $0.49 \pm 0.13$ & 633.0 & 3.053 & $-0.257 \pm 0.118$ & $0.904 \pm 0.086$ \\
\hline NGC 7331 B & 223705.36 & +342453.90 & $0.52 \pm 0.05$ & $0.80 \pm 0.11$ & $<0.26$ & 422.0 & 3.445 & $0.276 \pm 0.102$ & $1.000 \pm 0.021$ \\
\hline NGC $7331 \mathrm{D}$ & 223705.77 & +342444.90 & $0.15 \pm 0.03$ & $0.45 \pm 0.07$ & $<0.20$ & 281.0 & 4.371 & $0.673 \pm 0.170$ & $1.000 \pm 0.013$ \\
\hline NGC 7793 Enuc. $1 \mathrm{E}$ & 235748.222 & -323615.38 & $0.12 \pm 0.02$ & $0.07 \pm 0.01$ & $<0.35$ & 95.0 & 1.430 & $-0.390 \pm 0.188$ & $0.789 \pm 0.190$ \\
\hline NGC 7793 Enuc. 3 & 235748.87 & -323452.80 & $0.21 \pm 0.02$ & $0.20 \pm 0.01$ & $<0.14$ & 95.0 & 0.992 & $-0.044 \pm 0.083$ & $1.000 \pm 0.036$ \\
\hline NGC 7793 Enuc. 1 A & 235748.89 & -323658.60 & $0.09 \pm 0.03$ & $0.07 \pm 0.01$ & $<0.17$ & 114.0 & 2.587 & $-0.172 \pm 0.232$ & $0.961 \pm 0.139$ \\
\hline NGC 7793 C & 235748.94 & -323454.60 & $0.19 \pm 0.02$ & $0.17 \pm 0.01$ & $0.12 \pm 0.04$ & 95.0 & 0.941 & $-0.096 \pm 0.089$ & $1.000 \pm 0.045$ \\
\hline NGC 7793 A & 235749.41 & $-32 \quad 3526.70$ & $0.23 \pm 0.04$ & $0.20 \pm 0.02$ & $0.16 \pm 0.04$ & 152.0 & 0.097 & $-0.118 \pm 0.109$ & $0.991 \pm 0.058$ \\
\hline NGC 7793 Enuc. 1 B & 235749.68 & -323713.00 & $<0.09$ & $0.11 \pm 0.02$ & $<0.19$ & 114.0 & 2.962 & $\ldots$ & $\ldots$ \\
\hline NGC 7793 Enuc. $1 \mathrm{C}$ & 235751.10 & -323649.00 & $0.18 \pm 0.02$ & $0.14 \pm 0.01$ & $<0.21$ & 95.0 & 2.268 & $-0.153 \pm 0.105$ & $0.972 \pm 0.060$ \\
\hline NGC 7793 Enuc. 2 & 235756.15 & -323540.60 & $<0.13$ & $<0.08$ & $0.14 \pm 0.05$ & 171.0 & 1.540 & $\ldots$ & $\cdots$ \\
\hline \multicolumn{10}{|c|}{ Likely Background Galaxies } \\
\hline NGC 0925 C & 022715.30 & +333528.60 & $1.37 \pm 0.02$ & $0.68 \pm 0.02$ & $0.29 \pm 0.08$ & 133.0 & 3.375 & $-0.445 \pm 0.020$ & $0.730 \pm 0.023$ \\
\hline IC $342 \mathrm{E}$ & 034645.793 & +68 0529.95 & $0.79 \pm 0.12$ & $0.28 \pm 0.04$ & $<0.29$ & 64.0 & 0.373 & $-0.637 \pm 0.122$ & $0.448 \pm 0.223$ \\
\hline Holmberg II C & 081912.69 & +704303.80 & $0.15 \pm 0.02$ & $0.14 \pm 0.01$ & $0.17 \pm 0.02$ & 44.0 & 2.067 & $0.018 \pm 0.078$ & $1.000 \pm 0.030$ \\
\hline NGC 2841 B & 092200.988 & +505905.13 & $0.11 \pm 0.02$ & $<0.10$ & $<0.07$ & 205.0 & 2.374 & $\ldots$ & $\ldots$ \\
\hline NGC 2976 Enuc. $1 \mathrm{E}$ & 094704.737 & +675641.47 & $0.17 \pm 0.02$ & $<0.07$ & $<0.39$ & 69.0 & 2.080 & $\ldots$ & $\ldots$ \\
\hline NGC 3077 B & 100316.379 & +684342.53 & $0.06 \pm 0.02$ & $0.12 \pm 0.02$ & $<0.14$ & 56.0 & 0.456 & $0.419 \pm 0.205$ & $1.000 \pm 0.030$ \\
\hline
\end{tabular}


Table 4

(Continued)

\begin{tabular}{|c|c|c|c|c|c|c|c|c|c|}
\hline Source ID & $\begin{array}{l}\text { R.A. } \\
\text { (J2000) }\end{array}$ & $\begin{array}{l}\text { Decl. } \\
\text { (J2000) }\end{array}$ & $\begin{array}{l}S_{3 \mathrm{GHz}} \\
(\mathrm{mJy})\end{array}$ & $\begin{array}{c}S_{15 \mathrm{GHz}} \\
(\mathrm{mJy})\end{array}$ & $\begin{array}{c}S_{33 \mathrm{GHz}} \\
(\mathrm{mJy})\end{array}$ & $\begin{array}{l}d_{\mathrm{ap}} \\
(\mathrm{pc})\end{array}$ & $\begin{array}{c}r_{\mathrm{G}} \\
(\mathrm{kpc})\end{array}$ & $\alpha$ & $f_{\mathrm{T}}^{33 \mathrm{GHz}}$ \\
\hline NGC $3190 \mathrm{~B}$ & 101805.64 & +214955.91 & $2.11 \pm 0.03$ & $0.67 \pm 0.02$ & $0.50 \pm 0.03$ & 374.0 & 0.082 & $-0.664 \pm 0.015$ & $0.398 \pm 0.030$ \\
\hline NGC 3265 B & 103111.125 & +284807.09 & $0.36 \pm 0.03$ & $0.08 \pm 0.02$ & $<0.52$ & 380.0 & 5.817 & $-0.965 \pm 0.194$ & $0.237 \pm 0.406$ \\
\hline NGC $3351 \mathrm{D}$ & 104355.136 & +114205.36 & $0.32 \pm 0.04$ & $0.10 \pm 0.03$ & $<0.21$ & 181.0 & 2.472 & $-0.738 \pm 0.222$ & $0.240 \pm 0.516$ \\
\hline NGC 3627 Enuc. 1 B & 112017.291 & +125657.67 & $0.14 \pm 0.03$ & $<0.16$ & $<0.44$ & 182.0 & 7.224 & $\cdots$ & $\cdots$ \\
\hline NGC 4569 B & 123647.368 & +13 1027.07 & $0.50 \pm 0.03$ & $<0.12$ & $<0.46$ & 191.0 & 5.433 & $\cdots$ & $\cdots$ \\
\hline NGC 4625 B & 124152.424 & +411717.64 & $0.97 \pm 0.03$ & $0.27 \pm 0.02$ & $0.34 \pm 0.05$ & 180.0 & 2.434 & $-0.654 \pm 0.043$ & $0.417 \pm 0.082$ \\
\hline NGC 6946 Enuc. $4 \mathrm{~F}$ & 203423.90 & +601042.90 & $5.79 \pm 0.04$ & $1.73 \pm 0.03$ & $0.93 \pm 0.08$ & 198.0 & 8.611 & $-0.751 \pm 0.012$ & $0.208 \pm 0.029$ \\
\hline NGC 6946 Enuc. 4 G & 203426.24 & +601030.59 & $2.50 \pm 0.04$ & $0.88 \pm 0.03$ & $0.49 \pm 0.10$ & 165.0 & 7.811 & $-0.652 \pm 0.022$ & $0.420 \pm 0.042$ \\
\hline \multicolumn{10}{|c|}{ Likely Associated with Supernovae } \\
\hline NGC 2403 Enuc. 3 D & 073717.034 & +653557.79 & $<0.09$ & $1.73 \pm 0.04$ & $<2.94$ & 62.0 & 3.525 & $\cdots$ & $\cdots$ \\
\hline NGC 4736 B & 125052.026 & +410715.63 & $0.16 \pm 0.05$ & $<0.19$ & $<0.15$ & 136.0 & 0.280 & $\cdots$ & $\cdots$ \\
\hline NGC $4736 \mathrm{C}$ & 125052.522 & +410701.94 & $0.16 \pm 0.05$ & $<0.20$ & $<0.15$ & 136.0 & 0.350 & $\cdots$ & $\cdots$ \\
\hline NGC 5194 D & 132955.79 & +471145.19 & $1.22 \pm 0.06$ & $0.63 \pm 0.03$ & $0.82 \pm 0.05$ & 185.0 & 1.909 & $-0.221 \pm 0.030$ & $0.929 \pm 0.020$ \\
\hline NGC 5194 Enuc. 7 D & 133005.12 & +471010.90 & $1.54 \pm 0.05$ & $0.17 \pm 0.02$ & $<0.15$ & 148.0 & 7.398 & $-1.377 \pm 0.084$ & $0.221 \pm 0.165$ \\
\hline NGC $6946 \mathrm{~F}$ & 203450.927 & +601020.35 & $0.20 \pm 0.04$ & $0.11 \pm 0.03$ & $<2.45$ & 132.0 & 2.527 & $-0.386 \pm 0.223$ & $0.792 \pm 0.223$ \\
\hline NGC 6946 Enuc. 6 D & 203508.09 & +6011 13.50 & $0.28 \pm 0.04$ & $0.35 \pm 0.02$ & $0.22 \pm 0.04$ & 132.0 & 5.557 & $0.032 \pm 0.078$ & $1.000 \pm 0.029$ \\
\hline NGC 6946 Enuc. 2 B & 203525.29 & +600958.64 & $1.23 \pm 0.06$ & $1.42 \pm 0.04$ & $1.89 \pm 0.05$ & 231.0 & 8.572 & $0.187 \pm 0.023$ & $1.000 \pm 0.006$ \\
\hline NGC $7331 \mathrm{C}$ & 223705.60 & +342432.90 & $0.79 \pm 0.07$ & $1.85 \pm 0.15$ & $0.72 \pm 0.15$ & 562.0 & 3.813 & $0.349 \pm 0.065$ & $1.000 \pm 0.011$ \\
\hline NGC 7793 B & 235747.35 & -323524.60 & $0.14 \pm 0.02$ & $<0.04$ & $<0.11$ & 95.0 & 0.591 & $\ldots$ & $\ldots$ \\
\hline \multicolumn{10}{|c|}{ Likely AME Candidates } \\
\hline NGC 2403 Enuc. 5 D & 073620.57 & +653708.50 & $0.64 \pm 0.03$ & $0.63 \pm 0.02$ & $0.62 \pm 0.02$ & 62.0 & 3.362 & $-0.014 \pm 0.022$ & $1.000 \pm 0.009$ \\
\hline NGC 2403 Enuc. 2 E & 073657.88 & +653724.39 & $1.17 \pm 0.03$ & $0.96 \pm 0.03$ & $1.19 \pm 0.08$ & 62.0 & 2.684 & $-0.075 \pm 0.019$ & $1.000 \pm 0.009$ \\
\hline NGC 2403 Enuc. 3 A & 073705.20 & +653641.74 & $0.39 \pm 0.03$ & $0.40 \pm 0.02$ & $0.52 \pm 0.03$ & 62.0 & 2.637 & $0.108 \pm 0.039$ & $1.000 \pm 0.012$ \\
\hline NGC 2403 Enuc. 3 C & 073707.86 & +653638.56 & $2.51 \pm 0.03$ & $1.91 \pm 0.02$ & $1.89 \pm 0.03$ & 62.0 & 2.921 & $-0.135 \pm 0.007$ & $0.982 \pm 0.004$ \\
\hline NGC 3627 Enuc. 1 D & 112013.857 & +125709.26 & $0.41 \pm 0.03$ & $0.56 \pm 0.05$ & $0.61 \pm 0.13$ & 182.0 & 7.171 & $0.185 \pm 0.067$ & $1.000 \pm 0.017$ \\
\hline NGC 3627 Enuc. $1 \mathrm{C}$ & 112014.093 & +125659.60 & $0.45 \pm 0.05$ & $0.63 \pm 0.08$ & $0.96 \pm 0.30$ & 273.0 & 7.467 & $0.248 \pm 0.094$ & $1.000 \pm 0.021$ \\
\hline NGC 4254 Enuc. 2 A & 121846.14 & +142419.18 & $0.12 \pm 0.03$ & $0.15 \pm 0.03$ & $0.21 \pm 0.02$ & 279.0 & 4.669 & $0.252 \pm 0.109$ & $1.000 \pm 0.024$ \\
\hline NGC 4254 Enuc. 1 A & 121849.21 & +142357.78 & $0.34 \pm 0.03$ & $0.14 \pm 0.02$ & $0.23 \pm 0.03$ & 279.0 & 4.437 & $-0.225 \pm 0.059$ & $0.926 \pm 0.040$ \\
\hline NGC $4631 \mathrm{C}$ & 124205.62 & +323229.46 & $1.75 \pm 0.03$ & $0.87 \pm 0.03$ & $1.25 \pm 0.03$ & 148.0 & 1.354 & $-0.174 \pm 0.012$ & $0.959 \pm 0.007$ \\
\hline NGC $4631 \mathrm{D}$ & 124206.24 & +323231.70 & $1.54 \pm 0.03$ & $0.53 \pm 0.03$ & $0.94 \pm 0.03$ & 148.0 & 1.525 & $-0.248 \pm 0.015$ & $0.911 \pm 0.011$ \\
\hline NGC $4631 \mathrm{E}$ & 124207.18 & +323233.90 & $0.94 \pm 0.02$ & $0.46 \pm 0.03$ & $0.79 \pm 0.03$ & 111.0 & 1.740 & $-0.109 \pm 0.016$ & $0.995 \pm 0.008$ \\
\hline NGC $4631 \mathrm{~F}$ & 124207.89 & +323233.42 & $6.83 \pm 0.04$ & $3.27 \pm 0.05$ & $3.98 \pm 0.06$ & 222.0 & 1.366 & $-0.285 \pm 0.006$ & $0.882 \pm 0.005$ \\
\hline NGC 4631 Enuc. 2 A & 124221.41 & +323306.38 & $0.60 \pm 0.04$ & $0.23 \pm 0.02$ & $0.43 \pm 0.02$ & 185.0 & 9.993 & $-0.171 \pm 0.034$ & $0.961 \pm 0.020$ \\
\hline NGC 4725 B & 125028.49 & +253022.26 & $0.15 \pm 0.02$ & $0.18 \pm 0.01$ & $0.35 \pm 0.02$ & 173.0 & 1.930 & $0.414 \pm 0.052$ & $1.000 \pm 0.008$ \\
\hline NGC 5194 Enuc. 2 & 132944.09 & +471022.86 & $1.33 \pm 0.08$ & $0.46 \pm 0.03$ & $0.58 \pm 0.04$ & 259.0 & 6.852 & $-0.399 \pm 0.035$ & $0.780 \pm 0.037$ \\
\hline NGC 5194 Enuc. $1 \mathrm{C}$ & 132949.39 & +471240.90 & $0.54 \pm 0.05$ & $0.46 \pm 0.03$ & $0.72 \pm 0.05$ & 148.0 & 2.587 & $0.089 \pm 0.045$ & $1.000 \pm 0.015$ \\
\hline NGC 5194 Enuc. 1 B & 132951.98 & +471243.90 & $0.50 \pm 0.05$ & $0.48 \pm 0.02$ & $0.55 \pm 0.03$ & 148.0 & 2.327 & $0.048 \pm 0.043$ & $1.000 \pm 0.015$ \\
\hline NGC 5194 Enuc. 4 A & 132955.50 & +471401.50 & $0.71 \pm 0.04$ & $0.29 \pm 0.02$ & $0.40 \pm 0.02$ & 148.0 & 6.192 & $-0.253 \pm 0.031$ & $0.907 \pm 0.023$ \\
\hline NGC 5457 Enuc. 2 A & 140255.08 & +542227.44 & $0.40 \pm 0.04$ & $0.27 \pm 0.02$ & $0.37 \pm 0.03$ & 162.0 & 6.429 & $-0.060 \pm 0.052$ & $1.000 \pm 0.024$ \\
\hline NGC 5457 Enuc. 7 A & 140428.61 & +542352.40 & $1.07 \pm 0.02$ & $0.55 \pm 0.02$ & $0.71 \pm 0.02$ & 97.0 & 23.668 & $-0.197 \pm 0.013$ & $0.945 \pm 0.008$ \\
\hline NGC 5457 Enuc. $7 \mathrm{C}$ & 140429.45 & +542347.11 & $3.56 \pm 0.03$ & $1.80 \pm 0.03$ & $1.93 \pm 0.02$ & 130.0 & 23.894 & $-0.285 \pm 0.006$ & $0.883 \pm 0.005$ \\
\hline NGC 6946 Enuc. 4 C & 203419.88 & +601006.42 & $1.58 \pm 0.03$ & $1.42 \pm 0.02$ & $1.56 \pm 0.02$ & 132.0 & 9.032 & $0.001 \pm 0.009$ & $1.000 \pm 0.004$ \\
\hline NGC 6946 Enuc. 4 D & 203421.39 & +6010 17.87 & $0.11 \pm 0.03$ & $0.81 \pm 0.02$ & $0.69 \pm 0.02$ & 132.0 & 8.808 & $-0.076 \pm 0.043$ & $1.000 \pm 0.021$ \\
\hline NGC 6946 Enuc. 4 E & 203422.72 & +60 1034.04 & $1.35 \pm 0.03$ & $1.34 \pm 0.02$ & $1.29 \pm 0.03$ & 132.0 & 8.741 & $-0.015 \pm 0.013$ & $1.000 \pm 0.005$ \\
\hline
\end{tabular}


Table 4

(Continued)

\begin{tabular}{|c|c|c|c|c|c|c|c|c|c|}
\hline Source ID & $\begin{array}{l}\text { R.A. } \\
\text { (J2000) }\end{array}$ & $\begin{array}{l}\text { Decl. } \\
(\mathrm{J} 2000)\end{array}$ & $\begin{array}{l}S_{3 \mathrm{GHz}} \\
(\mathrm{mJy})\end{array}$ & $\begin{array}{c}S_{15 \mathrm{GHz}} \\
(\mathrm{mJy})\end{array}$ & $\begin{array}{c}S_{33 \mathrm{GHz}} \\
(\mathrm{mJy})\end{array}$ & $\begin{array}{l}d_{\mathrm{ap}} \\
(\mathrm{pc})\end{array}$ & $\begin{array}{c}r_{\mathrm{G}} \\
(\mathrm{kpc})\end{array}$ & $\alpha$ & $f_{\mathrm{T}}^{33 \mathrm{GHz}}$ \\
\hline NGC 6946 E & 203451.30 & +600938.30 & $0.73 \pm 0.05$ & $0.71 \pm 0.03$ & $1.27 \pm 0.11$ & 165.0 & 0.972 & $0.121 \pm 0.040$ & $1.000 \pm 0.012$ \\
\hline NGC 6946 D & 203452.78 & +600930.50 & $0.40 \pm 0.04$ & $0.51 \pm 0.02$ & $0.54 \pm 0.07$ & 132.0 & 0.601 & $0.132 \pm 0.058$ & $1.000 \pm 0.017$ \\
\hline NGC 6946 Enuc. $6 \mathrm{M}$ & 203500.77 & +601130.29 & $0.50 \pm 0.04$ & $0.09 \pm 0.02$ & $0.45 \pm 0.10$ & 132.0 & 5.163 & $-0.301 \pm 0.088$ & $0.870 \pm 0.072$ \\
\hline NGC 6946 Enuc. $6 \mathrm{G}$ & 203506.00 & +601100.60 & $0.79 \pm 0.03$ & $0.49 \pm 0.01$ & $0.54 \pm 0.02$ & 99.0 & 4.894 & $-0.204 \pm 0.021$ & $0.941 \pm 0.014$ \\
\hline NGC 6946 Enuc. $6 \mathrm{~K}$ & 203506.20 & +601055.50 & $0.70 \pm 0.03$ & $0.38 \pm 0.01$ & $0.45 \pm 0.02$ & 99.0 & 4.801 & $-0.246 \pm 0.025$ & $0.912 \pm 0.018$ \\
\hline NGC 6946 Enuc. $6 \mathrm{~A}^{\mathrm{a}}$ & 203506.24 & +601058.32 & $1.84 \pm 0.05$ & $1.08 \pm 0.03$ & $1.20 \pm 0.05$ & 198.0 & 4.876 & $-0.232 \pm 0.019$ & $0.922 \pm 0.013$ \\
\hline NGC 6946 Enuc. 1 B & 203511.25 & +601033.89 & $0.72 \pm 0.03$ & $0.36 \pm 0.02$ & $0.66 \pm 0.08$ & 132.0 & 5.374 & $-0.287 \pm 0.039$ & $0.881 \pm 0.031$ \\
\hline NGC 6946 Enuc. 2 A & 203523.61 & +600948.93 & $0.11 \pm 0.03$ & $0.31 \pm 0.02$ & $0.36 \pm 0.04$ & 132.0 & 8.130 & $0.379 \pm 0.112$ & $1.000 \pm 0.018$ \\
\hline NGC 7793 Enuc. 1 D & 235746.742 & -323607.00 & $0.23 \pm 0.02$ & $0.10 \pm 0.02$ & $<0.81$ & 95.0 & 1.393 & $-0.486 \pm 0.122$ & $0.680 \pm 0.155$ \\
\hline
\end{tabular}

Notes.

${ }^{\mathrm{a}}$ The aperture used for this region contained multiple smaller individual regions. 
Table 5

Region Photometry and Derived Parameters at 7" Angular Resolution

\begin{tabular}{|c|c|c|c|c|c|c|c|c|c|}
\hline Source ID & $\begin{array}{c}\text { R.A. } \\
\text { (J2000) }\end{array}$ & $\begin{array}{c}\text { Decl. } \\
\text { (J2000) }\end{array}$ & $\begin{array}{l}S_{3 \mathrm{GHz}} \\
(\mathrm{mJy})\end{array}$ & $\begin{array}{c}S_{15 \mathrm{GHz}} \\
(\mathrm{mJy})\end{array}$ & $\begin{array}{c}S_{33 \mathrm{GHz}} \\
(\mathrm{mJy})\end{array}$ & $\begin{array}{l}d_{\mathrm{ap}} \\
(\mathrm{pc})\end{array}$ & $\begin{array}{c}r_{\mathrm{G}} \\
(\mathrm{kpc})\end{array}$ & $\alpha$ & $f_{\mathrm{T}}^{33 \mathrm{GHz}}$ \\
\hline \multicolumn{10}{|c|}{ Star-forming Regions } \\
\hline NGC 0337 a & 005950.018 & -073433.9 & $0.53 \pm 0.02$ & $0.37 \pm 0.01$ & $0.23 \pm 0.01$ & 655.0 & 0.945 & $-0.30 \pm 0.03$ & $0.87 \pm 0.02$ \\
\hline NGC 0337 b & 005950.683 & -073457.6 & $1.70 \pm 0.05$ & $0.91 \pm 0.03$ & $0.61 \pm 0.02$ & 655.0 & 1.966 & $-0.42 \pm 0.02$ & $0.76 \pm 0.02$ \\
\hline NGC 0337 c & 005951.995 & -073454.9 & $0.13 \pm 0.01$ & $0.12 \pm 0.01$ & $0.10 \pm 0.02$ & 655.0 & 3.227 & $-0.10 \pm 0.07$ & $1.00 \pm 0.03$ \\
\hline NGC $0337 \mathrm{~d}$ & 005952.156 & -073438.2 & $0.25 \pm 0.02$ & $0.25 \pm 0.01$ & $0.21 \pm 0.02$ & 655.0 & 4.061 & $-0.05 \pm 0.04$ & $1.00 \pm 0.02$ \\
\hline NGC 0628 Enuc. 4 & 013635.718 & +155007.25 & $0.14 \pm 0.01$ & $0.13 \pm 0.01$ & $0.12 \pm 0.01$ & 244.0 & 7.608 & $-0.04 \pm 0.04$ & $1.00 \pm 0.02$ \\
\hline NGC 0628 Enuc. 2 & 013637.645 & +154507.2 & $0.26 \pm 0.01$ & $0.15 \pm 0.01$ & $0.18 \pm 0.01$ & 244.0 & 4.468 & $-0.22 \pm 0.03$ & $0.93 \pm 0.02$ \\
\hline NGC 0628 Enuc. 3 & 013638.779 & +154423.2 & $0.27 \pm 0.01$ & $0.21 \pm 0.01$ & $0.19 \pm 0.02$ & 244.0 & 5.720 & $-0.16 \pm 0.03$ & $0.97 \pm 0.02$ \\
\hline NGC 0628 & 013641.7 & +154659 & $<0.03$ & $<0.02$ & $<0.04$ & 244.0 & 0.071 & $\cdots$ & $\cdots$ \\
\hline NGC 0628 Enuc. 1 & 013645.266 & +154748.3 & $0.55 \pm 0.02$ & $0.42 \pm 0.01$ & $0.29 \pm 0.02$ & 244.0 & 2.478 & $-0.22 \pm 0.02$ & $0.93 \pm 0.02$ \\
\hline NGC 0855 & 021403.677 & +27 5237.85 & $0.47 \pm 0.02$ & $0.28 \pm 0.01$ & $0.22 \pm 0.01$ & 330.0 & 0.202 & $-0.31 \pm 0.02$ & $0.86 \pm 0.02$ \\
\hline NGC 0925 & 022717 & +333443 & $0.06 \pm 0.01$ & $0.04 \pm 0.01$ & $<0.03$ & 310.0 & 0.183 & $-0.30 \pm 0.14$ & $0.87 \pm 0.12$ \\
\hline NGC 1097 Enuc. 2 & 024614.4 & -301503.992 & $<0.19$ & $<0.04$ & $<0.06$ & 482.0 & 7.435 & $\ldots$ & $\ldots$ \\
\hline NGC 1097 & 024618.984 & -301628.8 & $5.57 \pm 0.18$ & $2.55 \pm 0.08$ & $2.29 \pm 0.09$ & 482.0 & 0.020 & $-0.40 \pm 0.02$ & $0.78 \pm 0.02$ \\
\hline NGC 1097 Enuc. 1 a & 024622.557 & -301729.9 & $<0.21$ & $0.08 \pm 0.01$ & $0.08 \pm 0.02$ & 482.0 & 5.416 & $-0.07 \pm 0.41$ & $1.00 \pm 0.20$ \\
\hline NGC 1097 Enuc. $1 \mathrm{~b}$ & 024622.927 & -301748.1 & $0.33 \pm 0.07$ & $<0.04$ & $0.11 \pm 0.02$ & 482.0 & 6.817 & $-0.48 \pm 0.11$ & $0.69 \pm 0.14$ \\
\hline NGC 1097 Enuc. $1 \mathrm{c}$ & 024624.062 & -301750.9 & $<0.21$ & $<0.04$ & $0.07 \pm 0.02$ & 482.0 & 7.392 &.. & $\ldots$ \\
\hline NGC 1266 & 031600.76 & -022537.1 & $23.46 \pm 0.70$ & $6.94 \pm 0.21$ & $4.13 \pm 0.13$ & 1038.0 & 0.270 & $-0.73 \pm 0.02$ & $0.26 \pm 0.04$ \\
\hline NGC 1377 & 033638.9 & -205406 & $<0.06$ & $<0.03$ & $<0.06$ & 835.0 & 0.580 & $\ldots$ & $\ldots$ \\
\hline NGC 1482 & 035438.966 & -203007.8 & $33.61 \pm 1.01$ & $9.61 \pm 0.29$ & $6.78 \pm 0.21$ & 767.0 & 0.124 & $-0.69 \pm 0.02$ & $0.35 \pm 0.04$ \\
\hline NGC 2403 Enuc. 6 & 073628.693 & +653349.4 & $0.34 \pm 0.01$ & $0.32 \pm 0.01$ & $0.30 \pm 0.01$ & 109.0 & 5.380 & $-0.06 \pm 0.02$ & $1.00 \pm 0.01$ \\
\hline NGC 2403 Enuc. 1 a & 073642.061 & +653651.898 & $0.26 \pm 0.01$ & $0.20 \pm 0.01$ & $0.13 \pm 0.01$ & 109.0 & 1.087 & $-0.22 \pm 0.04$ & $0.93 \pm 0.02$ \\
\hline NGC 2403 Enuc. $1 \mathrm{~b}$ & 073645.5 & +653700.9 & $0.66 \pm 0.02$ & $0.59 \pm 0.02$ & $0.55 \pm 0.02$ & 109.0 & 1.192 & $-0.07 \pm 0.02$ & $1.00 \pm 0.01$ \\
\hline NGC 2403 Enuc. 2 a & 073649.115 & +653651.697 & $0.35 \pm 0.01$ & $0.30 \pm 0.01$ & $0.25 \pm 0.01$ & 109.0 & 1.113 & $-0.13 \pm 0.03$ & $0.98 \pm 0.01$ \\
\hline NGC 2403 & 073650 & +653604 & $<0.03$ & $<0.02$ & $<0.02$ & 109.0 & 0.000 & $\ldots$ & $\cdots$ \\
\hline NGC 2403 Enuc. 2 b & 073652.361 & +653646.9 & $0.64 \pm 0.02$ & $0.50 \pm 0.02$ & $0.49 \pm 0.02$ & 109.0 & 1.249 & $-0.12 \pm 0.02$ & $0.99 \pm 0.01$ \\
\hline NGC 2403 Enuc. 4 & 073718.19 & +653348.1 & $0.34 \pm 0.01$ & $0.33 \pm 0.01$ & $0.33 \pm 0.01$ & 109.0 & 3.455 & $-0.01 \pm 0.02$ & $1.00 \pm 0.01$ \\
\hline Holmberg II & 081913.058 & +704308 & $0.20 \pm 0.01$ & $0.19 \pm 0.01$ & $0.21 \pm 0.01$ & 104.0 & 0.738 & $0.01 \pm 0.04$ & $1.00 \pm 0.01$ \\
\hline NGC 2798 & 091722.854 & +420000.4 & $15.27 \pm 0.46$ & $5.71 \pm 0.17$ & $2.06 \pm 0.06$ & 876.0 & 0.144 & $-0.80 \pm 0.02$ & $0.08 \pm 0.05$ \\
\hline NGC 2841 & 092202.668 & +505835.7 & $0.92 \pm 0.03$ & $0.89 \pm 0.03$ & $0.56 \pm 0.02$ & 479.0 & 0.155 & $-0.18 \pm 0.02$ & $0.95 \pm 0.01$ \\
\hline NGC 2976 Enuc. 1 a & 094705.192 & +675551.999 & $0.54 \pm 0.02$ & $0.36 \pm 0.01$ & $0.35 \pm 0.02$ & 120.0 & 1.420 & $-0.21 \pm 0.02$ & $0.94 \pm 0.01$ \\
\hline NGC 2976 Enuc. $1 \mathrm{~b}$ & 094707.64 & +675554.7 & $1.23 \pm 0.04$ & $1.01 \pm 0.03$ & $0.86 \pm 0.03$ & 120.0 & 1.201 & $-0.14 \pm 0.02$ & $0.98 \pm 0.01$ \\
\hline NGC 2976 & 094713.491 & +675453.999 & $0.11 \pm 0.01$ & $0.08 \pm 0.01$ & $0.09 \pm 0.01$ & 120.0 & 0.462 & $-0.12 \pm 0.07$ & $0.99 \pm 0.04$ \\
\hline NGC 2976 Enuc. 2 a & 094723.834 & +675354.9 & $0.59 \pm 0.02$ & $0.51 \pm 0.02$ & $0.45 \pm 0.02$ & 120.0 & 1.394 & $-0.11 \pm 0.02$ & $0.99 \pm 0.01$ \\
\hline NGC 2976 Enuc. 2 b & 094723.941 & +675402.1 & $0.28 \pm 0.01$ & $0.23 \pm 0.01$ & $0.21 \pm 0.02$ & 120.0 & 1.310 & $-0.13 \pm 0.03$ & $0.98 \pm 0.02$ \\
\hline NGC 3049 & 095449.559 & +09 1616.1 & $1.35 \pm 0.04$ & $0.75 \pm 0.02$ & $0.55 \pm 0.02$ & 652.0 & 0.103 & $-0.38 \pm 0.02$ & $0.80 \pm 0.02$ \\
\hline NGC 3077 & 100319.15 & +684359.90 & $3.62 \pm 0.11$ & $2.31 \pm 0.07$ & $1.89 \pm 0.06$ & 130.0 & 0.045 & $-0.27 \pm 0.02$ & $0.89 \pm 0.01$ \\
\hline NGC 3190 & 101805.643 & +214955.9 & $1.08 \pm 0.03$ & $0.50 \pm 0.02$ & $0.27 \pm 0.01$ & 655.0 & 0.098 & $-0.55 \pm 0.02$ & $0.59 \pm 0.03$ \\
\hline NGC 3184 & $1018 \quad 16.94$ & +412527 & $0.35 \pm 0.01$ & $0.18 \pm 0.01$ & $0.14 \pm 0.01$ & 397.0 & 0.063 & $-0.40 \pm 0.03$ & $0.78 \pm 0.03$ \\
\hline NGC 3198 & 101954.986 & +453259.3 & $0.63 \pm 0.02$ & $0.31 \pm 0.01$ & $0.12 \pm 0.01$ & 479.0 & 0.116 & $-0.51 \pm 0.03$ & $0.65 \pm 0.04$ \\
\hline IC $2574 \mathrm{a}$ & 102843.712 & +682826.296 & $0.29 \pm 0.01$ & $0.20 \pm 0.01$ & $0.28 \pm 0.02$ & 129.0 & 6.253 & $-0.11 \pm 0.03$ & $0.99 \pm 0.02$ \\
\hline IC $2574 \mathrm{~b}$ & 102848.4 & +68 2803.5 & $0.29 \pm 0.01$ & $0.23 \pm 0.01$ & $0.24 \pm 0.01$ & 129.0 & 5.254 & $-0.10 \pm 0.03$ & $1.00 \pm 0.01$ \\
\hline NGC 3265 & 103106.768 & +284748 & $1.97 \pm 0.06$ & $0.77 \pm 0.02$ & $0.44 \pm 0.02$ & 665.0 & 0.087 & $-0.62 \pm 0.02$ & $0.48 \pm 0.03$ \\
\hline NGC 3351 a & 104357.677 & +114208 & $2.84 \pm 0.09$ & $1.14 \pm 0.04$ & $0.78 \pm 0.03$ & 317.0 & 0.230 & $-0.54 \pm 0.02$ & $0.60 \pm 0.03$ \\
\hline NGC $3351 \mathrm{~b}$ & 104357.8 & +114218.5 & $3.28 \pm 0.10$ & $1.37 \pm 0.04$ & $0.91 \pm 0.03$ & 317.0 & 0.258 & $-0.54 \pm 0.02$ & $0.61 \pm 0.03$ \\
\hline NGC 3521 Enuc. 1 & 110546.3 & -000408.992 & $0.05 \pm 0.02$ & $0.13 \pm 0.02$ & $<0.07$ & 380.0 & 9.929 & $0.56 \pm 0.21$ & $1.00 \pm 0.02$ \\
\hline NGC 3521 Enuc. 3 & 110547.6 & +000033.004 & $<0.05$ & $0.08 \pm 0.01$ & $<0.05$ & 380.0 & 9.509 & $\cdots$ & $\cdots$ \\
\hline NGC 3521 & 110548.9 & -000206 & $<0.06$ & $<0.10$ & $<0.05$ & 380.0 & 0.625 & $\ldots$ & $\cdots$ \\
\hline NGC 3521 Enuc. 2 a & 110549.34 & -000324.2 & $0.29 \pm 0.02$ & $0.13 \pm 0.02$ & $0.13 \pm 0.03$ & 380.0 & 4.302 & $-0.39 \pm 0.07$ & $0.79 \pm 0.07$ \\
\hline NGC 3521 Enuc. 2 b & 110549.94 & -000355.9 & $<0.06$ & $<0.06$ & $0.11 \pm 0.03$ & 380.0 & 6.044 & $\ldots$ & $\ldots$ \\
\hline NGC 3621 & 111816 & -324842 & $0.06 \pm 0.02$ & $<0.04$ & $<0.07$ & 222.0 & 0.348 & $\ldots$ & $\ldots$ \\
\hline NGC 3627 & 112015 & +125929.4 & $4.75 \pm 0.14$ & $1.73 \pm 0.05$ & $1.09 \pm 0.04$ & 318.0 & 0.028 & $-0.62 \pm 0.02$ & $0.48 \pm 0.03$ \\
\hline NGC 3627 Enuc. 1 & 112016.323 & +125749.2 & $1.33 \pm 0.04$ & $1.03 \pm 0.03$ & $0.86 \pm 0.03$ & 318.0 & 4.712 & $-0.18 \pm 0.02$ & $0.96 \pm 0.01$ \\
\hline NGC 3627 Enuc. 2 & 112016.464 & +125843.4 & $4.56 \pm 0.14$ & $2.52 \pm 0.08$ & $1.97 \pm 0.06$ & 318.0 & 2.746 & $-0.35 \pm 0.02$ & $0.82 \pm 0.02$ \\
\hline NGC 3773 & 113813.02 & +120643.8 & $0.85 \pm 0.03$ & $0.45 \pm 0.02$ & $0.39 \pm 0.02$ & 421.0 & 0.023 & $-0.34 \pm 0.02$ & $0.84 \pm 0.02$ \\
\hline NGC 3938 b & 115248.191 & +440705.9 & $<0.03$ & $<0.02$ & $0.08 \pm 0.01$ & 607.0 & 1.407 & $\cdots$ & $\cdots$ \\
\hline NGC 3938 a & 115249.5 & +440714 & $<0.03$ & $<0.02$ & $<0.03$ & 607.0 & 0.140 & $\cdots$ & $\cdots$ \\
\hline NGC 3938 Enuc. 2 a & 115300.056 & +440800 & $0.09 \pm 0.01$ & $0.06 \pm 0.01$ & $0.10 \pm 0.01$ & 607.0 & 11.158 & $0.00 \pm 0.07$ & $1.00 \pm 0.03$ \\
\hline NGC 3938 Enuc. 2 b & 115300.195 & +440748.3 & $0.15 \pm 0.01$ & $0.13 \pm 0.01$ & $0.11 \pm 0.01$ & 607.0 & 11.049 & $-0.11 \pm 0.05$ & $0.99 \pm 0.03$ \\
\hline NGC 4254 Enuc. 2 a & 121845.777 & +142410.4 & $0.04 \pm 0.01$ & $0.05 \pm 0.01$ & $0.09 \pm 0.01$ & 489.0 & 5.342 & $0.46 \pm 0.12$ & $1.00 \pm 0.02$ \\
\hline NGC 4254 Enuc. 2 b & 121846.128 & +142418.8 & $0.06 \pm 0.01$ & $0.07 \pm 0.01$ & $0.12 \pm 0.01$ & 489.0 & 4.698 & $0.32 \pm 0.08$ & $1.00 \pm 0.01$ \\
\hline NGC 4254 a & 121848.677 & +142442.5 & $0.17 \pm 0.01$ & $0.04 \pm 0.01$ & $0.07 \pm 0.01$ & 489.0 & 1.553 & $-0.52 \pm 0.07$ & $0.64 \pm 0.09$ \\
\hline NGC 4254 b & 121849.668 & +142459 & $0.14 \pm 0.01$ & $0.11 \pm 0.01$ & $0.11 \pm 0.01$ & 489.0 & 0.111 & $-0.09 \pm 0.05$ & $1.00 \pm 0.02$ \\
\hline
\end{tabular}


Table 5

(Continued)

\begin{tabular}{|c|c|c|c|c|c|c|c|c|c|}
\hline Source ID & $\begin{array}{l}\text { R.A. } \\
\text { (J2000) }\end{array}$ & $\begin{array}{c}\text { Decl. } \\
(\mathrm{J} 2000)\end{array}$ & $\begin{array}{l}S_{3 \mathrm{GHz}} \\
(\mathrm{mJy})\end{array}$ & $\begin{array}{c}S_{15 \mathrm{GHz}} \\
(\mathrm{mJy})\end{array}$ & $\begin{array}{c}S_{33 \mathrm{GHz}} \\
(\mathrm{mJy})\end{array}$ & $\begin{array}{c}d_{\mathrm{ap}} \\
(\mathrm{pc})\end{array}$ & $\begin{array}{c}r_{\mathrm{G}} \\
(\mathrm{kpc})\end{array}$ & $\alpha$ & $f_{\mathrm{T}}^{33 \mathrm{GHz}}$ \\
\hline NGC 4254 Enuc. $1 \mathrm{~b}$ & 121850.009 & +142406.9 & $0.07 \pm 0.01$ & $0.05 \pm 0.01$ & $0.15 \pm 0.01$ & 489.0 & 3.886 & $0.38 \pm 0.07$ & $1.00 \pm 0.01$ \\
\hline NGC $4254 \mathrm{c}$ & 121850.102 & +142511.6 & $0.04 \pm 0.01$ & $0.09 \pm 0.01$ & $0.07 \pm 0.01$ & 489.0 & 0.960 & $0.14 \pm 0.13$ & $1.00 \pm 0.04$ \\
\hline NGC 4254 Enuc. $1 \mathrm{c}$ & 121850.194 & +142418.6 & $0.35 \pm 0.01$ & $0.12 \pm 0.01$ & $0.18 \pm 0.01$ & 489.0 & 3.116 & $-0.41 \pm 0.03$ & $0.77 \pm 0.03$ \\
\hline NGC $4254 \mathrm{~d}$ & 121851.63 & +142508.599 & $0.30 \pm 0.01$ & $0.04 \pm 0.01$ & $0.12 \pm 0.02$ & 489.0 & & $-0.52 \pm 0.05$ & $0.64 \pm 0.07$ \\
\hline NGC $4254 \mathrm{e}$ & 121851.899 & +142449.699 & $0.08 \pm 0.01$ & $0.02 \pm 0.01$ & $0.13 \pm 0.02$ & 489.0 & 2.813 & $0.17 \pm 0.08$ & $1.00 \pm 0.02$ \\
\hline NGC $4254 \mathrm{f}$ & 121851.919 & +142440.099 & $0.04 \pm 0.01$ & $<0.02$ & $0.14 \pm 0.02$ & 489.0 & 3.149 & $0.55 \pm 0.14$ & $1.00 \pm 0.01$ \\
\hline NGC 4321 Enuc. 2 a & 122248.844 & +155012.8 & $0.06 \pm 0.01$ & $0.05 \pm 0.01$ & $0.05 \pm 0.01$ & 485.0 & 8.305 & $-0.10 \pm 0.10$ & $1.00 \pm 0.05$ \\
\hline NGC 4321 Enuc. $2 \mathrm{~b}$ & 122249.904 & +155027.8 & $0.06 \pm 0.01$ & $0.03 \pm 0.01$ & $0.05 \pm 0.01$ & 485.0 & 7.979 & $-0.13 \pm 0.10$ & $0.98 \pm 0.05$ \\
\hline NGC 4321 Enuc. 2 & 122250.652 & +155027.2 & $<0.03$ & $<0.02$ & $0.04 \pm 0.01$ & 485.0 & 7.285 & $\cdots$ & $\cdots$ \\
\hline NGC $4321 \mathrm{a}$ & 122254.651 & +154919.8 & $2.03 \pm 0.06$ & $0.81 \pm 0.03$ & $0.46 \pm 0.02$ & 485.0 & 0.284 & $-0.61 \pm 0.02$ & $0.50 \pm 0.03$ \\
\hline NGC $4321 \mathrm{~b}$ & 122255.129 & +154920.4 & $3.01 \pm 0.09$ & $1.07 \pm 0.03$ & $0.64 \pm 0.02$ & 485.0 & 0.270 & $-0.64 \pm 0.02$ & $0.44 \pm 0.03$ \\
\hline NGC 4321 Enuc. 1 & 122258.9 & +154935.003 & $<0.03$ & $<0.02$ & $<0.02$ & 485.0 & 4.520 & $\ldots$ & $\cdots$ \\
\hline NGC 4536 & 123427.06 & +021118.2 & $21.45 \pm 0.64$ & $7.81 \pm 0.23$ & $4.90 \pm 0.15$ & 492.0 & 0.126 & $-0.62 \pm 0.02$ & $0.48 \pm 0.03$ \\
\hline NGC 4559 a & 123556.273 & +275740.5 & $0.08 \pm 0.01$ & $0.07 \pm 0.01$ & $0.08 \pm 0.01$ & 237.0 & & $-0.01 \pm 0.08$ & $1.00 \pm 0.03$ \\
\hline NGC $4559 \mathrm{~b}$ & 123556.455 & +27572 & & & & & & $0.05 \pm 0$ & $1.00 \pm 0.04$ \\
\hline NGC $4559 \mathrm{c}$ & 123558.47 & +275729.7 & $0.10 \pm 0.01$ & $0.10 \pm 0.01$ & $0.11 \pm 0.01$ & 237.0 & 8 & $0.02 \pm 0.06$ & $1.00 \pm 0.02$ \\
\hline NGC 4569 & 123649.8 & +130946.6 & $2.99 \pm 0.09$ & $0.92 \pm 0.03$ & $52 \pm 0.02$ & 335.0 & & $-0.73 \pm 0.02$ & $0.26 \pm 0.04$ \\
\hline NGC 4579 & 123743.518 & +114905.6 & $21.36 \pm 0.64$ & $24.39 \pm 0.73$ & $30.71 \pm 0.92$ & 557.0 & 5 & $0.14 \pm 0.02$ & $1.00 \pm 0.01$ \\
\hline NGC 4594 a & 123959.42 & -113723 & $38.94 \pm 1.17$ & $45.37 \pm 1.36$ & $36.58 \pm 1.10$ & 308.0 & 0.052 & $-0.01 \pm 0.02$ & $1.00 \pm 0.01$ \\
\hline NGC 4631 Enuc. 1 & 124140.468 & +323149.1 & $0.16 \pm 0.01$ & $0.16 \pm 0.01$ & $0.14 \pm 0.01$ & 259.0 & & $-0.04 \pm 0.04$ & $1.00 \pm 0.02$ \\
\hline NGC 4625 & 124152.4 & +411624 & $<0.03$ & $0.04 \pm 0.01$ & $2 \pm 0.01$ & 316.0 & 0.139 & $-0.91 \pm 0.39$ & $0.24 \pm 0.82$ \\
\hline NGC 4631 a & 124203.433 & +323217.198 & $3.50 \pm 0.11$ & $1.05 \pm 0.03$ & $0.72 \pm 0.03$ & 259.0 & 3.189 & $-0.68 \pm 0.02$ & $0.37 \pm 0.04$ \\
\hline NGC $4631 \mathrm{~b}$ & 124203.978 & +32 3215.999 & $1.62 \pm 0.05$ & $1.06 \pm 0.03$ & $0.58 \pm 0$ & 259.0 & 3.435 & $-0.39 \pm 0.02$ & $0.79 \pm 0.02$ \\
\hline NGC $4631 \mathrm{c}$ & 124204.31 & +323225.299 & $2.35 \pm 0.07$ & $1.36 \pm 0.04$ & $1.29 \pm 0.04$ & 259.0 & 1.734 & $-0.26 \pm 0.02$ & $0.90 \pm 0.01$ \\
\hline NGC $4631 \mathrm{~d}$ & 124205.093 & +323210.6 & $0.36 \pm 0.01$ & $0.27 \pm 0.01$ & $0.26 \pm 0.01$ & 259.0 & 4.988 & $-0.14 \pm 0.03$ & $0.98 \pm 0.01$ \\
\hline NGC $4631 \mathrm{e}$ & 124205.568 & +323229.5 & $1 \pm 0.03$ & $0.44 \pm 0$ & 0. & 259.0 & & $-0.20 \pm 0$ & $0.94 \pm 0.01$ \\
\hline NGC 4631 En & 124221.988 & +32 & a & & & & & $-0.14=$ & $0.98 \pm 0.01$ \\
\hline NGC 4725 a & 125026.556 & +253003 & \pm 0.01 & $0.10 \pm 0$ & $6 \pm($ & 4.0 & & $0.50 \pm 0$ & $1.00 \pm 0.01$ \\
\hline NGC 4736 & 125053.053 & +410712.8 & \pm 0.12 & & 3 & 158.0 & & $5 \pm$ & $0.59 \pm 0.03$ \\
\hline NGC 4736 Enuc. 1 a & 125056.412 & +410714.3 & \pm 0.02 & 3 & 22 & 158.0 & & $-0.29 \pm$ & $0.88 \pm 0.02$ \\
\hline NGC 4736 Enuc. $1 \mathrm{~b}$ & 125056.704 & +410705 & $1.08 \pm 0.03$ & $0.62 \pm 0.03$ & $0.39 \pm 0$ & 158.0 & 0.939 & $-0.41 \pm 0.02$ & $0.77 \pm 0.02$ \\
\hline NGC 4736 Enuc. $1 \mathrm{c}$ & 125056.784 & +410647.6 & $0.64 \pm 0.02$ & $0.46 \pm 0.03$ & $0.26 \pm 0.02$ & 158.0 & & $-0.31 \pm$ & $0.86 \pm 0.03$ \\
\hline NGC 4826 & 125643.556 & +214 & \pm 0.13 & 05 & 03 & 179.0 & & $-0.62 \pm$ & $0.48 \pm 0.03$ \\
\hline NGC 5055 & 131549.308 & +420145.1 & $0.71 \pm 0.02$ & $0.35 \pm 0.01$ & 01 & 269.0 & 0.009 & $-0.55 \pm 0.03$ & $0.59 \pm 0.04$ \\
\hline NGC 5055 Enuc. 1 & 131558.323 & +4200 27.4 & $0.18 \pm 0.01$ & $5 \pm 0.01$ & 01 & 269.0 & 5.630 & $-0.07 \pm 0.04$ & $1.00 \pm 0.02$ \\
\hline NGC 5194 Enuc. 6 a & 132939.324 & +470840.7 & $0.26 \pm 0.02$ & $0.20 \pm 0.01$ & $0.21 \pm 0.01$ & 259.0 & 12.321 & $-0.09 \pm 0.03$ & $1.00 \pm 0.01$ \\
\hline NGC 5194 Enuc. 3 & 132945.13 & +470957.4 & $0.33 \pm 0.02$ & $0.25 \pm 0.01$ & $0.26 \pm 0.01$ & 259.0 & & $-0.10 \pm 0.03$ & $1.00 \pm 0.01$ \\
\hline NGC 5194 Enuc. 11 a & 132947.138 & +471341.298 & $0.26 \pm 0.02$ & $0.06 \pm 0.01$ & $0.10 \pm 0.01$ & & & $-0.42 \pm 0.04$ & $0.75 \pm 0.05$ \\
\hline NGC 5194 Enuc. 11 b & 132947.58 & +471324.799 & $<0.04$ & & $0.09 \pm 0$ & & & $-0.25 \pm 0$ & $0.91 \pm 0.12$ \\
\hline NGC 5194 Enuc. $11 \mathrm{~d}$ & 132949.582 & +471328.7 & $<0.04$ & +4 & $07 \pm 0$ & 259.0 & & $0.74 \pm 0$ & $1.00 \pm 0.02$ \\
\hline NGC 5194 Enuc. $11 \mathrm{c}$ & 132949.671 & +471400.2 & $42 \pm 0.02$ & $4+0$ & $6 \pm 0$ & 259.0 & 5.2 & $-0.75 \pm 0.05$ & $0.22 \pm 0.11$ \\
\hline NGC 5194c & 132950.022 & +471131.898 & \pm 0.03 & \pm 0 & $5 \pm 0$ & 259.0 & 1.8 & $-0.48 \pm$ & $0.69 \pm 0.04$ \\
\hline NGC 5194 Enuc. $11 \mathrm{e}$ & 132950.642 & +471344.9 & $0.20 \pm 0.01$ & $0.18 \pm 0.01$ & $0.13 \pm 0.01$ & 259.0 & 4.633 & $-0.18 \pm 0.04$ & $0.96 \pm 0.02$ \\
\hline NGC 5194 b & 132951.64 & +471206.7 & $1.09 \pm 0.04$ & $0.41 \pm 0.02$ & $0.22 \pm 0.01$ & 259.0 & 0.977 & $-0.64 \pm 0.02$ & $0.44 \pm 0.04$ \\
\hline NGC $5194 \mathrm{e}$ & 132952.553 & +471152.6 & $3.17 \pm 0.10$ & $1.13 \pm 0.04$ & $0.46 \pm 0.02$ & 259.0 & 0.365 & $-0.77 \pm 0.02$ & $0.17 \pm 0.05$ \\
\hline NGC 5194d & 132952.729 & +471140.6 & $4.68 \pm 0.14$ & $1.24 \pm 0.04$ & $0.53 \pm 0.02$ & 259.0 & 0.092 & $-0.89 \pm 0.02$ & $0.24 \pm 0.04$ \\
\hline NGC 5194 Enuc. $1 \mathrm{c}$ & 132953.129 & +471239.4 & $0.18 \pm 0.02$ & $0.06 \pm 0.01$ & $0.14 \pm 0.01$ & 259.0 & 2.323 & $-0.13 \pm 0.04$ & $0.98 \pm 0.02$ \\
\hline NGC 5194 Enuc. 4 a & 132953.932 & +471404.899 & $<0.04$ & $<0.02$ & $0.08 \pm 0.01$ & 259.0 & 5.880 & $\ldots$ & $\cdots$ \\
\hline NGC 5194 Enuc. 10 a & 132955.346 & +471047.199 & $12 \pm 0.02$ & $07 \pm 0.01$ & $0.09 \pm 0.01$ & 259.0 & 2.336 & $-0.16 \pm$ & $0.97 \pm 0.04$ \\
\hline NGC 5194 Enuc. $4 \mathrm{c}$ & 132955.611 & +471350.2 & $6 \pm 0.01$ & $06 \pm 0.01$ & $0.09 \pm 0.01$ & 259.0 & 8 & $9 \pm$ & $0.88 \pm 0.04$ \\
\hline NGC 5194 a & 132955.791 & +471145.097 & & & & & & -0 & $0.93 \pm 0.02$ \\
\hline NGC 5194 Enuc. $10 \mathrm{~b}$ & 132956.523 & +471046.9 & $5 \pm 0.02$ & $13 \pm($ & $0.14 \pm 0$ & 259.0 & 2.723 & $-0.24 \pm$ & $0.91 \pm 0.03$ \\
\hline NGC 5194 Enuc. 4 d & 132958.733 & +471409.398 & $0.13 \pm 0.01$ & $.08 \pm 0.01$ & $0.07 \pm 0.01$ & 259.0 & 7.696 & $-0.28 \pm 0.06$ & $0.89 \pm 0.05$ \\
\hline NGC 5194 Enuc. 5 a & 132959.6 & +471359.8 & $0.26 \pm 0.02$ & $.15 \pm 0.01$ & $0.17 \pm 0.01$ & 259.0 & 7.742 & $-0.20 \pm 0.04$ & $0.94 \pm 0.02$ \\
\hline NGC 5194 Enuc. 9 & 132959.782 & +4711 12.3 & $0.19 \pm 0.02$ & $0.17 \pm 0.01$ & $0.21 \pm 0.01$ & 259.0 & 4.131 & $0.03 \pm 0.04$ & $1.00 \pm 0.02$ \\
\hline NGC 5194 Enuc. 7 a & 133001.029 & +470928.599 & $0.07 \pm 0.02$ & $0.11 \pm 0.01$ & $0.12 \pm 0.02$ & 259.0 & 6.221 & $0.22 \pm 0.11$ & $1.00 \pm 0.03$ \\
\hline NGC 5194 Enuc. 8 & 133001.482 & +471251.7 & $0.46 \pm 0.02$ & $0.38 \pm 0.01$ & $0.29 \pm 0.01$ & 259.0 & 6.650 & $-0.17 \pm 0.03$ & $0.96 \pm 0.01$ \\
\hline NGC 5194 Enuc. 7 b & 133002.382 & +470948.7 & $0.45 \pm 0.02$ & $0.21 \pm 0.01$ & $0.20 \pm 0.01$ & 259.0 & 6.329 & $-0.38 \pm 0.03$ & $0.80 \pm 0.03$ \\
\hline NGC 5194 Enuc. 7 c & 133003.471 & +470940.3 & $0.18 \pm 0.02$ & $0.07 \pm 0.01$ & $0.05 \pm 0.01$ & 259.0 & 6.962 & $-0.55 \pm 0.08$ & $0.59 \pm 0.11$ \\
\hline NGC 5398 & 140120.105 & -330409.2 & $0.86 \pm 0.03$ & $0.62 \pm 0.02$ & $0.64 \pm 0.02$ & 260.0 & 1.381 & $-0.13 \pm 0.02$ & $0.98 \pm 0.01$ \\
\hline NGC 5457 Enuc. 6 a & 140228.203 & +541627.2 & $0.68 \pm 0.02$ & $0.39 \pm 0.01$ & $0.40 \pm 0.01$ & 227.0 & 15.707 & $-0.24 \pm 0.02$ & $0.91 \pm 0.01$ \\
\hline NGC 5457 Enuc. $6 \mathrm{~b}$ & 140229.607 & +541615.799 & $1.02 \pm 0.03$ & $0.56 \pm 0.02$ & $0.45 \pm 0.02$ & 227.0 & 15.550 & $-0.34 \pm 0.02$ & $0.83 \pm 0.02$ \\
\hline NGC 5457 Enuc. $6 \mathrm{c}$ & 140230.566 & +541609.798 & $0.58 \pm 0.02$ & $0.42 \pm 0.02$ & $0.30 \pm 0.02$ & 227.0 & 15.422 & $-0.25 \pm 0.02$ & $0.91 \pm 0.02$ \\
\hline
\end{tabular}


Table 5

(Continued)

\begin{tabular}{|c|c|c|c|c|c|c|c|c|c|}
\hline Source ID & $\begin{array}{l}\text { R.A. } \\
\text { (J2000) }\end{array}$ & $\begin{array}{c}\text { Decl. } \\
(\mathrm{J} 2000)\end{array}$ & $\begin{array}{l}S_{3 \mathrm{GHz}} \\
(\mathrm{mJy})\end{array}$ & $\begin{array}{c}S_{15 \mathrm{GHz}} \\
(\mathrm{mJy})\end{array}$ & $\begin{array}{c}S_{33 \mathrm{GHz}} \\
(\mathrm{mJy})\end{array}$ & $\begin{array}{c}d_{\mathrm{ap}} \\
(\mathrm{pc})\end{array}$ & $\begin{array}{c}r_{\mathrm{G}} \\
(\mathrm{kpc})\end{array}$ & $\alpha$ & $f_{\mathrm{T}}^{33 \mathrm{GHz}}$ \\
\hline NGC 5457 Enuc. 5 a & 140301.203 & +541428.4 & $0.96 \pm 0.03$ & $0.68 \pm 0.02$ & $0.72 \pm 0.02$ & 227.0 & 13.129 & $-0.14 \pm 0.02$ & $0.98 \pm 0.01$ \\
\hline NGC 5457 Enuc. 1 & 140310.2 & +542057.8 & $08 \pm 0.01$ & $<0.02$ & $0.05 \pm 0.01$ & 227.0 & 0.744 & $-0.15 \pm 0.07$ & $0.97 \pm 0.04$ \\
\hline NGC 5457 & 140312.531 & +542055.2 & $34 \pm 0.01$ & $0.09 \pm 0.01$ & $0.15 \pm 0.01$ & 227.0 & & $-0.41 \pm 0.03$ & $0.77 \pm 0.03$ \\
\hline NGC 5457 Enuc. 3 a & 140338.317 & +541851.398 & $30 \pm 0.02$ & $7 \pm 0.02$ & $<0.10$ & 227.0 & & & $0.82 \pm 0.09$ \\
\hline NGC 5457 Enuc. 3 b & 140339.894 & +541856.799 & $48 \pm 0.02$ & $4 \pm 0.03$ & $.35 \pm 0.03$ & 227.0 & 9.655 & $-0.10 \pm 0.03$ & $1.00 \pm 0.02$ \\
\hline NGC 5457 Enuc. $3 \mathrm{c}$ & 140341.437 & +541904.9 & $27 \pm 0.13$ & $33 \pm 0.10$ & $91 \pm 0.09$ & 227.0 & 9.964 & $-0.16 \pm 0.02$ & $0.97 \pm 0.01$ \\
\hline NGC 5457 Enuc. $3 \mathrm{~d}$ & 140342.912 & +541924.699 & $0.42 \pm 0.02$ & $.24 \pm 0.02$ & $.21 \pm 0.03$ & 227.0 & 10.113 & $-0.31 \pm 0.05$ & $0.86 \pm 0.04$ \\
\hline NGC 5457 Enuc. 4 a & 140352.036 & +542152.5 & $0.18 \pm 0.01$ & $0.12 \pm 0.01$ & $.10 \pm 0.01$ & 227.0 & 12.149 & $-0.24 \pm 0.04$ & $0.91 \pm 0.03$ \\
\hline NGC 5457 Enuc. 4 b & 140352.997 & +542157.3 & $0.20 \pm 0.01$ & $0.15 \pm 0.01$ & $0.14 \pm 0.01$ & 227.0 & 12.453 & $-0.16 \pm 0.04$ & $0.97 \pm 0.02$ \\
\hline NGC 5457 Enuc. $4 \mathrm{c}$ & 140353.203 & +542206.3 & $0.46 \pm 0.02$ & $0.26 \pm 0.01$ & $0.28 \pm 0.01$ & 227.0 & 12.540 & $-0.22 \pm 0.02$ & $0.93 \pm 0.01$ \\
\hline NGC 5457 Enuc. $4 \mathrm{~d}$ & 140353.993 & +542210.8 & $0.50 \pm 0.02$ & $0.26 \pm 0.01$ & $0.25 \pm 0.01$ & 227.0 & 12.795 & $-0.32 \pm 0.02$ & $0.86 \pm 0.02$ \\
\hline NGC 5474 & 140501.3 & +533944 & $<0.03$ & $<0.02$ & $<0.02$ & 231.0 & 0.070 & $\ldots$ & $\cdots$ \\
\hline NGC 5713 Enuc. 2 a & 144010.8 & -001735.5 & $0.82 \pm 0.03$ & $0.48 \pm 0.02$ & $0.26 \pm 0.01$ & 726.0 & 1.984 & $-0.44 \pm 0.02$ & $0.74 \pm 0.03$ \\
\hline NGC 5713 Enuc. 2 b & 144010.86 & -001750.2 & $0.41 \pm 0.02$ & $0.27 \pm 0.02$ & $0.09 \pm 0.01$ & 726.0 & 5 & $-0.42 \pm$ & $0.75 \pm 0.04$ \\
\hline NGC 5713 & 144011.3 & -001727 & 0.04 & & 0 & 726.0 & & $-0.52=$ & $0.63 \pm 0.03$ \\
\hline NGC 5713 Enuc. 1 & 144011.36 & -001718.2 & $4 \pm 0.17$ & $2.28 \pm$ & 1 & 726.0 & & $6 \pm$ & $0.57 \pm 0.03$ \\
\hline NGC 5866 & 1506 & .7 & . & $7-$ & & 519.0 & & -0 & $0.78 \pm 0.02$ \\
\hline NGC 6946 Enuc. $4 \mathrm{c}$ & 3422.738 & +601034.197 & $0.68 \pm$ & $0.70=$ & 2 & 231.0 & 8.7 & $-0.03 \pm$ & $1.00 \pm 0.01$ \\
\hline NGC 6946 Enuc. 8 & 203432.28 & +601019.3 & $1.16 \pm 0.04$ & $0.56=$ & 0.58 & 231.0 & 6.122 & $-0.32 \pm 0.02$ & $0.85 \pm 0.02$ \\
\hline NGC 6946 Enuc. 5 a & 20343 & +600510.099 & $<0.03$ & $<0.02$ & $007+$ & 231.0 & 9.239 & $\ldots$ & $\ldots$ \\
\hline NGC 6946 Enuc. 5 b & 203439.361 & +600452.4 & $0.14 \pm 0.01$ & $0.16 \pm 0.01$ & $0.16 \pm 0.01$ & 231.0 & 9.704 & $0.06 \pm 0.04$ & $1.00 \pm 0.01$ \\
\hline NGC 6946 Enuc. 3 a & 203449.865 & +601240.699 & $0.04 \pm 0.01$ & $0.11 \pm 0.01$ & $0.10 \pm 0.01$ & 231.0 & 7.742 & $0.18 \pm 0.09$ & $1.00 \pm 0.02$ \\
\hline NGC 6946 Enuc. 3 b & 203452.24 & +601243.7 & $0.19 \pm 0.01$ & $0.18 \pm 0.01$ & $0.17 \pm 0.01$ & 231.0 & 7.729 & $-0.04 \pm 0.03$ & $1.00 \pm 0.01$ \\
\hline NGC 6946 b & 203452.26 & +600914.3 & $19.95 \pm 0.60$ & $8.56 \pm 0.26$ & $5.55 \pm 0.17$ & 231.0 & 0.016 & $-0.53 \pm 0.02$ & $0.62 \pm 0.03$ \\
\hline NGC 6946 Enuc. 6 b & 203506.965 & +601046.5 & $0.21 \pm 0.01$ & $0.19 \pm 0.01$ & $0.12 \pm 0.01$ & 231.0 & 4.728 & $-0.14 \pm 0.04$ & $0.98 \pm 0.02$ \\
\hline NGC 6946 Enuc. 9 & 203511.086 & +600857.45 & $0.83 \pm 0.03$ & $0.73 \pm 0.02$ & $0.71 \pm 0.02$ & 231.0 & 5.071 & $-0.07 \pm 0.02$ & $1.00 \pm 0.01$ \\
\hline NGC 6946 Enuc. 7 & 203512.974 & +600850.55 & & & & & & $0.13 \pm 0.03$ & $1.00 \pm 0.01$ \\
\hline NGC 6946 Enuc. 1 & 20351 & & & & & & & & \\
\hline NGC 7331 & 223704.1 & & & & & & 0.0 & 0 . & $1.00 \pm 0.01$ \\
\hline NGC 7793 Enuc. 1 & 235748.8 & -323657.991 & $0.05 \pm 0.01$ & $0.02 \pm 0.01$ & $<0.07$ & 133.0 & 2.574 & $-0.42 \pm 0.25$ & $0.76 \pm 0.27$ \\
\hline NGC 7793 Enuc. 3 & 235748.8 & -323451.995 & $0.10 \pm 0.01$ & $0.10 \pm 0.01$ & $<0.08$ & 133.0 & 1.016 & $-0.03 \pm 0.09$ & $1.00 \pm 0.04$ \\
\hline NGC 7793 a & 235749.2 & -323524 & $0.06 \pm 0.01$ & $0.04 \pm 0.01$ & $0.05 \pm 0.01$ & 133.0 & 0.174 & $-0.18 \pm 0.14$ & $0.95 \pm 0.09$ \\
\hline NGC 7793 b & 235749.58 & -323525.6 & $0.10 \pm 0.01$ & $0.05 \pm 0.01$ & $0.07 \pm 0.01$ & 133.0 & 0.081 & $-0.26 \pm 0.09$ & $0.90 \pm 0.07$ \\
\hline NGC 7793 Enuc. 2 & 235756.1 & -323540 & $<0.04$ & $<0.02$ & $<0.04$ & 133.0 & 1.526 & $\ldots$ & $\ldots$ \\
\hline
\end{tabular}

Likely Associated with Supernovae

\begin{tabular}{|c|c|c|c|c|c|c|c|c|c|}
\hline GC 6946 Enuc. 6 a & 203506.08 & +601058.5 & $0.72 \pm 0.02$ & $0.42 \pm 0.01$ & $0.48 \pm 0.02$ & 231.0 & 4.855 & $-0.20 \pm 0.02$ & $0.94 \pm 0.01$ \\
\hline \multicolumn{10}{|c|}{ Likely AME Candidates } \\
\hline GC 2403 Enuc. 5 & 073619.839 & +653705.5 & $0.64 \pm 0.02$ & $0.59 \pm 0.02$ & $0.56 \pm 0.02$ & 109.0 & 3.464 & $-0.06 \pm 0.02$ & $1.00 \pm 0.01$ \\
\hline NGC 2403 Enuc. 3 & 948 & 3639 & $1 \pm 0.06$ & $3 \pm 1$ & 5 & 9.0 & & 02 & 0.01 \\
\hline NGC 4254 Enuc. 1 a & 121849.203 & +142357.9 & $6 \pm 0.01$ & $0.07 \pm 0.01$ & $2 \pm 0.01$ & 489.0 & 4.428 & $-0.18 \pm 0.04$ & $0.95 \pm 0.03$ \\
\hline NGC $4631 \mathrm{f}$ & 124206.256 & +323231.9 & $0.79 \pm 0.03$ & $0.27 \pm 0.01$ & $1 \pm 0.02$ & 259.0 & 1.566 & $-0.23 \pm 0.02$ & $0.92 \pm 0.01$ \\
\hline NGC $4631 \mathrm{~g}$ & 124207.466 & +32 3231.599 & $1.65 \pm 0.05$ & $0.88 \pm 0.03$ & $7 \pm 0.03$ & 259.0 & 0.960 & $-0.25 \pm 0.02$ & $0.91 \pm 0.01$ \\
\hline NGC 4631 h & 124208.059 & +323234.899 & $2.07 \pm 0.06$ & $0.97 \pm 0.03$ & $1.29 \pm 0.04$ & 259.0 & 1.769 & $-0.24 \pm 0.02$ & $0.92 \pm 0.01$ \\
\hline NGC 4631 Enı & 124221.419 & +323306.3 & $0.28 \pm 0.01$ & $0.11 \pm 0.01$ & $8 \pm 0.01$ & 259.0 & 9.974 & $-0.22 \pm 0.03$ & $0.93 \pm 0.02$ \\
\hline NGC $4725 \mathrm{~b}$ & 125028.484 & +253021.899 & $8+0.01$ & $0.11 \pm 0.01$ & $9 \pm 0.01$ & 404.0 & 1.921 & \pm 0.06 & $=0.01$ \\
\hline NGC 5194 Enuc. 2 & 132944.1 & +471023.4 & $0.66 \pm 0.02$ & $0.37 \pm 0.01$ & $9 \pm 0.01$ & 259.0 & 6.834 & $-0.24 \pm 0.02$ & $0.92 \pm 0.01$ \\
\hline NGC 5194 Enuc. 1 a & 132949.508 & +471240.296 & $9 \pm 0.02$ & 4 & 0.02 & 259.0 & 2.534 & $0.09 \pm 0.03$ & 0.01 \\
\hline NGC 5194 Enuc. 1 b & 1329 & +471243.6 & $0.28 \pm 0.02$ & $0.24=$ & $7 \pm 0.01$ & 259.0 & 2.322 & $-0.02 \pm 0.03$ & 0.01 \\
\hline NGC $5194 \mathrm{E}$ & 1329 & 1.6 & $5 \pm 0$. & 0.13 & 01 & 259.0 & 6.193 & -0 & 0.02 \\
\hline NGC 5457 Enuc. 2 & 1402 & +542227.5 & $8 \pm 0.01$ & $0.11=$ & 01 & 227.0 & 6.451 & -0 & $1.00 \pm 0.02$ \\
\hline NGC 5457 Enuc. 7 & 140429.334 & +542347.6 & $2.15 \pm 0.07$ & $1.07 \pm 0.03$ & $1.14 \pm 0.03$ & 227.0 & 23.861 & $-0.29 \pm 0.02$ & $0.88 \pm 0.01$ \\
\hline NGC 6946 Enuc. 4 a & 203419.842 & +601006.6 & $0.92 \pm 0.03$ & $0.75 \pm 0.02$ & $0.83 \pm 0.03$ & 231.0 & 9.044 & $-0.06 \pm 0.02$ & $1.00 \pm 0.01$ \\
\hline NGC 6946 Enuc. 4 b & 203421.41 & +60 1017.699 & $0.06 \pm 0.01$ & $0.42 \pm 0.01$ & $0.34 \pm 0.01$ & 231.0 & 8.801 & $0.13 \pm 0.05$ & $1.00 \pm 0.01$ \\
\hline NGC 6946 a & 203451.295 & +600939.2 & $0.35 \pm 0.02$ & $0.35 \pm 0.01$ & $0.51 \pm 0.03$ & 231.0 & 1.004 & $0.09 \pm 0.03$ & $1.00 \pm 0.01$ \\
\hline NGC 6946 c & 203452.742 & +600930.5 & $0.27 \pm 0.01$ & $0.34 \pm 0.01$ & $0.25 \pm 0.03$ & 231.0 & 0.601 & $0.08 \pm 0.04$ & $1.00 \pm 0.01$ \\
\hline NGC 6946 Enuc. 2 a & 203523.572 & +600948.899 & $0.05 \pm 0.01$ & $0.16 \pm 0.01$ & $0.15 \pm 0.01$ & 231.0 & 8.120 & $0.30 \pm 0.08$ & $1.00 \pm 0.01$ \\
\hline NGC 6946 Enuc. 2 b & 203525.381 & +600958.8 & $1.03 \pm 0.03$ & $0.76 \pm 0.02$ & $0.70 \pm 0.02$ & 231.0 & 8.595 & $-0.17 \pm 0.02$ & $0.96 \pm 0.01$ \\
\hline
\end{tabular}


multiplied by the effective area of the beam to get an estimate of the local diffuse background emission. These values are given in Table A2. Further, we measure the fractional contribution of the background emission for each region and find that the median value is $4.7 \% \pm 0.43 \%, 6.6 \% \pm 0.79 \%$, and $3.9 \% \pm 0.71 \%$ with median absolute deviations of $5.3 \%$, $5.4 \%$, and $4.5 \%$ for our 3,15 , and $33 \mathrm{GHz}$ observations, respectively. Importantly, these values are smaller than the $15 \%-40 \%$ found for the regions studied at $25^{\prime \prime}(\sim 1 \mathrm{kpc})$ scales with the GBT (Murphy et al. 2011).

The regions, listed in Tables 4 and 5, are named according to the nearest $33 \mathrm{GHz}$ image, with an alphabetical suffix if there are multiple regions corresponding to one image. For example, "NGC 2403 Enuc. 2 B" is the second of three regions within the VLA pointing of extranuclear region 2 in NGC 2403. We distinguish individual sources identified in the 7 " smoothed maps by instead using a lowercase letter. For example, "NGC 2403 Enuc. 2 b" is one of two regions in the image of extranuclear region 2 in NGC 2403 and is composed of the sum contribution of "NGC 2403 Enuc. 2 B" and "NGC 2403 Enuc. $2 \mathrm{C}$ " in the full-resolution maps.

\section{Results}

Using the 3,15 , and $33 \mathrm{GHz}$ photometry, along with the $8 \mu \mathrm{m}$ imaging from Spitzer, we classify each region as either a star-forming region (SF), a background galaxy candidate (BG), a likely supernova remnant ( $\mathrm{SNe} / \mathrm{R}$; see Section 5.4$)$, or an AME candidate (see Section 5.5). In total we have identified 320 star-forming regions, 14 likely background galaxies, 10 likely supernovae/supernova remnants, and 33 AME candidates. Given that we are primarily interested in emission arising from our sample galaxies, the potential background galaxies have been removed from all plots and are discussed as a separate population of sources in Section 5.3. Regions identified at $7^{\prime \prime}$ that include emission from potential AME and $\mathrm{SNe} / \mathrm{R}$ candidates are correspondingly classified in Table 5.

We present results for the spectral index and thermal fraction distributions only including regions identified in the SFRS that have an $\mathrm{S} / \mathrm{N} \geqslant 3$ measured in at least two radio bands. This ensures that our fit results are not biased by single detections at one frequency and allows us to make accurate comparisons with the regions identified in M18a, for which a detection at $33 \mathrm{GHz}$ was required. This requirement removes 4 likely background galaxies, 4 likely supernova remnants, and 34 starforming regions. These single-band detections are distributed almost uniformly across all three frequency bands: 19, 11, and 12 at 3,15 , and $33 \mathrm{GHz}$, respectively. Accordingly, in the sample used to study the radio spectral indices and thermal fractions, we have retained 335 (286 SF, $10 \mathrm{BG}, 6 \mathrm{SNe} / \mathrm{R}$, and 33 AME) of the 377 sources with a statistically significant detection in at least one band. The median $\mathrm{S} / \mathrm{N}$ of these 335 sources is 18,15 , and 9 for detections at 3,15 , and $33 \mathrm{GHz}$, respectively.

We apply the same criteria for sources included in the spectral index and thermal fraction analysis using the $7^{\prime \prime}$ smoothed images. This removes 17 star-forming regions. These single-band detections are also distributed uniformly across all three bands: 7, 4, and 6 at 3,15, and $33 \mathrm{GHz}$, respectively. Accordingly, we have retained 163 (142 SF, 0 BG, $1 \mathrm{SNe} / \mathrm{R}$, and $20 \mathrm{AME}$ ) of the 180 sources with a statistically significant detection in at least one band. The median $\mathrm{S} / \mathrm{N}$ of these 163 sources is 24, 23, and 19 for detections at 3, 15, and $33 \mathrm{GHz}$, respectively.

\subsection{Spectral Indices}

The simplest approach to modeling the radio spectra of galaxies is by adopting a two-component power law, with the thermal/nonthermal ratio and the nonthermal spectral index allowed to vary as free parameters. For many star-forming galaxies in the local universe, this model adequately describes the dominant physical processes occurring at radio frequencies (Condon 1992). However, a robust interpretation of the radio spectrum can be complex. For example, the thermal and nonthermal fractions may vary with galaxy mass (e.g., Hughes et al. 2007; Bell 2003), the nonthermal index can vary within galaxies (Tabatabaei et al. 2017), and AME may add an additional component to the radio spectra at high frequencies in some regions (e.g., Murphy et al. 2010, 2018b).

To measure the $3-33 \mathrm{GHz}$ spectral indices, we performed a linear least-squares fit to the data with a single power law representing the combination of thermal and nonthermal emission. The distributions of the measured spectral slopes with the 10 likely background galaxies removed are given in the top panel of Figure 2 (325 total regions). The median spectral indices we measure for $3-15 \mathrm{GHz}, 15-33 \mathrm{GHz}$, and $3-33 \mathrm{GHz}$ are $-0.25 \pm 0.024,-0.20 \pm 0.043$, and $-0.23 \pm 0.018$, respectively. The median absolute deviation of these distributions is $0.34,0.61$, and 0.27 , respectively. Interestingly, we do not see particularly steep spectral indices from 3 to $15 \mathrm{GHz}$, indicating that the contribution of nonthermal emission to the radio flux density of individual star-forming regions is marginal on $\sim 100$ pc scales in these galaxies. This is consistent with the results presented in Murphy et al. (2012), where they show that the thermal fraction at $33 \mathrm{GHz}$ increased as a function of decreasing linear resolution. Despite the relatively flat spectral slopes from 3 to $15 \mathrm{GHz}$, we do see evidence that the spectrum continues to flatten from 15 to $33 \mathrm{GHz}$, on average. This is consistent with expectations for star-forming regions, where the radio spectrum is synchrotron dominated at low frequencies and flattens at higher frequencies as the contribution of thermal emission increases (Clemens et al. 2010; Condon et al. 2012; Murphy et al. 2013). Finally, we do not see any significant evidence for free-free absorption, which is known to affect the compact central regions of local star-forming and starburst galaxies and would result in steep spectral indices even at high frequencies (Condon \& Yin 1990; Clemens et al. 2008; Murphy et al. 2013).

In the nearby universe, the typical radio spectrum of normal star-forming galaxies is well described by a power-law spectrum with a spectral index of -0.7 and a thermal fraction of $\sim 10 \%$ at $\sim 1 \mathrm{GHz}$ (Klein et al. 1988; Condon \& Yin 1990), whereas studies of local luminous infrared galaxies (LIRGs) find a flat spectrum around $1 \mathrm{GHz}$ and a steepening spectrum above $10 \mathrm{GHz}$ (Clemens et al. 2008; Leroy et al. 2011; Murphy et al. 2013). New high-resolution observations of a large sample of LIRGs in the Great Observatories All-Sky LIRG Survey (GOALS) have revealed that the steep spectra seen in highly star-forming galaxies are attributed solely to the nucleus and that in extranuclear regions the spectral shape is typical of the star-forming regions identified in this study (Linden et al. 2019). 


\subsection{Thermal Fractions}

Given the results above, we now calculate the thermal fraction of each region by using the spectral index, measured in Section 4.1, from 3 to $15 \mathrm{GHz}\left(\alpha_{3-15 \mathrm{GHz}}\right)$ to set the lower limit on the nonthermal spectral index $\left(\alpha^{\mathrm{NT}}\right)$ such that $\alpha^{\mathrm{NT}}=-0.83$ if $\alpha_{3-15 \mathrm{GHz}} \geqslant-0.83$, and $\alpha^{\mathrm{NT}}=\alpha_{3-15 \mathrm{GHz}}$ if $\alpha_{3-15 \mathrm{GHz}}<$ -0.83 . A constant nonthermal radio spectral index of -0.83 is assumed based on the average nonthermal spectral index found among the 10 star-forming regions studied in NGC 6946 by Murphy et al. (2011). Furthermore, this value is consistent with the results of Niklas \& Beck (1997, i.e., $\alpha^{\mathrm{NT}}=-0.83$ with a scatter of $\sigma_{\alpha^{\mathrm{NT}}}=0.13$ ) for a sample of 74 nearby galaxies. Finally, we adopt a single power-law exponent for the free-free emission $(\sim-0.1)$ and use the fit from 3 to $33 \mathrm{GHz}$ to set the overall radio spectral index. Then, using the prescription in Klein et al. (1984), we can calculate the thermal fraction at $33 \mathrm{GHz}$ such that

$$
f_{\mathrm{T}}^{\nu_{1}}=\frac{\left(\frac{\nu_{2}}{\nu_{1}}\right)^{-\alpha}-\left(\frac{\nu_{2}}{\nu_{1}}\right)^{-\alpha^{\mathrm{NT}}}}{\left(\frac{\nu_{2}}{\nu_{1}}\right)^{-0.1}-\left(\frac{\nu_{2}}{\nu_{1}}\right)^{-\alpha^{\mathrm{NT}}}},
$$

where $\nu_{1}$ is the target frequency $(33 \mathrm{GHz}), \alpha$ is the observed slope from 3 to $33 \mathrm{GHz}$, and $\alpha^{\mathrm{NT}}$ is the nonthermal spectral index. In the top panel of Figure 3 we show the resulting thermal fractions of the star-forming regions in our sample using the empirically measured values from Equation (1). We find that the median value is $92 \% \pm 0.8 \%$ with a median absolute deviation of $11 \%$. This demonstrates that we can reliably use the $33 \mathrm{GHz}$ flux density to infer the total free-free emission, and thus current star formation activity, on the scales of individual $\mathrm{HII}$ and star-forming regions. While this result had been suggested by our previous GBT and VLA campaigns (e.g., Murphy et al. 2011, 2012, 2018a), this is the first measurement of the $33 \mathrm{GHz}$ thermal fraction based on the shape of the radio spectrum at these frequencies and spatial scales in nearby galaxies.

Further, by restricting our analysis such that we remove all non-SF regions and all apertures containing multiple smaller individual regions and require $r_{\mathrm{G}} \geqslant 250 \mathrm{pc}$ (238 regions), we mitigate contamination from any central AGN and can determine broad population statistics for extragalactic H II regions using our cleanest sample of extranuclear star-forming regions. In Figure 4 we find that the median thermal fraction is $93 \% \pm 0.8 \%$ with a median absolute deviation of $10 \%$. This value is consistent with the results presented in Figures 2 and 3 and confirms that supernova remnants, ultracompact $\mathrm{H}$ II regions, and/or AME candidates do not bias our results.

Finally, we measure the thermal fraction at $33 \mathrm{GHz}$ for 163 regions identified at 7 " resolution in M18a to be $94 \% \pm 0.8 \%$ with a median absolute deviation of $8 \%$. This result is consistent with the measurements at $2^{\prime \prime}$ and confirms that free-free emission dominates the radio spectra of star-forming regions on scales up to $\sim 500 \mathrm{pc}$. Further, when we apply the same radial cut (i.e., $r_{\mathrm{G}}>250 \mathrm{pc}$ ) and remove all non-SF regions, retaining $111 / 163$ regions, the median thermal fraction at $33 \mathrm{GHz}$ is $97 \% \pm 0.5 \%$ with a median absolute deviation of $4.6 \%$. Thus, it is clear that, regardless of the size of the photometric apertures used, isolating extranuclear SF regions in nearby galaxies results in a higher thermal fraction at $33 \mathrm{GHz}$ and a smaller median absolute deviation in the overall distribution.

\subsection{MCMC Parameter Estimation}

From Equation (1) it is clear that reliable estimates for the $33 \mathrm{GHz}$ thermal fraction are sensitive to the value adopted for the nonthermal spectral index $\left(\alpha^{\mathrm{NT}}\right)$. While the median $3-15 \mathrm{GHz}$ spectral index observed for our sample is well below the canonical value (i.e., -0.83 ), degeneracies may still exist between $\alpha^{\mathrm{NT}}$ and $f_{\mathrm{T}}$. In this case classical $\chi^{2}$ fitting methods may underestimate the true uncertainties associated with modeling the radio spectrum as a two-component power law (i.e., $S(\nu)=A \nu^{\alpha^{N T}}+B \nu^{-0.1}$ ). Here we explore whether the marginalized posterior distributions from a Monte Carlo Markov Chain (MCMC) analysis better reflect the uncertainties associated with this decomposition (Hogg et al. 2010).

For this exercise, we use the Python package EMCEE (Foreman-Mackey et al. 2013) to generate posterior probability distributions for each of the fitted parameters $\left(\alpha^{\mathrm{NT}}, f_{\mathrm{T}}\right.$, and $A / B$ ) given the typical $\mathrm{S} / \mathrm{N}$ of our three-band observations. Following Westcott et al. (2018), we parameterize our twocomponent power-law model at a reference frequency of $1 \mathrm{GHz}$ to avoid dependencies in frequency space. Further, we adopt a Gaussian probability distribution function for the nonthermal spectral index whose mean and standard deviation are consistent with the values obtained in Niklas \& Beck (1997). Finally, we make a slight modification to Equation (9) presented in Westcott et al. (2018),

$$
P(\theta) \propto H(A, B) e^{\frac{-(\alpha-0.83)^{2}}{0.13^{2}}},
$$

such that $\mathrm{H}$ is equal to 1 when the values of $A$ and $B$ are greater than zero. This is done in order to constrain the nonthermal spectral index, thermal fraction, and normalization constants of each component simultaneously. We make 1000 realizations of this model at three different $\mathrm{S} / \mathrm{Ns}(5,10$, and 50$)$ by randomly selecting a nonthermal spectral index $\left(-2<\alpha^{\mathrm{NT}}<0\right)$ and vales of $A(B)[0<A(B)<1]$, to represent the typical variance in the values observed for our sample.

In Figure 5 we plot the relative difference in the input and output thermal fraction as a function of the input nonthermal spectral index. For a fixed S/N per region of 10 , and with only three data points, our MCMC modeling can recover the input nonthermal spectral index to within $1 \sigma$ for $-1.25<\alpha^{\mathrm{NT}}<$ 0.25 . Importantly, we find that the best-constrained spectra have nonthermal indices very close to the fixed value adopted for our $\chi^{2}$-minimization $(-0.83)$. This result demonstrates that adopting fixed values for the $\alpha^{\mathrm{NT}}$ does not introduce systematic biases into the derived $33 \mathrm{GHz}$ thermal fractions, over a reasonable set of input conditions for our two-component power-law model. This is an important result for calibrations of the total SFR, which rely on using the observed radio continuum (Murphy et al. 2011, 2012).

\section{Discussion}

\subsection{Trends with Galactocentric Radius}

Following the same procedure as M18a, we use the measured P.A. and inclination of each galaxy to convert the angular separation of each star-forming region from the nucleus into a deprojected galactocentric radius $\left(r_{\mathrm{G}}\right)$. In M18a we found that the median $33 \mathrm{GHz}$ continuum-to- $\mathrm{H} \alpha$ line flux ratio was 

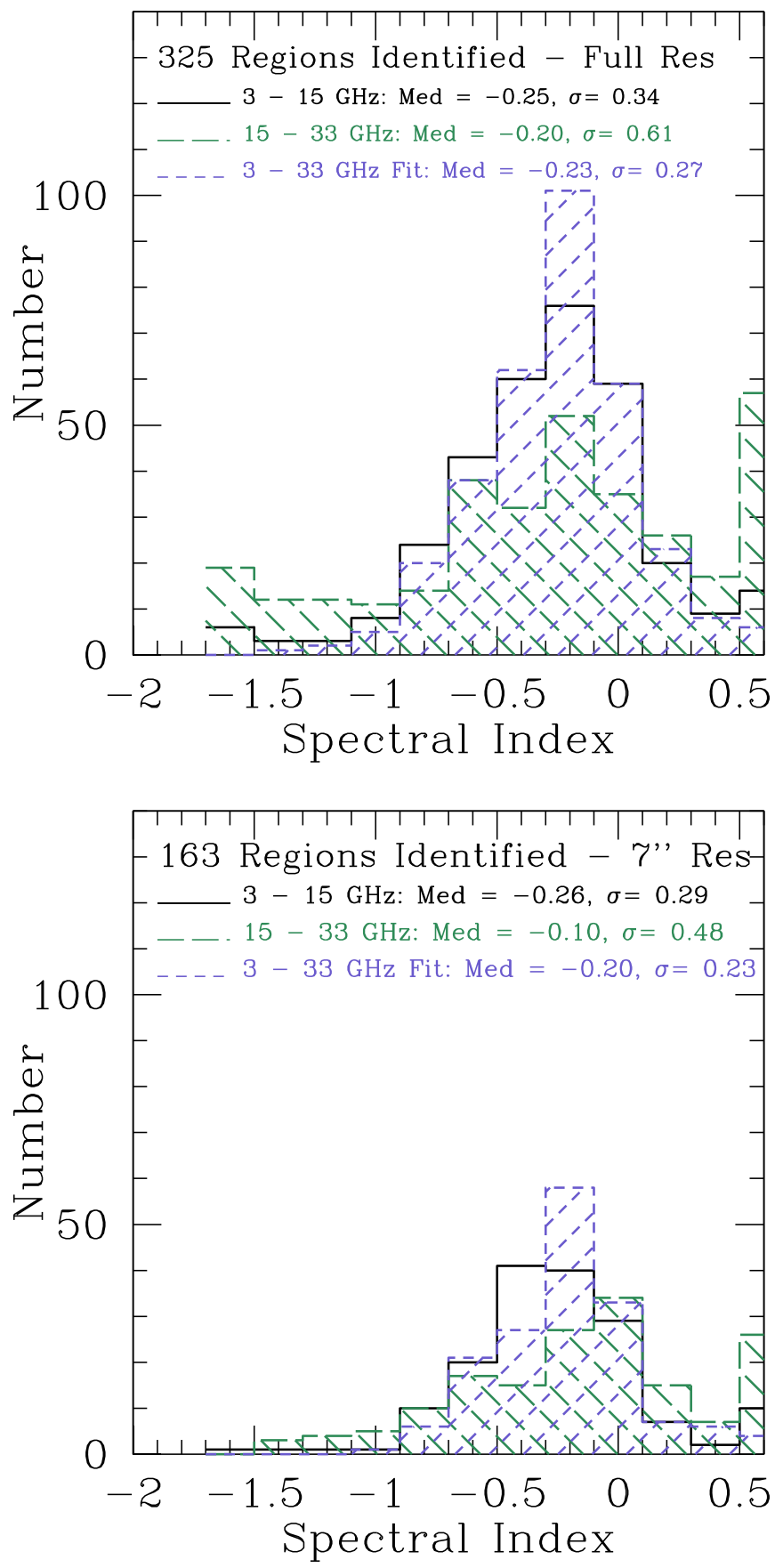

Figure 2. Top: 3-15 GHz (black), $15-33 \mathrm{GHz}$ (green), and 3-33 GHz (purple) radio spectral index distributions for 325 regions identified in the SFRS. The median size of the apertures used is $162 \pm 6.5 \mathrm{pc}$. Bottom: spectral index distributions for $1637^{\prime \prime}$ regions identified in M18a. The median size of the apertures used at $7^{\prime \prime}$ resolution is $259 \pm 7.2 \mathrm{pc}$. Overall, we find that the $3-33 \mathrm{GHz}$ distributions measured at $\sim 2^{\prime \prime}$ and $7^{\prime \prime}$ are consistent with one another, implying that free-free emission dominates the radio spectra of starforming regions on scales up to $\sim 500 \mathrm{pc}$. Not included in any of these plots are the likely background galaxy candidates identified.

statistically larger within $r_{\mathrm{G}}<250 \mathrm{pc}$ relative to the outer disk regions by a factor of $1.82 \pm 0.39$, while the ratio of $33 \mathrm{GHz}-$ $24 \mu \mathrm{m}$ flux densities is lower by a factor of $0.45 \pm 0.08$. Such a situation may arise if the circumnuclear regions of these galaxies have extended star formation histories (SFHs) in which star formation has taken place over a longer period of time, resulting in an accumulation of young dust-heating stars in addition to much older bulge stars that boost the $24 \mu \mathrm{m}$ flux
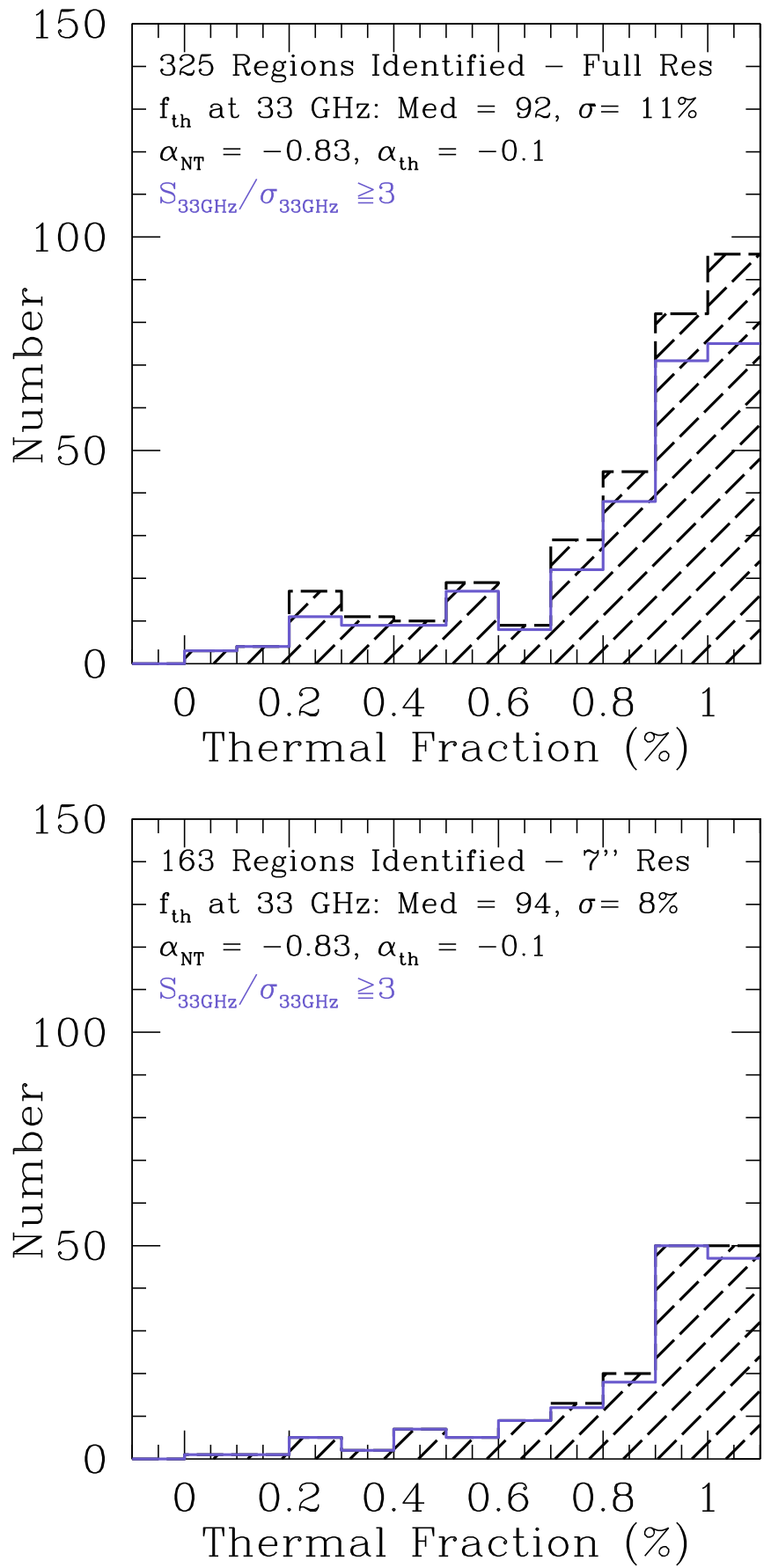

Figure 3. Top: thermal fraction distribution at $33 \mathrm{GHz}$ for 325 star-forming regions in the SFRS (black). In purple we show the distribution for regions with an $\mathrm{S} / \mathrm{N} \geqslant 3$ at $33 \mathrm{GHz}$, demonstrating that the lack of a significant $33 \mathrm{GHz}$ detection does not bias our results. The median size of the apertures used is $162 \pm 6.5 \mathrm{pc}$. Bottom: thermal fraction distribution for $1637^{\prime \prime}$ regions identified in M18a with an S/N > 3 in two radio bands. The median size of the apertures used at $7^{\prime \prime}$ resolution is $259 \pm 7.2 \mathrm{pc}$. Overall, we find that the median thermal fraction at $33 \mathrm{GHz}$ is $\sim 93 \%$ and that this value does not vary significantly from 100 up to $\sim 500 \mathrm{pc}$ scales in our galaxy sample.

density relative to what is seen in the extranuclear regions. This is largely opposite to what we would expect if there was an additional nonthermal component powering the $33 \mathrm{GHz}$ emission in the central regions of these galaxies, unless the excess dust heating at $24 \mu \mathrm{m}$ far exceeds any additional nonthermal emission contribution at $33 \mathrm{GHz}$. 

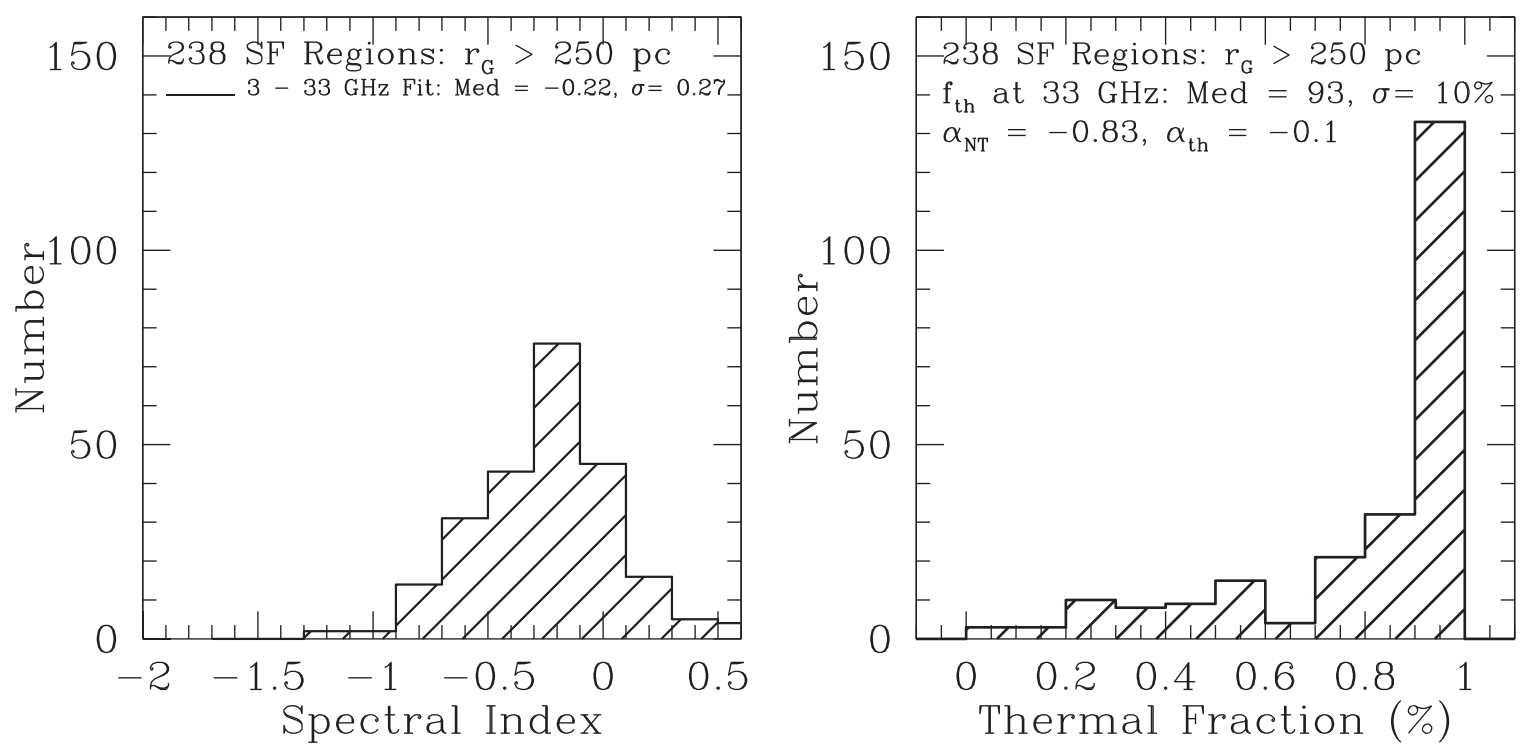

Figure 4. Spectral index and thermal fraction distributions for all $238 \mathrm{SF}$ regions with the likely supernova remnants and AME candidates removed and a requirement of $r_{\mathrm{G}} \geqslant 250 \mathrm{pc}$ (the same cut adopted in M18a). This represents the cleanest sample of extranuclear star-forming regions for which we can determine broad population statistics for extragalactic $\mathrm{H}$ II regions. Ultimately we find that the median spectral index and thermal fraction distributions at $33 \mathrm{GHz}$ are consistent with the results presented in Figures 2 and 3, confirming that supernova remnants, ultracompact $\mathrm{H}$ II regions, and AME candidates do not bias our results.

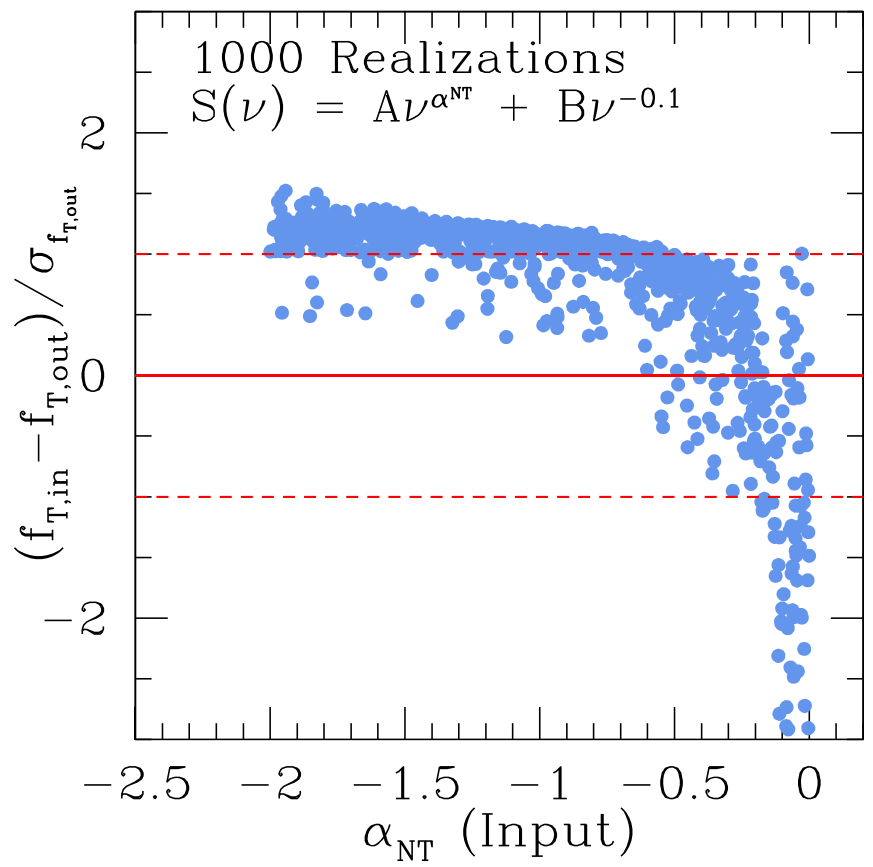

Figure 5. Results from our MCMC analysis of 1000 random realizations of 3 $-33 \mathrm{GHz}$ spectra using as a model $S(\nu)=A \nu^{\alpha^{N \mathrm{~T}}}+B \nu^{-0.1}$. For these realizations we fix $\mathrm{S} / \mathrm{N}=10$ and vary the thermal fraction at $33 \mathrm{GHz}$ by randomly assigning a nonthermal spectral index from 0 to 2 and $A(B)$ values between 0 and 1 . It is clear from this exercise that by fitting our data using an MCMC approach, our ability to recover the true value for the $33 \mathrm{GHz}$ thermal fraction peaks at $\alpha^{\mathrm{NT} \text {, in }} \sim-0.8$, which closely resembles the canonical value for the nonthermal spectral index.

Therefore, these results suggested that the larger ratio of $33 \mathrm{GHz}$ flux density to $\mathrm{H} \alpha$ line flux found in the central regions of these galaxies may primarily arise from increased extinction. We can now test this picture for 325 discrete regions (background galaxies removed) with detailed radio spectral fitting and thermal fraction estimates, which do not suffer from the effects of variable dust extinction in galaxies. In Figure 6 it is clear that the overall dispersion in the measured spectral index and thermal fraction at $33 \mathrm{GHz}$ increases significantly for regions that lie within the $250 \mathrm{pc}$ galactocentric radius cut used in M18a to distinguish extranuclear from nuclear/circumnuclear star-forming regions. In fact, limiting the analysis to sources with $r_{\mathrm{G}}<250 \mathrm{pc}$ results in a value for the median $33 \mathrm{GHz}$ thermal fraction of $\sim 71 \% \pm 3.5 \%$ with a median absolute deviation of $\sim 18 \%$ relative to $\sim 95 \% \pm 2.2 \%$ with a median absolute deviation of $\sim 11 \%$ for regions with $r_{\mathrm{G}} \geqslant$ $250 \mathrm{pc}$. Additionally, the scatter of the thermal fraction distribution increases by nearly a factor of 2 within $r_{\mathrm{G}}<250 \mathrm{pc}$. This confirms that while extinction may play a role in driving the previously seen correlations, excess nonthermal emission is indeed present in many of the circumnuclear star-forming regions observed in the SFRS.

\subsection{Model Age Fitting}

By making use of differences in the timescales associated with thermal (free-free) and synchrotron emission, we can place estimates on the age of star-forming regions by examining how these processes affect the radio spectral indices from 3 to $33 \mathrm{GHz}$. Since free-free emission is directly associated with ionizing photons that are only produced by the shortest-lived ( $\leqslant 10 \mathrm{Myr}$ ) massive stars, its presence in large quantities relative to synchrotron emission is indicative of very young star formation.

To better quantify these different timescales, we use a Starburst 99 (SB99) model of a single instantaneous burst with default inputs (solar metallicity and two-component Kroupa IMF) run for $1 \mathrm{Gyr}$ (Leitherer et al. 1999). However, in order to take into account the fact that at the median physical scales we are probing $(\sim 100 \mathrm{pc})$ a single, instantaneous starburst may not be representative, we also include SB99 models with a continuous $\mathrm{SFH}$ of $\mathrm{SFR}=1 M_{\odot} \mathrm{yr}^{-1}$ using the same metallicity and IMF input as in the instantaneous burst model (Figure 7, left panel). The details of these models and their 



Figure 6. Spectral index and $33 \mathrm{GHz}$ thermal fraction distributions plotted against galactocentric radius for all 325 sources identified in Figure 2 . While we identify regions across the full extent of galaxy disks that are heavily dominated by thermal emission, a clear trend emerges where the scatter in both quantities increases significantly as a function of decreasing galactocentric radius (orange points). In particular, no region with a measured $f_{\mathrm{T}}<80 \%$ is found in any SFRS galaxy beyond a radius of $7 \mathrm{kpc}$. These trends are reflective of the ongoing star formation activity occurring in the centers of nearby normal galaxies and reinforce our ability to successfully capture the SFH of individual $\mathrm{H}$ II regions using the $3-33 \mathrm{GHz}$ radio spectral slopes.


Figure 7. Left panel: evolution of the 3-33 GHz spectral slope in SB99 models of both an instantaneous burst and continuous SFH, using standard Kroupa IMF and solar metallicities. We perform a $\chi^{2}$-minimization of these models to the observed 3-33 GHz spectral index of each region. Right panel: distribution of model ages for both types of SFH (blue and green) and the best-fitting model in each case (gray). It is clear that there exist two populations of regions: those younger than $t \sim 10 \mathrm{Myr}$, which are best modeled by an instantaneous burst, and those older than $t \sim 10 \mathrm{Myr}$, which are best modeled by a continuous SFH. 
application to extranuclear star-forming regions identified in LIRGs are presented in Linden et al. (2019).

For both models we then perform a $\chi^{2}$ minimization to the observed spectral indices for each star-forming region in the sample. We stress that without including CR propagation, which will affect the relative ratio of free-free and nonthermal emission on these scales, this exercise is simply meant to understand how the observed distribution of spectral indices can be represented as a distribution of ages. In the left panel of Figure 7 it is clear that our models are insensitive to $\mathrm{H} \mathrm{II}$ regions with ages $\tau_{\mathrm{H} \mathrm{II}}<3 \mathrm{Myr}$ and $\tau_{\mathrm{H} \mathrm{II}}>40 \mathrm{Myr}$. However, in the intermediate age range we find that a typical uncertainty of $4 \%-6 \%$ on the observed spectral index corresponds to a $0.1-0.2$ dex uncertainty in age. Therefore, while the age of any individual region may be uncertain, the increase in the number of young $\left(3<\tau_{\mathrm{H} \text { II }}<10 \mathrm{Myr}\right)$ relative to old $\left(\tau_{\mathrm{H} \text { II }} \sim 20-30\right.$ Myr) star-forming regions is robust.

The right panel of Figure 7 shows the distribution of fitted ages for the instantaneous burst model in blue and the continuous model in green. Overall, we find that the majority $(\sim 70 \%)$ of our regions are best modeled by a continuous burst, with a median age of $\sim 10 \mathrm{Myr}$ (gray distribution). Further, we find that when all regions are modeled using a continuous $\mathrm{SFH}$, an age gradient emerges in Figure 6. At small $r_{\mathrm{G}}$, the regions with low thermal fractions, which drive the observed scatter, are preferentially older $\left(\tau_{\mathrm{HII}} \sim 20-30 \mathrm{Myr}\right)$. This further supports the notion that the central star-forming regions are, on average, older, and that the associated CR population in the inner disk of these galaxies is being continuously replenished by ongoing star formation.

\subsection{Likely Background Galaxies}

For the 10 likely background sources (BG) identified (i.e., sources with no obvious $8 \mu \mathrm{m}$ counterpart), which have an $\mathrm{S} / \mathrm{N} \geqslant 3$ in at least two radio bands, the median $3-33 \mathrm{GHz}$ spectral index is $-0.65 \pm 0.04$ with a median absolute deviation of 0.3 . This value is significantly steeper than the average value measured for the SF regions and indicates that these sources are primarily dominated by synchrotron emission. This result further suggests that our visual classification scheme involving both radio and near-IR imaging appears to be an effective discriminator for various types of radio sources in surveys of nearby galaxies. Finally, a cross-reference with NED suggests that none of these sources have been previously identified in the literature.

\subsection{Supernovae and Supernova Remnants}

In order to identify possible supernova remnants, we crosscorrelated our sample of 377 regions against the Open Supernova Catalog (OSC; Guillochon et al. 2017). In total we identify six sources as being spatially coincident (within $2^{\prime \prime}$ ) to an identified radio source with an $\mathrm{S} / \mathrm{N} \geqslant 3$ in at least two radio bands. The $3-33 \mathrm{GHz}$ spectral slopes measured are uniformly distributed from -1 to 0.5 . This scatter is likely driven by $\mathrm{SNe} / \mathrm{R}$ at various stages of their evolution, and therefore a large range in the degree of energy loss of the CRs as they propagate through the ISM. For one region identified in NGC 7331, the measured $15 \mathrm{GHz}$ flux density is larger by over an order of magnitude as a result of a supernova, SN 2014C, which was discovered in January of 2014, between the time our
3 and $15 \mathrm{GHz}$ observations of this source were taken (Shivvers et al. 2019).

\subsection{Anomalous Microwave Emission}

AME is a known dust-correlated component of Galactic emission that has been detected by cosmic microwave background $(\mathrm{CMB})$ experiments and other radio/microwave instruments at frequencies $10-60 \mathrm{GHz}$ since the mid-1990s (see Dickinson et al. 2018 and articles within for recent reviews). AME is found to be spatially correlated with FIR thermal dust emission but cannot be explained by synchrotron or free-free emission mechanisms and is far in excess of the emission contributed by thermal dust with the power-law opacity consistent with observations at submillimeter wavelengths. The most natural explanation for AME is rotational (electric dipole) emission from ultrasmall dust grains (i.e., "spinning dust"; Erickson 1957; Draine \& Lazarian 1998). The emission forms part of the diffuse Galactic foregrounds that contaminate $\mathrm{CMB}$ data, which operate in the frequency range 30-300 GHz, and hence knowledge of the spatial structure and spectral shape can inform CMB component separation. However, spinning dust emission depends critically on the dust grain size distribution, the type of dust, and the environmental conditions (density, temperature, interstellar radiation field, etc.). Thus, precise measurements of AME can also provide a new window into the ISM, complementing other multiwavelength tracers.

A number of searches for extragalactic AME have been undertaken with WMAP and Planck data (e.g., the Magellanic Clouds and NGC 4945; Bot et al. 2010; Peel et al. 2011), all of which were inconclusive. Most recently, we have identified two additional detections of AME in the SFRS sample as having anomalously high $33 \mathrm{GHz}-24 \mu \mathrm{m}$ flux ratios (NGC 6946 E4 and NGC 4725 B; Murphy et al. 2010, 2018b). NGC 4725 B in particular appears consistent with a highly embedded $\left(A_{V}>\right.$ 5 mag) nascent star-forming region, in which young ( $3 \mathrm{Myr}$ ) massive stars are still enshrouded by their natal cocoons of gas and dust, lacking enough supernovae to produce synchrotron emission.

While it is possible that NGC $4725 \mathrm{~B}$ and NGC $6946 \mathrm{E} 4$ represent the most favorable conditions for AME detection, there are likely remaining regions in the SFRS that still harbor $\mathrm{AME}$ at a lower level relative to the other emission components (Hensley et al. 2015). Isolating the factors that govern the level of $\mathrm{AME}$ in these regions will lend insight into the physical mechanisms powering this emission, as well as the nature of its carriers. In particular, we have observed that a common feature among both detections thus far is a shallow or even rising spectra from 3 to $33 \mathrm{GHz}$, as the contribution from AME increases and eventually dominates beyond $\sim 20 \mathrm{GHz}$. These regions can be identified as having elevated $33 \mathrm{GHz}$ emission relative to the expected extrapolation from lower-frequency radio data using a standard two-component power law.

By measuring the $3-33 \mathrm{GHz}$ and $15-33 \mathrm{GHz}$ spectral indices, we made an initial selection of 58 extragalactic AME candidates as regions that have an $8 \mu \mathrm{m}$ counterpart, an $\mathrm{S} / \mathrm{N} \geqslant 3$ in at least two radio bands, a deprojected galactocentric radius of $r_{\mathrm{G}}>250 \mathrm{pc}$, and a measured spectral slope that is $\geqslant 3 \sigma$ above the canonical $\alpha^{\mathrm{T}}=-0.1$ value expected for free-free emission. This represents a conservative upper limit to identify regions by assuming that the nonthermal synchrotron emission is negligible at $33 \mathrm{GHz}$, and thus does not 


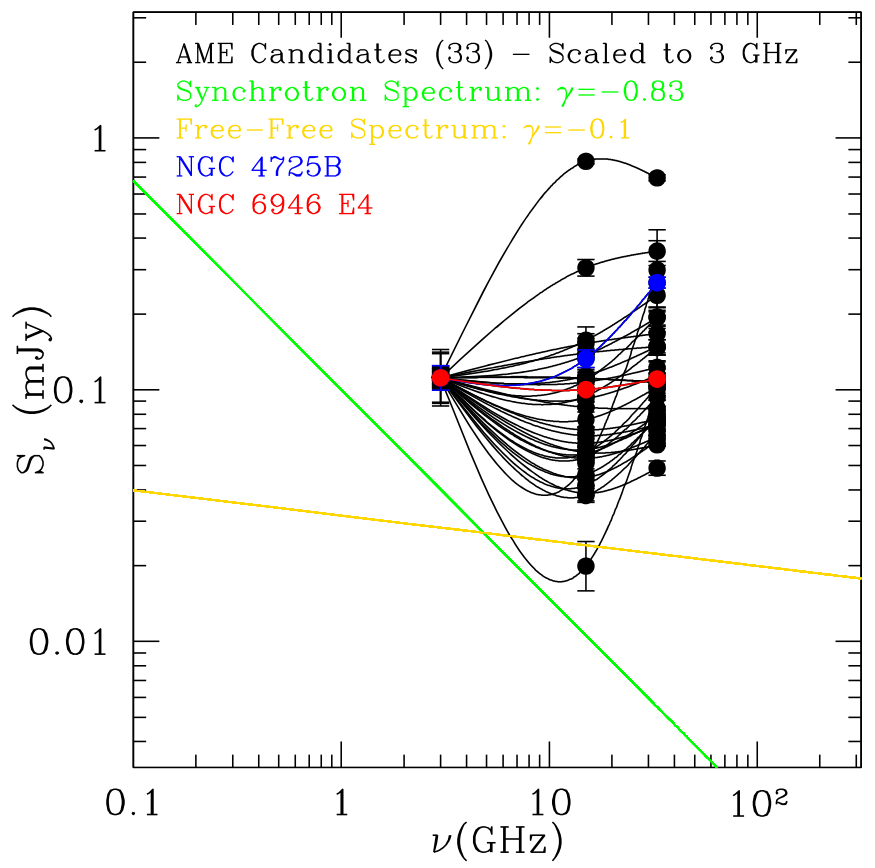

Figure 8. Radio spectra of all 33 AME candidates identified in the SFRS as having $3-33 \mathrm{GHz}$ or $15-33 \mathrm{GHz}$ spectral indices $3 \sigma$ greater than $\alpha^{\mathrm{T}}=-0.1$. To demonstrate the diversity of our AME candidate spectra, we normalize all flux density measurements to the brightest region detected at $3 \mathrm{GHz}$. We expect that, similar to NGC $6946 \mathrm{E} 4$ (red), the regions with decreasing $3-15 \mathrm{GHz}$ spectral slopes contain a nonnegligible amount of synchrotron emission, whereas the regions with very shallow or even rising spectra over this same frequency range (e.g., similar to NGC $4725 \mathrm{~B}$; blue) will be dominated by freefree emission.

contribute to the measured slope from 15 to $33 \mathrm{GHz}$. This assumption is well supported here for our full spectral analysis of over 300 star-forming regions identified in the SFRS, which show that the median thermal fraction measured at $33 \mathrm{GHz}$ on a few $\sim 100 \mathrm{pc}$ scales is $\sim 91 \%$. We then visually inspected all 58 AME candidates, only retaining 33 regions where the emission was found to be compact, similar to the previously identified extragalactic AME sources. This is done to ensure that any differences in surface brightness sensitivity between our observations would not result in diffuse emission with an artificially flattened spectrum, which is unassociated with an individual source. Our final requirement removes four sources (NGC 4254 Enuc. 1 B, NGC 3521 Enuc. 1, NGC 4736N, and NGC $7331 \mathrm{D}$ ) that have very steeply rising 3-15 GHz spectral slopes but are undetected at $33 \mathrm{GHz}$ and therefore are not confidently identified as AME candidates.

In Figure 8 we plot the $3-33 \mathrm{GHz}$ spectrum of the $33 \mathrm{AME}$ candidate regions normalized to the highest measured $3 \mathrm{GHz}$ flux density. Viewed in this way, we see that many of our AME candidates, similar to NGC $4725 \mathrm{~B}$, have shallow or slightly negative slopes from 3 to $15 \mathrm{GHz}$ and a much steeper positive slope from 15 to $33 \mathrm{GHz}$. However, there are some regions that look more similar to NGC 6946 Enuc 4., which have steeper $3-15 \mathrm{GHz}$ slopes and a less significant increase from 15 to $33 \mathrm{GHz}$. Importantly, these spectra cannot be explained by a simple combination of synchrotron (green) and free-free (yellow) emission components, suggesting that either an additional emission component peaking at $\gtrsim 15 \mathrm{GHz}$ is required (e.g., AME), it is a (very) high-frequency GHz-peaked background galaxy, or the source is variable. A final possibility is that the free-free emission is optically thick at $33 \mathrm{GHz}$. However, such ultracompact $\mathrm{H}$ II regions have much higher radio luminosities and SFRs $(\sim 40-60 \mathrm{mJy})$ than the regions identified here (e.g., Meier et al. 2002).

\section{Conclusions}

We have presented 3,15 , and $33 \mathrm{GHz}$ imaging toward galaxy nuclei and extranuclear star-forming regions in the SFRS and have identified 335 regions (286 SF, $10 \mathrm{BG}, 6 \mathrm{SNe} / \mathrm{R}$, and 33 AME) with $\mathrm{S} / \mathrm{N} \geqslant 3$ in at least two radio bands. Through detailed measurements of their radio spectra we have confirmed the following:

1. The average local background contribution to the measured 3,15 , and $33 \mathrm{GHz}$ flux densities on $\sim 100 \mathrm{pc}$ scales is $\sim 4 \%-6 \%$. This is significantly smaller than the $15 \%-40 \%$ found for the sample of regions studied at $25^{\prime \prime}$ ( $\sim 1 \mathrm{kpc}$ ) scales with the GBT (Murphy et al. 2011).

2. On $\sim 100 \mathrm{pc}$ scales, the median thermal fraction at $33 \mathrm{GHz}$ of all regions removing background galaxies is $92 \% \pm 0.8 \%$ with a median absolute deviation of $11 \%$. Limiting our analysis to extranuclear $\left(r_{\mathrm{G}}>250 \mathrm{pc}\right) \mathrm{SF}$ regions, we measure a median thermal fraction of $93 \% \pm 0.8 \%$ with a median absolute deviation of $10 \%$. Further, we find that on $7^{\prime \prime}$ scales the median thermal fraction is $94 \% \pm 0.8 \%$, and thus the thermal fraction remains $\geqslant 90 \%$ up to $\sim 500 \mathrm{pc}$ scales.

3. We have confirmed through MCMC analysis that we do not introduce systematic biases when interpreting the results of the $\chi^{2}$-minimization of a two-component power-law model to fit the observed radio spectrum from 3 to $33 \mathrm{GHz}$, and that this model can adequately separate the thermal free-free and nonthermal synchrotron emission components over a realistic range of input values.

4. We find a systematic increase in the scatter of the measured spectral indices and thermal fractions as the deprojected galactocentric radius approaches the nucleus. This trend is reflective of the ongoing star formation activity occurring in centers of these galaxies and results in a larger contribution of diffuse nonthermal emission.

5 . We have identified a sample of 33 sources whose rising 15-33 GHz emission may be due to AME. Follow-up observations at high $(\geqslant 40 \mathrm{GHz})$ frequencies will be necessary to confirm these sources as discrete regions of extragalactic AME.

S.T.L. was supported by the NRAO Grote Reber Dissertation Fellowship. The National Radio Astronomy Observatory is a facility of the National Science Foundation operated under cooperative agreement by Associated Universities, Inc. This research has made use of the NASA/IPAC Extragalactic Database (NED), which is operated by the Jet Propulsion Laboratory, California Institute of Technology, under contract with the National Aeronautics and Space Administration.

\section{Appendix}

In the following appendix, we present the remaining $3-33 \mathrm{GHz}$ three-panel images (Figures A1-A15), the Ancillary UV and $\mathrm{H} \alpha$ photometry at 7" angular resolution (Table A1), and the local background values measured for the apertures adopted in Table 4 (Table A2). 


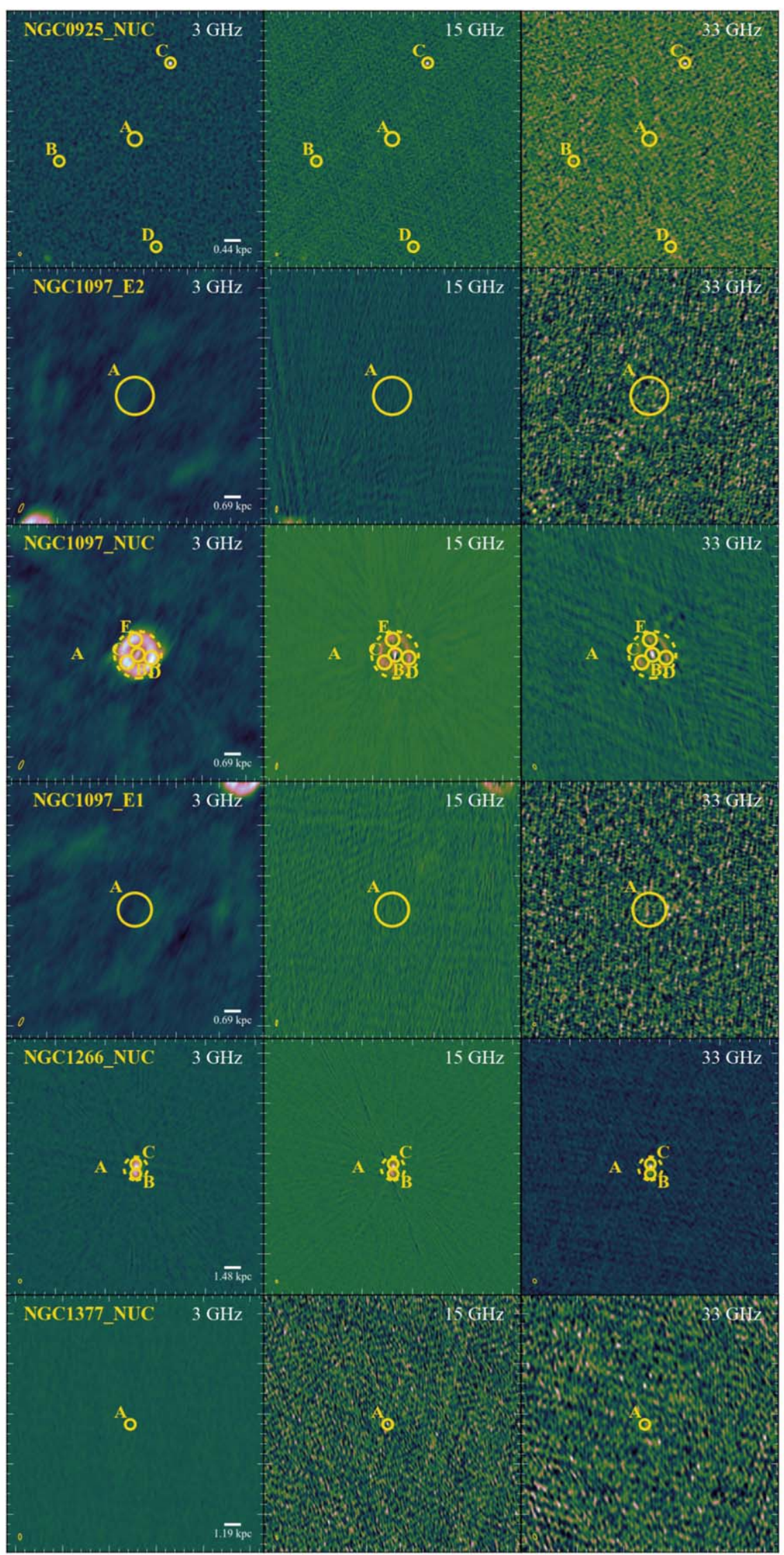

Figure A1. See description in Figure 1. 


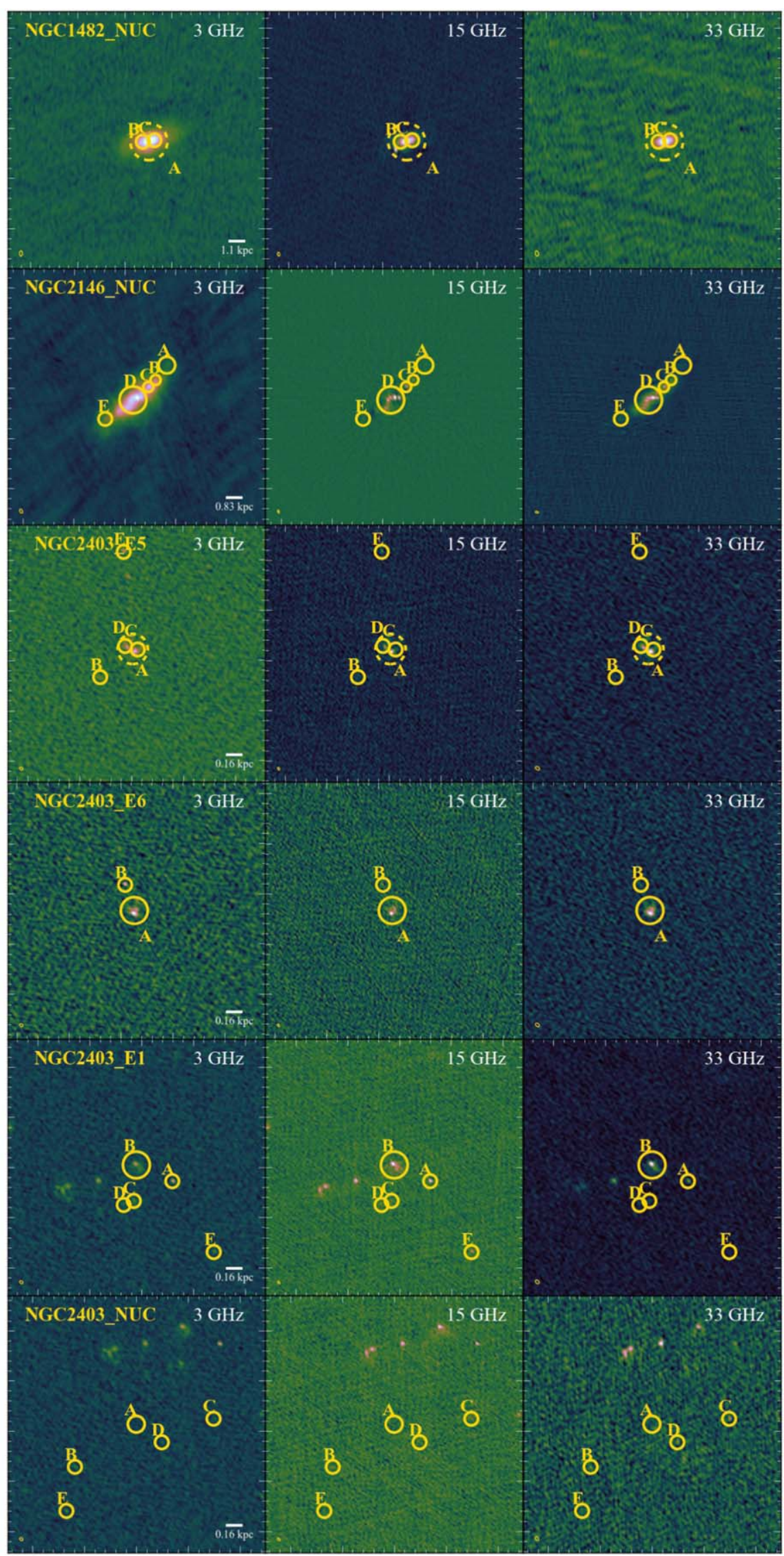

Figure A2. See description in Figure 1. 


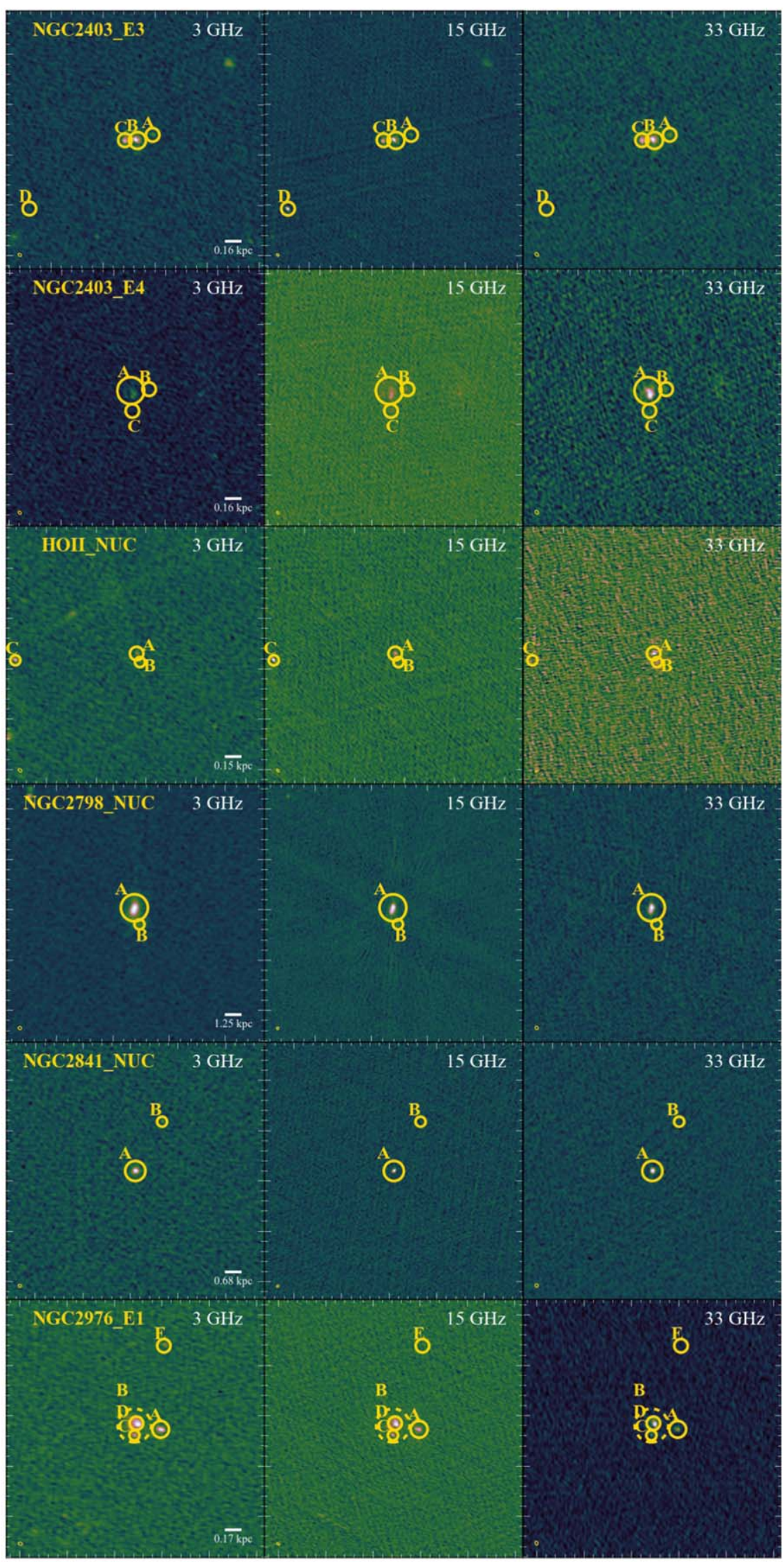

Figure A3. See description in Figure 1. 




Figure A4. See description in Figure 1. 


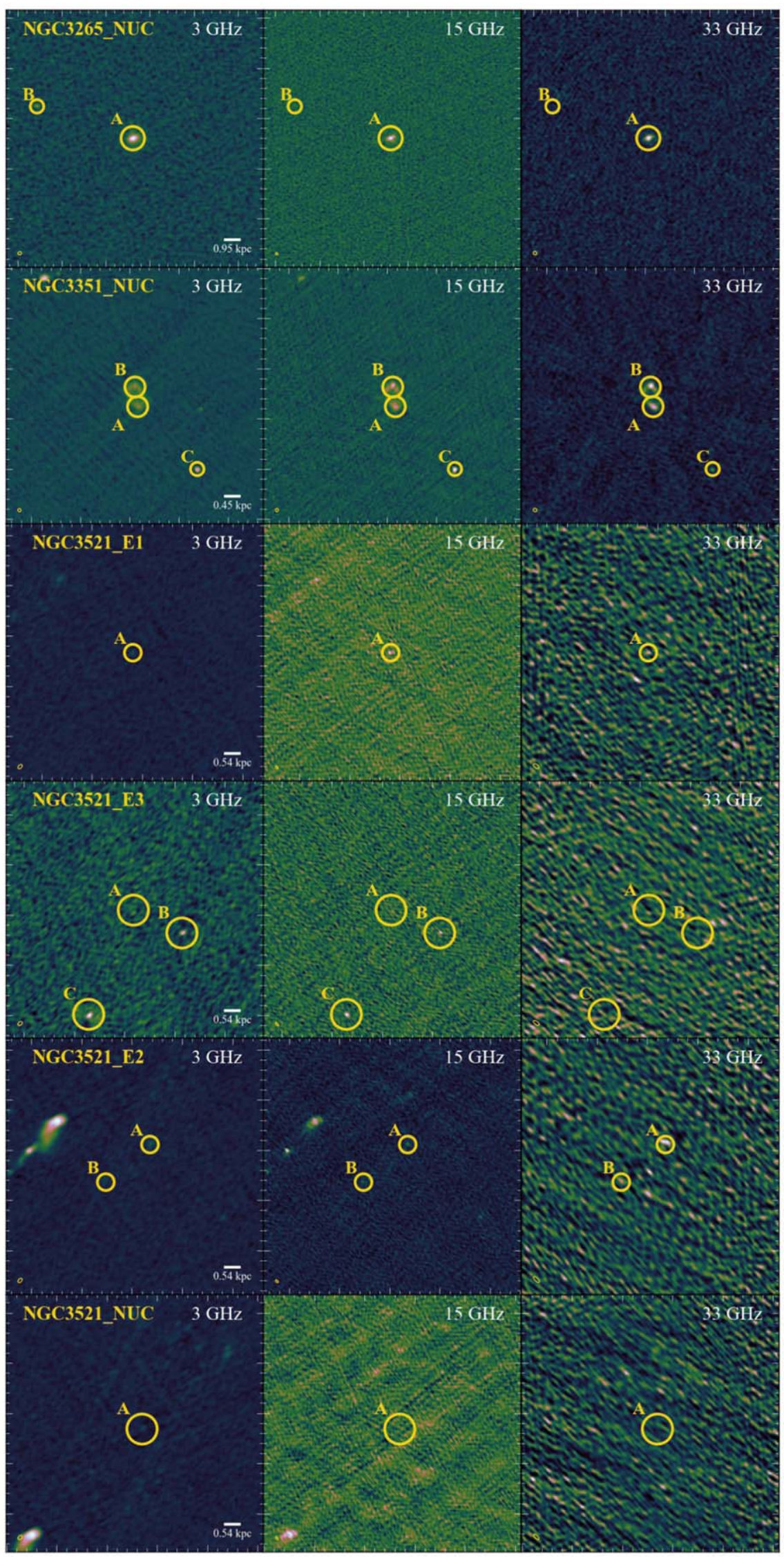

Figure A5. See description in Figure 1. 

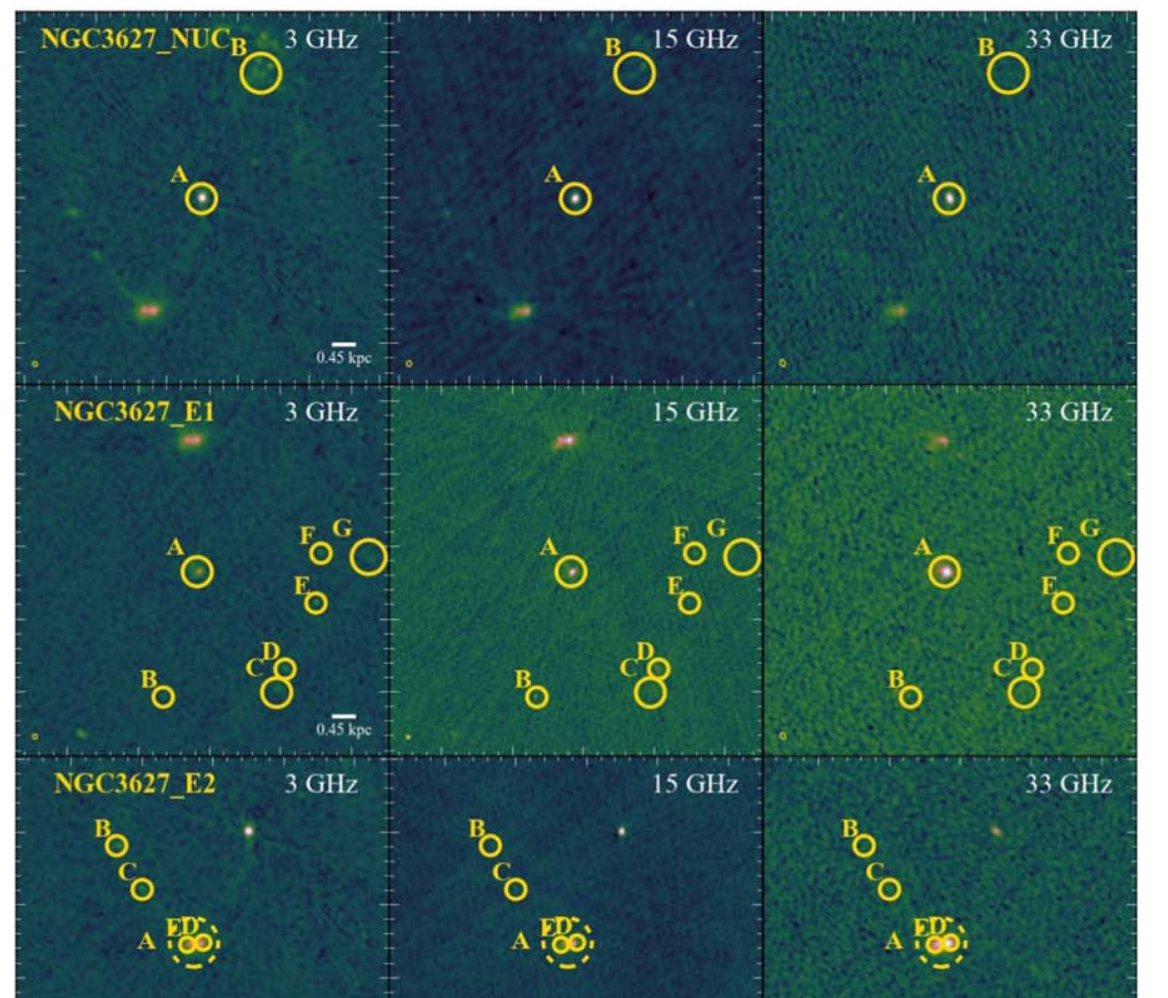


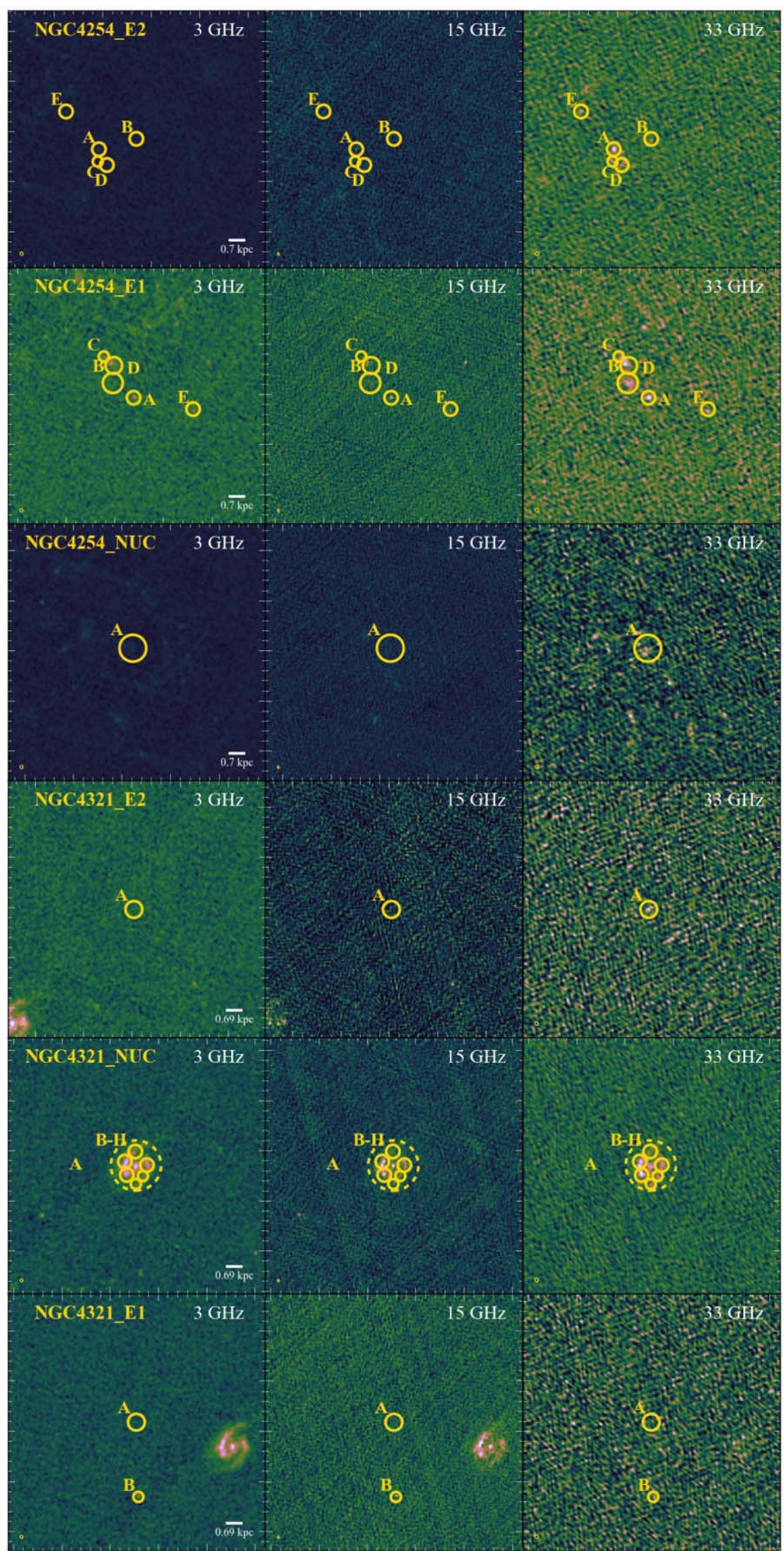

Figure A7. See description in Figure 1. 


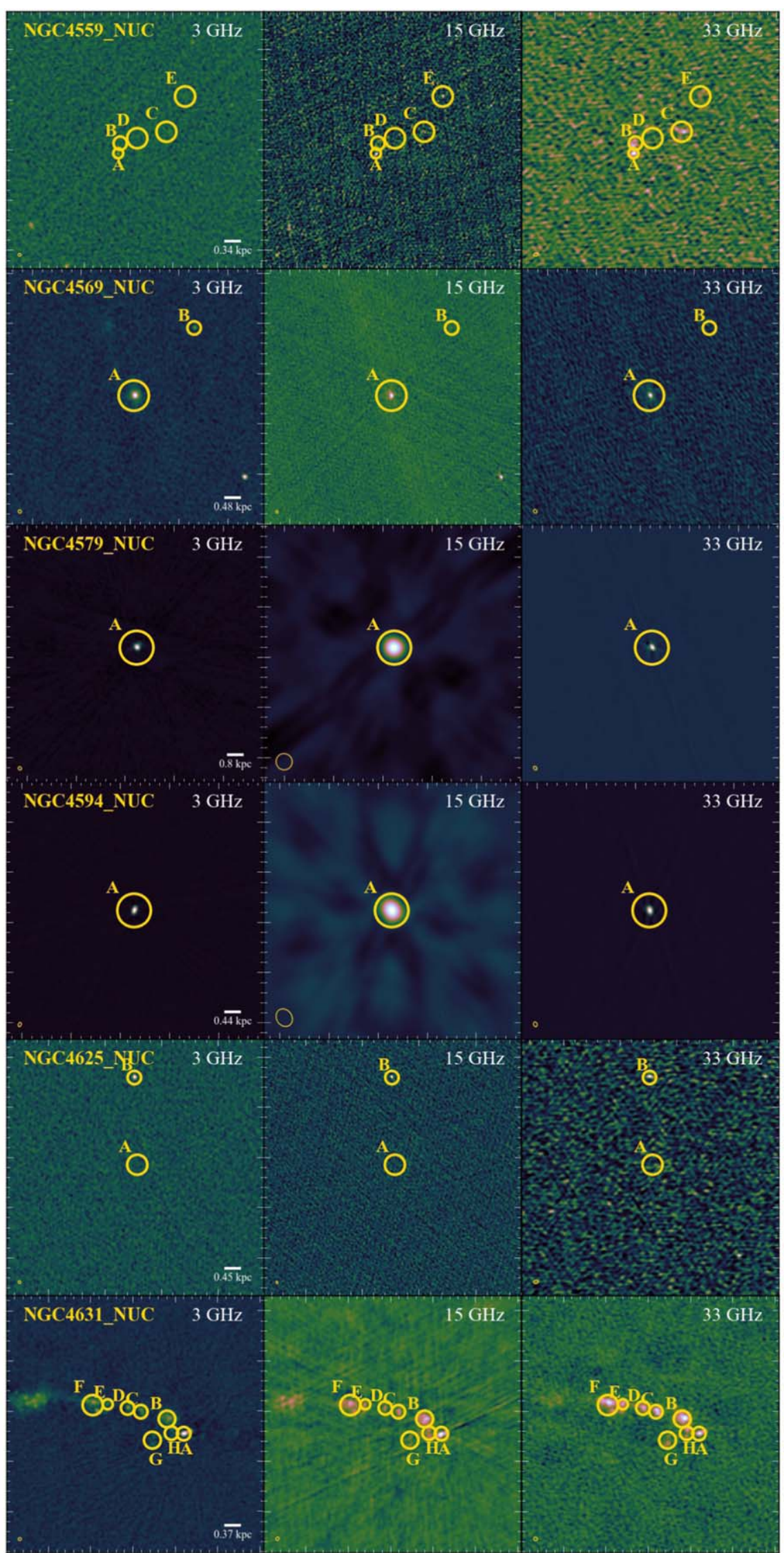

Figure A8. See description in Figure 1. 


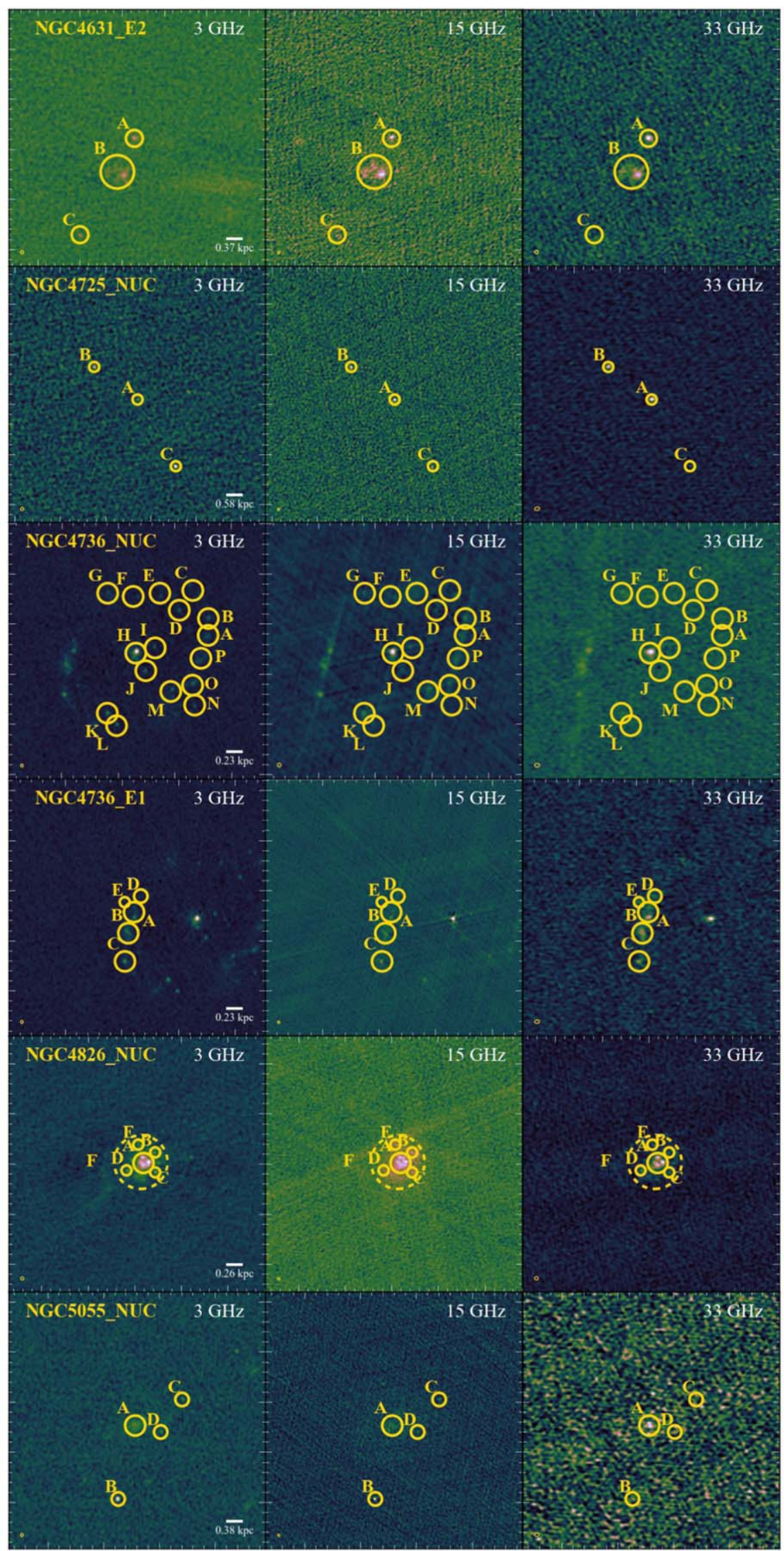

Figure A9. See description in Figure 1. 


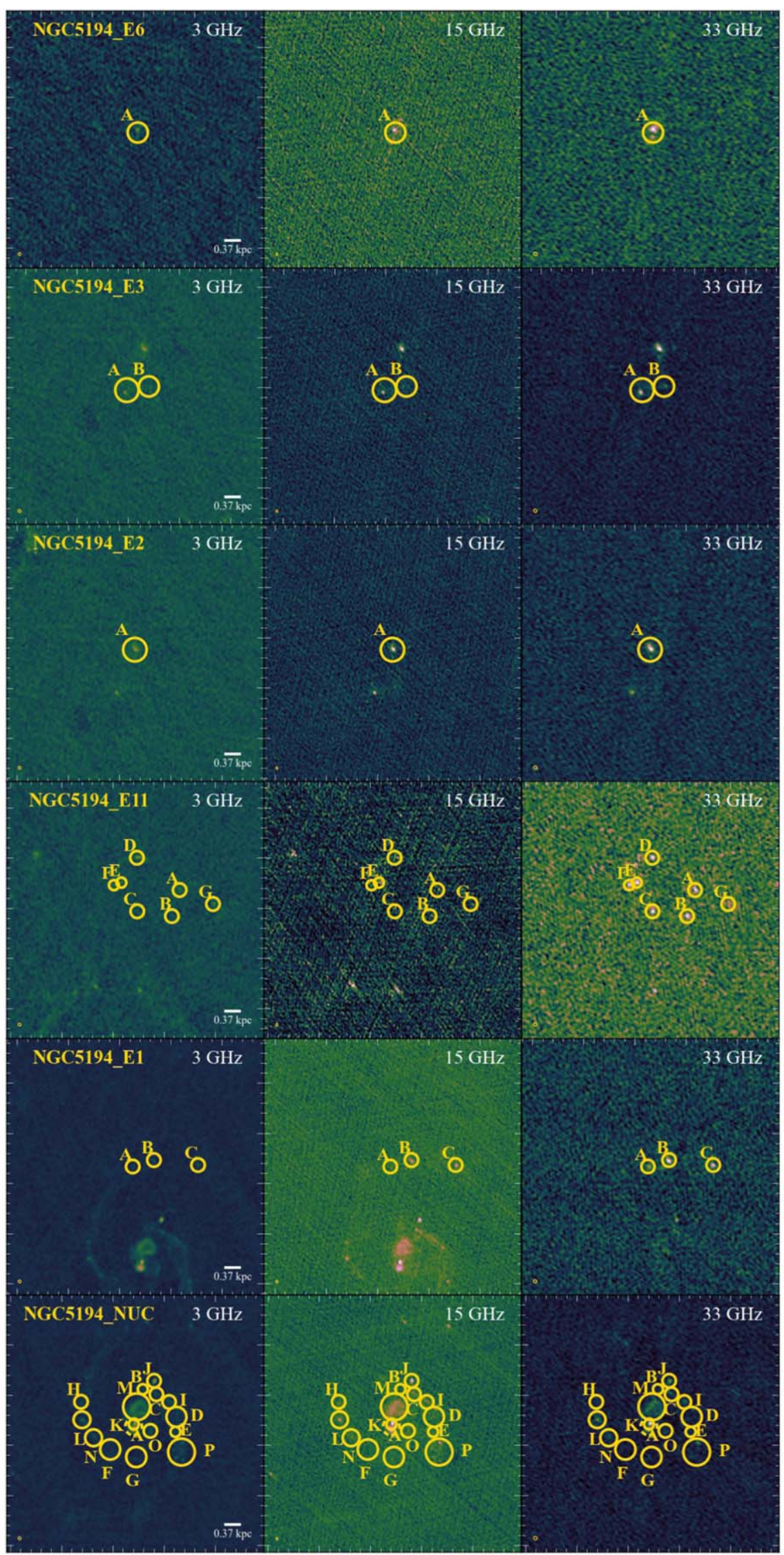

Figure A10. See description in Figure 1. 


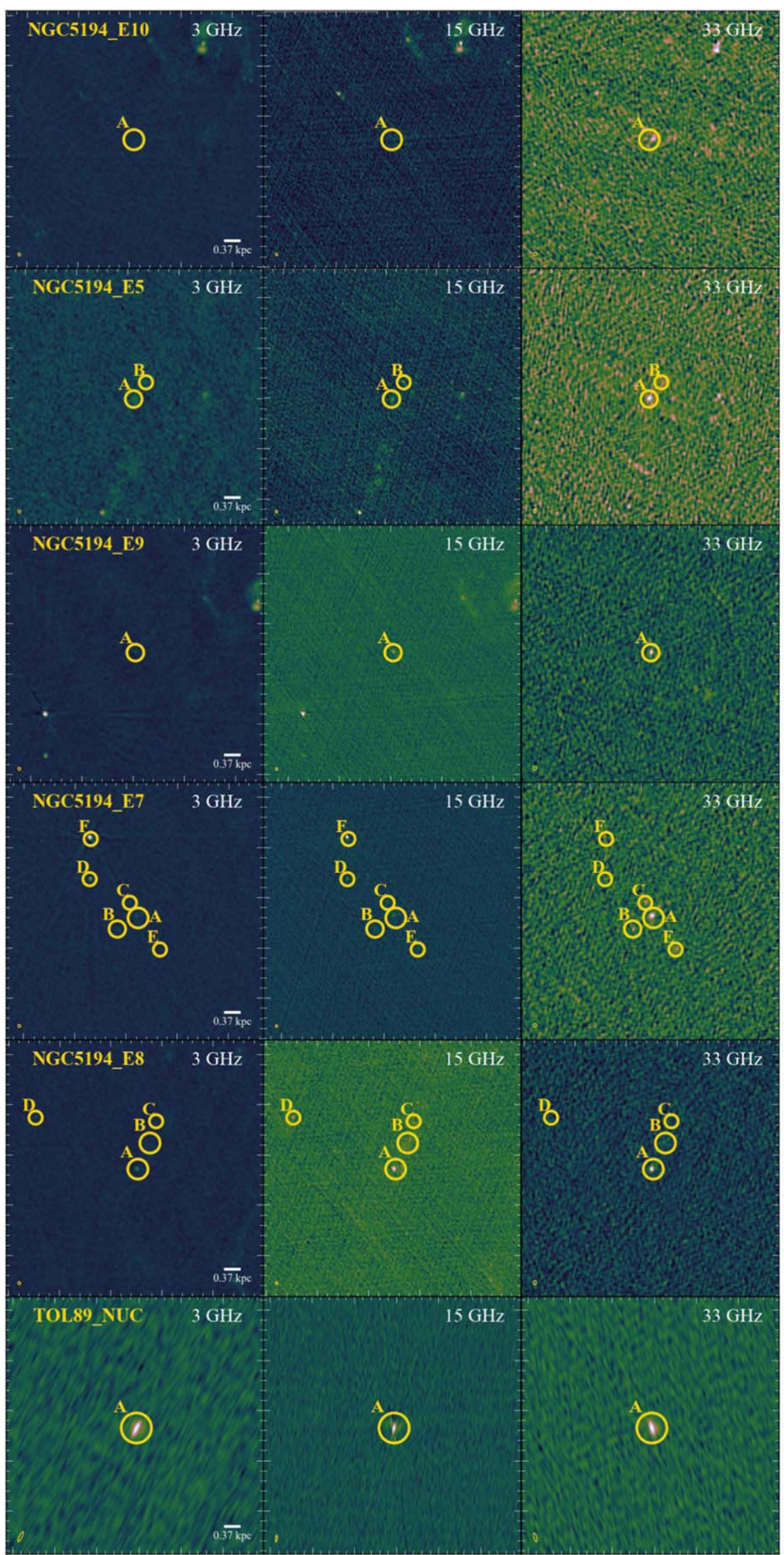

Figure A11. See description in Figure 1. 


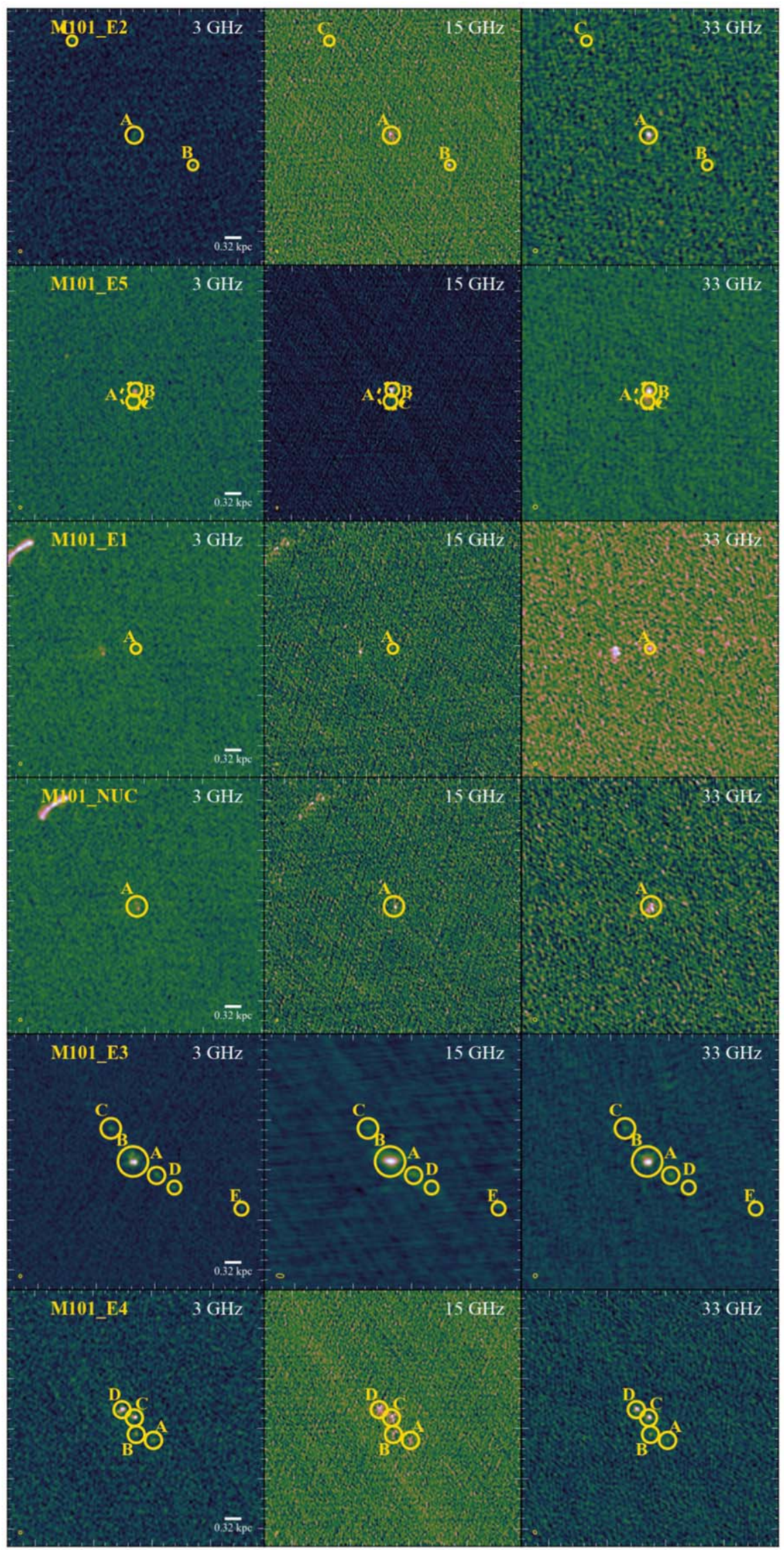

Figure A12. See description in Figure 1. 


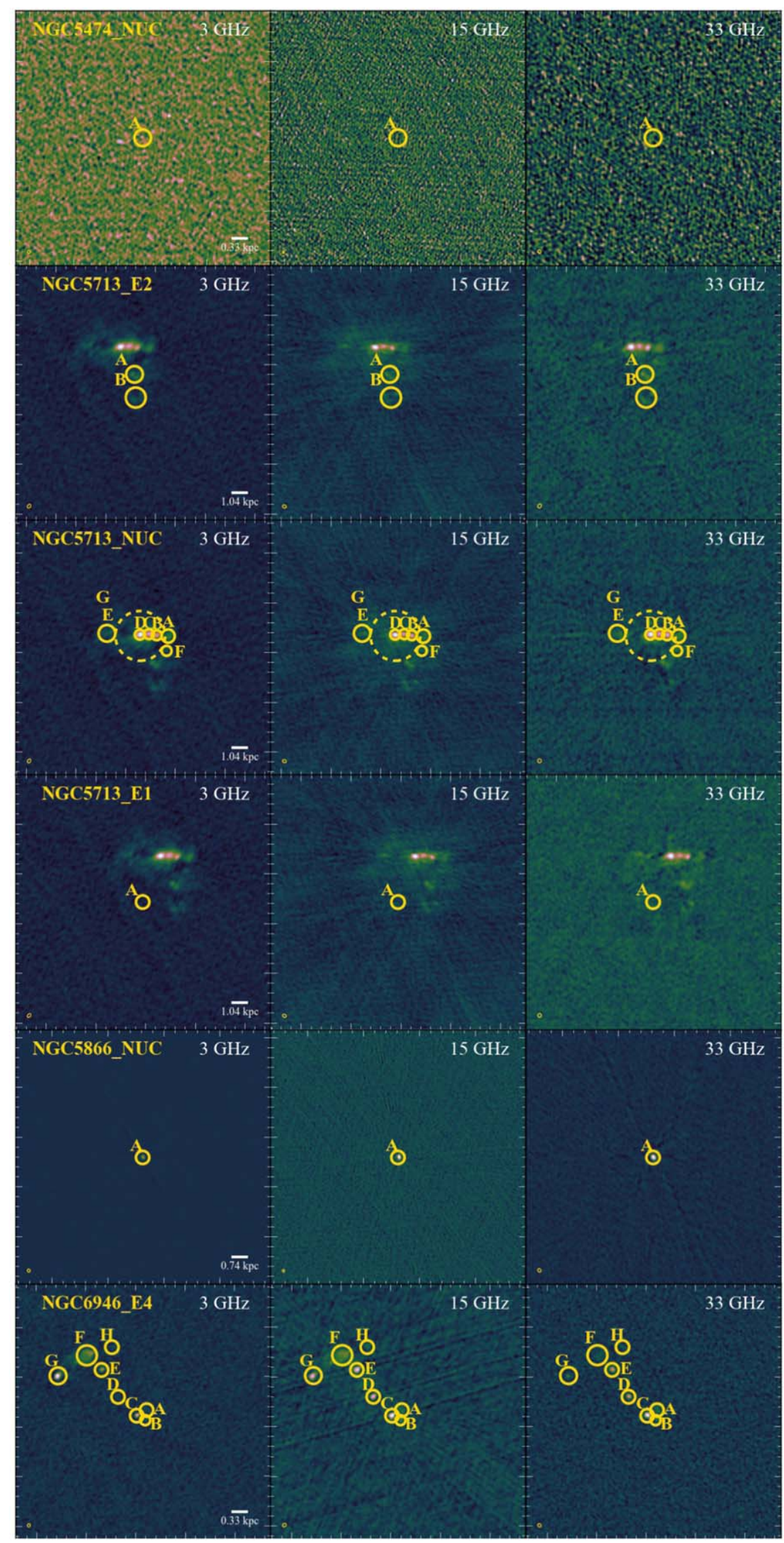

Figure A13. See description in Figure 1. 


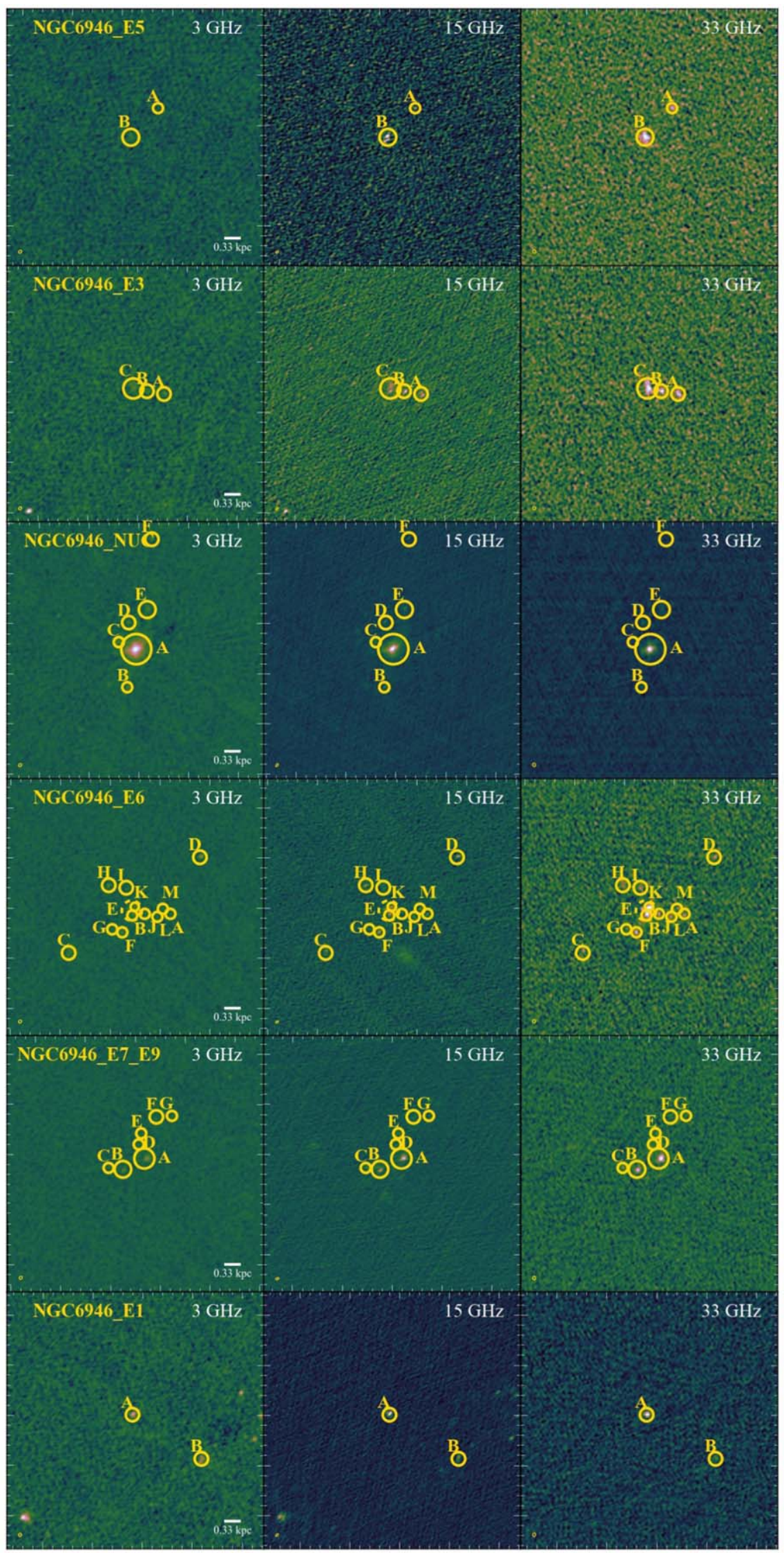

Figure A14. See description in Figure 1. 


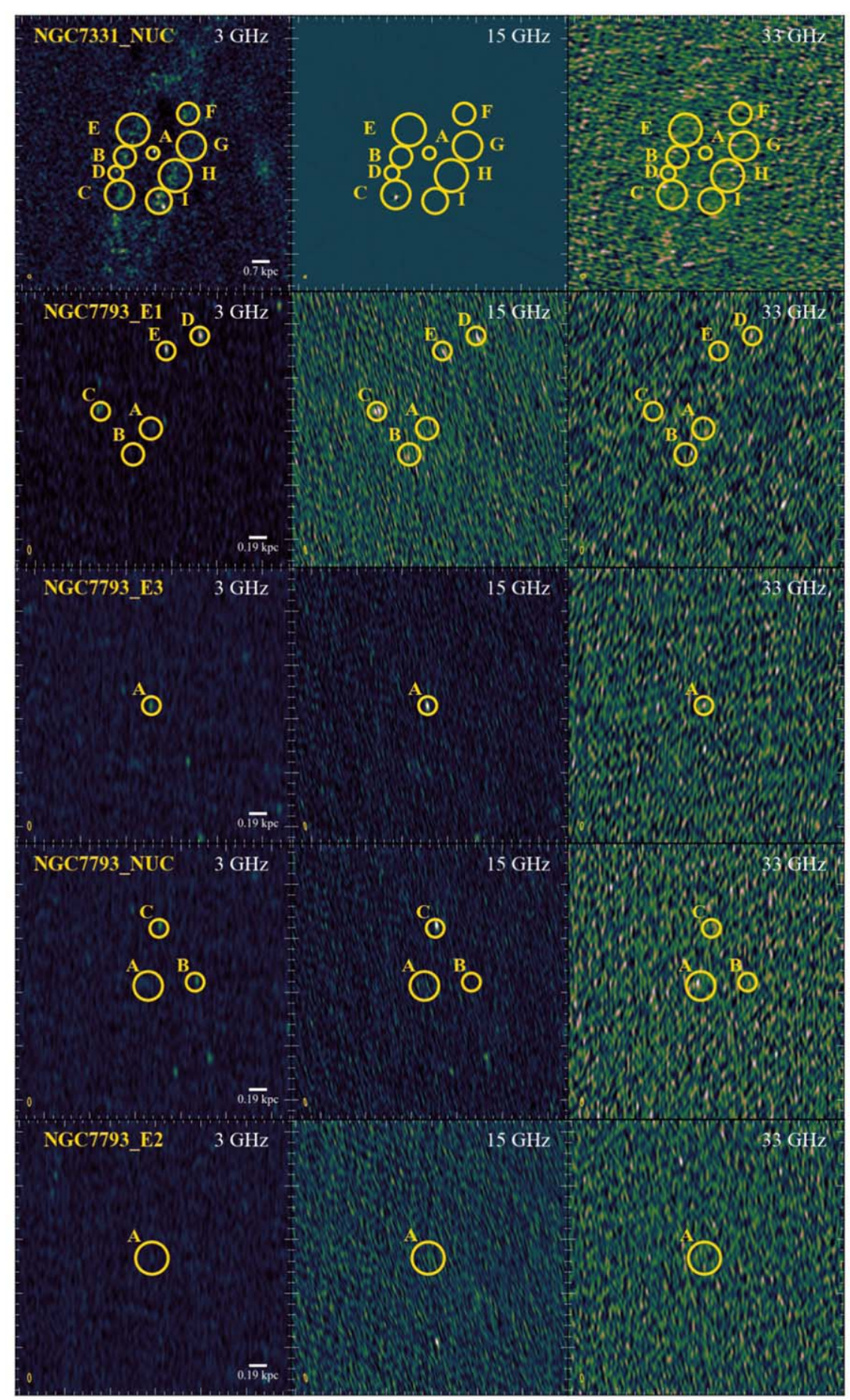

Figure A15. See description in Figure 1.

Table A1

Ancillary Source Photometry at 7" Angular Resolution

\begin{tabular}{|c|c|c|c|c|}
\hline Source ID & $\begin{array}{c}f_{\nu}(1528 \AA) \\
(\mu \mathrm{Jy})\end{array}$ & $\begin{array}{c}f_{\nu}(2271 \AA) \\
(\mu \mathrm{Jy})\end{array}$ & $\begin{array}{c}f_{\mathrm{H} \alpha} / 10^{-13} \\
\left(\mathrm{erg} \mathrm{s}^{-1} \mathrm{~cm}^{-2}\right)\end{array}$ & $\begin{array}{c}f_{\nu}(24 \mu \mathrm{m}) \\
(\mathrm{mJy})\end{array}$ \\
\hline \multicolumn{5}{|c|}{ Star-forming Regions } \\
\hline NGC 0337 a & $76.38 \pm 11.46$ & $163.59 \pm 24.54$ & $1.47 \pm 0.29$ & $45.73 \pm 2.29$ \\
\hline NGC 0337 b & $104.70 \pm 15.71$ & $185.04 \pm 27.76$ & $1.83 \pm 0.37$ & $81.65 \pm 4.08$ \\
\hline NGC 0337 c & $36.93 \pm 5.54$ & $70.52 \pm 10.58$ & $0.57 \pm 0.11$ & $8.40 \pm 0.42$ \\
\hline NGC $0337 \mathrm{~d}$ & $47.19 \pm 7.08$ & $87.63 \pm 13.15$ & $1.18 \pm 0.24$ & $22.25 \pm 1.11$ \\
\hline NGC 0628 Enuc. 4 & $67.64 \pm 10.15$ & $81.23 \pm 12.19$ & $0.53 \pm 0.11$ & $9.87 \pm 0.50$ \\
\hline
\end{tabular}


Table A1

(Continued)

\begin{tabular}{|c|c|c|c|c|}
\hline Source ID & $\begin{array}{c}f_{\nu}(1528 \AA ̊ \AA) \\
(\mu \mathrm{Jy})\end{array}$ & $\begin{array}{c}f_{\nu}(2271 \AA) \\
(\mu \mathrm{Jy})\end{array}$ & $\begin{array}{c}f_{\mathrm{H} \alpha} / 10^{-13} \\
\left(\mathrm{erg} \mathrm{s}^{-1} \mathrm{~cm}^{-2}\right)\end{array}$ & $\begin{array}{c}f_{\nu}(24 \mu \mathrm{m}) \\
(\mathrm{mJy})\end{array}$ \\
\hline NGC 0628 Enuc. 2 & $70.55 \pm 10.58$ & $95.46 \pm 14.32$ & $0.40 \pm 0.08$ & $40.37 \pm 2.02$ \\
\hline NGC 0628 Enuc. 3 & $233.35 \pm 35.00$ & $282.27 \pm 42.34$ & $0.78 \pm 0.16$ & $28.94 \pm 1.45$ \\
\hline NGC 0628 & $16.96 \pm 2.54$ & $41.26 \pm 6.19$ & $0.06 \pm 0.01$ & $2.41 \pm 0.14$ \\
\hline NGC 0628 Enuc. 1 & $94.88 \pm 14.23$ & $134.80 \pm 20.22$ & $0.73 \pm 0.15$ & $75.27 \pm 3.76$ \\
\hline NGC 0855 & $146.72 \pm 22.01$ & $226.75 \pm 34.01$ & $\ldots$ & $18.70 \pm 0.94$ \\
\hline NGC 0925 & $182.62 \pm 27.39$ & $232.19 \pm 34.83$ & $0.34 \pm 0.07$ & $2.79 \pm 0.15$ \\
\hline NGC 1097 Enuc. 2 & $21.07 \pm 3.16$ & $30.92 \pm 4.64$ & $\ldots$ & $3.48 \pm 0.18$ \\
\hline NGC 1097 & $97.34 \pm 14.60$ & $242.71 \pm 36.41$ & $\ldots$ & $174.28 \pm 8.71$ \\
\hline NGC 1097 Enuc. 1 a & $20.37 \pm 3.06$ & $32.96 \pm 4.95$ & $\cdots$ & $10.91 \pm 0.55$ \\
\hline NGC 1097 Enuc. 1 b & $27.26 \pm 4.09$ & $42.24 \pm 6.34$ & $\ldots$ & $8.85 \pm 0.45$ \\
\hline NGC 1097 Enuc. $1 \mathrm{c}$ & $61.48 \pm 9.22$ & $77.38 \pm 11.61$ & $\ldots$ & $7.43 \pm 0.38$ \\
\hline NGC 1266 & $2.75 \pm 0.48$ & $10.77 \pm 1.64$ & $\cdots$ & $365.58 \pm 18.28$ \\
\hline NGC 1377 & $\ldots$ & $\ldots$ & $\cdots$ & $682.96 \pm 34.15$ \\
\hline NGC 1482 & $10.33 \pm 1.56$ & $31.06 \pm 4.66$ & $\ldots$ & $1279.02 \pm 63.95$ \\
\hline NGC 2403 Enuc. 6 & $354.10 \pm 53.12$ & $402.03 \pm 60.30$ & $1.19 \pm 0.24$ & $9.02 \pm 0.45$ \\
\hline NGC 2403 Enuc. 1 a & $48.36 \pm 7.25$ & $67.64 \pm 10.15$ & $0.14 \pm 0.03$ & $3.70 \pm 0.18$ \\
\hline NGC 2403 Enuc. 1 b & $1732.39 \pm 259.86$ & $2032.07 \pm 304.81$ & $2.70 \pm 0.54$ & $84.01 \pm 4.20$ \\
\hline NGC 2403 Enuc. 2 a & $78.83 \pm 11.82$ & $101.84 \pm 15.28$ & $0.68 \pm 0.14$ & $34.77 \pm 1.74$ \\
\hline NGC 2403 & $42.11 \pm 6.32$ & $78.92 \pm 11.84$ & $0.15 \pm 0.03$ & $3.38 \pm 0.17$ \\
\hline NGC 2403 Enuc. 2 b & $846.79 \pm 127.02$ & $1047.48 \pm 157.12$ & $2.32 \pm 0.46$ & $58.97 \pm 2.95$ \\
\hline NGC 2403 Enuc. 4 & $183.11 \pm 27.47$ & $218.95 \pm 32.84$ & $0.79 \pm 0.16$ & $17.24 \pm 0.86$ \\
\hline Holmberg II & $373.62 \pm 56.04$ & $373.64 \pm 56.05$ & $1.42 \pm 0.28$ & $16.54 \pm 0.83$ \\
\hline NGC 2798 & $165.82 \pm 24.87$ & $412.77 \pm 61.92$ & $4.77 \pm 0.95$ & $1084.54 \pm 54.23$ \\
\hline NGC 2841 & $145.60 \pm 21.84$ & $213.22 \pm 31.98$ & $0.25 \pm 0.05$ & $12.63 \pm 0.63$ \\
\hline NGC 2976 Enuc. 1 a & $15.95 \pm 2.40$ & $24.52 \pm 3.68$ & $0.55 \pm 0.11$ & $27.12 \pm 1.36$ \\
\hline NGC 2976 Enuc. 1 b & $162.97 \pm 24.45$ & $216.89 \pm 32.53$ & $3.30 \pm 0.66$ & $129.05 \pm 6.45$ \\
\hline NGC 2976 & $63.57 \pm 9.54$ & $89.68 \pm 13.45$ & $0.54 \pm 0.11$ & $7.34 \pm 0.37$ \\
\hline NGC 2976 Enuc. 2 a & $130.65 \pm 19.60$ & $154.13 \pm 23.12$ & $1.91 \pm 0.38$ & $53.39 \pm 2.67$ \\
\hline NGC 2976 Enuc. 2 b & $60.44 \pm 9.07$ & $74.82 \pm 11.23$ & $1.11 \pm 0.22$ & $23.00 \pm 1.15$ \\
\hline NGC 3049 & $438.18 \pm 65.73$ & $736.56 \pm 110.48$ & $1.48 \pm 0.30$ & $180.25 \pm 9.01$ \\
\hline NGC 3077 & $\ldots$ & $\ldots$ & $3.35 \pm 0.67$ & $\ldots^{\mathrm{a}}$ \\
\hline NGC 3190 & $9.88 \pm 1.49$ & $57.84 \pm 8.68$ & $0.16 \pm 0.03$ & $30.72 \pm 1.54$ \\
\hline NGC 3184 & $121.52 \pm 18.23$ & $243.12 \pm 36.48$ & $0.25 \pm 0.05$ & $55.49 \pm 2.77$ \\
\hline NGC 3198 & $9.78 \pm 1.47$ & $29.67 \pm 4.45$ & $0.08 \pm 0.02$ & $176.88 \pm 8.84$ \\
\hline IC 2574 a & $622.44 \pm 93.37$ & $551.40 \pm 82.71$ & $1.72 \pm 0.34$ & $4.62 \pm 0.23$ \\
\hline IC $2574 \mathrm{~b}$ & $114.96 \pm 17.24$ & $141.24 \pm 21.19$ & $1.70 \pm 0.34$ & $19.95 \pm 1.00$ \\
\hline NGC 3265 & $164.32 \pm 24.65$ & $297.20 \pm 44.58$ & $\ldots$ & $141.65 \pm 7.08$ \\
\hline NGC 3351 a & $286.98 \pm 43.05$ & $839.02 \pm 125.85$ & $2.33 \pm 0.47$ & $307.30 \pm 15.36$ \\
\hline NGC $3351 \mathrm{~b}$ & $228.22 \pm 34.23$ & $655.21 \pm 98.28$ & $2.16 \pm 0.43$ & $389.75 \pm 19.49$ \\
\hline NGC 3521 Enuc. 1 & $14.33 \pm 2.15$ & $18.52 \pm 2.78$ & $0.12 \pm 0.02$ & $6.20 \pm 0.31$ \\
\hline NGC 3521 Enuc. 3 & $9.02 \pm 1.36$ & $15.39 \pm 2.32$ & $0.09 \pm 0.02$ & $6.38 \pm 0.32$ \\
\hline NGC 3521 & $29.66 \pm 4.45$ & $135.30 \pm 20.30$ & $0.59 \pm 0.12$ & $12.56 \pm 0.63$ \\
\hline NGC 3521 Enuc. 2 a & $12.34 \pm 1.86$ & $27.24 \pm 4.09$ & $0.27 \pm 0.05$ & $18.80 \pm 0.94$ \\
\hline NGC 3521 Enuc. 2 b & $6.25 \pm 0.95$ & $12.44 \pm 1.87$ & $0.049 \pm 0.010$ & $2.59 \pm 0.13$ \\
\hline NGC 3621 & $15.55 \pm 2.34$ & $48.94 \pm 7.34$ & $\ldots$ & $7.92 \pm 0.40$ \\
\hline NGC 3627 & $16.12 \pm 2.42$ & $100.99 \pm 15.15$ & $0.93 \pm 0.19$ & $246.46 \pm 12.32$ \\
\hline NGC 3627 Enuc. 1 & $5.43 \pm 0.83$ & $9.08 \pm 1.37$ & $0.38 \pm 0.08$ & $170.02 \pm 8.50$ \\
\hline NGC 3627 Enuc. 2 & $28.07 \pm 4.21$ & $47.67 \pm 7.15$ & $0.94 \pm 0.19$ & $478.65 \pm 23.93$ \\
\hline NGC 3773 & $1163.59 \pm 174.54$ & $1035.24 \pm 155.29$ & $\ldots$ & $57.96 \pm 2.90$ \\
\hline NGC 3938 a & $45.04 \pm 6.76$ & $102.39 \pm 15.37$ & $0.06 \pm 0.01$ & $5.02 \pm 0.25$ \\
\hline NGC 3938 Enuc. 2 a & $76.37 \pm 11.46$ & $80.18 \pm 12.04$ & $0.28 \pm 0.06$ & $7.65 \pm 0.38$ \\
\hline NGC 3938 Enuc. 2 b & $101.00 \pm 15.15$ & $109.29 \pm 16.41$ & $0.35 \pm 0.07$ & $21.20 \pm 1.06$ \\
\hline NGC 4254 Enuc. 2 a & $82.48 \pm 12.37$ & $118.60 \pm 17.79$ & $0.34 \pm 0.07$ & $13.34 \pm 0.67$ \\
\hline NGC 4254 Enuc. 2 b & $79.65 \pm 11.95$ & $122.20 \pm 18.33$ & $0.19 \pm 0.04$ & $11.10 \pm 0.56$ \\
\hline NGC 4254 a & $43.05 \pm 6.46$ & $115.81 \pm 17.37$ & $0.45 \pm 0.09$ & $32.49 \pm 1.62$ \\
\hline NGC 4254 Enuc. 1 a & $35.31 \pm 5.30$ & $53.66 \pm 8.05$ & $0.24 \pm 0.05$ & $22.07 \pm 1.10$ \\
\hline NGC $4254 \mathrm{~b}$ & $41.12 \pm 6.17$ & $160.80 \pm 24.12$ & $0.61 \pm 0.12$ & $53.56 \pm 2.68$ \\
\hline NGC 4254 Enuc. 1 b & $133.40 \pm 20.01$ & $208.80 \pm 31.32$ & $0.53 \pm 0.11$ & $18.13 \pm 0.91$ \\
\hline NGC $4254 \mathrm{c}$ & $38.51 \pm 5.78$ & $114.72 \pm 17.21$ & $0.40 \pm 0.08$ & $25.93 \pm 1.30$ \\
\hline NGC 4254 Enuc. $1 \mathrm{c}$ & $81.14 \pm 12.17$ & $145.03 \pm 21.76$ & $0.50 \pm 0.10$ & $31.93 \pm 1.60$ \\
\hline NGC $4254 \mathrm{~d}$ & $48.25 \pm 7.24$ & $99.19 \pm 14.88$ & $0.24 \pm 0.05$ & $14.27 \pm 0.71$ \\
\hline NGC $4254 \mathrm{e}$ & $74.41 \pm 11.16$ & $134.91 \pm 20.24$ & $0.33 \pm 0.07$ & $25.00 \pm 1.25$ \\
\hline NGC $4254 \mathrm{f}$ & $53.17 \pm 7.98$ & $101.76 \pm 15.26$ & $0.24 \pm 0.05$ & $11.40 \pm 0.57$ \\
\hline
\end{tabular}


Table A1

(Continued)

\begin{tabular}{|c|c|c|c|c|}
\hline Source ID & $\begin{array}{c}f_{\nu}(1528 \AA ̊ \AA) \\
(\mu \mathrm{Jy})\end{array}$ & $\begin{array}{c}f_{\nu}(2271 \AA) \\
(\mu \mathrm{Jy})\end{array}$ & $\begin{array}{c}f_{\mathrm{H} \alpha} / 10^{-13} \\
\left(\mathrm{erg} \mathrm{s}^{-1} \mathrm{~cm}^{-2}\right)\end{array}$ & $\begin{array}{l}f_{\nu}(24 \mu \mathrm{m}) \\
(\mathrm{mJy})\end{array}$ \\
\hline NGC 4321 Enuc. 2 a & $70.84 \pm 10.63$ & $101.18 \pm 15.18$ & $0.15 \pm 0.03$ & $7.99 \pm 0.40$ \\
\hline NGC 4321 Enuc. 2 b & $78.57 \pm 11.79$ & $109.62 \pm 16.44$ & $0.15 \pm 0.03$ & $6.33 \pm 0.32$ \\
\hline NGC 4321 Enuc. 2 & $25.65 \pm 3.85$ & $35.61 \pm 5.34$ & $0.06 \pm 0.01$ & $4.76 \pm 0.24$ \\
\hline NGC $4321 \mathrm{a}$ & $467.33 \pm 70.10$ & $1001.88 \pm 150.28$ & $0.86 \pm 0.17$ & $88.54 \pm 4.43$ \\
\hline NGC 4321 b & $208.14 \pm 31.22$ & $535.72 \pm 80.36$ & $0.90 \pm 0.18$ & $117.01 \pm 5.85$ \\
\hline NGC 4321 Enuc. 1 & $47.40 \pm 7.11$ & $84.43 \pm 12.67$ & $0.10 \pm 0.02$ & $4.10 \pm 0.21$ \\
\hline NGC 4536 & $28.74 \pm 4.32$ & $93.49 \pm 14.04$ & $1.96 \pm 0.39$ & $976.15 \pm 48.81$ \\
\hline NGC 4559 a & $119.11 \pm 17.87$ & $167.28 \pm 25.09$ & $0.77 \pm 0.15$ & $9.99 \pm 0.50$ \\
\hline NGC 4559 b & $199.22 \pm 29.88$ & $258.30 \pm 38.75$ & $0.61 \pm 0.12$ & $5.83 \pm 0.29$ \\
\hline NGC 4559 c & $81.18 \pm 12.18$ & $126.24 \pm 18.94$ & $0.66 \pm 0.13$ & $18.40 \pm 0.92$ \\
\hline NGC 4569 & $330.64 \pm 49.60$ & $1095.12 \pm 164.27$ & $3.27 \pm 0.65$ & $312.76 \pm 15.64$ \\
\hline NGC 4579 & $89.93 \pm 13.49$ & $180.92 \pm 27.14$ & $1.94 \pm 0.39$ & $96.78 \pm 4.84$ \\
\hline NGC 4594 a & $123.89 \pm 18.58$ & $238.40 \pm 35.76$ & $0.82 \pm 0.16$ & $34.38 \pm 1.72$ \\
\hline NGC 4625 & $83.15 \pm 12.47$ & $145.11 \pm 21.77$ & $0.16 \pm 0.03$ & $4.72 \pm 0.24$ \\
\hline NGC 4631 a & $90.42 \pm 13.56$ & $105.16 \pm 15.77$ & $0.38 \pm 0.08$ & $40.73 \pm 2.04$ \\
\hline NGC $4631 \mathrm{~b}$ & $99.88 \pm 14.98$ & $144.01 \pm 21.60$ & $0.48 \pm 0.10$ & $85.41 \pm 4.27$ \\
\hline NGC $4631 \mathrm{c}$ & $144.21 \pm 21.63$ & $183.56 \pm 27.53$ & $0.25 \pm 0.05$ & $167.16 \pm 8.36$ \\
\hline NGC $4631 \mathrm{~d}$ & $61.78 \pm 9.27$ & $92.18 \pm 13.83$ & $0.41 \pm 0.08$ & $45.26 \pm 2.26$ \\
\hline NGC $4631 \mathrm{~h}$ & $50.54 \pm 7.58$ & $68.74 \pm 10.31$ & $0.30 \pm 0.06$ & $268.79 \pm 13.44$ \\
\hline NGC 4631 Enuc. 2 a & $82.13 \pm 12.32$ & $106.50 \pm 15.98$ & $0.67 \pm 0.13$ & $18.86 \pm 0.94$ \\
\hline NGC 4631 Enuc. 2 b & $277.07 \pm 41.56$ & $345.20 \pm 51.78$ & $1.71 \pm 0.34$ & $41.08 \pm 2.05$ \\
\hline NGC 4725 b & $1.91 \pm 0.56$ & $5.85 \pm 1.00$ & $0.013 \pm 0.003$ & $0.30 \pm 0.02$ \\
\hline NGC 4736 & $251.70 \pm 37.75$ & $926.35 \pm 138.95$ & $0.57 \pm 0.11$ & $302.54 \pm 15.13$ \\
\hline NGC 4736 Enuc. $1 \mathrm{a}$ & $330.89 \pm 49.63$ & $438.76 \pm 65.81$ & $0.88 \pm 0.18$ & $83.84 \pm 4.19$ \\
\hline NGC 4736 Enuc. $1 \mathrm{~b}$ & $208.78 \pm 31.32$ & $265.52 \pm 39.83$ & $0.81 \pm 0.16$ & $88.34 \pm 4.42$ \\
\hline NGC 4736 Enuc. $1 \mathrm{c}$ & $197.88 \pm 29.68$ & $257.39 \pm 38.61$ & $0.55 \pm 0.11$ & $90.39 \pm 4.52$ \\
\hline NGC 4826 & $68.74 \pm 10.31$ & $227.33 \pm 34.10$ & $3.67 \pm 0.73$ & $198.25 \pm 9.91$ \\
\hline NGC 5055 & $44.22 \pm 6.63$ & $169.19 \pm 25.38$ & $1.29 \pm 0.26$ & $40.56 \pm 2.03$ \\
\hline NGC 5055 Enuc. 1 & $79.73 \pm 11.96$ & $116.15 \pm 17.42$ & $0.68 \pm 0.14$ & $22.59 \pm 1.13$ \\
\hline NGC 5194 Enuc. 6 a & $47.29 \pm 7.09$ & $57.62 \pm 8.64$ & $0.48 \pm 0.10$ & $25.76 \pm 1.29$ \\
\hline NGC 5194 Enuc. 2 & $276.65 \pm 41.50$ & $425.30 \pm 63.80$ & $1.48 \pm 0.30$ & $98.92 \pm 4.95$ \\
\hline NGC 5194 Enuc. 3 & $320.93 \pm 48.14$ & $548.20 \pm 82.23$ & $0.72 \pm 0.14$ & $60.68 \pm 3.03$ \\
\hline NGC 5194 Enuc. 11 a & $150.83 \pm 22.62$ & $214.00 \pm 32.10$ & $0.46 \pm 0.09$ & $13.72 \pm 0.69$ \\
\hline NGC 5194 Enuc. 11 b & $27.82 \pm 4.17$ & $44.70 \pm 6.70$ & $0.14 \pm 0.03$ & $9.92 \pm 0.50$ \\
\hline NGC 5194 Enuc. $11 \mathrm{~d}$ & $17.34 \pm 2.60$ & $28.96 \pm 4.34$ & $0.12 \pm 0.02$ & $14.66 \pm 0.73$ \\
\hline NGC 5194 Enuc. $11 \mathrm{c}$ & $10.69 \pm 1.60$ & $17.52 \pm 2.63$ & $0.09 \pm 0.02$ & $2.85 \pm 0.14$ \\
\hline NGC 5194 c & $17.54 \pm 2.63$ & $46.97 \pm 7.05$ & $0.33 \pm 0.07$ & $50.31 \pm 2.52$ \\
\hline NGC 5194 b & $97.75 \pm 14.66$ & $240.50 \pm 36.08$ & $0.65 \pm 0.13$ & $36.54 \pm 1.83$ \\
\hline NGC 5194 Enuc. 1 b & $79.39 \pm 11.91$ & $179.50 \pm 26.93$ & $1.02 \pm 0.20$ & $73.51 \pm 3.68$ \\
\hline NGC $5194 \mathrm{e}$ & $135.19 \pm 20.28$ & $341.46 \pm 51.22$ & $0.72 \pm 0.14$ & $30.48 \pm 1.52$ \\
\hline NGC 5194 d & $104.71 \pm 15.71$ & $316.95 \pm 47.54$ & $1.60 \pm 0.32$ & $95.37 \pm 4.77$ \\
\hline NGC 5194 Enuc. 10 a & $15.55 \pm 2.33$ & $31.76 \pm 4.76$ & $0.19 \pm 0.04$ & $19.80 \pm 0.99$ \\
\hline NGC 5194 Enuc. 4 b & $107.63 \pm 16.15$ & $171.40 \pm 25.71$ & $0.017 \pm 0.004$ & $33.66 \pm 1.68$ \\
\hline NGC 5194 Enuc. 4 c & $47.73 \pm 7.16$ & $75.01 \pm 11.25$ & $0.017 \pm 0.004$ & $12.98 \pm 0.65$ \\
\hline NGC 5194 a & $239.15 \pm 35.87$ & $521.05 \pm 78.16$ & $0.63 \pm 0.13$ & $104.98 \pm 5.25$ \\
\hline NGC 5194 Enuc. 10 b & $37.37 \pm 5.61$ & $70.53 \pm 10.58$ & $0.37 \pm 0.07$ & $38.48 \pm 1.92$ \\
\hline NGC 5194 Enuc. 4 d & $117.81 \pm 17.67$ & $208.64 \pm 31.30$ & $0.017 \pm 0.004$ & $22.84 \pm 1.14$ \\
\hline NGC 5194 Enuc. 5 a & $244.35 \pm 36.65$ & $386.49 \pm 57.97$ & $0.017 \pm 0.004$ & $32.37 \pm 1.62$ \\
\hline NGC 5194 Enuc. 9 & $71.73 \pm 10.76$ & $126.37 \pm 18.96$ & $0.37 \pm 0.07$ & $51.39 \pm 2.57$ \\
\hline NGC 5194 Enuc. 7 a & $63.36 \pm 9.50$ & $93.36 \pm 14.00$ & $0.42 \pm 0.08$ & $9.06 \pm 0.45$ \\
\hline NGC 5194 Enuc. 8 & $172.68 \pm 25.90$ & $256.71 \pm 38.51$ & $0.66 \pm 0.13$ & $109.50 \pm 5.48$ \\
\hline NGC 5194 Enuc. 7 b & $142.52 \pm 21.38$ & $217.91 \pm 32.69$ & $1.10 \pm 0.22$ & $50.44 \pm 2.52$ \\
\hline NGC 5194 Enuc. 7 c & $77.98 \pm 11.70$ & $110.21 \pm 16.53$ & $0.37 \pm 0.07$ & $14.50 \pm 0.73$ \\
\hline NGC 5398 & $371.57 \pm 55.74$ & $454.84 \pm 68.23$ & $\ldots$ & $94.86 \pm 4.74$ \\
\hline NGC 5457 Enuc. 6 a & $801.24 \pm 120.19$ & $854.11 \pm 128.12$ & $1.69 \pm 0.34$ & $61.86 \pm 3.09$ \\
\hline NGC 5457 Enuc. 6 b & $414.22 \pm 62.13$ & $511.89 \pm 76.78$ & $1.37 \pm 0.27$ & $56.33 \pm 2.82$ \\
\hline NGC 5457 Enuc. $6 \mathrm{c}$ & $240.17 \pm 36.03$ & $312.87 \pm 46.93$ & $1.22 \pm 0.24$ & $63.55 \pm 3.18$ \\
\hline NGC 5457 Enuc. 5 a & $1012.84 \pm 151.93$ & $1143.52 \pm 171.53$ & $2.53 \pm 0.51$ & $74.87 \pm 3.74$ \\
\hline NGC 5457 Enuc. 1 & $55.52 \pm 8.33$ & $99.52 \pm 14.93$ & $0.21 \pm 0.04$ & $13.01 \pm 0.65$ \\
\hline NGC 5457 & $286.03 \pm 42.90$ & $537.52 \pm 80.63$ & $0.46 \pm 0.09$ & $52.97 \pm 2.65$ \\
\hline NGC 5457 Enuc. 3 a & $94.93 \pm 14.24$ & $112.13 \pm 16.82$ & $0.49 \pm 0.10$ & $15.02 \pm 0.75$ \\
\hline NGC 5457 Enuc. 3 b & $203.99 \pm 30.60$ & $238.33 \pm 35.75$ & $0.98 \pm 0.20$ & $51.61 \pm 2.58$ \\
\hline NGC 5457 Enuc. 3 c & $751.75 \pm 112.76$ & $898.85 \pm 134.83$ & $4.92 \pm 0.98$ & $597.00 \pm 29.85$ \\
\hline
\end{tabular}


Table A1

(Continued)

\begin{tabular}{|c|c|c|c|c|}
\hline Source ID & $\begin{array}{c}f_{\nu}(1528 \AA ̊ \AA) \\
(\mu \mathrm{Jy})\end{array}$ & $\begin{array}{c}f_{\nu}(2271 \AA) \\
(\mu \mathrm{Jy})\end{array}$ & $\begin{array}{c}f_{\mathrm{H} \alpha} / 10^{-13} \\
\left(\mathrm{erg} \mathrm{s}^{-1} \mathrm{~cm}^{-2}\right)\end{array}$ & $\begin{array}{c}f_{\nu}(24 \mu \mathrm{m}) \\
(\mathrm{mJy})\end{array}$ \\
\hline NGC 5457 Enuc. $3 \mathrm{~d}$ & $36.79 \pm 5.52$ & $43.18 \pm 6.48$ & $0.30 \pm 0.06$ & $38.97 \pm 1.95$ \\
\hline NGC 5457 Enuc. 4 a & $357.24 \pm 53.59$ & $375.74 \pm 56.36$ & $0.55 \pm 0.11$ & $7.03 \pm 0.35$ \\
\hline NGC 5457 Enuc. 4 b & $430.15 \pm 64.52$ & $441.69 \pm 66.25$ & $0.58 \pm 0.12$ & $11.24 \pm 0.56$ \\
\hline NGC 5457 Enuc. $4 \mathrm{c}$ & $422.89 \pm 63.43$ & $413.00 \pm 61.95$ & $1.16 \pm 0.23$ & $39.65 \pm 1.98$ \\
\hline NGC 5457 Enuc. $4 \mathrm{~d}$ & $401.84 \pm 60.28$ & $432.77 \pm 64.92$ & $1.17 \pm 0.23$ & $26.85 \pm 1.34$ \\
\hline NGC 5474 & $63.65 \pm 9.55$ & $97.97 \pm 14.70$ & $0.10 \pm 0.02$ & $1.23 \pm 0.06$ \\
\hline NGC 5713 Enuc. 2 a & $157.17 \pm 23.58$ & $254.86 \pm 38.23$ & $0.39 \pm 0.08$ & $57.55 \pm 2.88$ \\
\hline NGC 5713 Enuc. 2 b & $36.34 \pm 5.45$ & $72.06 \pm 10.81$ & $0.06 \pm 0.01$ & $32.09 \pm 1.60$ \\
\hline NGC 5713 & $91.36 \pm 13.70$ & $208.13 \pm 31.22$ & $0.06 \pm 0.01$ & $54.73 \pm 2.74$ \\
\hline NGC 5713 Enuc. 1 & $123.22 \pm 18.48$ & $322.24 \pm 48.34$ & $0.09 \pm 0.02$ & $364.30 \pm 18.21$ \\
\hline NGC 5866 & $12.80 \pm 1.93$ & $48.21 \pm 7.24$ & $\ldots$ & $19.91 \pm 1.00$ \\
\hline NGC 6946 Enuc. 4 b & $5.65 \pm 0.99$ & $9.19 \pm 1.41$ & $\cdots$ & $1.06 \pm 0.05$ \\
\hline NGC 6946 Enuc. $4 \mathrm{c}$ & $5.45 \pm 0.97$ & $11.02 \pm 1.68$ & $\ldots$ & $121.25 \pm 6.06$ \\
\hline NGC 6946 Enuc. 8 & $6.02 \pm 1.04$ & $9.83 \pm 1.51$ & $1.34 \pm 0.27$ & $78.79 \pm 3.94$ \\
\hline NGC 6946 Enuc. 5 a & $8.29 \pm 1.35$ & $11.09 \pm 1.69$ & $0.62 \pm 0.12$ & $4.21 \pm 0.21$ \\
\hline NGC 6946 Enuc. 3 a & $7.23 \pm 1.20$ & $9.65 \pm 1.48$ & $1.14 \pm 0.23$ & $5.22 \pm 0.26$ \\
\hline NGC 6946 a & $20.27 \pm 3.08$ & $37.11 \pm 5.58$ & $1.58 \pm 0.32$ & $70.92 \pm 3.55$ \\
\hline NGC 6946 c & $6.61 \pm 1.12$ & $13.80 \pm 2.09$ & $0.97 \pm 0.19$ & $43.93 \pm 2.20$ \\
\hline NGC 6946 Enuc. 6 a & $14.49 \pm 2.23$ & $22.62 \pm 3.41$ & $2.86 \pm 0.57$ & $103.34 \pm 5.17$ \\
\hline NGC 6946 Enuc. 6 b & $8.41 \pm 1.36$ & $9.23 \pm 1.42$ & $1.02 \pm 0.20$ & $42.42 \pm 2.12$ \\
\hline NGC 6946 Enuc. 7 & $9.88 \pm 1.57$ & $13.28 \pm 2.02$ & $1.13 \pm 0.23$ & $71.13 \pm 3.56$ \\
\hline NGC 6946 Enuc. 2 b & $134.48 \pm 20.18$ & $173.55 \pm 26.03$ & $8.20 \pm 1.64$ & $67.97 \pm 3.40$ \\
\hline NGC 7331 & $8.70 \pm 1.31$ & $53.98 \pm 8.10$ & $<0.20$ & $16.82 \pm 0.84$ \\
\hline NGC 7793 Enuc. 1 & $384.10 \pm 57.61$ & $381.23 \pm 57.18$ & $0.64 \pm 0.13$ & $5.09 \pm 0.25$ \\
\hline NGC 7793 Enuc. 3 & $291.93 \pm 43.79$ & $357.16 \pm 53.57$ & $0.68 \pm 0.14$ & $25.96 \pm 1.30$ \\
\hline NGC 7793 a & $201.40 \pm 30.21$ & $286.53 \pm 42.98$ & $0.58 \pm 0.12$ & $10.60 \pm 0.53$ \\
\hline NGC 7793 b & $313.42 \pm 47.01$ & $462.00 \pm 69.30$ & $0.69 \pm 0.14$ & $15.49 \pm 0.77$ \\
\hline NGC 7793 Enuc. 2 & $37.06 \pm 5.56$ & $45.85 \pm 6.88$ & $0.22 \pm 0.04$ & $5.59 \pm 0.28$ \\
\hline \multicolumn{5}{|c|}{ Likely Associated with Supernovae } \\
\hline NGC 6946 Enuc. 5 b & $24.28 \pm 3.68$ & $31.79 \pm 4.78$ & $2.18 \pm 0.44$ & $10.55 \pm 0.53$ \\
\hline \multicolumn{5}{|c|}{ Likely AME Candidates } \\
\hline NGC 2403 Enuc. 5 & $1061.51 \pm 159.23$ & $1026.40 \pm 153.96$ & $2.31 \pm 0.46$ & $52.86 \pm 2.64$ \\
\hline NGC 2403 Enuc. 3 & $1405.70 \pm 210.85$ & $1561.28 \pm 234.19$ & $4.83 \pm 0.97$ & $284.47 \pm 14.22$ \\
\hline NGC 3938 b & $29.22 \pm 4.39$ & $68.29 \pm 10.26$ & $0.07 \pm 0.01$ & $3.38 \pm 0.17$ \\
\hline NGC 4631 Enuc. 1 & $305.75 \pm 45.86$ & $392.26 \pm 58.84$ & $0.79 \pm 0.16$ & $7.55 \pm 0.38$ \\
\hline NGC $4631 \mathrm{e}$ & $106.11 \pm 15.92$ & $154.92 \pm 23.24$ & $0.45 \pm 0.09$ & $92.84 \pm 4.64$ \\
\hline NGC $4631 \mathrm{f}$ & $80.71 \pm 12.11$ & $114.54 \pm 17.18$ & $0.38 \pm 0.08$ & $74.10 \pm 3.70$ \\
\hline NGC $4631 \mathrm{~g}$ & $65.91 \pm 9.89$ & $88.30 \pm 13.25$ & $0.34 \pm 0.07$ & $143.47 \pm 7.17$ \\
\hline NGC 4725a & $39.11 \pm 5.89$ & $82.23 \pm 12.34$ & $<0.01$ & $21.29 \pm 1.06$ \\
\hline NGC 5194 Enuc. 1 a & $17.13 \pm 2.57$ & $34.57 \pm 5.19$ & $0.55 \pm 0.11$ & $81.10 \pm 4.06$ \\
\hline NGC 5194 Enuc. $11 \mathrm{e}$ & $14.96 \pm 2.24$ & $27.54 \pm 4.13$ & $0.25 \pm 0.05$ & $23.20 \pm 1.16$ \\
\hline NGC 5194 Enuc. $1 \mathrm{c}$ & $40.12 \pm 6.02$ & $95.83 \pm 14.37$ & $0.68 \pm 0.14$ & $32.22 \pm 1.61$ \\
\hline NGC 5194 Enuc. 4 a & $30.59 \pm 4.59$ & $41.93 \pm 6.29$ & $0.018 \pm 0.004$ & $10.45 \pm 0.52$ \\
\hline NGC 5457 Enuc. 2 & $237.86 \pm 35.68$ & $314.69 \pm 47.20$ & $0.68 \pm 0.14$ & $24.21 \pm 1.21$ \\
\hline NGC 5457 Enuc. 7 & $<0.26$ & $<0.46$ & $3.43 \pm 0.69$ & $53.60 \pm 2.68$ \\
\hline NGC 6946 Enuc. 4 a & $33.30 \pm 5.02$ & $47.68 \pm 7.16$ & $\ldots$ & $17.07 \pm 0.85$ \\
\hline NGC 6946 Enuc. 3 b & $42.01 \pm 6.32$ & $51.32 \pm 7.70$ & $2.24 \pm 0.45$ & $21.79 \pm 1.09$ \\
\hline NGC 6946b & $4.34 \pm 0.83$ & $11.18 \pm 1.71$ & $4.60 \pm 0.92$ & $2075.43 \pm 103.77$ \\
\hline NGC 6946 Enuc. 9 & $23.67 \pm 3.59$ & $36.83 \pm 5.53$ & $3.46 \pm 0.69$ & $115.07 \pm 5.75$ \\
\hline NGC 6946 Enuc. 1 & $29.23 \pm 4.41$ & $38.37 \pm 5.76$ & $2.83 \pm 0.57$ & $46.55 \pm 2.33$ \\
\hline NGC 6946 Enuc. 2 a & $31.82 \pm 4.80$ & $41.20 \pm 6.19$ & $2.37 \pm 0.47$ & $17.27 \pm 0.86$ \\
\hline
\end{tabular}

Notes.

${ }^{a}$ Photometry unreliable and not reported owing to saturation by the nucleus in the $24 \mu \mathrm{m}$ image. 
Table A2

Local Background Measurements

\begin{tabular}{|c|c|c|c|c|c|c|}
\hline Source ID & $\begin{array}{c}\text { Back } 3 \mathrm{GHz} \\
(\mathrm{mJy})\end{array}$ & $\begin{array}{c}\text { Back } 15 \mathrm{GHz} \\
(\mathrm{mJy})\end{array}$ & $\begin{array}{c}\text { Back } 33 \mathrm{GHz} \\
(\mathrm{mJy})\end{array}$ & $f_{\text {Back }}^{3 \mathrm{GHz} \text { a }}$ & $f_{\mathrm{Back}}^{15 \mathrm{GHz} \text { a }}$ & $f_{\text {Back }}^{33 \mathrm{GHz} \text { a }}$ \\
\hline \multicolumn{7}{|c|}{ Star-forming Regions } \\
\hline NGC $0337 \mathrm{D}$ & 0.207 & 0.099 & 0.019 & 0.174 & 0.130 & 0.062 \\
\hline NGC 0337 A & 0.046 & 0.070 & 0.024 & 0.046 & 0.100 & 0.085 \\
\hline NGC 0337 B & 0.133 & 0.059 & -0.022 & 0.027 & 0.022 & 0.012 \\
\hline NGC $0337 \mathrm{C}$ & 0.026 & 0.019 & 0.005 & 0.087 & 0.064 & 0.024 \\
\hline NGC 0337 G & 0.030 & 0.013 & -0.005 & 0.082 & 0.068 & 0.032 \\
\hline NGC $0337 \mathrm{E}$ & 0.026 & 0.027 & 0.010 & 0.096 & 0.099 & 0.034 \\
\hline NGC $0337 \mathrm{~F}$ & 0.021 & 0.038 & 0.023 & 0.075 & 0.195 & 0.104 \\
\hline NGC 0628 Enuc. 4 & -0.004 & 0.013 & 0.013 & 0.015 & 0.049 & 0.057 \\
\hline NGC 0628 Enuc. 2 & 0.017 & -0.012 & 0.026 & 0.033 & 0.033 & 0.092 \\
\hline NGC 0628 Enuc. $3 \mathrm{C}$ & 0.009 & 0.010 & 0.018 & 0.033 & 0.050 & 0.080 \\
\hline NGC 0628 Enuc. $3 \mathrm{~A}^{\mathrm{b}}$ & -0.007 & 0.019 & -0.012 & 0.013 & 0.052 & 0.024 \\
\hline NGC 0628 Enuc. 3 B & 0.023 & 0.018 & 0.014 & 0.080 & 0.091 & 0.066 \\
\hline NGC 0628 Enuc. $1 \mathrm{~B}$ & $\ldots$ & 0.063 & $\ldots$ & $\cdots$ & 0.156 & $\cdots$ \\
\hline NGC 0628 Enuc. 1 A & 0.007 & 0.029 & -0.031 & 0.026 & 0.114 & 0.118 \\
\hline NGC $0855 \mathrm{C}$ & 0.082 & 0.071 & 0.018 & 0.045 & 0.067 & 0.024 \\
\hline NGC $0855 \mathrm{~A}^{\mathrm{b}}$ & 0.057 & 0.037 & -0.042 & 0.025 & 0.024 & 0.049 \\
\hline NGC 0855 B & 0.022 & 0.026 & 0.022 & 0.060 & 0.110 & 0.120 \\
\hline NGC 0925 D & -0.004 & 0.005 & $\ldots$ & 0.014 & 0.022 & $\ldots$ \\
\hline NGC 0925 A & 0.020 & $\ldots$ & $\ldots$ & 0.211 & $\ldots$ & $\ldots$ \\
\hline NGC 0925 B & -0.001 & -0.003 & $\cdots$ & 0.004 & 0.019 & $\cdots$ \\
\hline NGC 1097 Enuc. 2 & 0.005 & $\ldots$ & 0.000 & 0.004 & $\ldots$ & 0.002 \\
\hline NGC 1097 D & 1.142 & 0.248 & 0.223 & 0.096 & 0.083 & 0.100 \\
\hline NGC 1097 B & 2.604 & 0.681 & 0.481 & 0.367 & 0.203 & 0.159 \\
\hline NGC $1097 \mathrm{~A}^{\mathrm{b}}$ & 5.145 & 0.574 & 0.810 & 0.049 & 0.020 & 0.038 \\
\hline NGC $1097 \mathrm{E}$ & 1.014 & 0.248 & 0.180 & 0.103 & 0.084 & 0.095 \\
\hline NGC 1097 C & 1.450 & 0.285 & 0.235 & 0.119 & 0.087 & 0.100 \\
\hline NGC 1097 Enuc. 1 & $\ldots$ & $\ldots$ & 0.023 & $\ldots$ & $\ldots$ & 0.073 \\
\hline NGC $1266 \mathrm{C}$ & 0.089 & 0.039 & 0.051 & 0.003 & 0.004 & 0.009 \\
\hline NGC $1266 \mathrm{~A}^{\mathrm{b}}$ & 0.079 & 0.006 & 0.037 & 0.002 & 0.001 & 0.009 \\
\hline NGC 1266 B & 0.121 & 0.031 & 0.014 & 0.006 & 0.007 & 0.006 \\
\hline NGC 1377 & 0.003 & -0.005 & 0.004 & 0.014 & 0.073 & 0.069 \\
\hline IC $342 \mathrm{~B}$ & 3.176 & 1.428 & 1.135 & 0.081 & 0.078 & 0.081 \\
\hline IC $342 \mathrm{~A}^{\mathrm{b}}$ & 2.376 & 0.743 & 0.477 & 0.026 & 0.020 & 0.018 \\
\hline IC $342 \mathrm{C}$ & 2.542 & 0.970 & 0.790 & 0.109 & 0.093 & 0.098 \\
\hline IC 342 D & 0.077 & 0.027 & $\ldots$ & 0.086 & 0.199 & $\ldots$ \\
\hline NGC $1482 \mathrm{C}$ & 1.810 & 0.339 & 0.237 & 0.039 & 0.026 & 0.026 \\
\hline NGC $1482 \mathrm{~A}^{\mathrm{b}}$ & 1.692 & 0.078 & 0.255 & 0.014 & 0.003 & 0.023 \\
\hline NGC 1482 B & 1.855 & 0.606 & 0.270 & 0.043 & 0.048 & 0.031 \\
\hline NGC 2146 A & 0.855 & 0.020 & 0.101 & 0.139 & 0.045 & 0.044 \\
\hline NGC 2146 B & 1.934 & 0.046 & 0.377 & 0.106 & 0.040 & 0.065 \\
\hline NGC $2146 \mathrm{C}$ & 4.865 & 0.136 & 0.971 & 0.132 & 0.081 & 0.097 \\
\hline NGC 2146 D & 16.740 & -0.052 & 1.332 & 0.067 & 0.002 & 0.022 \\
\hline NGC 2146 E & 2.349 & 0.122 & 0.290 & 0.173 & 0.210 & 0.081 \\
\hline NGC 2403 Enuc. 5 C & 0.066 & 0.080 & 0.049 & 0.072 & 0.107 & 0.066 \\
\hline NGC 2403 Enuc. $5 \mathrm{~A}^{\mathrm{b}}$ & 0.014 & 0.114 & 0.045 & 0.007 & 0.055 & 0.025 \\
\hline NGC 2403 Enuc. 5 E & 0.004 & 0.012 & $\cdots$ & 0.018 & 0.052 & $\cdots$ \\
\hline NGC 2403 Enuc. 5 B & 0.004 & 0.006 & 0.006 & 0.026 & 0.033 & 0.059 \\
\hline NGC 2403 Enuc. 6 A & -0.023 & 0.026 & 0.008 & 0.026 & 0.030 & 0.011 \\
\hline NGC 2403 Enuc. 6 B & 0.015 & $\cdots$ & 0.000 & 0.130 & $\cdots$ & 0.002 \\
\hline NGC 2403 Enuc. $1 \mathrm{E}$ & 0.014 & 0.010 & $\ldots$ & 0.058 & 0.035 & $\ldots$ \\
\hline NGC 2403 Enuc. $1 \mathrm{~A}$ & -0.001 & 0.013 & -0.001 & 0.001 & 0.033 & 0.003 \\
\hline NGC $2403 \mathrm{C}$ & 0.002 & 0.012 & $\cdots$ & 0.017 & 0.091 & $\cdots$ \\
\hline NGC 2403 Enuc. $1 \mathrm{~B}$ & 0.031 & 0.066 & 0.040 & 0.020 & 0.048 & 0.035 \\
\hline NGC 2403 Enuc. $1 \mathrm{C}$ & 0.036 & 0.005 & 0.003 & 0.076 & 0.048 & 0.035 \\
\hline NGC 2403 Enuc. 1 D & 0.022 & 0.023 & 0.009 & 0.108 & 0.170 & 0.079 \\
\hline NGC 2403 Enuc. 2 B & -0.008 & 0.018 & -0.002 & 0.020 & 0.055 & 0.009 \\
\hline NGC 2403 Enuc. 2 D & 0.039 & 0.030 & 0.029 & 0.061 & 0.054 & 0.053 \\
\hline NGC 2403 Enuc. $2 \mathrm{~A}^{\mathrm{b}}$ & 0.024 & 0.024 & 0.061 & 0.011 & 0.015 & 0.041 \\
\hline NGC 2403 Enuc. 2 C & 0.050 & 0.025 & 0.022 & 0.084 & 0.055 & 0.055 \\
\hline NGC 2403 B & 0.027 & $\cdots$ & $\cdots$ & 0.138 & $\cdots$ & $\cdots$ \\
\hline NGC 2403 E & 0.013 & $\ldots$ & $\ldots$ & 0.096 & $\ldots$ & $\cdots$ \\
\hline NGC 2403 Enuc. 3 B & 0.177 & 0.165 & 0.229 & 0.046 & 0.052 & 0.079 \\
\hline
\end{tabular}


Table A2

(Continued)

\begin{tabular}{|c|c|c|c|c|c|c|}
\hline Source ID & $\begin{array}{l}\text { Back } 3 \mathrm{GHz} \\
(\mathrm{mJy})\end{array}$ & $\begin{array}{l}\text { Back } 15 \mathrm{GHz} \\
(\mathrm{mJy})\end{array}$ & $\begin{array}{l}\text { Back } 33 \mathrm{GHz} \\
(\mathrm{mJy})\end{array}$ & $f_{\text {Back }}^{3 \mathrm{GHz} \text { a }}$ & $f_{\mathrm{Back}}^{15 \mathrm{GHz} a}$ & $f_{\text {Back }}^{33 \mathrm{GHz} \text { a }}$ \\
\hline NGC 2403 Enuc. 4 B & $\ldots$ & 0.018 & 0.003 & $\ldots$ & 0.158 & 0.039 \\
\hline NGC 2403 Enuc. 4 C & $\ldots$ & 0.013 & 0.008 & $\ldots$ & 0.122 & 0.098 \\
\hline NGC 2403 Enuc. 4 A & 0.016 & 0.066 & 0.034 & 0.016 & 0.066 & 0.037 \\
\hline Holmberg II B & 0.006 & 0.011 & 0.019 & 0.041 & 0.080 & 0.115 \\
\hline Holmberg II A & 0.017 & 0.016 & 0.031 & 0.050 & 0.050 & 0.091 \\
\hline NGC 2798 B & 0.180 & 0.041 & 0.011 & 0.102 & 0.067 & 0.057 \\
\hline NGC 2798 A & 0.624 & 0.184 & 0.058 & 0.056 & 0.073 & 0.062 \\
\hline NGC 2841 A & -0.003 & $\ldots$ & $\ldots$ & 0.005 & $\ldots$ & $\ldots$ \\
\hline NGC 2976 Enuc. $1 \mathrm{~A}$ & 0.043 & 0.044 & 0.032 & 0.038 & 0.057 & 0.043 \\
\hline NGC 2976 Enuc. 1 D & 0.143 & 0.101 & 0.073 & 0.064 & 0.056 & 0.047 \\
\hline NGC 2976 Enuc. $1 \mathrm{C}$ & 0.019 & 0.019 & 0.019 & 0.039 & 0.052 & 0.059 \\
\hline NGC 2976 Enuc. $1 \mathrm{~B}^{\mathrm{b}}$ & 0.106 & 0.145 & 0.063 & 0.032 & 0.058 & 0.031 \\
\hline NGC 2976 B & 0.014 & 0.004 & -0.001 & 0.086 & 0.025 & 0.005 \\
\hline NGC 2976 A & $\ldots$ & -0.004 & $\ldots$ & $\ldots$ & 0.027 & $\ldots$ \\
\hline NGC 2976 Enuc. 2 A & 0.069 & 0.068 & 0.025 & 0.041 & 0.046 & 0.020 \\
\hline NGC 2976 Enuc. 2 C & 0.047 & 0.045 & 0.015 & 0.084 & 0.099 & 0.038 \\
\hline NGC 2976 C & 0.012 & $\ldots$ & $\ldots$ & 0.048 & $\ldots$ & $\ldots$ \\
\hline NGC 3049 A & 0.007 & 0.021 & 0.018 & 0.005 & 0.039 & 0.040 \\
\hline NGC 3049 B & 0.005 & 0.014 & 0.014 & 0.015 & 0.066 & 0.090 \\
\hline NGC 3049 C & 0.009 & $\ldots$ & $\ldots$ & 0.041 & $\ldots$ & $\ldots$ \\
\hline NGC 3077 A & 0.523 & 0.387 & 0.170 & 0.046 & 0.054 & 0.027 \\
\hline NGC $3190 \mathrm{~A}$ & 0.024 & 0.008 & 0.005 & 0.043 & 0.013 & 0.004 \\
\hline NGC 3184 B & 0.013 & 0.012 & $\ldots$ & 0.040 & 0.062 & $\ldots$ \\
\hline NGC 3184 A & 0.027 & -0.006 & -0.004 & 0.042 & 0.018 & 0.012 \\
\hline NGC 3198 B & 0.014 & 0.001 & $\ldots$ & 0.067 & 0.005 & $\ldots$ \\
\hline NGC 3198 A & 0.020 & 0.013 & -0.023 & 0.041 & 0.044 & 0.120 \\
\hline NGC 3198 C & -0.019 & 0.015 & $\ldots$ & 0.112 & 0.085 & $\ldots$ \\
\hline IC 2574 D & 0.032 & 0.016 & 0.010 & 0.033 & 0.053 & 0.021 \\
\hline IC $2574 \mathrm{C}$ & 0.160 & 0.112 & 0.060 & 0.179 & 0.178 & 0.077 \\
\hline IC $2574 \mathrm{~A}$ & 0.009 & 0.004 & 0.011 & 0.016 & 0.008 & 0.023 \\
\hline IC $2574 \mathrm{~B}$ & -0.005 & 0.005 & $\ldots$ & 0.056 & 0.049 & $\ldots$ \\
\hline NGC 3265 A & 0.145 & 0.044 & 0.028 & 0.053 & 0.066 & 0.066 \\
\hline NGC $3351 \mathrm{C}$ & -0.016 & -0.022 & -0.010 & 0.007 & 0.021 & 0.008 \\
\hline NGC 3351 A & 0.497 & 0.179 & 0.036 & 0.069 & 0.065 & 0.018 \\
\hline NGC 3351 B & 0.409 & 0.101 & 0.109 & 0.046 & 0.028 & 0.048 \\
\hline NGC 3521 Enuc. 1 & -0.022 & 0.023 & $\ldots$ & 0.162 & 0.080 & $\ldots$ \\
\hline NGC 3521 Enuc. 3 A & $\ldots$ & 0.027 & $\ldots$ & $\ldots$ & 0.085 & $\ldots$ \\
\hline NGC 3521 Enuc. 2 A & 0.021 & 0.016 & -0.019 & 0.032 & 0.047 & 0.068 \\
\hline NGC 3521 Enuc. 3 C & 0.021 & $\ldots$ & $\ldots$ & 0.021 & $\ldots$ & $\ldots$ \\
\hline NGC 3521 Enuc. 2 B & 0.011 & 0.012 & $\ldots$ & 0.037 & 0.034 & $\ldots$ \\
\hline NGC $3621 \mathrm{C}$ & 0.025 & 0.032 & $\ldots$ & 0.065 & 0.099 & $\ldots$ \\
\hline NGC 3621 A & 0.060 & 0.026 & $\ldots$ & 0.057 & 0.095 & $\ldots$ \\
\hline NGC $3621 \mathrm{H}$ & 0.012 & $\ldots$ & $\ldots$ & 0.103 & $\ldots$ & $\ldots$ \\
\hline NGC $3621 \mathrm{G}^{\mathrm{b}}$ & 0.109 & 0.079 & $\ldots$ & 0.051 & 0.051 & $\ldots$ \\
\hline NGC 3621 B & 0.035 & 0.048 & $\cdots$ & 0.037 & 0.086 & $\cdots$ \\
\hline NGC $3621 \mathrm{~F}$ & 0.002 & $\ldots$ & $\ldots$ & 0.013 & $\ldots$ & $\ldots$ \\
\hline NGC $3621 \mathrm{E}$ & -0.001 & -0.010 & $\ldots$ & 0.002 & 0.054 & $\ldots$ \\
\hline NGC 3627 Enuc. $1 \mathrm{G}$ & 0.037 & $\ldots$ & $\ldots$ & 0.072 & $\ldots$ & $\ldots$ \\
\hline NGC 3627 Enuc. $1 \mathrm{~F}$ & 0.015 & $\ldots$ & $\ldots$ & 0.064 & $\ldots$ & $\ldots$ \\
\hline NGC 3627 Enuc. $1 \mathrm{E}$ & -0.002 & $\ldots$ & 0.003 & 0.006 & $\ldots$ & 0.007 \\
\hline NGC 3627 B & 0.382 & 0.392 & -0.004 & 0.096 & 0.162 & 0.003 \\
\hline NGC 3627 A & 0.008 & 0.037 & 0.021 & 0.004 & 0.034 & 0.030 \\
\hline NGC 3627 Enuc. 2 D & 0.140 & 0.073 & 0.060 & 0.034 & 0.029 & 0.032 \\
\hline NGC 3627 Enuc. $1 \mathrm{~A}$ & 0.074 & 0.118 & 0.035 & 0.031 & 0.081 & 0.028 \\
\hline NGC 3627 Enuc. $2 \mathrm{~A}^{\mathrm{b}}$ & 0.161 & 0.208 & 0.029 & 0.022 & 0.049 & 0.008 \\
\hline NGC 3627 Enuc. 2 E & 0.197 & 0.120 & 0.077 & 0.048 & 0.057 & 0.046 \\
\hline NGC 3627 Enuc. 2 C & -0.008 & 0.039 & 0.003 & 0.019 & 0.092 & 0.012 \\
\hline NGC 3627 Enuc. 2 B & 0.027 & 0.059 & $\ldots$ & 0.035 & 0.117 & $\ldots$ \\
\hline NGC 3773 & 0.078 & 0.049 & 0.017 & 0.031 & 0.036 & 0.015 \\
\hline NGC 3938 B & 0.028 & $\ldots$ & $\ldots$ & 0.102 & $\ldots$ & $\ldots$ \\
\hline NGC 3938 Enuc. 2 B & 0.002 & -0.003 & 0.011 & 0.009 & 0.027 & 0.060 \\
\hline NGC 3938 Enuc. 2 A & -0.007 & 0.000 & -0.003 & 0.021 & 0.000 & 0.013 \\
\hline NGC 4254 Enuc. 2 D & $\ldots$ & 0.008 & 0.019 & $\ldots$ & 0.081 & 0.112 \\
\hline
\end{tabular}


Table A2

(Continued)

\begin{tabular}{|c|c|c|c|c|c|c|}
\hline Source ID & $\begin{array}{l}\text { Back } 3 \mathrm{GHz} \\
(\mathrm{mJy})\end{array}$ & $\begin{array}{l}\text { Back } 15 \mathrm{GHz} \\
(\mathrm{mJy})\end{array}$ & $\begin{array}{l}\text { Back } 33 \mathrm{GHz} \\
(\mathrm{mJy})\end{array}$ & $f_{\mathrm{Back}}^{3 \mathrm{GHz} \text { a }}$ & $f_{\mathrm{Back}}^{15 \mathrm{GHz} \text { a }}$ & $f_{\mathrm{Back}}^{33 \mathrm{GHz} \text { a }}$ \\
\hline NGC 4254 Enuc. $2 \mathrm{C}$ & $\cdots$ & $\ldots$ & 0.007 & $\cdots$ & $\ldots$ & 0.089 \\
\hline NGC 4254 Enuc. $1 \mathrm{E}$ & 0.020 & 0.013 & $\cdots$ & 0.068 & 0.114 & $\cdots$ \\
\hline NGC 4254 Enuc. 2 E & -0.003 & $\cdots$ & $\cdots$ & 0.019 & $\cdots$ & $\cdots$ \\
\hline NGC 4254 & 0.024 & 0.095 & 0.014 & 0.059 & 0.185 & 0.038 \\
\hline NGC 4254 Enuc. $1 \mathrm{D}$ & 0.056 & 0.011 & 0.014 & 0.071 & 0.046 & 0.040 \\
\hline NGC 4254 Enuc. $1 \mathrm{~B}$ & 0.033 & -0.004 & 0.023 & 0.152 & 0.028 & 0.056 \\
\hline NGC 4254 Enuc. $1 \mathrm{C}$ & 0.002 & $\ldots$ & -0.008 & 0.008 & $\cdots$ & 0.063 \\
\hline NGC 4321 Enuc. 2 & 0.014 & $\cdots$ & -0.010 & 0.082 & $\cdots$ & 0.084 \\
\hline NGC $4321 \mathrm{C}$ & 0.424 & 0.192 & 0.101 & 0.165 & 0.176 & 0.173 \\
\hline NGC $4321 \mathrm{~F}$ & 0.215 & 0.099 & 0.067 & 0.231 & 0.218 & 0.214 \\
\hline NGC $4321 \mathrm{H}$ & 0.115 & 0.055 & 0.037 & 0.176 & 0.177 & 0.136 \\
\hline NGC $4321 \mathrm{~A}^{\mathrm{b}}$ & 0.326 & 0.381 & -0.122 & 0.014 & 0.042 & 0.024 \\
\hline NGC 4321 B & 0.379 & 0.153 & 0.089 & 0.153 & 0.193 & 0.197 \\
\hline NGC $4321 \mathrm{G}$ & 0.330 & 0.115 & 0.076 & 0.186 & 0.184 & 0.198 \\
\hline NGC $4321 \mathrm{D}$ & 0.387 & 0.173 & 0.129 & 0.119 & 0.135 & 0.156 \\
\hline NGC $4321 \mathrm{E}$ & 0.750 & 0.277 & 0.158 & 0.190 & 0.199 & 0.182 \\
\hline NGC 4321 Enuc. $1 \mathrm{~B}$ & -0.012 & 0.008 & $\ldots$ & 0.043 & 0.045 & $\ldots$ \\
\hline NGC $4536 \mathrm{C}$ & 2.914 & 0.834 & 0.455 & 0.086 & 0.066 & 0.057 \\
\hline NGC $4536 \mathrm{~A}^{\mathrm{b}}$ & 1.452 & 0.485 & 0.149 & 0.016 & 0.017 & 0.009 \\
\hline NGC $4536 \mathrm{~B}$ & 2.138 & 0.635 & 0.335 & 0.079 & 0.064 & 0.051 \\
\hline NGC $4559 \mathrm{E}$ & -0.017 & 0.013 & $\ldots$ & 0.116 & 0.106 & $\ldots$ \\
\hline NGC $4559 \mathrm{C}$ & -0.013 & 0.028 & 0.005 & 0.065 & 0.158 & 0.026 \\
\hline NGC $4559 \mathrm{D}$ & $\ldots$ & 0.026 & $\ldots$ & $\ldots$ & 0.228 & $\ldots$ \\
\hline NGC 4559 B & 0.012 & 0.010 & 0.002 & 0.130 & 0.064 & 0.018 \\
\hline NGC 4559 A & 0.002 & 0.008 & 0.002 & 0.014 & 0.073 & 0.013 \\
\hline NGC 4569 A & 0.253 & 0.180 & 0.057 & 0.088 & 0.125 & 0.065 \\
\hline NGC 4579 & -0.139 & 0.254 & 0.152 & 0.068 & 0.092 & 0.255 \\
\hline NGC 4594 & -0.021 & 0.011 & 0.021 & 0.025 & 0.023 & 0.026 \\
\hline NGC 4631 Enuc. 1 & -0.000 & 0.064 & 0.049 & 0.000 & 0.097 & 0.080 \\
\hline NGC $4625 \mathrm{~A}$ & $\ldots$ & 0.035 & $\ldots$ & $\ldots$ & 0.323 & $\ldots$ \\
\hline NGC 4631 A & 0.044 & 0.178 & 0.034 & 0.006 & 0.072 & 0.023 \\
\hline NGC $4631 \mathrm{H}$ & 0.128 & 0.195 & 0.071 & 0.130 & 0.149 & 0.084 \\
\hline NGC 4631 B & 0.456 & 0.220 & 0.208 & 0.083 & 0.066 & 0.068 \\
\hline NGC $4631 \mathrm{G}$ & 0.043 & 0.050 & 0.030 & 0.047 & 0.068 & 0.049 \\
\hline NGC 4631 Enuc. 2 B & 0.014 & 0.042 & -0.000 & 0.009 & 0.030 & 0.000 \\
\hline NGC 4631 Enuc. $2 \mathrm{C}$ & $\cdots$ & 0.019 & $\cdots$ & $\cdots$ & 0.088 & $\cdots$ \\
\hline NGC $4725 \mathrm{C}$ & -0.007 & 0.000 & $\ldots$ & 0.032 & 0.006 & $\cdots$ \\
\hline NGC $4725 \mathrm{~A}$ & 0.003 & 0.002 & 0.001 & 0.038 & 0.014 & 0.002 \\
\hline NGC $4736 \mathrm{~K}$ & 0.004 & 0.015 & $\cdots$ & 0.007 & 0.020 & $\cdots$ \\
\hline NGC $4736 \mathrm{~J}$ & 0.104 & 0.001 & $\cdots$ & 0.103 & 0.002 & $\cdots$ \\
\hline NGC 4736 I & 0.097 & 0.034 & $\cdots$ & 0.077 & 0.052 & $\ldots$ \\
\hline NGC $4736 \mathrm{G}$ & 0.044 & $\ldots$ & $\ldots$ & 0.163 & $\ldots$ & $\ldots$ \\
\hline NGC 4736L & 0.047 & $\ldots$ & $\cdots$ & 0.084 & $\ldots$ & $\ldots$ \\
\hline NGC $4736 \mathrm{H}$ & 0.088 & 0.080 & 0.026 & 0.069 & 0.074 & 0.074 \\
\hline NGC $4736 \mathrm{M}$ & -0.016 & $\ldots$ & $\ldots$ & 0.103 & $\ldots$ & $\ldots$ \\
\hline NGC $4736 \mathrm{~F}$ & 0.134 & 0.108 & $\ldots$ & 0.099 & 0.121 & $\ldots$ \\
\hline NGC 4736N & -0.004 & 0.133 & $\ldots$ & 0.017 & 0.124 & $\ldots$ \\
\hline NGC 4736 A & 0.193 & -0.016 & 0.021 & 0.031 & 0.007 & 0.017 \\
\hline NGC $4736 \mathrm{O}$ & 0.015 & 0.086 & 0.024 & 0.084 & 0.194 & 0.062 \\
\hline NGC $4736 \mathrm{E}$ & $\ldots$ & 0.141 & $\ldots$ & $\ldots$ & 0.182 & $\ldots$ \\
\hline NGC $4736 \mathrm{P}$ & 0.133 & 0.036 & -0.008 & 0.126 & 0.120 & 0.013 \\
\hline NGC 4736 D & 0.015 & 0.112 & 0.009 & 0.025 & 0.107 & 0.020 \\
\hline NGC 4736 Enuc. $1 \mathrm{D}$ & 0.015 & 0.015 & 0.030 & 0.096 & 0.044 & 0.109 \\
\hline NGC 4736 Enuc. $1 \mathrm{~A}$ & 0.049 & 0.099 & 0.093 & 0.032 & 0.093 & 0.106 \\
\hline NGC 4736 Enuc. 1 B & 0.148 & 0.038 & 0.065 & 0.053 & 0.024 & 0.063 \\
\hline NGC 4736 Enuc. $1 \mathrm{C}$ & -0.002 & 0.062 & 0.027 & 0.001 & 0.054 & 0.043 \\
\hline NGC 4736 Enuc. $1 \mathrm{E}$ & 0.013 & $\ldots$ & 0.015 & 0.148 & $\ldots$ & 0.110 \\
\hline NGC $4826 \mathrm{C}$ & 0.049 & 0.086 & 0.033 & 0.068 & 0.177 & 0.152 \\
\hline NGC $4826 \mathrm{~B}$ & 0.134 & 0.109 & 0.039 & 0.185 & 0.205 & 0.155 \\
\hline NGC 4826 A & 0.779 & 0.470 & 0.174 & 0.064 & 0.103 & 0.062 \\
\hline NGC $4826 \mathrm{~F}^{\mathrm{b}}$ & 0.236 & 0.899 & 0.044 & 0.021 & 0.093 & 0.012 \\
\hline NGC $4826 \mathrm{E}$ & 0.048 & 0.087 & 0.019 & 0.110 & 0.189 & 0.136 \\
\hline NGC 4826 D & 0.077 & 0.075 & 0.030 & 0.136 & 0.229 & 0.096 \\
\hline
\end{tabular}


Table A2

(Continued)

\begin{tabular}{|c|c|c|c|c|c|c|}
\hline Source ID & $\begin{array}{l}\text { Back } 3 \mathrm{GHz} \\
(\mathrm{mJy})\end{array}$ & $\begin{array}{l}\text { Back } 15 \mathrm{GHz} \\
(\mathrm{mJy})\end{array}$ & $\begin{array}{l}\text { Back } 33 \mathrm{GHz} \\
(\mathrm{mJy})\end{array}$ & $f_{\mathrm{Back}}^{3 \mathrm{GHz} \text { a }}$ & $f_{\mathrm{Back}}^{15 \mathrm{GHz} \text { a }}$ & $f_{\text {Back }}^{33 \mathrm{GHz} \text { a }}$ \\
\hline NGC $5055 \mathrm{C}$ & 0.005 & $\ldots$ & $\ldots$ & 0.033 & $\ldots$ & $\ldots$ \\
\hline NGC 5055 D & 0.023 & 0.040 & $\ldots$ & 0.106 & 0.207 & $\ldots$ \\
\hline NGC 5055 A & 0.204 & 0.150 & 0.066 & 0.103 & 0.149 & 0.197 \\
\hline NGC $5055 \mathrm{~B}$ & -0.011 & 0.002 & $\ldots$ & 0.010 & 0.006 & $\ldots$ \\
\hline NGC 5055 Enuc. 1 & 0.002 & 0.024 & 0.020 & 0.005 & 0.055 & 0.050 \\
\hline NGC 5194 Enuc. 6 & 0.034 & 0.043 & 0.044 & 0.050 & 0.082 & 0.078 \\
\hline NGC 5194 Enuc. 3 B & $\cdots$ & 0.026 & 0.022 & $\cdots$ & 0.074 & 0.165 \\
\hline NGC 5194 Enuc. 3 A & 0.050 & 0.035 & 0.040 & 0.062 & 0.064 & 0.069 \\
\hline NGC 5194 Enuc. 11 G & -0.031 & $\ldots$ & 0.009 & 0.105 & $\ldots$ & 0.030 \\
\hline NGC 5194 Enuc. 11 A & 0.053 & $\ldots$ & 0.008 & 0.114 & $\ldots$ & 0.036 \\
\hline NGC 5194 Enuc. 11 B & $\ldots$ & 0.035 & 0.010 & $\ldots$ & 0.220 & 0.053 \\
\hline NGC 5194 Enuc. $11 \mathrm{C}$ & $\cdots$ & 0.004 & 0.009 & $\cdots$ & 0.042 & 0.064 \\
\hline NGC 5194 Enuc. 11 D & 0.074 & 0.029 & 0.000 & 0.087 & 0.109 & 0.004 \\
\hline NGC $5194 \mathrm{H}$ & 0.055 & -0.011 & -0.017 & 0.019 & 0.009 & 0.060 \\
\hline NGC 5194L & 0.333 & 0.092 & 0.031 & 0.200 & 0.140 & 0.179 \\
\hline NGC 5194 M & 0.089 & 0.050 & 0.012 & 0.130 & 0.199 & 0.090 \\
\hline NGC 5194 Enuc. $11 \mathrm{E}$ & 0.018 & 0.034 & 0.009 & 0.086 & 0.149 & 0.056 \\
\hline NGC 5194 A & 0.240 & 0.079 & 0.008 & 0.170 & 0.144 & 0.030 \\
\hline NGC 5194 Enuc. $11 \mathrm{~F}$ & 0.025 & 0.015 & 0.005 & 0.100 & 0.101 & 0.036 \\
\hline NGC 5194 K & 0.095 & 0.108 & 0.011 & 0.111 & 0.165 & 0.048 \\
\hline NGC $5194 \mathrm{~B}$ & 0.028 & 0.018 & 0.008 & 0.014 & 0.029 & 0.022 \\
\hline NGC 5194 G & 0.220 & 0.095 & 0.010 & 0.194 & 0.198 & 0.100 \\
\hline NGC $5194 \mathrm{~J}$ & 0.077 & 0.036 & 0.015 & 0.130 & 0.261 & 0.200 \\
\hline NGC 5194 E & 0.559 & 0.306 & 0.094 & 0.045 & 0.070 & 0.056 \\
\hline NGC $5194 \mathrm{O}$ & 0.011 & 0.111 & $\ldots$ & 0.028 & 0.238 & $\ldots$ \\
\hline NGC 5194 I & 0.474 & 0.124 & 0.039 & 0.088 & 0.087 & 0.055 \\
\hline NGC $5194 \mathrm{C}^{\mathrm{b}}$ & 0.491 & 0.203 & 0.050 & 0.046 & 0.072 & 0.041 \\
\hline NGC 5194 Enuc. $1 \mathrm{~A}$ & 0.025 & 0.001 & 0.007 & 0.076 & 0.010 & 0.027 \\
\hline NGC 5194 Enuc. 4 D & $\ldots$ & $\ldots$ & 0.007 & $\ldots$ & $\ldots$ & 0.057 \\
\hline NGC 5194N & 0.237 & 0.069 & $\ldots$ & 0.179 & 0.258 & $\ldots$ \\
\hline NGC $5194 \mathrm{~F}$ & 0.024 & 0.041 & 0.014 & 0.032 & 0.079 & 0.044 \\
\hline NGC 5194 Enuc. $4 \mathrm{C}$ & 0.021 & 0.004 & 0.004 & 0.129 & 0.036 & 0.033 \\
\hline NGC $5194 \mathrm{P}$ & 0.112 & 0.014 & 0.002 & 0.157 & 0.048 & 0.010 \\
\hline NGC 5194 Enuc. 4 E & $\ldots$ & $\ldots$ & 0.002 & $\ldots$ & $\ldots$ & 0.034 \\
\hline NGC 5194 Enuc. 10 & 0.065 & 0.041 & 0.037 & 0.092 & 0.117 & 0.105 \\
\hline NGC 5194 Enuc. 4 B & 0.005 & $\cdots$ & -0.002 & 0.046 & $\cdots$ & 0.026 \\
\hline NGC 5194 Enuc. 5 B & 0.021 & 0.005 & 0.000 & 0.079 & 0.027 & 0.001 \\
\hline NGC 5194 Enuc. $4 \mathrm{~F}$ & 0.018 & 0.004 & 0.000 & 0.083 & 0.027 & 0.003 \\
\hline NGC 5194 Enuc. 4 G & 0.029 & 0.026 & -0.000 & 0.052 & 0.090 & 0.004 \\
\hline NGC 5194 Enuc. 5 A & 0.024 & 0.022 & 0.014 & 0.038 & 0.063 & 0.038 \\
\hline NGC 5194 Enuc. 9 & 0.002 & -0.020 & 0.010 & 0.004 & 0.055 & 0.026 \\
\hline NGC 5194 Enuc. 8 C & 0.005 & 0.032 & $\ldots$ & 0.014 & 0.118 & $\ldots$ \\
\hline NGC 5194 Enuc. 8 B & 0.052 & 0.048 & 0.005 & 0.078 & 0.075 & 0.024 \\
\hline NGC 5194 Enuc. 7 E & $\ldots$ & 0.028 & 0.002 & $\ldots$ & 0.150 & 0.008 \\
\hline NGC 5194 Enuc. 8 A & 0.070 & 0.044 & -0.012 & 0.126 & 0.082 & 0.030 \\
\hline NGC 5194 Enuc. 7 A & 0.103 & 0.022 & 0.019 & 0.086 & 0.055 & 0.057 \\
\hline NGC 5194 Enuc. 7 C & 0.048 & 0.009 & -0.003 & 0.117 & 0.049 & 0.018 \\
\hline NGC 5194 Enuc. 7 B & 0.022 & -0.013 & $\cdots$ & 0.067 & 0.081 & $\ldots$ \\
\hline NGC 5194 Enuc. 7 F & 0.011 & 0.052 & $\ldots$ & 0.002 & 0.029 & $\cdots$ \\
\hline NGC 5194 Enuc. 8 D & 0.002 & -0.018 & $\ldots$ & 0.006 & 0.092 & $\ldots$ \\
\hline NGC 5398 & 0.000 & 0.024 & 0.002 & 0.000 & 0.014 & 0.001 \\
\hline NGC 5457 Enuc. $6 \mathrm{~A}$ & 0.092 & 0.099 & 0.018 & 0.088 & 0.106 & 0.033 \\
\hline NGC 5457 Enuc. $6 \mathrm{E}$ & 0.071 & 0.077 & 0.024 & 0.106 & 0.174 & 0.052 \\
\hline NGC 5457 Enuc. $6 \mathrm{~B}$ & 0.183 & 0.118 & 0.060 & 0.078 & 0.075 & 0.079 \\
\hline NGC 5457 Enuc. $6 \mathrm{C}$ & 0.060 & 0.066 & 0.013 & 0.060 & 0.090 & 0.023 \\
\hline NGC 5457 Enuc. $6 \mathrm{D}$ & 0.023 & 0.031 & 0.005 & 0.040 & 0.084 & 0.016 \\
\hline NGC 5457 Enuc. $6 \mathrm{~F}$ & 0.009 & 0.009 & $\ldots$ & 0.042 & 0.084 & $\ldots$ \\
\hline NGC 5457 Enuc. 2 B & 0.001 & -0.001 & $\ldots$ & 0.007 & 0.012 & $\ldots$ \\
\hline NGC 5457 Enuc. 2 C & -0.007 & 0.004 & $\ldots$ & 0.046 & 0.077 & $\ldots$ \\
\hline NGC 5457 Enuc. 5 B & 0.099 & 0.118 & 0.033 & 0.067 & 0.099 & 0.029 \\
\hline NGC 5457 Enuc. $5 \mathrm{~A}^{\mathrm{b}}$ & 0.101 & 0.115 & 0.034 & 0.048 & 0.053 & 0.027 \\
\hline NGC 5457 Enuc. 5 C & 0.103 & 0.062 & 0.045 & 0.118 & 0.100 & 0.065 \\
\hline NGC 5457 Enuc. 1 & 0.009 & $\ldots$ & 0.005 & 0.083 & $\ldots$ & 0.058 \\
\hline
\end{tabular}


Table A2

(Continued)

\begin{tabular}{|c|c|c|c|c|c|c|}
\hline Source ID & $\begin{array}{l}\text { Back } 3 \mathrm{GHz} \\
(\mathrm{mJy})\end{array}$ & $\begin{array}{c}\text { Back } 15 \mathrm{GHz} \\
(\mathrm{mJy})\end{array}$ & $\begin{array}{l}\text { Back } 33 \mathrm{GHz} \\
(\mathrm{mJy})\end{array}$ & $f_{\text {Back }}^{3 \mathrm{GHz} \text { a }}$ & $f_{\mathrm{Back}}^{15 \mathrm{GHz} \text { a }}$ & $f_{\mathrm{Back}}^{33 \mathrm{GHz}}$ \\
\hline NGC 5457 & 0.015 & 0.021 & 0.013 & 0.017 & 0.086 & 0.035 \\
\hline NGC 5457 Enuc. $3 \mathrm{E}$ & -0.008 & 0.003 & $\cdots$ & 0.034 & 0.016 & $\cdots$ \\
\hline NGC 5457 Enuc. 3 D & 0.013 & 0.032 & $\ldots$ & 0.022 & 0.090 & $\ldots$ \\
\hline NGC 5457 Enuc. 3 A & 0.029 & 0.065 & 0.043 & 0.032 & 0.075 & 0.065 \\
\hline NGC 5457 Enuc. 3 B & 0.257 & 0.299 & 0.087 & 0.022 & 0.032 & 0.012 \\
\hline NGC 5457 Enuc. $3 \mathrm{C}$ & 0.030 & 0.018 & -0.001 & 0.031 & 0.033 & 0.003 \\
\hline NGC 5457 Enuc. 4 A & 0.043 & 0.026 & -0.001 & 0.104 & 0.101 & 0.006 \\
\hline NGC 5457 Enuc. $4 \mathrm{~B}$ & 0.049 & 0.045 & 0.010 & 0.145 & 0.160 & 0.038 \\
\hline NGC 5457 Enuc. $4 \mathrm{C}$ & 0.081 & 0.078 & 0.041 & 0.098 & 0.140 & 0.090 \\
\hline NGC 5457 Enuc. $4 \mathrm{D}$ & 0.081 & 0.047 & 0.026 & 0.080 & 0.081 & 0.073 \\
\hline NGC 5457 Enuc. $7 \mathrm{D}^{\mathrm{b}}$ & 0.238 & 0.130 & 0.074 & 0.040 & 0.030 & 0.019 \\
\hline NGC 5457 Enuc. 7 B & 0.115 & 0.151 & 0.081 & 0.054 & 0.200 & 0.111 \\
\hline NGC $5713 \mathrm{~A}$ & 0.095 & 0.101 & 0.035 & 0.052 & 0.106 & 0.058 \\
\hline NGC $5713 \mathrm{~F}$ & 0.035 & 0.063 & 0.025 & 0.094 & 0.194 & 0.127 \\
\hline NGC 5713 B & 0.234 & 0.128 & 0.054 & 0.062 & 0.075 & 0.041 \\
\hline NGC 5713 Enuc. 2 & 0.100 & 0.081 & -0.013 & 0.081 & 0.105 & 0.046 \\
\hline NGC 5713 Enuc. 2 A & 0.185 & 0.141 & 0.024 & 0.096 & 0.131 & 0.038 \\
\hline NGC 5713 C & 0.420 & 0.186 & 0.103 & 0.078 & 0.093 & 0.069 \\
\hline NGC $5713 \mathrm{G}^{\mathrm{b}}$ & 1.348 & 1.190 & 0.271 & 0.120 & 0.176 & 0.126 \\
\hline NGC 5713 D & 0.334 & 0.182 & 0.096 & 0.044 & 0.056 & 0.040 \\
\hline NGC 5713 Enuc. 1 A & $\ldots$ & 0.031 & $\ldots$ & $\ldots$ & 0.164 & $\ldots$ \\
\hline NGC 5713 E & 0.209 & 0.142 & 0.016 & 0.151 & 0.165 & 0.028 \\
\hline NGC 5866 & 0.088 & 0.105 & 0.035 & 0.013 & 0.021 & 0.016 \\
\hline NGC 6946 Enuc. 4 A & 0.047 & 0.035 & 0.045 & 0.122 & 0.103 & 0.116 \\
\hline NGC 6946 Enuc. 4 B & 0.004 & 0.011 & 0.023 & 0.020 & 0.052 & 0.112 \\
\hline NGC 6946 Enuc. $4 \mathrm{H}$ & -0.002 & -0.003 & 0.000 & 0.023 & 0.028 & 0.002 \\
\hline NGC 6946 Enuc. 8 A & 0.223 & 0.041 & 0.042 & 0.099 & 0.056 & 0.051 \\
\hline NGC 6946 Enuc. 5 A & $\ldots$ & $\cdots$ & 0.007 & $\ldots$ & $\ldots$ & 0.064 \\
\hline NGC 6946 Enuc. 8 B & $\ldots$ & 0.015 & $\ldots$ & $\ldots$ & 0.067 & $\ldots$ \\
\hline NGC 6946 Enuc. 5 B & 0.009 & 0.027 & 0.010 & 0.028 & 0.071 & 0.026 \\
\hline NGC 6946 Enuc. 3 A & -0.014 & 0.014 & 0.010 & 0.135 & 0.069 & 0.043 \\
\hline NGC 6946 Enuc. 3 B & -0.011 & 0.029 & 0.014 & 0.089 & 0.143 & 0.069 \\
\hline NGC 6946 A & 0.751 & 0.529 & 0.154 & 0.036 & 0.062 & 0.035 \\
\hline NGC 6946 Enuc. 3 C & 0.015 & 0.049 & 0.028 & 0.031 & 0.103 & 0.065 \\
\hline NGC 6946 B & 0.007 & 0.027 & -0.007 & 0.015 & 0.069 & 0.025 \\
\hline NGC 6946 C & 0.101 & 0.060 & 0.034 & 0.136 & 0.096 & 0.107 \\
\hline NGC 6946 Enuc. $6 \mathrm{~J}$ & 0.017 & 0.000 & 0.014 & 0.148 & 0.005 & 0.138 \\
\hline NGC 6946 Enuc. 6 I & 0.025 & 0.008 & 0.022 & 0.113 & 0.073 & 0.210 \\
\hline NGC 6946 Enuc. $6 \mathrm{H}$ & 0.019 & 0.004 & 0.014 & 0.101 & 0.034 & 0.089 \\
\hline NGC 6946 Enuc. 6 F & 0.013 & 0.012 & 0.015 & 0.056 & 0.188 & 0.127 \\
\hline NGC 6946 Enuc. 6 E & $\ldots$ & 0.008 & 0.008 & $\ldots$ & 0.050 & 0.049 \\
\hline NGC 6946 Enuc. 6 B & 0.011 & 0.011 & 0.001 & 0.032 & 0.038 & 0.007 \\
\hline NGC 6946 Enuc. 6 C & 0.006 & 0.006 & $\ldots$ & 0.056 & 0.100 & $\ldots$ \\
\hline NGC 6946 Enuc. $9 \mathrm{E}$ & -0.004 & 0.006 & $\ldots$ & 0.031 & 0.077 & $\ldots$ \\
\hline NGC 6946 Enuc. 9 D & 0.031 & 0.023 & $\ldots$ & 0.161 & 0.104 & $\ldots$ \\
\hline NGC 6946 Enuc. 9 A & 0.060 & 0.090 & 0.033 & 0.070 & 0.122 & 0.053 \\
\hline NGC 6946 Enuc. 6 L & 0.051 & 0.006 & 0.010 & 0.072 & 0.026 & 0.026 \\
\hline NGC 6946 Enuc. 9 C & -0.007 & 0.020 & 0.011 & 0.057 & 0.100 & 0.102 \\
\hline NGC 6946 Enuc. 9 B & 0.009 & 0.024 & 0.015 & 0.057 & 0.143 & 0.117 \\
\hline NGC 6946 Enuc. 7 A & -0.007 & 0.045 & 0.013 & 0.035 & 0.060 & 0.027 \\
\hline NGC 6946 Enuc. 7 B & -0.008 & 0.017 & 0.009 & 0.059 & 0.079 & 0.057 \\
\hline NGC 6946 Enuc. 1 A & -0.003 & 0.029 & 0.031 & 0.004 & 0.045 & 0.047 \\
\hline NGC 6946 Enuc. 2 C & 0.003 & 0.004 & $\ldots$ & 0.018 & 0.025 & $\ldots$ \\
\hline NGC $7331 \mathrm{G}$ & 0.120 & $\ldots$ & $\ldots$ & 0.172 & $\ldots$ & $\ldots$ \\
\hline NGC $7331 \mathrm{~F}$ & 0.073 & 0.115 & $\ldots$ & 0.106 & 0.268 & $\ldots$ \\
\hline NGC $7331 \mathrm{H}$ & 0.069 & -0.029 & $\ldots$ & 0.052 & 0.025 & $\ldots$ \\
\hline NGC 7331 I & 0.076 & 0.143 & $\cdots$ & 0.062 & 0.097 & $\cdots$ \\
\hline NGC $7331 \mathrm{~A}$ & -0.020 & 0.045 & $\ldots$ & 0.123 & 0.123 & $\ldots$ \\
\hline NGC $7331 \mathrm{E}$ & 0.061 & $\ldots$ & -0.057 & 0.067 & $\ldots$ & 0.117 \\
\hline NGC 7331 B & 0.055 & 0.111 & $\ldots$ & 0.108 & 0.138 & $\ldots$ \\
\hline NGC $7331 \mathrm{D}$ & 0.014 & 0.076 & $\ldots$ & 0.096 & 0.170 & $\ldots$ \\
\hline NGC 7793 Enuc. $1 \mathrm{E}$ & -0.001 & -0.004 & $\ldots$ & 0.008 & 0.059 & $\ldots$ \\
\hline NGC 7793 Enuc. 3 & -0.006 & 0.002 & $\ldots$ & 0.030 & 0.009 & $\ldots$ \\
\hline
\end{tabular}


Table A2

(Continued)

\begin{tabular}{|c|c|c|c|c|c|c|}
\hline Source ID & $\begin{array}{c}\text { Back } 3 \mathrm{GHz} \\
(\mathrm{mJy})\end{array}$ & $\underset{(\mathrm{mJy})}{\text { Back } 15 \mathrm{GHz}}$ & $\begin{array}{c}\text { Back } 33 \mathrm{GHz} \\
(\mathrm{mJy})\end{array}$ & $f_{\text {Back }}^{3 \mathrm{GHz} \text { a }}$ & $f_{\text {Back }}^{15 \mathrm{GHz} \text { a }}$ & $f_{\text {Back }}^{33 \mathrm{GHz} \text { a }}$ \\
\hline NGC 7793 Enuc. $1 \mathrm{~A}$ & 0.006 & 0.004 & $\cdots$ & 0.059 & 0.051 & $\cdots$ \\
\hline NGC 7793 C & -0.004 & 0.004 & -0.001 & 0.020 & 0.025 & 0.007 \\
\hline NGC 7793 A & -0.008 & 0.013 & -0.003 & 0.035 & 0.067 & 0.016 \\
\hline NGC 7793 Enuc. 1 B & $\cdots$ & 0.008 & $\cdots$ & $\cdots$ & 0.079 & $\ldots$ \\
\hline NGC 7793 Enuc. $1 \mathrm{C}$ & -0.005 & 0.001 & $\cdots$ & 0.026 & 0.005 & $\cdots$ \\
\hline NGC 7793 Enuc. 2 & $\cdots$ & $\cdots$ & -0.006 & $\cdots$ & $\cdots$ & 0.042 \\
\hline \multicolumn{7}{|c|}{ Likely Background Galaxies } \\
\hline NGC $0925 \mathrm{C}$ & -0.001 & -0.004 & 0.003 & 0.001 & 0.006 & 0.011 \\
\hline IC $342 \mathrm{E}$ & 0.113 & 0.039 & $\cdots$ & 0.143 & 0.139 & $\cdots$ \\
\hline Holmberg II C & 0.006 & 0.011 & 0.019 & 0.041 & 0.080 & 0.115 \\
\hline NGC 2841 B & -0.003 & $\ldots$ & $\cdots$ & 0.026 & $\cdots$ & $\cdots$ \\
\hline NGC 2976 Enuc. $1 \mathrm{E}$ & -0.005 & $\cdots$ & $\cdots$ & 0.031 & $\cdots$ & $\cdots$ \\
\hline NGC 3077 B & -0.004 & 0.009 & $\cdots$ & 0.066 & 0.078 & $\cdots$ \\
\hline NGC 3190 B & 0.089 & 0.027 & 0.017 & 0.042 & 0.040 & 0.034 \\
\hline NGC 3265 B & 0.001 & -0.001 & $\cdots$ & 0.001 & 0.017 & $\cdots$ \\
\hline NGC $3351 \mathrm{D}$ & -0.011 & 0.014 & $\cdots$ & 0.035 & 0.139 & $\cdots$ \\
\hline NGC 3627 Enuc. 1 B & -0.000 & $\ldots$ & $\ldots$ & 0.003 & $\ldots$ & $\ldots$ \\
\hline NGC $4569 \mathrm{~B}$ & -0.017 & $\ldots$ & $\ldots$ & 0.034 & $\ldots$ & $\ldots$ \\
\hline NGC $4625 \mathrm{~B}$ & 0.001 & 0.004 & 0.001 & 0.001 & 0.016 & 0.004 \\
\hline NGC 6946 Enuc. $4 \mathrm{~F}$ & 0.145 & 0.105 & 0.032 & 0.025 & 0.061 & 0.035 \\
\hline NGC 6946 Enuc. 4 G & -0.044 & -0.002 & 0.011 & 0.018 & 0.002 & 0.022 \\
\hline \multicolumn{7}{|c|}{ Likely Associated with Supernovae } \\
\hline NGC 2403 Enuc. 3 D & $\cdots$ & -0.038 & $\cdots$ & $\ldots$ & 0.022 & $\cdots$ \\
\hline NGC 4736 B & -0.016 & $\ldots$ & $\ldots$ & 0.100 & $\ldots$ & $\cdots$ \\
\hline NGC $4736 \mathrm{C}$ & -0.027 & $\cdots$ & $\cdots$ & 0.169 & $\ldots$ & $\cdots$ \\
\hline NGC $5194 \mathrm{D}$ & 0.034 & 0.065 & 0.045 & 0.028 & 0.103 & 0.055 \\
\hline NGC 5194 Enuc. 7 D & 0.028 & -0.003 & $\cdots$ & 0.018 & 0.017 & $\cdots$ \\
\hline NGC $6946 \mathrm{~F}$ & 0.016 & 0.012 & $\cdots$ & 0.079 & 0.108 & $\ldots$ \\
\hline NGC 6946 Enuc. 6 D & 0.004 & 0.013 & -0.003 & 0.013 & 0.036 & 0.015 \\
\hline NGC 6946 Enuc. 2 B & 0.006 & 0.087 & 0.029 & 0.005 & 0.061 & 0.015 \\
\hline NGC $7331 \mathrm{C}$ & 0.040 & 0.379 & 0.055 & 0.051 & 0.205 & 0.076 \\
\hline NGC 7793 B & -0.002 & $\cdots$ & $\cdots$ & 0.013 & $\cdots$ & $\cdots$ \\
\hline \multicolumn{7}{|c|}{ Likely AME Candidates } \\
\hline NGC 2403 Enuc. 5 D & 0.045 & 0.073 & 0.036 & 0.071 & 0.115 & 0.059 \\
\hline NGC 2403 Enuc. 2 E & 0.038 & 0.057 & 0.022 & 0.032 & 0.059 & 0.018 \\
\hline NGC 2403 Enuc. 3 A & 0.015 & 0.030 & 0.033 & 0.038 & 0.075 & 0.063 \\
\hline NGC 2403 Enuc. 3 C & 0.112 & 0.070 & 0.093 & 0.045 & 0.037 & 0.049 \\
\hline NGC 3627 Enuc. 1 D & 0.014 & 0.048 & 0.014 & 0.036 & 0.086 & 0.023 \\
\hline NGC 3627 Enuc. $1 \mathrm{C}$ & 0.005 & 0.085 & -0.004 & 0.011 & 0.134 & 0.004 \\
\hline NGC 4254 Enuc. 2 A & 0.005 & 0.008 & 0.011 & 0.041 & 0.052 & 0.049 \\
\hline NGC 4254 Enuc. $1 \mathrm{~A}$ & 0.013 & 0.007 & 0.020 & 0.037 & 0.053 & 0.088 \\
\hline NGC $4631 \mathrm{C}$ & 0.115 & 0.020 & 0.081 & 0.066 & 0.023 & 0.065 \\
\hline NGC $4631 \mathrm{D}$ & 0.065 & 0.013 & 0.078 & 0.042 & 0.025 & 0.083 \\
\hline NGC $4631 \mathrm{E}$ & 0.075 & 0.038 & 0.065 & 0.080 & 0.083 & 0.083 \\
\hline NGC $4631 \mathrm{~F}$ & 0.418 & 0.259 & 0.212 & 0.061 & 0.079 & 0.053 \\
\hline NGC 4631 Enuc. 2 A & 0.033 & 0.006 & 0.005 & 0.055 & 0.027 & 0.013 \\
\hline NGC 4725 B & -0.003 & 0.001 & 0.003 & 0.024 & 0.005 & 0.008 \\
\hline NGC 5194 Enuc. 2 & 0.019 & 0.011 & 0.018 & 0.015 & 0.024 & 0.032 \\
\hline NGC 5194 Enuc. $1 \mathrm{C}$ & 0.053 & 0.030 & 0.033 & 0.098 & 0.064 & 0.045 \\
\hline NGC 5194 Enuc. 1 B & 0.034 & 0.025 & 0.023 & 0.069 & 0.051 & 0.043 \\
\hline NGC 5194 Enuc. 4 A & 0.040 & -0.004 & 0.018 & 0.056 & 0.012 & 0.044 \\
\hline NGC 5457 Enuc. 2 A & 0.020 & 0.007 & 0.006 & 0.050 & 0.026 & 0.017 \\
\hline NGC 5457 Enuc. 7 A & 0.084 & 0.071 & 0.048 & 0.079 & 0.127 & 0.068 \\
\hline NGC 5457 Enuc. 7 C & 0.263 & 0.243 & 0.162 & 0.074 & 0.135 & 0.084 \\
\hline NGC 6946 Enuc. 4 C & 0.026 & 0.037 & 0.042 & 0.017 & 0.026 & 0.027 \\
\hline NGC 6946 Enuc. 4 D & -0.001 & 0.013 & 0.004 & 0.011 & 0.016 & 0.005 \\
\hline NGC 6946 Enuc. $4 \mathrm{E}$ & 0.014 & 0.035 & 0.023 & 0.011 & 0.026 & 0.018 \\
\hline NGC 6946 E & 0.060 & 0.062 & 0.055 & 0.083 & 0.086 & 0.043 \\
\hline NGC 6946 D & 0.018 & 0.064 & 0.005 & 0.045 & 0.126 & 0.010 \\
\hline
\end{tabular}


Table A2

(Continued)

\begin{tabular}{|c|c|c|c|c|c|c|}
\hline Source ID & $\begin{array}{c}\text { Back } 3 \mathrm{GHz} \\
(\mathrm{mJy})\end{array}$ & $\begin{array}{c}\text { Back } 15 \mathrm{GHz} \\
(\mathrm{mJy})\end{array}$ & $\begin{array}{c}\text { Back } 33 \mathrm{GHz} \\
(\mathrm{mJy})\end{array}$ & $f_{\mathrm{Back}}^{3 \mathrm{GHz}}$ & $f_{\mathrm{Back}}^{15 \mathrm{GHz} \text { a }}$ & $f_{\mathrm{Back}}^{33 \mathrm{GHz} \text { a }}$ \\
\hline NGC 6946 Enuc. $6 \mathrm{M}$ & -0.005 & -0.012 & 0.006 & 0.009 & 0.133 & 0.014 \\
\hline NGC 6946 Enuc. 6 G & 0.050 & 0.019 & 0.044 & 0.064 & 0.038 & 0.080 \\
\hline NGC 6946 Enuc. $6 \mathrm{~K}$ & 0.063 & 0.025 & 0.051 & 0.090 & 0.064 & 0.112 \\
\hline NGC 6946 Enuc. $6 \mathrm{~A}^{\mathrm{b}}$ & 0.080 & 0.036 & 0.097 & 0.043 & 0.033 & 0.081 \\
\hline NGC 6946 Enuc. 1 B & 0.078 & 0.006 & 0.009 & 0.108 & 0.016 & 0.013 \\
\hline NGC 6946 Enuc. 2 A & -0.008 & 0.028 & 0.004 & 0.074 & 0.092 & 0.012 \\
\hline NGC 7793 Enuc. 1 D & -0.010 & 0.001 & $\cdots$ & 0.044 & 0.006 & $\cdots$ \\
\hline
\end{tabular}

Notes.

a The fractional contribution of the absolute value of the local background measurement to the aperture flux density.

$\mathrm{b}$ The aperture used for this star-forming region contained multiple smaller individual regions.

\section{ORCID iDs}

S. T. Linden (1) https://orcid.org/0000-0002-1000-6081

E. J. Murphy (i) https://orcid.org/0000-0001-7089-7325

E. Momjian (1) https://orcid.org/0000-0003-3168-5922

D. S. Meier (ib https://orcid.org/0000-0001-9436-9471

E. Schinnerer (ii) https://orcid.org/0000-0002-3933-7677

J. L. Turner (i) https://orcid.org/0000-0003-4625-2951

\section{References}

Aniano, G., Draine, B. T., Gordon, K. D., \& Sandstrom, K. 2011, PASP, 123,1218

Bell, E. F. 2003, ApJ, 586, 794

Bot, C., Ysard, N., Paradis, D., et al. 2010, A\&A, 523, A20

Ciardullo, R., Feldmeier, J. J., Jacoby, G. H., et al. 2002, ApJ, 577, 31

Clemens, M. S., Scaife, A., Vega, O., \& Bressan, A. 2010, MNRAS, 405, 887

Clemens, M. S., Vega, O., Bressan, A., et al. 2008, A\&A, 477, 95

Condon, J. J. 1992, ARA\&A, 30, 575

Condon, J. J., Cotton, W. D., Fomalont, E. B., et al. 2012, ApJ, 758, 23

Condon, J. J., \& Yin, Q. F. 1990, ApJ, 357, 97

Conway, J. E., Cornwell, T. J., \& Wilkinson, P. N. 1990, MNRAS, 246, 490

Cornwell, T. J. 2008, ISTSP, 2, 793

Dale, D. A., Cohen, S. A., Johnson, L. C., et al. 2009, ApJ, 703, 517

Dale, D. A., Gil de Paz, A., Gordon, K. D., et al. 2007, ApJ, 655, 863

de Jong, T., Klein, U., Wielebinski, R., \& Wunderlich, E. 1985, A\&A, 147, L6

de Vaucouleurs, G., de Vaucouleurs, A., Corwin, Herold, G. J., et al. 1991, Third Reference Catalogue of Bright Galaxies (Vol I, II, and III) (New York: Springer)

Dickinson, C., Ali-Haïmoud, Y., Barr, A., et al. 2018, NewAR, 80, 1

Draine, B. T. 2003, ARA\&A, 41, 241

Draine, B. T., \& Lazarian, A. 1998, ApJ, 508, 157

Engelbracht, C. W., Rieke, G. H., Gordon, K. D., et al. 2008, ApJ, 678, 804 Erickson, W. C. 1957, ApJ, 126, 480

Foreman-Mackey, D., Hogg, D. W., Lang, D., \& Goodman, J. 2013, PASP, 125,306

Freedman, W. L., Madore, B. F., Gibson, B. K., et al. 2001, ApJ, 553, 47

Green, D. A. 2011, BASI, 39, 289

Guillochon, J., Parrent, J., Kelley, L. Z., \& Margutti, R. 2017, ApJ, 835, 64

Heesen, V., Brinks, E., Leroy, A. K., et al. 2014, AJ, 147, 103

Helou, G., Soifer, B. T., \& Rowan-Robinson, M. 1985, ApJL, 298, L7

Hensley, B., Murphy, E., \& Staguhn, J. 2015, MNRAS, 449, 809
Hogg, D. W., Bovy, J., \& Lang, D. 2010, arXiv:1008.4686

Hughes, A., Staveley-Smith, L., Kim, S., Wolleben, M., \& Filipović, M. 2007, MNRAS, 382, 543

Jarrett, T. H., Chester, T., Cutri, R., Schneider, S. E., \& Huchra, J. P. 2003, AJ, 125,525

Kennicutt, R. C., Calzetti, D., Aniano, G., et al. 2011, PASP, 123, 1347

Kennicutt, R. C., Jr., Armus, L., Bendo, G., et al. 2003, PASP, 115, 928

Klein, U., Wielebinski, R., \& Beck, R. 1984, A\&A, 135, 213

Klein, U., Wielebinski, R., \& Morsi, H. W. 1988, A\&A, 190, 41

Koyama, K., Petre, R., Gotthelf, E. V., et al. 1995, Natur, 378, 255

Lacki, B. C., \& Thompson, T. A. 2010, ApJ, 717, 196

Lacki, B. C., Thompson, T. A., \& Quataert, E. 2010, ApJ, 717, 1

Leitherer, C., Schaerer, D., Goldader, J. D., et al. 1999, ApJS, 123, 3

Leroy, A. K., Bigiel, F., de Blok, W. J. G., et al. 2012, AJ, 144, 3

Leroy, A. K., Evans, A. S., Momjian, E., et al. 2011, ApJL, 739, L25

Linden, S. T., Song, Y., Evans, A. S., et al. 2019, ApJ, 881, 70

Lonsdale Persson, C. J., \& Helou, G. 1987, ApJ, 314, 513

McMullin, J. P., Waters, B., Schiebel, D., Young, W., \& Golap, K. 2007, in ASP Conf. Ser. 376, Astronomical Data Analysis Software and Systems XVI, ed. R. A. Shaw, F. Hill, \& D. J. Bell (San Francisco, CA: ASP), 127

Meier, D. S., Turner, J. L., \& Beck, S. C. 2002, AJ, 124, 877

Moustakas, J., Kennicutt, R. C., Jr., Tremonti, C. A., et al. 2010, ApJS, 190,233

Murphy, E. J., Braun, R., Helou, G., et al. 2006, ApJ, 638, 157

Murphy, E. J., Bremseth, J., Mason, B. S., et al. 2012, ApJ, 761, 97

Murphy, E. J., Condon, J. J., Schinnerer, E., et al. 2011, ApJ, 737, 67

Murphy, E. J., Dong, D., Momjian, E., et al. 2018a, ApJS, 234, 24

Murphy, E. J., Helou, G., Condon, J. J., et al. 2010, ApJL, 709, L108

Murphy, E. J., Linden, S. T., Dong, D., et al. 2018b, ApJ, 862, 20

Murphy, E. J., Stierwalt, S., Armus, L., Condon, J. J., \& Evans, A. S. 2013 , ApJ, 768, 2

Niklas, S., \& Beck, R. 1997, A\&A, 320, 54

Peel, M. W., Dickinson, C., Davies, R. D., Clements, D. L., \& Beswick, R. J. 2011, MNRAS, 416, L99

Perley, R. A., \& Butler, B. J. 2013, ApJS, 204, 19

Rau, U., \& Cornwell, T. J. 2011, A\&A, 532, A71

Sault, R. J., \& Wieringa, M. H. 1994, A\&AS, 108, 585

Schlegel, D. J., Finkbeiner, D. P., \& Davis, M. 1998, ApJ, 500, 525

Seibert, M. 2007, galx prop, 91

Shivvers, I., Filippenko, A. V., Silverman, J. M., et al. 2019, MNRAS, 482,1545

Tabatabaei, F. S., Schinnerer, E., Krause, M., et al. 2017, ApJ, 836, 185

Weingartner, J. C., \& Draine, B. T. 2001, ApJ, 548, 296

Westcott, J., Brinks, E., Hindson, L., Beswick, R., \& Heesen, V. 2018, MNRAS, 475,5116 